University of Rhode Island

DigitalCommons@URI

Open Access Dissertations

2011

\title{
MULTIVARIATE MULTISCALE ANALYSIS OF NATURAL AND ENGINEERED DYNAMICAL SYSTEMS
}

David B. Segala

University of Rhode Island, davidsegala@gmail.com

Follow this and additional works at: https://digitalcommons.uri.edu/oa_diss

\section{Recommended Citation}

Segala, David B., "MULTIVARIATE MULTISCALE ANALYSIS OF NATURAL AND ENGINEERED DYNAMICAL SYSTEMS" (2011). Open Access Dissertations. Paper 77.

https://digitalcommons.uri.edu/oa_diss/77

This Dissertation is brought to you for free and open access by DigitalCommons@URI. It has been accepted for inclusion in Open Access Dissertations by an authorized administrator of DigitalCommons@URI. For more information, please contact digitalcommons-group@uri.edu. 
MULTIVARIATE MULTISCALE ANALYSIS OF NATURAL AND ENGINEERED DYNAMICAL SYSTEMS

BY

DAVID B. SEGALA

A DISSERTATION SUBMITTED IN PARTIAL FULFILLMENT OF THE REQUIREMENTS FOR THE DEGREE OF DOCTOR OF PHILOSOPHY

IN

DEPARTMENT OF MECHANICAL, INDUSTRIAL, AND SYSTEMS ENGINEERING

UNIVERSITY OF RHODE ISLAND

2011 
DOCTOR OF PHILOSOPHY DISSERTATION

$\mathrm{OF}$

DAVID B. SEGALA

\section{APPROVED:}

Dissertation Committee:

$\begin{array}{ll}\text { Major Professor } \frac{\text { David Chelidze }}{} & \frac{\text { Gerhard Muller }}{\text { Philip Datseris }} \\ & \frac{\text { Nasser H. Zawia }}{\text { DEAN OF THE GRADUATE SCHOOL }}\end{array}$

\section{UNIVERSITY OF RHODE ISLAND}

2011 


\begin{abstract}
Most natural and engineered systems possess both spatial and temporal multiscale characteristics. In some complex systems (e.g., molecular dynamics and muscle fatigue dynamics), fast-time scales may dominate and/or obscure slow-time processes. In many practical situations, fast-time data is used to infer slow-time dynamics. The objective of this dissertation is to investigate the application of the newly developed multivariate analysis method of smooth orthogonal decomposition (SOD) to a general class of hierarchical dynamical systems. In particular, the SOD methodology was applied to a muscle fatigue accumulation study and protein dynamics.

The concept of phase space warping (PSW) refers to the deformations of fasttime phase space trajectories due to slow-time parameter drift. By characterizing these deformations caused by the slow-time processes from only the fast-time dynamics, feature vectors can be developed which contain the slow-time information. The idea of SOD is to extract smooth deterministic trends from multivariate data by considering the temporal and spatial characteristics of the data set. By doing so, SOD identifies time functions (SOCs) that are smoothest in time and have maximal variance. Therefore, it is our hope that the slow-time dynamics captured in the PSW feature space is in a one-to-one relationship with the smooth deterministic trends identified by SOD.

We discuss several advances to the already existing technology of PSW and SOD which have led to considerable improvements in the results. The three main modifications to the PSW/SOD methodology are: (1) a new weighting function is used for the PSW feature estimation, (2) SOD is applied to the nonlinear (i.e., polynomial) expansion of the original SOD coordinates, and (3) ad-hoc F test is used to statistically determine the number of SOD coordinates needed to recon-
\end{abstract}


struct muscle fatigue.

For the muscle fatigue accumulation, we hypothesize that: (1) SOD analysis of measured fast-time motion kinematics can be used to reconstruct the slow-time dynamics of muscle fatigue to establish a mapping between the kinematics and fatigue, and (2) a nonlinear extension of SOD can identify more optimal fatigue coordinates to provide a lower-dimensional reconstruction of the fatigue dynamics. These hypotheses were tested using movement kinematics recorded in ten subjects performing a high and low sawing motion and three load carrying walking Army soldiers. Independent local and global fatigue markers indicated by traditional surface electromyography (EMG) (recording of the electrical signals generated by individual muscles during a task) and breath-by-breath oxygen consumption $\dot{V} O_{2}$, respectively, were used for validation.

SOD analysis indicates that the linear SOCs are contaminated with more high-frequency content than the corresponding nonlinear coordinates for the same modal index across all subjects and experiments. This resulted in considerably fewer nonlinear SOCs needed to reconstruct the muscle fatigue trend compared to the linear SOCs. Between subject variability $R^{2}$ values, which are used to determine the quality of fits, indicate roughly a 20 dimensional subspace is needed to reconstruct the EMG trends using linear SOD analysis. However, utilizing nonlinear SOD, it was shown that in certain cases, where fatigue was actually present, a three to five dimensional subspace of cubic SOCs was sufficient. Similar results were seen in traditional $\dot{V} O_{2}$ trends. Thus, nonlinear SOD extracts more optimal coordinates than linear SOD, and in doing so, reduces the required dimensionality by roughly one order of magnitude. In addition, roughly 20 linear SOD modes were needed to capture a majority of the energy in the movement kinematics, which confers with the $R^{2}$ values. 
For the protein dynamics we hypothesize that: (1) SOD analysis of a full scale short time simulation can identify slow-time modes, and (2) slow coordinate subspaces will contain the peptide conformational geometry and information about the amount of time the peptide stays in each conformation. These were demonstrated by simulating a five residue cyclic pentapeptide-cyclo(d-Pro1-Ala2-Ala3Ala4-Ala5) which was previously assumed to oscillate between two conformational states. SOD analysis of Cartesian position and velocity components of the atoms in the backbone chain show some manifestation of rigid body modes. SOD analysis of transformed generalized internal coordinates extracts five dominant slow-time modes whose corresponding coordinates extract the conformational transitions of the peptide. Furthermore, the slow-time subspace identifies 33 conformational states instead of two and the total time spent in each conformation is found to range from $0.107 \%$ to $11.478 \%$ of the total time. 


\section{ACKNOWLEDGMENTS}

I am extremely grateful to many people who supported and helped me in the writing of this dissertation and throughout my time in graduate school.

First and foremost, I would like to thank my advisor, Dr. David Chelidze, who has helped me transform from an undergraduate student, to a graduate student, to a scientist. You have taught me how to pose a scientific problem, conduct effective research to answer the proposed hypothesis, and present my findings in a clear and concise manner. We have endured through good times and bad to produce the work I have here today. For this, I thank you.

I would like to thank my committee members: Dr. Philip Datseris, Dr. Gerhard Müller, Dr. David Taggart, and Dr. Leonard Kahn, for help with this dissertation as well. I have gained the knowledge to complete this work in all of your classes, and for that, I thank you all.

I am thankful to the nonlinear dynamics working group Ming Liu, Mike Falco, and Nguyen Hai Son for great conversations and support through my studies.

To my parents Jim and Barbara and brother Michael who have supported me throughout this whole endeavor. You have taught me perseverance which gave me the focus and ability to achieve this work. You have supported me in all of my decisions and for that, I thank you.

Last but certainly not least, to my wife Jen and my daughter Lindsey, who will be born in three months, you are both an inspiration to me. 


\section{DEDICATION}

This dissertation is dedicated in the loving memory of my grandfather Jerry Weiss and uncle Kevin Beardsworth. 


\section{TABLE OF CONTENTS}

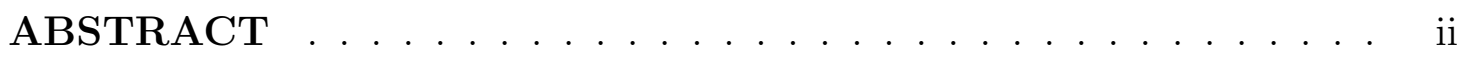

ACKNOWLEDGMENTS .................. . .

DEDICATION ...................... vi

TABLE OF CONTENTS $\ldots \ldots \ldots \ldots \ldots$ vii

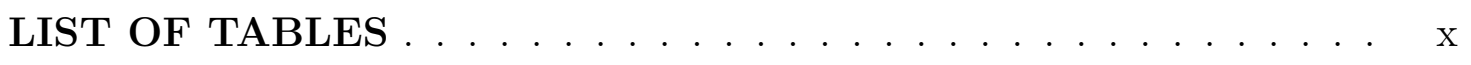

LIST OF FIGURES . . . . . . . . . . . . . . xi

\section{CHAPTER}

1 Introduction . . . . . . . . . . . . . . . . 1

1.1 Phase Space Warping . . . . . . . . . . . . . . . . . . . 3

1.2 Smooth Orthogonal Decomposition . . . . . . . . . . 6

1.3 Muscle Fatigue . . . . . . . . . . . . . . . . . . . . 8

1.4 Protein Dynamics . . . . . . . . . . . . . . . . . . . . . . . 14

2 Phase Space Warping . . . . . . . . . . . . . . . 19

2.1 PSW-A short-time tracking procedure . . . . . . . . . 22

2.2 PSW - A time series approach $\ldots \ldots \ldots \ldots \ldots$

2.2 .1 Phase space reconstruction . . . . . . . . . . . . . 23

2.2 .2 Tracking function . . . . . . . . . . . . . 27

2.2 .3 Vector valued feature space $\ldots \ldots \ldots \ldots . \ldots . \ldots 32$

2.2.4 Weighting function for the STRMP error . . . . . . . 35

3 Smooth Orthogonal Decomposition $\ldots \ldots \ldots \ldots$ 


\section{Page}

3.1 SOD-Continuous Formulation . . . . . . . . . . . . . . 42

3.2 SOD-Discrete Formulation . . . . . . . . . . . . . . . . . 47

3.3 Nonlinear SOD . . . . . . . . . . . . . . . . . . . 49

3.4 Properties of SOD . . . . . . . . . . . . . . . . 50

3.4.1 Invariance to invertible coordinate transforms . . . . . . 50

3.4.2 Orthogonality of projection modes and smooth coordinates 51

3.5 Applications of SOD . . . . . . . . . . . . . 53

3.5.1 Recovering harmonic signals contaminated with white noise 53

3.6 SOD Extracts Linear Normal Modes . . . . . . . . . . . . 56

3.6.1 Approximation of linear normal modes in a lumped massspring system . . . . . . . . . . . . . 56

3.6.2 Approximation of linear normal modes in a continuous beam . . . . . . . . . . . . . . . 59

4 Numerical and Experimental Protocols . . . . . . . . . . . . 64

4.1 Subjects Performing a Sawing Motion . . . . . . . . . . . . 64

4.1 Subjects .................... 64

4.1.2 Experimental Protocol . . . . . . . . . . . . 65

4.1.3 Data Analysis . . . . . . . . . . . . 67

4.2 Load Carrying U.S Army Soldiers . . . . . . . . . . . . . . . . . 74

4.2 .1 Subjects . . . . . . . . . . . . . . 74

4.2.2 Experimental Protocol . . . . . . . . . . . . 74

4.2 .3 Data Analysis . . . . . . . . . . . . . . . 76

4.3 Molecular Dynamics of Cyclic Pentapeptide . . . . . . . . . . 80

4.3.1 Molecular Simulation . . . . . . . . . . . . . 80 


\section{Page}

4.3 .2 OPLS Force Field . . . . . . . . . . . . . . 82

4.3.3 Data Preprocessing ............... 85

5 Results ........................ 87

5.1 Fatigue Tracking-Sawing Subjects . . . . . . . . . . . . 87

5.1.1 High Experiment . . . . . . . . . . . . . 87

5.1.2 Low Experiment . . . . . . . . . . . . . . 98

5.2 Fatigue Tracking-Army's Walking Subjects . . . . . . . . . . . 106

5.3 Protein Dynamics . . . . . . . . . . . . . . . 118

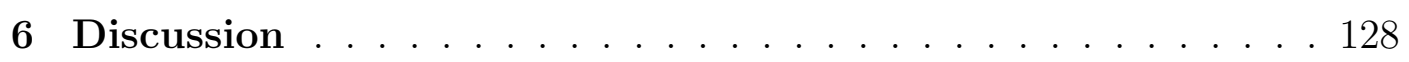

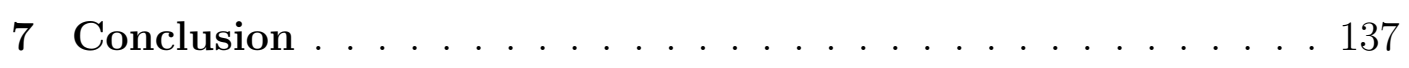

7.1 Future Work ....................... 139

LIST OF REFERENCES . . . . . . . . . . . . . . . . 141

\section{APPENDIX}

A List of Acronyms . . . . . . . . . . . . . . . . . . . . 149

B Sawing and Walking Subjects .............. 151

C SOCs Projections onto EMG Trends: Sawing Subjects . . . . 155

D SOCs Projections onto EMG Trends: Army Subjects . . . . . 173

BIBLIOGRAPHY . . . . . . . . . . . . . . . . . 177 


\section{LIST OF TABLES}

Table

Page

1 Basic characteristics for each sawing subject. . . . . . . . 65

2 Basic characteristics for each U.S Army soldier. . . . . . . . . . 74

3 Dihedral angles (in degrees) for Conformers A and B [1] . . . . 124

$4 \phi$ dihedral angles (in degrees) for all 33 conformation states . . 125

$5 \quad \psi$ dihedral angles (in degrees) for all 33 conformation states . . 126

$6 \quad \omega$ dihedral angles (in degrees) for all 33 conformation states . . 127

A.1 List of acronyms used throughout this dissertation . . . . . . 150

B.1 Basic characteristics for walking subject. For each subject and angle, 16 nearest neighbors were used in the local linear model and the reference phase space was slip into 32 hyper-boxes. . . . 152

B.2 Basic characteristics for each high sawing subject. For each subject and angle, 16 nearest neighbors were used in the local linear model and the reference phase space was slip into 32 hyper-boxes. 153

B.3 Basic characteristics for each low sawing subject. For each subject and angle, 16 nearest neighbors were used in the local linear model and the reference phase space was slip into 32 hyper-boxes. 154 


\section{LIST OF FIGURES}

Figure

Page

1 Illustration of the pathway by which a signal travels from the spinal cord to the motor unit into the neuromuscular junction.

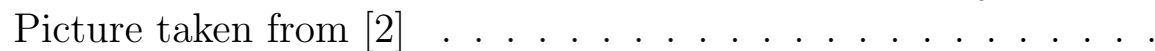

2 A skeletal muscle fiber is composed of numerous myofibrils that contain myofilaments of actin and myosin. Picture taken from [3] 9

3 An example recorded scalar time series over intermediate time intervals (Top plot). The reconstructed phase space of the recorded scalar time series using delay coordinate embedding. The delay time $\tau$ is found by the average mutual information (Bottom plot). . . . . . . . . . . . . . . 24

4 Example of a plot showing false nearest neighbors vs. embedding dimension. As the embedding dimension is increased the number of FNN is decreased until roughly $0 \%$. At this point, an embedding dimension of 5 would suffice. . . . . . . . . 26

5 Example of a plot showing average mutual information. We can clearly see the first local minimum to be around 18. This point is chosen as the delay coordinate. . . . . . . . . . . . 27

$6 \quad$ Graphical illustration depicting the construction of data records and reconstruction of the phase space in each data record. The time series is split into data records where the first data record reflects an unfatigued state. The phase space from each data record is reconstructed using time delay coordinate embedding.

7 A comparison showing the geometrical difference in the unfatigued (red) and fatigued (blue) reconstructed phase space trajectories. The unfatigued trajectory from the first data record and the fatigued trajectory is taken from the last data record. There is a clear distinction in the unfatigued and fatigued trajectories caused by the slow-time parameter drift. . . . . . . . 
8 Graphical illustration of the calculation of the tracking function $e_{R}$. At a snapshot in time, we see a portion of the current (black) and reference (red) trajectory. At a short $t_{s}$ apart, the point $y(i)$ drifted a small distance away from where it would have been in the reference trajectory. The tracking function is a measure of this deformation of the current point and its image in the reference phase space. . . . . . . . . . . . . .

9 (Left) A graphical illustration of the construction of a $k d$-tree for a $2 \mathrm{D}$ data set. The first cut (blue line) is along the median position of the $1^{\text {st }}$ coordinate. Each of these regions are partitioned at the median position along the $2^{\text {nd }}$ coordinate. Lastly, a partition is made along the $1^{\text {st }}$ coordinate again. (Right) The outline of the branch system corresponding to the cuts. . . . . .

10 Graphical illustration of the reference data record partitioned into disjoint hyper-boxes $\left\{\mathcal{B}_{i}\right\}$, in addition too, the reconstructed fast-time phase space trajectory. . . . . . . . . .

11 (Left) Graphical illustration of feature vector construction. Here, one feature vector is estimated from each $N_{r}$ data record. This projects the fast-time trajectory to a single point in the feature space. (Right) Graphical illustration of vector-valued feature space (multivariate time series). . . . . . . . . 35

12 2D example graphical illustration of how the estimated tracking function $e_{R}(y ; \phi)$ can align with either the short (left plot) or long (right plot) axis of an ellipse representing the cloud of nearest neighbors. There is much greater variability in the location along the axis of $e_{R}(y ; \phi)$ if the tip is along the long axis. . . . .

132 Draphical illustration of the weighting function. The cloud of nearest neighbors around point $y(i)$ will map to the cloud around $y(i+1)$ one time step later. However, because of the dynamical evolution of the system, the cloud of points will deform. Using the singular values $\left(\sigma_{1}\right.$ and $\left.\sigma_{2}\right)$ and the corresponding singular vector we can determine the shape and relative size of the cloud and its orientation with respect to the STRMP error, $e_{R}(y, \phi)$.

14 Mesh plots showing three different scenarios for possible values of the weighting function held constant. Left plot: $r_{s}=.5$, Middle plot: $\theta=\frac{\pi}{4}$, Right plot: $r_{\sigma}=.5 \ldots \ldots \ldots$ 
15 Graphical illustration of using SOD to extract a smooth deterministic trend (sine wave) which is embedded in a multivariate data set. . . . . . . . . . . . . . . . . . 41

16 (Left) A deterministic trend embedded int a (Right) high dimensional feature space. . . . . . . . . . . . . . .

17 (Left) The first three new basis coordinates found from applying SOD to the multivariate data set. The new basis is such that smooth coordinates have maximum variance and minimal local functions. (Right) Smooth coordinate extracted from the multivariate data set using SOD. . . . . . . . . .

18 (Top) Sinusoidal signal composed of harmonic frequencies, $x_{n}=$ $\sin (n \pi t)$ and (Bottom) extracted SOCs from ensemble matrix composed of signals. The extracted coordinates match considerably well with the signals. . . . . . . . . . . . . . .

19 (Top) Sinusoidal signal composed of harmonic frequencies, $x_{n}=$ $\sin (n \pi t)+\mathcal{N}(0,1)$ and (Bottom) extracted SOCs from ensemble matrix composed of signals. The coordinates match considerably well with the signals. There is some slight variation in the coordinates but the structure of the signal is partially recovered.

20 (Top) White noise $\mathcal{N}(0,1)$ and (Bottom) extracted SOCs from ensemble matrix composed of signals. In white noise, there is no signal to extract. . . . . . . . . . . . . . 55

21 Schematic of mass spring system. . . . . . . . . . . . 56

22 Time series from the simulation of the mass spring system, (Top) displacements $X_{1}$ and $X_{2}$, (Bottom) corresponding velocities $\dot{X}_{1}$ and $\dot{X}_{2} \ldots \ldots \ldots \ldots \ldots \ldots$

23 Calculated linear normal modes (- red) and smooth orthogonal modes (--blue) of the mass spring system. Except for the $4^{\text {th }}$ smooth mode, the modes approximate the LNM almost exactly.

24 Schematic diagram showing Moon's beam. The nonlinear form is realized by the two permeant magnets. The frame is driven by shacker at a certain frequency. . . . . . . . . . . . 59

25 Equilibrium position of beam. The equilibrium is found by setting $\ddot{X}=\dot{X}=0 \ldots \ldots \ldots$. . . . . . . . . . 62 
26 Calculated linear normal modes of the linearized system (-red) and smooth orthogonal modes (--blue) of the continuous beam. The smooth modes almost exactly extract the LNM. . . . . . . 63

$27 \quad$ Experimental setup for subjects sawing. Picture courtesy of Dr. Deanna Gates and Professor Jonathan Dingwell. . . . . . . . . . 66

28 Gross anatomy figure of the upper body showing where the muscles are located. . . . . . . . . . . . . . . . . . . . 68

29 Recorded raw Electromyography (EMG) time series for subject 6 for the high sawing experiment . . . . . . . . . . 69

30 Recorded raw Electromyography (EMG) time series for subject 6 for the low sawing experiment . . . . . . . . . . . 70

31 Mean power frequencies ( MNF ) calculated over individual cycles of EMG data of subject 6 for the high sawing experiment . 71

32 Mean power frequencies ( MNF ) calculated over individual cycles of EMG data of subject 6 for the low sawing experiment . . 72

33 Averaged MNF for subject 6 for the high sawing experiment . . 72

34 Averaged MNF for subject 6 for the low sawing experiment . . 73

35 Marker location for U.S Army soldiers. Picture courtesy of Dr. Jeffrey Schiffman . . . . . . . . . . . . . . 75

36 Experimental setup for U.S Army soldiers. Picture courtesy of Dr. Jeffrey Schiffman . . . . . . . . . . . . . . . 76

37 Gross anatomy figure of the lower body showing where the muscles are located. . . . . . . . . . . . . . . 77

38 Recorded EMG over the 120 minute march from each muscle for subject $10 . \ldots \ldots \ldots \ldots$

39 Calculated instantaneous mean frequency for each muscle and then averaged to a truncated time series of 63 points. . . . . . . 79 
40 (Left) Recorded $\dot{V} O_{2}$ over the 120 minute length of the experiment. Data greater then three standard deviations from the mean are removed. (Right) Averaged $\left\langle\dot{V} O_{2}\right\rangle$ over equal length windows after the convolution-based triangle filter is applied to the recorded data. . . . . . . . . . . . . . . . . . . . . . 79

41 Five residue cyclic pentapeptide-cyclo(d-Pro1-Ala2-Ala3-Ala4Ala5): (-blue) nitrogen atoms, (-red) oxygen atoms, (-green) carbon atoms. . . . . . . . . . . . . . . 81

42 (Left) Ramachandran plot after simulation that shows five different possible conformations of the peptide by the $\psi$ - $\psi$ dihedral angles. (Top Right) An rmsd plot of the peptide backbone after a least-squares fit to the initial backbone structure. (Bottom Right) Cosine of the angle between the C-O vector of Ala3 and the peptide ring plane defined by three atoms, Ala5 $\mathrm{C} \alpha$, Ala2 $\mathrm{C} \alpha$, and Ala3C'. Conformational transitions occur at 5.5, 11, and $13.7 \mathrm{~ns}$.

43 Schematic drawing showing the atomic configuration for bond stretching between two bonded atoms . . . . . . . . . . 83

44 Schematic drawing showing the atomic configuration for bending energy of a bonded triplet of atoms . . . . . . . . . . .

45 Schematic drawing showing the atomic configuration for nonbonded energy between non-bonded atoms $i$ and $j$. . . . . . 84

46 Schematic drawing showing the atomic configuration for torsion energy for each bonded quartet of atoms . . . . . . . . . .

47 Left: first 20 calculated SOVs for subject 6 humeral plain angle without the STRMP weighting function (blue o) and with the weighting function (red $*$ ). Right: first four corresponding dominant SOCs for subject 6 humeral plain angle without the STRMP weighting function (blue, - ) and with the weighting

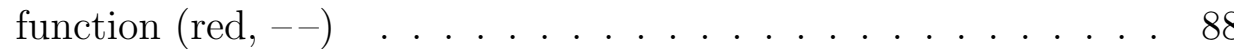


48 Plots showing the energy captured by POD (red $\circ$ ) and SOD (blue $*$ ) for subject 6 elbow flexion/extension (top), humeral plane angle (middle), and humeral elevation angle (bottom) for the high sawing experiment. The first column is the energy captured in each mode, the second column is the percent of total energy captured by each mode, and the last column is the energy captured in each subspace. . . . . . . . . . . . . . . . 90

49 Plots comparing calculated SOCs (red) and POCs (blue) for subject 6 elbow flexion/extension (top), humeral plane angle (middle), and humeral elevation angle (bottom) for the high sawing experiment. . . . . . . . . . . . . . . . . . . 94

50 Left plots: first 20 dominant linear (red ०), quadratic (blue *), and cubic (green $\diamond)$ SOVs for subject 6 elbow flexion/extension (top), humeral plane angle (middle), and humeral elevation angle (bottom) for the high sawing experiment. The corresponding first four dominant linear (red, thick -) , quadratic (blue, thin $--)$, and cubic (green, thin - ) (plots on the right). . . . . . . 95

51 Between subject variability $R^{2}$ values for MNF trends in the high sawing experiment for linear (red $\circ$ ), quadratic (blue $*$ ), and cubic (green $\diamond)$ SOCs. Error bars represent one standard deviation from the mean of $R^{2}$. Elbow flexion/extension (top), humeral plane (middle), and humeral elevation (bottom) angles. Values on top of each plot indicate the number of SOCs needed to adequately track EMG trends as determined from the F-test.

52 Linear combinations of linear (red --), quadratic (blue --), and cubic (green -) SOCs projections onto MNF (black -) markers in a least squares sense for subject 6 high sawing experiment: elbow flexion/extension (top), humeral plane (middle), and humeral elevation (bottom) angles. . . . . . . . . . .

53 Plots showing the energy captured by POD (red ०) and SOD (blue $*$ ) for subject 6 elbow flexion/extension (top), humeral plane angle (middle), and humeral elevation angle (bottom) for the low sawing experiment. The first column is the energy captured in each mode, the second column is the percent of total energy captured by each mode, and the last column is the energy captured in each subspace. . . . . . . . . . . . . . . . . . . 101 
54 Plots comparing calculated SOCs (red) and POCs (blue) for subject 6 elbow flexion/extension (top), humeral plane angle (middle), and humeral elevation angle (bottom) for the low sawing experiment. . . . . . . . . . . . . . . . 102

55 Left plots: first 20 dominant linear (red $\circ$ ), quadratic (blue $*$ ), and cubic (green $\diamond)$ SOVs for subject 6 elbow flexion/extension (top), humeral plane angle (middle), and humeral elevation angle (bottom) for the low sawing experiment. The corresponding first four dominant linear (red, thick -), quadratic (blue, thin $--)$, and cubic (green, thin -) (plots on the right). . . . . . 103

56 Between subject variability $R^{2}$ values for MNF trends in the low sawing experiment for linear (red $\circ$ ), quadratic (blue *), and cubic (green $\diamond)$ SOCs. Error bars represent one standard deviation from the mean of $R^{2}$. Elbow flexion/extension (top), humeral plane (middle), and humeral elevation (bottom) angles. Values on top of each plot indicate the number of SOCs needed to adequately track EMG trends as determined from the F-test. 104

57 Linear combinations of linear (red --), quadratic (blue --), and cubic (green -) SOCs projections onto MNF (black - ) markers in a least squares sense for subject 6 low sawing experiment: elbow flexion/extension (top), humeral plane (middle), and humeral elevation (bottom) angles. . . . . . . . . . . . 105

58 Plots showing the energy captured by POD (red ०) and SOD (blue $*$ ) for subject 10 lean (top), hip (middle top), ankle (middle bottom), and knee (bottom) angles in the army walking experiment. The first column is the energy captured in each mode, the second column is the percent of the total energy captured by each mode, and the last column is the energy captured in each subspace. . . . . . . . . . . . . . . . 110

59 Plots comparing calculated SOCs (red) and POCs (blue) for subject 10 lean (top), hip (middle top), ankle (middle bottom), and knee (bottom) angles in the army walking experiment. . . . 111 
60 Left plots: first 20 dominant linear (red o), quadratic (blue $*$ ), and cubic (green $\diamond$ ) SOVs for subject 10 lean (top), hip (middle top), ankle (middle bottom), and knee (bottom) angles in the army walking experiment. The corresponding first four dominant linear (red, thick - ), quadratic (blue, thin --), and cubic (green, thin -$)$ (plots on the right). . . . . . . . . . . 112

61 Between subject variability in $R^{2}$ values for IMNF trends for cubic SOCs. Error bars represent one standard deviation from the mean of $R^{2}$ for lean (top), hip (middle top), ankle (middle bottom), and knee (bottom) angles in the army walking experiment. Values on top of each plot indicate the number of SOCs needed to adequately track EMG trends as determined from the F-test. . . . . . . . . . . . . . . . . . 113

$62 R^{2}$ values for IMNF trends for cubic SOCs for subject 10 in the army walking experiment for lean angle (top), hip angle (middle top), ankle angle (middle bottom), and knee angle (bottom). Values on top of each plot indicate the number of SOCs needed to adequately track EMG trends as determined from the F-test. 114

63 Linear combinations of nonlinear SOCs (green, thin -) projected onto IMNF trends (black, thick --) in a least squares sense for subject 10: lean (top), hip (middle top), ankle (middle bottom), and knee (bottom) angles in the army walking experiment. . . . . . . . . . . . . . . . . . . . . 115

64 Estimated $\left\langle\dot{V} O_{2}\right\rangle$ trends for subject 7 (left), 8 (middle), and 10 (right) . . . . . . . . . . . . . . 116

65 Cross subject variability in $R^{2}$ values for estimated $\left\langle\dot{V} O_{2}\right\rangle$ trends for cubic SOCs. Error bars represent one standard deviation from the mean of $R^{2}$ for (D: lean angle), (B: hip angle), (A: ankle angle), and (C: knee angle). Values on top of each plot indicate the number of SOCs needed to adequately track $\left\langle\dot{V} \mathrm{O}_{2}\right\rangle$ trends as determined from the F-test. . . . . . . . . 116

66 Linear combinations of nonlinear SOCs (green, thin -) projected onto estimated $\left\langle\dot{V} O_{2}\right\rangle$ trends (blue, thick - ) in a least squares sense for subject 7 (top), 8 (middle), and 10 (bottom). . 117 
67 SOD analysis of cartesian coordinates from MD simulation. 40 dominant SOVs are depicted and the first three SOVs show some manifestation of rigid body modes. . . . . . . . . . . . . . . . 119

68 The first nine dominant SOCs are shown corresponding to the nine most dominant SOVs from the MD simulation. The SOCs are contaminated with high frequency content which could be a result of the rigid body motion. . . . . . . . . . . . . . . . 120

69 SOD analysis of generalized coordinates show four separated groups of SOVs corresponding to slow, faster, fast, and noise modes. . . . . . . . . . . . . . . . . 121

70 The first nine SOCs corresponding the nine most dominant SOVs. The first five dominant SOCs extract the flipping nature of the bond showing conformational changes. . . . . . . . 12

71 Faster coordinate subspace from SOD-based analysis of internal coordinates . . . . . . . . . . . . . . . 122

72 Fast coordinate subspace from SOD-based analysis of internal coordinates ...................... 12

73 Noise coordinate subspace from SOD-based analysis of internal coordinates . . . . . . . . . . . . . . . . 123

74 Slow coordinate subspace from SOD-based analysis of internal coordinates (Left) Two planes showing the two possible conformation states (Right) Rotation of the planes shows many discrete islands. . . . . . . . . . . . . . . . 123

C.1 Linear combinations of linear (red --), quadratic (blue --), and cubic (green -) SOCs projections onto MNF (black -) markers in a least squares sense for subject 1 high sawing experiment: elbow flexion/extension (top), humeral plane (middle), and humeral elevation (bottom) angles. . . . . . . . . . 156

C.2 Linear combinations of linear (red --), quadratic (blue --), and cubic (green -) SOCs projections onto MNF (black -) markers in a least squares sense for subject 1 low sawing experiment: elbow flexion/extension (top), humeral plane (middle), and humeral elevation (bottom) angles. . . . . . . . . . 157 
C.3 Linear combinations of linear (red --), quadratic (blue --), and cubic (green -) SOCs projections onto MNF (black -) markers in a least squares sense for subject 2 high sawing experiment: elbow flexion/extension (top), humeral plane (middle), and humeral elevation (bottom) angles. . . . . . . . . . 158

C.4 Linear combinations of linear (red --), quadratic (blue --), and cubic (green -) SOCs projections onto MNF (black - ) markers in a least squares sense for subject 2 low sawing experiment: elbow flexion/extension (top), humeral plane (bottom) angles. . 159

C.5 Linear combinations of linear (red --), quadratic (blue --), and cubic (green -) SOCs projections onto MNF (black -) markers in a least squares sense for subject 3 high sawing experiment: elbow flexion/extension (top), humeral plane (middle), and humeral elevation (bottom) angles. . . . . . . . . . . 160

C.6 Linear combinations of linear (red --), quadratic (blue --), and cubic (green -) SOCs projections onto MNF (black - ) markers in a least squares sense for subject 3 low sawing experiment: elbow flexion/extension (top), humeral plane (middle), and humeral elevation (bottom) angles. . . . . . . . . . . 161

C.7 Linear combinations of linear (red --), quadratic (blue --), and cubic (green -) SOCs projections onto MNF (black - ) markers in a least squares sense for subject 4 high sawing experiment: elbow flexion/extension (top), humeral plane (bottom) angles. . 162

C.8 Linear combinations of linear (red --), quadratic (blue --), and cubic (green -) SOCs projections onto MNF (black - ) markers in a least squares sense for subject 4 low sawing experiment: elbow flexion/extension (top), humeral plane (bottom) angles. . 163

C.9 Linear combinations of linear (red --), quadratic (blue --), and cubic (green -) SOCs projections onto MNF (black -) markers in a least squares sense for subject 5 high sawing experiment: elbow flexion/extension (top), humeral plane (middle), and humeral elevation (bottom) angles. . . . . . . . . . . 164 
C.10 Linear combinations of linear (red --), quadratic (blue --), and cubic (green -) SOCs projections onto MNF (black - ) markers in a least squares sense for subject 5 low sawing experiment: elbow flexion/extension (top), humeral plane (middle), and humeral elevation (bottom) angles. . . . . . . . . . . . . 165

C.11 Linear combinations of linear (red --), quadratic (blue --), and cubic (green -) SOCs projections onto MNF (black - ) markers in a least squares sense for subject 6 high sawing experiment: elbow flexion/extension (top), humeral plane (middle), and humeral elevation (bottom) angles. . . . . . . . . . 166

C.12 Linear combinations of linear (red --), quadratic (blue --), and cubic (green -) SOCs projections onto MNF (black -) markers in a least squares sense for subject 7 high sawing experiment: elbow flexion/extension (top), humeral plane (middle), and humeral elevation (bottom) angles. . . . . . . . . . . 167

C.13 Linear combinations of linear (red --), quadratic (blue --), and cubic (green -) SOCs projections onto MNF (black -) markers in a least squares sense for subject 7 low sawing experiment: elbow flexion/extension (top), humeral plane (middle), and humeral elevation (bottom) angles. . . . . . . . . . . . 168

C.14 Linear combinations of linear (red --), quadratic (blue --), and cubic (green -) SOCs projections onto MNF (black -) markers in a least squares sense for subject 8 high sawing experiment: elbow flexion/extension (top), humeral plane (middle), and humeral elevation (bottom) angles. . . . . . . . . . . . . 169

C.15 Linear combinations of linear (red --), quadratic (blue --), and cubic (green - ) SOCs projections onto MNF (black - ) markers in a least squares sense for subject 8 low sawing experiment: elbow flexion/extension (top) and humeral elevation (bottom) angles. . . . . . . . . . . . . . . . . . . . 170

C.16 Linear combinations of linear (red --), quadratic (blue --), and cubic (green -) SOCs projections onto MNF (black -) markers in a least squares sense for subject 9 high sawing experiment: elbow flexion/extension (top), humeral plane (middle), and humeral elevation (bottom) angles. . . . . . . . . . . . . 171 
C.17 Linear combinations of linear (red --), quadratic (blue --), and cubic (green -) SOCs projections onto MNF (black - ) markers in a least squares sense for subject 9 low sawing experiment: elbow flexion/extension (top), humeral plane (middle), and humeral elevation (bottom) angles. . . . . . . . . . . 172

D.1 Linear combinations of linear (red --), quadratic (blue --), and cubic (green - ) SOCs projections onto MNF (black - ) markers in a least squares sense for for subject 7: lean (top), hip (middle top), ankle (middle bottom), and knee (bottom) angles in the army walking experiment. . . . . . . . . . . . . . . . 174

D.2 Linear combinations of linear (red --), quadratic (blue --), and cubic (green -) SOCs projections onto MNF (black-) markers in a least squares sense for for subject 8: lean (top), hip (middle top), ankle (middle bottom), and knee (bottom) angles in the army walking experiment. . . . . . . . . . . . . . 175

D.3 Linear combinations of linear (red --), quadratic (blue --), and cubic (green - ) SOCs projections onto MNF (black - ) markers in a least squares sense for for subject 10: lean (top), hip (middle top), ankle (middle bottom), and knee (bottom) angles in the army walking experiment. ............ 176 


\section{CHAPTER 1}

\section{Introduction}

Multivariate multiscale characteristics are intrinsic properties in most natural and engineered dynamical systems. In these complex dynamical systems with dynamics on different time scales, fast-time scales may dominate and/or obscure slow-time processes. This may have a limiting factor in studying certain dynamical properties in particular spatial (i.e., statistical) and/or temporal (i.e., dynamical) scales. In many practical situations, fast-time data is used to infer slow-time dynamics, which is usually achieved by considering the spatial characteristics of the fast-time data.

The focus of this dissertation is on a newly developed multivariate multiscale data analysis method of smooth orthogonal decomposition (SOD), which considers

both spatial and temporal characteristics of the dynamical system. SOD is able to extract time functions from fast-time data that are smoothest in time and have maximum variance. It is hypothesized that this technique will find the smoothest low dimensional approximation of a high-dimensional dynamical model. In doing so, SOD can identify hidden slow deterministic processes obscured by faster and/or larger dynamics. This dissertation considers problems that are described by a general class of hierarchical dynamical systems where slow and fast-time dynamics are coupled through the parameters of the corresponding slow and fast-time subsystems. The SOD methodology will be applied to two different applications: (1) biomechanical motion kinematics will be used to identify physiologic muscle fatigue processes in humans, and (2) a short-time simulation of a peptide using a full scale molecular dynamics (MD) model will be used to identify slow-time dynamic modes and possible conformational states. 
Tracking the onset of physiologic fatigue is a scientific problem that has broad applications in both military and civilian activities. Providing countermeasures to fatigue in real-time settings is of considerable importance to soldiers and athletes. Deriving first principle fatigue accumulation models is an almost impossible task due to the complexities in creating a coupled model that includes physiological, neurological, and anatomical systems. However, there are various markers of physiological fatigue, based on oxygen consumption $\left(\dot{V} \mathrm{O}_{2}\right)$ (the capacity of a person to transport and utilize oxygen during incremental exercise), onset of blood lactate, and electromyography (EMG) (the measure of local electrical activity of the muscles). The procedure to obtain these markers can be invasive and may not be considered suitable to use in the field.

Clearly there is a need for the development of a physiologic fatigue tracking methodology that would utilize easily measurable variables for tracking the onset of muscle fatigue. It is hypothesized that (1) SOD analysis of measured fasttime motion kinematics can be used to reconstruct the slow-time dynamics of muscle fatigue to establish a mapping between the kinematics and fatigue and (2) a nonlinear extension of SOD can identify more optimal fatigue coordinates to provide a lower-dimensional reconstruction of the fatigue dynamics.

Molecular dynamic integrators are limited to a time step of one femtosecond $\left(10^{-15} \mathrm{~s}\right)$ because of atomic bond oscillation. Atomic bond oscillation is on the fast time scale; whereas, processes like folding dynamics and the mapping between local minima on free energy landscapes are on slower time scales $\left(10^{-15}-10^{1} \mathrm{~s}\right)$. Certain protein structures fluctuate on different time scales with different amplitudes. One goal of MD is to simulate protein folding and/or determine the map between local minima to investigate conformational dynamics. From there, free energy landscapes can give the energy associated with each conformation the protein 
can adhere to.

Inevitably, simulating molecular machines with several thousand amino acids on long time scales remains a challenge. Therefore, it would be advantageous to run a short simulation for a few nanoseconds to identify appropriate time scale modes to simulate the long time dynamics. It is hypothesized that (1) SOD analysis of a full scale short time simulation can identify slow-time modes and (2) slow coordinate subspaces will contain the peptide conformational geometry and information about the amount of time the peptide stays in each conformation.

\subsection{Phase Space Warping}

Methods of tracking material fatigue have played a large role in the field of structural health monitoring where structural response is related to the underlying damage process [4]. The physics community has investigated nonstationary systems with drifting parameters. Güttler et al. [5] used time series analysis methods to investigate smooth parameter variations in the equations of motion of dynamical systems [5]. In particular, time series data were used to construct feature vectors by calculating some standard or common long-time statistical measures. The selection of particular statistical measures was arbitrary.

Physiologic fatigue accumulation is, in several ways, similar to these problems of mechanical or structural fatigue where nonstationarity/damage (fatigue) accumulates slowly over time in the system characterized by faster dynamics (motion). The methodology from [5] which included the use of long time statistical measures were adopted in [6] to develop feature vectors from biomechanical data to track local muscle fatigue in standard EMG recordings. The long time statistical measures used were mean, variance, kurtosis, correlation sum, power spectral density, and fractal dimension. The feature vectors were not able to capture the underlying fatigue dynamics as indicated by EMG. A possible reason is that in experiments 
with human subjects, the time series may be short as compared to mechanical experiments. Therefore, there is a significant decrease or shortage of available data. Statistical measures that are defined for infinite time may not be realizable in a muscle fatigue context with limited data.

In addition, this method may be sensitive to bifurcations in the system which would result in sudden jumps in the long-time measures. It was suggested that these jumps or discontinuities disappear in slightly noisy systems; however, further work $[7,8]$ shows that the bifurcations can still cause discontinuities for real dynamical systems. Verdes et al. [9, 10] investigated other methods for reconstructing drifting parameters but there was only one scalar parameter variation. In this work, a multidimensional parameter variation is expected so other avenues must be explored.

Chelidze et al. [7] developed a method of tracking a hidden damage process, such as fatigue evolution, in a hierarchical dynamical system. In these particular systems, fast-time dynamics are directly observable dynamics; whereas, the slowtime dynamics are not directly observable. The fatigue evolution (or slow-time process) causes slow parameter drifts in a subsystem describing the fast-time dynamics. Therefore, trajectories in the fast-time phase space will slightly warp due to the parameter drift. The concept of phase space warping (PSW) describes these deformations of the fast-time trajectories $[7,11,12,13,8,14,6]$.

PSW was first used to track material fatigue in electro-mechanical systems [7]. In this electro-mechanical system, a cantilever beam oscillated between two potential wells. A slowly discharging 9-volt battery powered an electromagnet for one of the potential wells. This slowly discharging battery simulated the damage or slow-time process. The other well was realized by a permanent magnet and the beam was driven by a shaker. Using strain gauges, the fast-time dynamics were 
recorded from the beam. Using only the strain gauge data, a PSW-based tracking metric was in a one-to-one relationship with the slowly discharging voltage trend.

In [11], a physics-based model was developed to describe the experimental work from above. It was shown that by using this model, PSW was shown to track the evolution of the hidden damage (battery discharge). This technology was then applied to a nonlinear vibrating beam with an initiated crack, and the beam was vibrated until complete fracture occurred $[14,12,8]$. Using fast-time data recorded from strain gauges, the extracted slow-time trend was in a one-to-one correspondence with a Paris-type crack growth law used in the paper. The Paris-type crack growth law is a widely used damage model which describes the macroscopic evolution of crack growth. The equation can be written using a simple power law which relates the rate of crack growth per increasing load cycles, $d a / d N=c(\Delta k)^{m}$, where $N$ is the cycle count, $c$ is a small constant, $a$ is the crack length, $m$ depends on the material, and $\Delta k$ is the range of the stress intensity factor.

It was later hypothesized that since physiological fatigue is closely related to mechanical fatigue, the concept of PSW could be applied to biomechanical data to extract muscle fatigue dynamics. In one study, Dingwell et al. [15] had subjects walk on a treadmill as the inclination angle of the treadmill was slowly increased over time. The slowly increasing treadmill angle represented the slow-time damage process. Fast-time movement kinematics were recorded from the lower extremity of each subject. Using the movement kinematics, PSW was able to track the inclination angle of the treadmill.

This technology was also applied to highly trained stationary cyclists to see if PSW could track inherent fatigue variables in the body. It was found that recorded fast-time movement kinematics of the lower extremities contained the slow-time fatigue related information from standard Electromyography (EMG) analysis. 


\subsection{Smooth Orthogonal Decomposition}

In the past, conventional multivariate analysis techniques were achieved by Proper Orthogonal Decomposition (POD) [16, 17, 18, 19], Independent Component Analysis [20, 21], Linear/Nonlinear Normal Modes (LNMs/NNMs) [22, 23, 24, 25], etc. POD is also known as Principle Component Analysis [16, 26, 27, 28, 29], Singular Value Decomposition [16], and Karhunen-Loe've Decomposition [16, 30].

The purpose of POD is to reduce the dimensionality of a high dimensional system. Using POD, we can optimally approximate the lower dimensional dynamics of a high dimensional system. This is achieved under the constraint that the lower dimensional approximation retains as much variance as possible in the least squares sense of the high dimensional system. Provided an ensemble matrix whose columns contain mean subtracted time histories, POD extracts proper orthogonal modes (POMs - eigenvectors) or orthonormal basis vectors and proper orthogonal values (POVs-eigenvalues).

Proper orthogonal coordinates (POCs) are obtained by projecting the ensemble matrix onto a set of POMs. The POVs are arranged in decreasing order of energy so the first POM contains the most variance. The sum of all the POVs is equal to the total energy in the system. Feeny et al. [19] demonstrated that in a symmetric undamped free discrete vibration system with a uniform mass matrix, the POMs converge to the LNMs. This was also concluded for a continuous case [31]. However, in order to extract LNMs with POD, one must know the system's general mass matrix a priori. In addition, POD can not uniquely identify modal subspaces that have similar POVs [32].

Smooth orthogonal decomposition (SOD) is a multivariate multiscale data analysis method that can be thought of as an extension of POD [32, 6, 14, 12, $8,13,20,33,15]$. In addition to considering spatial (i.e., statistical) characteristics 
of the data set as in POD, SOD considers temporal (i.e., dynamical) characteristics of the data set as well. In particular, SOD identifies coordinates (smooth orthogonal coordinates - SOCs) that have both minimal temporal roughness and maximal spatial variance. Therefore, SOD obtains the smoothest low dimensional approximation of a high dimensional system.

Denoting the ensemble matrix by $Y$ and its time derivative by $D Y$, where $D$ is a discrete differential operator, then SOD is most often obtained by solving a generalized singular value problem of the matrix pair $Y$ and $D Y$. The generalized eigenvalues - squared singular values - are smooth orthogonal values (SOVs) and generalized eigenvectors are smooth projection modes (SPMs). As in the same way of obtaining POCs, SOCs are obtained by projecting $Y$ onto a set of SPMs. The degree of smoothness of the SOCs are described by the magnitude of the corresponding SOVs. Thus, the greater in magnitude the SOV, the smoother in time is the corresponding coordinate.

In [32], SOD was shown to produce comparable results to POD in estimating modal parameters in undamped, lightly damped, and distributed-parameter vibration cases. Since SOD is invariant to linear transformations, SOD-based modal identification does not require the mass matrix a priori. In contrast to POD, it was shown that SOD can separate signals according to their characteristic frequency components. If the signal is composed of lower frequency components, the SOCs will be smoother in time. Likewise, if the signal is composed of higher frequency components, the SOCs will be more jagged.

Chelidze and Liu [12] originally applied SOD to multivariate PSW features to extract deterministic (smooth) trends from a noisy PSW feature space [17]. Whether the feature space is obtained from recorded strain gauges or recorded biomechanical movement kinematics, SOD is able to extract smooth deterministic 
trends or identify time functions that are as smooth in time as possible. For the projects involving mechanical fatigue $[12,13,8,14]$, the dominant smooth trend extracted from the data is in a one-to-one relationship with the particular slow-time process (e.g., battery discharge or crack growth).

In the biomechanical field [15], SOD was applied to PSW-based feature space composed of recorded lower extremity movement kinematics of subjects walking on a treadmill. It was found that the increasing angle of the treadmill (i.e., slowtime process) was embedded in SOC space. Further application of PSW and SOD methodology to stationary cyclists showed that fatigue information present in the standard EMG analysis was also fully represented in the SOD coordinates extracted from the PSW features of cycling kinematics [6].

\subsection{Muscle Fatigue}

Muscle fatigue is defined as a decrease in the force generating capacity of a muscle or muscle group after activity $[34,35,36]$. In this dissertation, both local and global fatigue is of interest. Local fatigue will refer to the fatigue accumulation in each specific muscle (group). Global fatigue will emphasize whole body systemic fatigue. Global fatigue in the body is expected to accumulate monotonically assuming there is no influx of new external energy (e.g., food or energy drinks) into the system.

As a prelude to describing the physiological fatigue markers (EMG and $\dot{V} O_{2}$ ) and how they are used in this dissertation, the physiological processes used will be described for completeness. Local muscle physiology will be addressed to demonstrate the processes involved in muscle contractions followed by a discussion of global fatigue. The three main components that allow a muscle to contract are action potential, motor units, and calcium $\left(\mathrm{Ca}^{2+}\right)$. First, an electric impulse originates in the central nervous system. The signal propagates down the somatic 


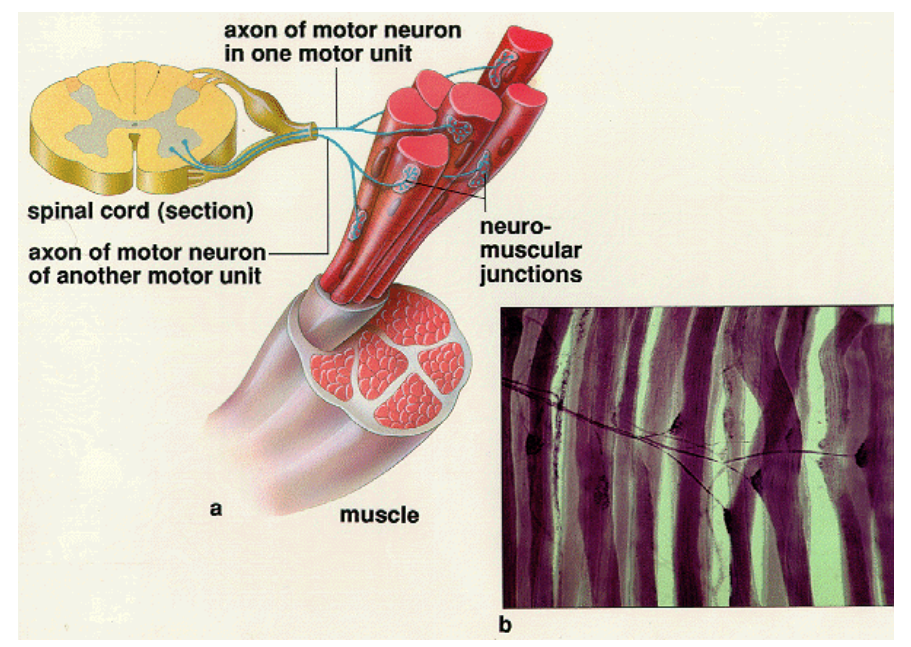

Figure 1. Illustration of the pathway by which a signal travels from the spinal cord to the motor unit into the neuromuscular junction. Picture taken from [2]

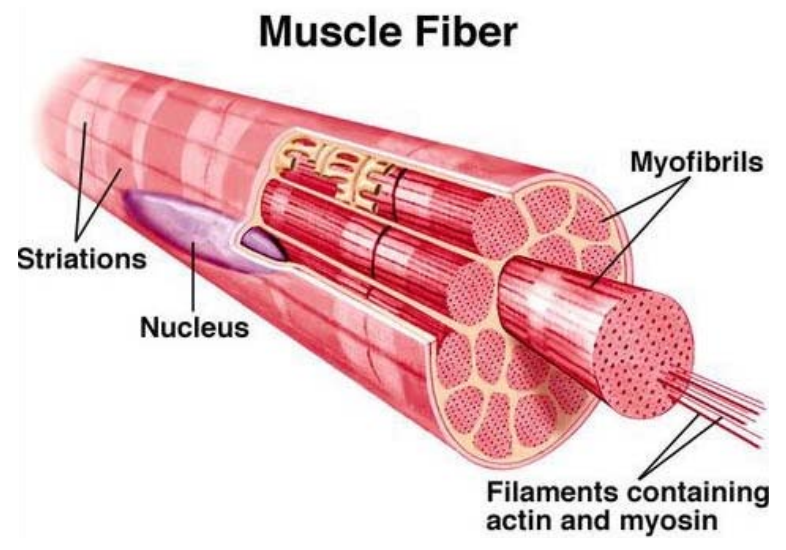

Figure 2. A skeletal muscle fiber is composed of numerous myofibrils that contain myofilaments of actin and myosin. Picture taken from [3]

motor neuron and causes a $C a^{2+}$ ion influx. The influx of $\mathrm{Ca}^{2+}$ causes a release of Acetylcholine (ACh) - a neurotransmitter chemical released by a neuron to communicate with other cells - into the motor end plate of the muscle fiber. This is the location of the neuromuscular junction (see Fig. 1). Each muscle fiber has one neuromuscular junction.

Due to the release of $\mathrm{ACh}$, the local transmembrane potential changes. This change results in the generation of the action potential that sweeps across the surface of the muscle fiber. The effect of the action potential depolarizes the inner 
portion of the muscle fiber (see Fig. 2). In doing so, the sarcoplasmic reticulum releases $\mathrm{Ca}^{2+}$ which binds to Troponin $\mathrm{C}$. This is the chemical present on the actin containing thin filaments of the myofibrils. This ends with a muscle contraction.

Allen et al. [37] gave several reasons for why muscles may fatigue: (1) the central nervous system may fail to produce action potentials, (2) the muscle action potentials may fail because of ionic changes, (3) energy stores can run out, (4) reactive oxygen species may damage proteins, and (5) structural damage to muscles. Fatigue during a sustained maximal contraction appears to be due to an accumulation of extracellular potassium (potassium leaves axons and muscle fibers during the repolarization phase). This reduces the membrane potential of muscle fibers and interferes with their ability to produce action potentials [38]. Also, a depletion of muscle glycogen and a reduced ability of the sarcoplasmic reticulum to release $\mathrm{Ca}^{2+}$ leads to a failure of excitation-contraction coupling [38].

Now, we focus on a more systemic global fatigue description. Skeletal muscles at rest obtain most of their energy from aerobic respiration of fatty acids. However, during exercise, muscle glycogen and blood glucose are also used as energy sources.

Glucose which is a simple sugar is broken down by glycolysis. The ultimate goal of glycolysis is the production of adenosine triphosphate (ATP), which is the major energy source in cellular respiration. In glycolysis, glucose is split into two pyruvic acid molecules and energy is released to form ATP. If there is enough oxygen, the pyruvic acid enters the mitochondria and produces more ATP. This is known as aerobic glycolysis due to the fact that oxygen is needed and there is a high yield of ATP.

If there is not enough oxygen, the pyruvic acid turns into lactic acid, which is known as anaerobic glycolysis. In addition, very little ATP is produced in the absence of oxygen. However, the lactic acid diffuses out of the muscle cell into the 
blood. This results in the burning and aching of muscles. At a certain point, the exercise has to be stopped, which results in more uptake of oxygen. Once there is enough oxygen, aerobic glycolysis starts again. It could be said that the lactic acid acts as the body's defense mechanism.

The lactate threshold is the point where the lactic acid builds up too much. This point is about $50-80 \%$ of the maximal oxygen uptake, or abbreviated $\dot{V} O_{2 \max }$. This value is determined by the person's height, weight, sex, age, etc. The average value in young males is $45 \mathrm{ml}$ per minute per kilogram [39]. $\dot{V} O_{2 \max }$ can be defined as the maximum capacity of an individual's body to transport and use oxygen during incremental exercise.

Based on the previous descriptions, first principle fatigue accumulation models are a very challenging problem due to the complexities of the human muscular and physiological systems. These human muscular and physiological systems are coupled events that span several time scales. For example, consider a person performing a repetitive task like sawing, walking, swimming, etc. At the start of this task, a person's gate or biomechanics (e.g., fast-time dynamics) should be reproducible over cyclic periods of motion. However, during this task, slow-time processes in the body (i.e., lactic acid production, motor unit recruitment, decrease in action potential velocities, etc.) lead to the onset of muscle fatigue. Once physiologic fatigue starts to accumulate, the body will compensate by altering its biomechanics to limit the use of the specific fatigued muscle(s) [40, 41, 42, 43, 44, 45]. Therefore, an alteration in the biomechanics, or fast-time dynamics, is a result of the fatigue accumulation, which is directly related to the slow-time processes.

Heavy load carriage and its effects on physical fatigue has not been fully investigated. To date, there have been no reported studies that examine the physiological or biomechanical effects of load carried continuously over a prolonged 
period of time. However, Frykman et al. observed gait data at the beginning and end of a period of carrying load [46]. It was observed here that there is a forward inclination of the trunk, reduced hip and knee angles at mid-stance, and decreased stride period and length.

Studies addressing the carrying of loads by humans have involved quantification of the effects that the weight of the external load has on energy consumption [47]. The metabolic costs of carrying loads for long bouts of walking were examined from oxygen uptake where subjects wore backpack loads [48, 49]. Results showed that given a backpack load weight and walking speed, a gradual increase in oxygen consumption was observed. Several mechanisms, such as a reduction in mechanical efficiency over time due to altered biomechanics, or simply the onset of fatigue within the skeletal muscles, were named as probable causes for the rise in energy cost with prolonged carrying of heavy loads.

Numerous studies have focused on methods of tracking and predicting muscle fatigue accumulation. A few fatigue tracking methods that require invasive procedures and/or cumbersome sensing can be found in $[50,51,52]$. Several physiological fatigue markers can be obtained from oxygen consumption $\left(\dot{V} O_{2}\right)$, onset of blood lactate and EMG [51, 52]. EMG has played a pivotal role as an indicator of local muscle fatigue and has been used in many tracking methodologies $[53,54,55,50,35,56]$.

The trend of the mean value of $\dot{V} O_{2}\left(\left\langle\dot{V} O_{2}\right\rangle\right)$ is assumed to carry global fatigue information. Unfortunately, there are several drawbacks in using $\left\langle\dot{V} O_{2}\right\rangle$ as an indicator of fatigue level. To obtain a stable measure of $\left\langle\dot{V} O_{2}\right\rangle$, one needs to average over a substantially large time window, which automatically limits its use in fast-paced tasks. In addition, as it was shown in [6], the individual can start a task at $\dot{V} O_{2}$ max load level. Therefore, at the beginning, muscles will have 
no fatigue and will fatigue gradually. However, $\dot{V} O_{2}$ will be at the saturation value $\left(\dot{V} O_{2 \max }\right)$ throughout the entire exercise. $\left\langle\dot{V} O_{2}\right\rangle$ is expected to be a good indicator of global or systemic fatigue only when a task starts below $\dot{V} O_{2}$ max load level and $\left\langle\dot{V} \mathrm{O}_{2}\right\rangle$ gradually approaches it.

The use of surface EMG has been explored extensively in trying to quantify changes in muscle function in fatigue [57]. Fatigue is a combination of both central and peripheral processes [36]. At the peripheral level, there is a loss of force generating capacity of individual motor units [58]. To maintain force, the central nervous system can increase its drive to the muscles. This causes already active motor units to fire more frequently and causes larger motor units to be recruited leading to an increased sense of effort [36]. As fatigue progresses, the number of active motor units decreases, muscle fiber conduction velocity decreases [59], motor units fire more slowly [34], and the motor units become more synchronized [60]. These changes lead to a decrease in the mean frequencies of the EMG signal [34] and eventually to task failure [61]. To this extent, mean frequencies have served as a good indicator of local muscle fatigue. Torvick et al. [50] examined several predictive measures (data mining, neural networks, and nearest-neighbors approaches) to try to track muscle fatigue through EMG. In addition, "short-time" Fourier transform methods have been developed [55] and subsequently validated [53, 54] that can track slowly varying changes in muscle fatigue across multiple dynamic contractions.

In all these cases, the primary fatigue marker used is not realizable in out-oflaboratory settings. Obtaining the marker data either requires cumbersome sensing equipment or invasive procedures. This dissertation focuses on a methodology that uses easily obtainable movement kinematics. This methodology has the robustness to track both local and global fatigue and can be applied in real-time and to 
variety of scenarios (e.g., subjects performing a sawing motion and load carrying U.S Soldiers).

\subsection{Protein Dynamics}

Classical molecular dynamic (MD) simulations have become a popular and powerful tool to describe and investigate molecular structure, dynamics, and functions of proteins since the first reported MD simulation in 1977 [62]. Structural fluctuations are often the key to the function of each particular peptide or protein. Essential information about this structure is found through nuclear magnetic resonance (NMR) and X-ray diffraction experiments. Dynamical processes cannot be characterized through the aforementioned experimental technologies. However, molecular modeling or MD simulations can provide not only dynamical information but structural information as well. Unfortunately, MD simulations are limited to a step size in the integration scheme which limit certain dynamical phenomenon to be studied.

Due to atomic bond oscillation molecular dynamic integrators are limited to a time step of one femtosecond $\left(10^{-15}\right)$. Atomic bond oscillation is on the fast time scale, whereas, processes like folding dynamics and the mapping between local minima on free energy landscapes are on slower time scales $\left(10^{-15}-10^{1} \mathrm{~s}\right)$. Certain protein structures fluctuate on different time scales with different ampli-

tudes. Local motions happen on the relatively short time scales $\left(0.01-5 \AA\right.$, $10^{-15}$ to $10^{-1} \mathrm{~s}$ ) while rigid body motions such as helix domain or subunit displacements involve larger amplitudes and significantly longer time scales $\left(1-10 \AA, 10^{-9}\right.$ to $10^{-1}$ s) [62]. Clearly, simulating molecular machines with several thousand amino acids on long time scales remains a challenge.

In [63], a new method of internal molecular dynamics was developed to simulate a molecule described by internal coordinates rather than conventional Carte- 
sian coordinates. The simulation space consists of bond angles, bond lengths, and torsion angles. It was suggested that using only these independent variables, it could be possible to eliminate all uninteresting fast degrees of freedom. In conjuncture with the work in [64], limiting integration time steps were investigated. It was found through internal coordinate MD simulations that the time steps currently used in MD simulations can be increased considerably with no serious loss in the accuracy of thermodynamic averages.

By nature, proteins try to find the conformation that allows them to be in their lowest energy state. Unfortunately, it is impossible to find the energy associated with each conformational state (e.g., geometrical configuration). For instance, even for a very small protein containing 10 atoms, the possible conformations are $\exp (10)$. Free energy landscapes are defined as a mapping of all possible conformations of a molecular entity, or the spatial positions of interacting molecules in a system and their corresponding energy levels, typically Gibbs free energy, on a twoor three-dimensional Cartesian coordinate system, [65]. Therefore, by knowing the free energy landscape, the conformational state can be determined that yields the lowest energy, which would be the conformational state to which the protein folds or misfolds.

To date, reduced order models have demonstrated significant results in describing dynamical phenomena such as the explorations of mappings between local minima on free energy landscapes, protein folding pathways, protein structure prediction, protein-protein association, and searches for ligand binding sites, etc., $[66,67,62,68,69,70,71]$. Reduced order models can be achieved by using a dimension reduction technique such as principle component analysis (PCA) to identify slow coordinate subspaces which are spanned by PCA modes (PC, principal components). Due to the fact that each degree of freedom has at least a 
nonlinear normal mode of vibration, proteins can contain hundreds to thousands of modes. It is suggested that this fluctuating information can be described with only a few slow modes [27]. Altis et. al [66] suggested that if we assume we have a time scale separation of the slow motion along the first few PCs and the fast motion along the remaining PCs, the first PCs may capture the free energy landscape. The reaction coordinates to describe the free energy landscape would then be the first slow PCs.

Recently, Lange [28] demonstrated that PCA can yield slow collective coordinates to use within a dimension reduction description of conformational motions. In order to obtain sufficient results, all hydrogen atoms were removed and 50 modes were needed to identify a suitable subspace. Lange [72] also found substantial evidence that indeed low-dimensional PCA subspaces extracted from short (5ns) MD simulations of T4 lysozyme and crambin describe more then $90 \%$ of the total atomic displacements observed in a (>200ns) simulation.

Skjaerven et al. [62] used a normal mode analysis to investigate slow dynamics, or large amplitude conformational changes. This methodology is based on the hypothesis that the vibrational normal modes exhibiting the lowest frequencies describe the largest movements and are the most functionally relevant. It is extremely important yet experimentally challenging to study transitions between two conformational states. Skjaerven showed that once the normal modes that contribute the most to the conformational difference have been identified, it is possible to use the modes to a make a 'movie' of the transition. The modes that contribute the most were found by taking the product between the open and closed conformations and the full set of normal modes. This was applied to Escherichia coli chaoeronin GRoEL-GroES ( 8000 amino acids) and I-domain of the $\alpha L \beta 2$ integrin ( 200 amino acids). Petrone et al. [67] performed a similar study to 
see how many normal modes are needed to map a conformation change. By projecting the conformational change onto a subspace spanned by the normal modes vectors, it was found that the first 20 modes only contribute $50 \%$ or less to the total conformation change. This was applied to several systems including myosin, calmodulin, NtrC, and hemoglobin. It was concluded that many more $(>700)$ modes are needed to accurately map the conformational change.

Maisuradze et al. [27] gave three limitations of PCA's ability to sort modes based on amplitudes: (1) the set of modes capturing the major fluctuations of a system depend on the width of the sampling time window. If the width of the sampling window increases, more and more slower modes can acquire larger amplitudes, (2) random diffusion can produce cosine-shaped principle components which can mistakenly be interpreted as the transition of the system from one state to another, and (3) the importance to eliminate overall rotation for large amplitude motion, on which PCA results largely depend on. This last limitation is overcome by the recent development of dihedral PCA (dPCA). In dPCA cartesian coordinates are transformed into more generalized internal coordinates or dihedral angles. Altis used dihedral angles instead of cartesian coordinates in PCA. It was shown that dPCA amounts to a one-to-one representation of the original angle distribution and its principal components can readily be characterized by corresponding conformational changes. In both [69] and [73] free energy landscapes were reconstructed using $\mathrm{dPCA}$ of a short time simulation. It was found that PCA finds only one free energy minimum; however by including internal coordinates dPCA finds numerous free energy minima, which corresponds to the true free energy landscape.

Maisuradze et al. also studied the folding dynamics of the triple $\beta$-strand WW domain from the Formin-binding protein 28 (1E0L in Protein Data Bank 
notation) and simulate the folding dynamics of small- and medium-sized proteins. Combining the fast-, slow-, and non-folding MD trajectories and calculating the first few dPCs, the free energy profiles and landscapes were constructed. Two global basins with local minima and the transition states were identified. It was concluded that the first one or two dPCs may contain the largest part of the total fluctuations of the system and the dimensions of the free energy landscapes can be reduced by one or two. Altis et al. [66] were able to construct a lowdimensional free energy landscape of the folding of hepta-alanine in explicit water using dPCA. It was shown that the calculated five-dimensional energy landscape was a suitable and accurate representation of the full-dimensional landscape by comparing metastable states of the full-dimensional system. Furthermore, this low-dimensional free energy landscape was used to study the dynamics of the lowdimensional system.

Based on the previous discussion, investigators have been using PCA to find slow modes. With the introduction of dihedral angles, the results have improved, however, there is much room for improvement. While PCA optimally finds the lowest dimensional approximation of a high dimensional system, it does not find the best slow modes to approximate the slow-time processes. However, it is the goal of SOD to extract the smoothest low dimensional approximation by extracting time functions that are smoothest in time. This dissertation focuses on the use of SOD to extract slow modes from a short-time simulation. Using shorttime simulations is extremely beneficial if simulation times are limited and if data storage is limited. In addition, these slow coordinate subspaces give information about the conformational geometry and the length of time the protein stays in each conformation. 


\section{CHAPTER 2 Phase Space Warping}

Deriving first principle muscle fatigue accumulation models is a very hard problem due to the complexities of the human muscular and physiological systems. One would have to first consider the physiological processes. Here, dynamic events such as motor unit recruitment, action potential velocities, lactic acid accumulation, etc., would have to be accounted for. These systems evolve on a slow-time scale when compared to movement kinematics which evolve on the fast-time scale. From an anatomical perspective, modeling of fast-time dynamical processes such as actin-myosin interaction, myofibrils, muscle fibers, and muscle-ligament interactions, which are also coupled events, would have to be considered. Lastly, the fast-time movement kinematics would have to be captured.

From a global standpoint, muscle fatigue accumulation is a coupled dynamical process that spans several time scales as briefly described above. For instance, as a person starts to perform a repetitive task (i.e., sawing, walking, swimming, etc.) their gate or biomechanics (e.g., fast-time dynamics) should be very reproducible over cyclic periods of motion. However, slow-time processes in the body (i.e., lactic acid production, motor unit recruitment, decrease in action potential velocities, etc.) start to change. In doing so, this change in the slow-time process will start to alter the biomechanics, or fast-time dynamics, very slightly. The changes in the biomechanics are so small at first that they may not be noticeable but after the onset of muscle fatigue the change in biomechanics can be drastic.

Instead of using first principle fatigue accumulation models, we can use the model that is presented in Eqs. (1a)-(1b). This model views muscle fatigue as evolving in a hierarchical dynamical system where slow-time fatigue and fast-time 
locomotion dynamics are coupled through parameters of a fast-time subsystem:

$$
\begin{gathered}
\dot{x}=f(x, \mu(\phi), t) \\
\dot{\phi}=\varepsilon g(\phi, x) \\
y=h(x) .
\end{gathered}
$$

where the variables in the equations carry a one-to-one relationship with processes in the human body. The variable $x \in \mathbb{R}^{n}$ is a fast-time dynamic vector variable describing movement kinematics which is directly observable. The variable $\phi \in \mathbb{R}^{m}$ is a slow-time dynamic vector variable describing $m$-dimensional muscle fatigue evolution which is not directly observable. $\phi$ can be considered a generalized coordinate of fatigue variables (e.g., some combination of motor unit recruitment, action potential velocities, lactic acid accumulation, etc). Also, $\phi$ alters a parameter vector $\mu$ in the fast-time system. As with many cases, $t$ is time and $\varepsilon$ is a small positive rate constant describing time scale separation between the fast-time and slow-time dynamics. A measurement function $h$ generating a scalar time series $y$ is based on the fast-time variable, $x$.

Even though we do not have access to the slow-time variable $\phi$ or the function $g(\phi, x)$, we usually do have direct access to the scalar time series, $y$. Therefore, in what follows we use the concept of phase space warping (PSW) to characterize the variations of the slow drift variable $\phi$ only from the fast-time dynamics over an intermediate window length. In essence, we use the scalar time series $y$ to reconstruct $\phi$ or more generally, the function $g(\phi, x)$.

In Eq. (1b), if $\varepsilon=0$, then $\dot{\phi}=0$ which makes the slow-time variable $\phi=$ constant. In this case, there is no fatigue. If $0<\varepsilon \ll 1$, then Eq. (1a) cannot be considered stationary because the parameters in $\mu$ are drifting, which would cause some invariant statistical measures to be functions of time. However, over 
some intermediate time length window $t_{r} \ll O\left(\varepsilon^{-1}\right)$, one could treat such a system as quasistationary. In this regard, $\phi$ can be considered constant over this 'short' time space, $t_{r}$, if over $t_{r}$, the variation in $\phi$ is negligible. The time series $y$ can be uniformly sampled at a sampling time $t_{s}$, where $t_{s} \ll t_{r}$.

Two main considerations have to be considered, (1) the sampling time $t_{s}$ and (2) the window length of $t_{r}$. Addressing the first concern of sampling time, if the sampling time of $t_{s}$ is too large, the fast-time dynamics may not be captured. On the opposite end, if the sampling time of $t_{s}$ is too short, data storage may become a limiting factor because of the abundance of data points. However, the sampling must be faster than the Nyquist criteria to adequately sample the fast-time dynamics. By the Nyquist criteria, the fast-time dynamics can be reconstructed from a time sequence of points as long as the sampling frequency is at least twice the highest frequency contained in the signal to avoid aliasing.

We can now consider the effect of the window length size. If the window length of $t_{r}$ is too large, then the variation of $\phi$ may not be negligible over this time span. If the window length of $t_{r}$ is too short, the fast-time subsystem may not be able to capture a statistically reliable representation of the fast-time phase space.

If we replace $\mu(\phi)$ by $\phi$ alone, our fast-time subsystem would be an explicit function of $\phi$. Since we view $\phi$ as a generalized fatigue coordinate then $\mu$ serves as a parameter mapping from the fatigue scale to the kinematic scale.

In what follows, the analytical formulation of phase space warping is presented. In an experimental context, access to the analytical solution is not available so the last section of the chapter will present this method if only a scalar time series is available. 


\subsection{PSW-A short-time tracking procedure}

The goal of PSW is to estimate the slow-time variable, $\phi$, using only the measured fast time variable $x$ in Eq. (1c). A general solution to Eq. (1a) can be written as $x(t)=X\left(t, t_{0}, x_{0}, \mu(\phi) ; \varepsilon\right)$. The initial conditions in the solution $x(t)$ are $\left(t_{0}, x_{0}, \phi\right)$ and we can define a prediction or observation time as, $\Delta t=t_{p}-t_{0}$. The prediction time is considered 'short' in the sense that $t_{s} \leq t_{p} \ll t_{r} \ll \frac{1}{\varepsilon}$. By requiring such a short prediction time, we expect the solutions to be smooth with respect to initial conditions and parameters. Furthermore, over this short-time interval we expect the slow variable $\phi$ to be approximately constant which allows us to treat the fast-time subsystem as quasistationary.

A reference model solution for $x(t)$ can also be written as $x(t)=$ $X\left(t, t_{0}, x_{0}, \mu\left(\phi_{R}\right) ; \varepsilon\right)$ where $\mu\left(\phi_{R}\right)$ represents the parameters for the reference fasttime system. Knowing these two solutions we can compare the fast-time dynamics at some current time $t_{0}$ to what the dynamics would have been, had it been in the reference state. We can write the short-time reference model prediction (STRMP) error as,

$$
e_{R}=X\left(t_{0}+t_{p}, t_{0}, x_{0}, \mu(\phi) ; \varepsilon\right)-X\left(t_{p}, t_{0}, x_{0}, \mu\left(\phi_{R}\right) ; \varepsilon\right)
$$

Taylor expanding the first term in Eq. (2) about $\phi=\phi_{R}$ leads to,

$$
X\left(t_{p}, t_{0}, x_{0}, \mu(\phi) ; \varepsilon\right)=X\left(t_{p}, t_{0}, x_{0}, \mu\left(\phi_{R}\right) ; \varepsilon\right)+\frac{\partial X}{\partial \mu} \frac{\partial \mu}{\partial \phi}\left(\phi-\phi_{R}\right)+O\left(\left\|\phi-\phi_{R}\right\|^{2}\right)
$$

Substituting Eq. (3) into Eq. (2) we have

$e_{R}=X\left(t_{p}, t_{0}, x_{0}, \mu\left(\phi_{R}\right) ; \varepsilon\right)+\frac{\partial X}{\partial \mu} \frac{\partial \mu}{\partial \phi}\left(\phi-\phi_{R}\right)+O\left(\left\|\phi-\phi_{R}\right\|^{2}\right)-X\left(t_{p}, t_{0}, x_{0}, \mu\left(\phi_{R}\right) ; \varepsilon\right)$

which reduces to

$$
e_{R}=\frac{\partial X}{\partial \mu} \frac{\partial \mu}{\partial \phi}\left(\phi-\phi_{R}\right)+O\left(\left\|\phi-\phi_{R}\right\|^{2}\right)
$$


which is to a leading order an affine transformation of the slow variable,

$$
e_{R} \approx C\left(t_{p}, t_{0}, x_{0}, \mu\left(\phi_{R}\right)\right) \phi+c\left(t_{p}, t_{0}, x_{0}, \mu\left(\phi_{R}\right)\right)
$$

where $C=\frac{\partial X}{\partial \mu} \frac{\partial \mu}{\partial \phi}$ is an $m \times n$ matrix and $c=-C \phi_{R}$ is an $m \times 1$ vector. The matrix $C$ can be considered the Jacobian and the vector $c$ is a bias. Since an affine transformation is in the form $y=a x$ one could always use a coordinate transformation to remove the bias term. In order for Eq. (6) to be a tracking function, matrix $C$ must have maximal rank. This condition is defined as a linear observability condition. If $C$ has maximal rank and $\varepsilon$ and $O\left(\left\|\phi-\phi_{R}\right\|^{2}\right)$ are sufficiently small, then the tracking function at any fixed point can be used to provide a linear measurement of, and therefore a means of tracking, the slow time variable $\phi$ using only the fast variable $x$. However, if $C$ does not have maximal rank, the linear condition fails but a nonlinear observability condition still may be possible. By using a nonlinear observability condition, we no longer have an affine transformation but we can still track the slow-time variable.

\subsection{PSW-A time series approach}

In an experimental context, we do not have access to the analytical solution, Eq. (6). However, an experimental scalar time series can be obtained for the fasttime variable $x$ like Eq. (1c). Since we only have access to a scalar time series, we can reconstruct the topologically equivalent phase space using the method of time delay coordinate embedding (phase space reconstruction) [74].

\subsubsection{Phase space reconstruction}

A scalar time series $\left\{y_{i}\right\}_{i=1}^{N}$ is recorded over intermediate time intervals, see Fig. 2.2.1. A $d$-dimensional state vector in the reconstructed phase space is described by,

$$
y(i)=\left[y_{i}, y_{i-\tau}, \ldots, y_{i-(d-1) \tau}\right]^{T}
$$



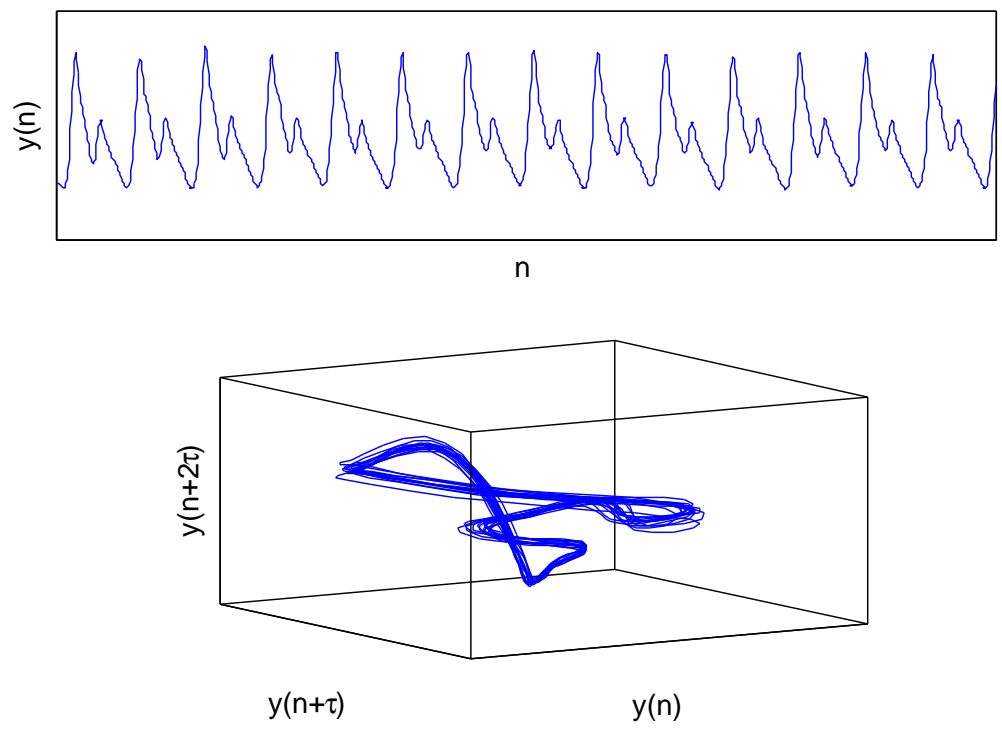

Figure 3. An example recorded scalar time series over intermediate time intervals (Top plot). The reconstructed phase space of the recorded scalar time series using delay coordinate embedding. The delay time $\tau$ is found by the average mutual information (Bottom plot).

where $T$ represents matrix transpose, $d$ is a sufficient embedding dimension, and $\tau$ is a delay time. Fig. 2.2.1 depicts the reconstructed phase space from the recorded scalar time series. The embedding dimension is usually estimated by the method of false nearest neighbors [75] and the time delay is usually estimated as the first local minimum of the average mutual information [76].

False nearest neighbors (FNN) is the method used to determine the appropriate embedding dimension of a trajectory in phase space by considering if two points next to each other are false nearest neighbors. The method is used to find the minimum number of time delay coordinates such that the trajectory does not cross itself. If two points are next to each other due to the dynamical evolution of the trajectory, then they are the nearest neighbors. If there is a point where the trajectory crosses itself in $d$-dimensional space, then that point or solution is not unique. Since solutions to ordinary differential equations must be unique, a 
higher dimension $(d+1, d+2, \cdots)$ is needed to completely unfold the trajectory such that there are no self-intersections. Takens' criteria guarantees embedding of a $D_{f}$-dimensional manifold $d>2 D_{f}$ dimensions [77].

In order to determine the embedding dimension of a trajectory we consider the trajectory first in a one-dimensional space. At this point, the FNN should be roughly around $100 \%$. Mostly, each neighbor is there because of the projection and not because of the dynamical evolution of the trajectory. Now, if we consider our trajectory in a two-dimensional space we will have eliminated some FNN because we have increased the dimension by one.

Several points that were neighbors in one-dimensional space may be on opposite sides of the two-dimensional phase space. Therefore, these points are in fact FNN. They were only neighbors because of the projection to a lower dimension and not because of the dynamics.

This process is iterated until the amount of FNN is less then some threshold value. At this point, the trajectory is considered unfolded with no self-intersections. It is not efficient to choose an embedding dimension larger than needed. By choosing too large an embedding dimension, you can increase the noise and you are increasing the number of time delay coordinates which can increase the computational time of some programs. By choosing too small an embedding dimension, the attractor is not completely unfolded and you will have FNN. It is important to note that if there is noise present, which is infinite dimensional, the amount of FNN will never reach zero. Fig. 4 depicts an example of the plot of FNN. The $x$-axis represents the embedding dimension with the associated FNN on the $y$-axis. As the embedding dimension is increased the amount of FNN decreases quite rapidly. For this example, an embedding dimension of 5 with some predetermined threshold would suffice. 


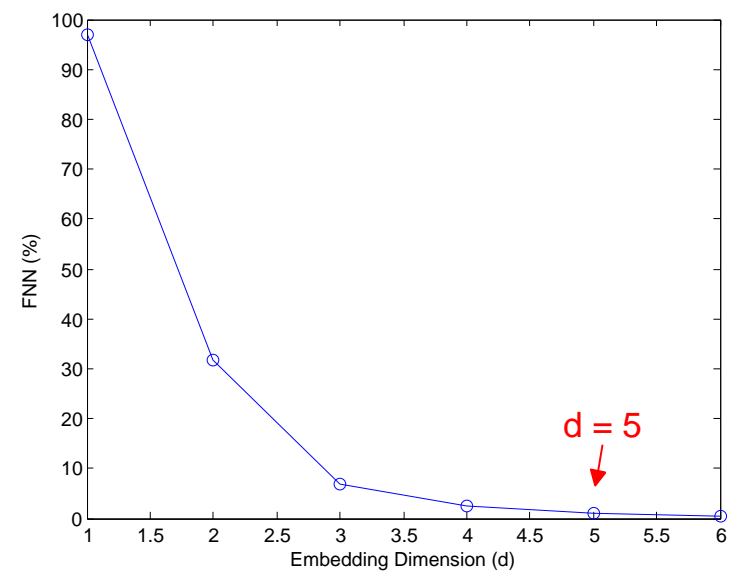

Figure 4. Example of a plot showing false nearest neighbors vs. embedding dimension. As the embedding dimension is increased the number of FNN is decreased until roughly $0 \%$. At this point, an embedding dimension of 5 would suffice.

When determining a value for the time delay it is noteworthy that there exists no rigorous way of obtaining an optimal value. It is also unclear what properties the optimal value should have [77]. Also, there is no real mathematical rigor as to what the value should be or how it should be estimated. The influence that the time delay has on the phase space reconstruction was investigated by Casdagli et al. [78]. However, in the paper no practical way of determining the value from a time series was alluded to. The first zero crossing of the autocorrelation integral has been used as an estimate of the time delay along with visual inspection. A problem with the autocorrelation function is that it is a linear statistic, so it does not account for any nonlinear characteristics [77]. However, the average mutual information does account for nonlinearities.

The method of average mutual information (AMI) - based on Shannon's entropy — was proposed originally in the field of information theory to give the mutual dependence of two random vectors, Fig. 5. The idea of AMI is to find a delay time $\tau$ such that mutual information, or redundancy is at minimum. AMI, $I(\tau)$, is a measure of how much information you know about $y(t+\tau)$ if you know 


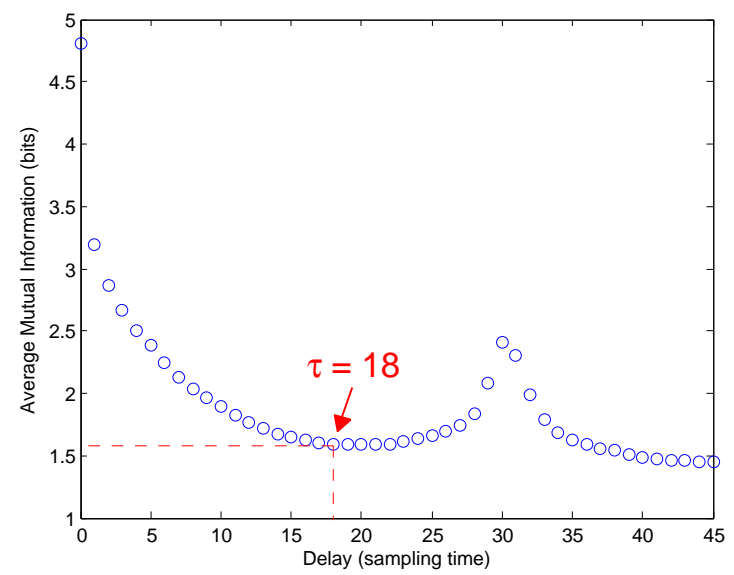

Figure 5. Example of a plot showing average mutual information. We can clearly see the first local minimum to be around 18. This point is chosen as the delay coordinate.

$y(t)$.

The first minimum of $I(\tau)$ marks the time lag where $y(t+\tau)$ adds the maximal amount of information to what we have from $y(t)$, or the time delay when the redundancy is the least. If $\tau$ is too small, then both $y(t)$ and $y(t+\tau)$ would basically be the same measurement or they would be correlated. Also, if $\tau$ is too large, then both $y(t)$ and $y(t+\tau)$ would basically be uncorrelated.

Mathematically, AMI is the sum of the two self-entropies minus the joint entropy and is expressed as

$$
I(\tau)=\sum_{i, j} p_{i j}(\tau) \ln p_{i j}(\tau)-2 \sum_{i} p_{i} \ln p_{i}
$$

where $p_{i}$ is the probability of the signal assuming a value inside the $i^{\text {th }}$ bin of a histogram of $y(t), p_{i j}(\tau)$ is the probability that $y(t)$ is in bin $i$ and $y(t+\tau)$ is in bin $j$.

\subsubsection{Tracking function}

The scalar time series $\left\{y_{i}\right\}_{i=1}^{N}$ is split into $N_{r}$ data records where the first data record is our reference data reflecting an unfatigued state, see Fig. 6 for 


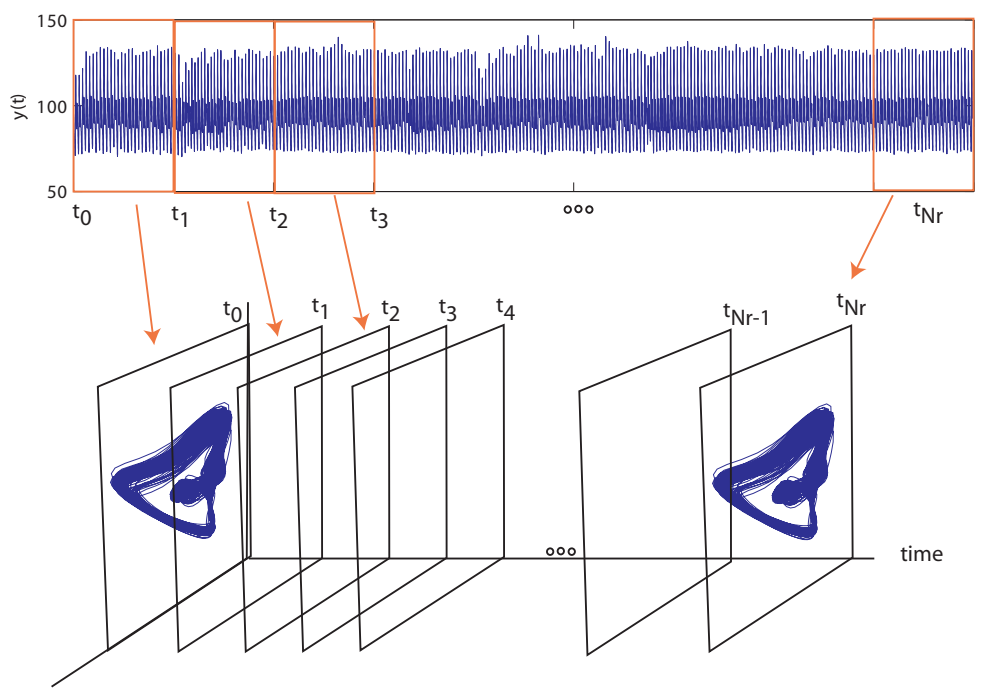

Figure 6. Graphical illustration depicting the construction of data records and reconstruction of the phase space in each data record. The time series is split into data records where the first data record reflects an unfatigued state. The phase space from each data record is reconstructed using time delay coordinate embedding.

illustration. Each data record contains approximately the same number of points. Once the time series is split into data records, the phase space of each data record is reconstructed. The same time delay and embedding dimension are used throughout each data record. Fig. 7 shows the evolution of the unfatigued (red line) and the fatigued (blue line) reconstructed phase space trajectories. The unfatigued trajectory is taken from the first data record and the fatigued trajectory is taken from the last data record. Clearly, there is a difference between the two trajectories, which is a result of the change in parameters caused by the slow-time dynamic variable drift.

It is assumed that the evolution of a point in the reconstructed phase space is governed by an unknown deterministic map, $P: \mathbb{R}^{d} \rightarrow \mathbb{R}^{d}$ which is usually nonlinear

$$
y(i+1)=P(y(i) ; \phi) .
$$




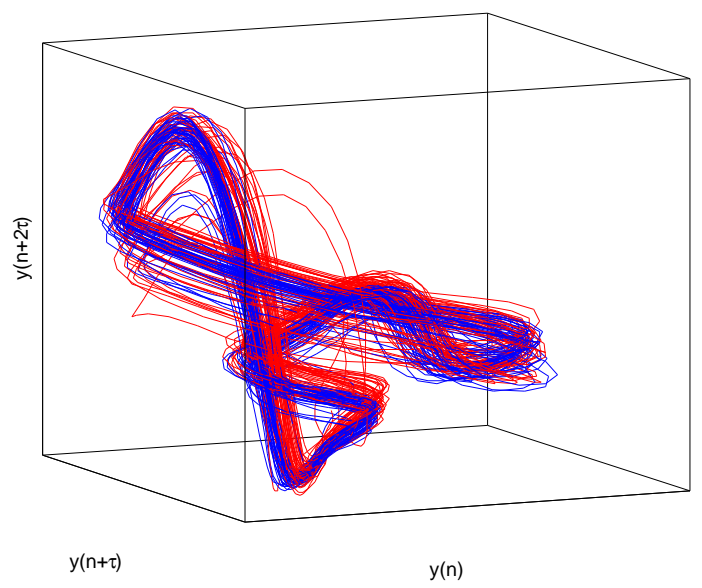

Figure 7. A comparison showing the geometrical difference in the unfatigued (red) and fatigued (blue) reconstructed phase space trajectories. The unfatigued trajectory from the first data record and the fatigued trajectory is taken from the last data record. There is a clear distinction in the unfatigued and fatigued trajectories caused by the slow-time parameter drift.

As in the analytical section we define the analog to Eq. (2), the PSW tracking function, that describes the change in short-time evolution of a trajectory for a point $y(i)$ in the reconstructed phase space as,

$$
e_{R}(y(i) ; \phi)=P(y(i) ; \phi)-P\left(y(i) ; \phi_{R}\right),
$$

where $t_{p}$ is equal to one time step, $\phi_{R}$ is the reference (unfatigued) state of the fatigue variable and $\phi$ is the current (fatigued) value. Eq. (10) is a substitute for the analytical solution in Eq. (3). This process can be seen graphically from Figs. 7 and 8. As we saw earlier, there is a clear difference in the two trajectories due to the slowly drifting fatigue variable. Fig. 8 depicts a graphical illustration of the unfatigued and fatigued trajectories for a single point. At a short $t_{s}$ apart, the point $y(i+1)$ drifted a small distance away from where it would have been in the reference trajectory. If there was no damage or fatiguing of the system, the point $y(i+1)$ would not have drifted away and would remain in the reference trajectory. This drift of the point and its image in the reconstructed phase space 


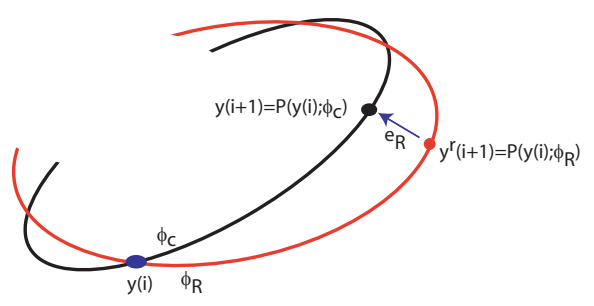

Figure 8. Graphical illustration of the calculation of the tracking function $e_{R}$. At a snapshot in time, we see a portion of the current (black) and reference (red) trajectory. At a short $t_{s}$ apart, the point $y(i)$ drifted a small distance away from where it would have been in the reference trajectory. The tracking function is a measure of this deformation of the current point and its image in the reference phase space.

is characterized by the tracking function.

Using Eq. (9) in Eq. (10) we can rewrite the tracking function as,

$$
e_{R}(y(i) ; \phi)=y(i+1)-P\left(y(i) ; \phi_{R}\right)
$$

since the value of $y(i+1)=P(y(i) ; \phi)$ is already known from the current reconstructed phase space. A local linear model can be used to approximate the value of the map $P\left(y(i) ; \phi_{R}\right)$ because there is usually no image of the point $y(i)$ in the reference reconstructed phase space. However, due to the smooth evolution of the system and the prediction time being short, we can approximate where the image would be in proximity to $N_{m}$ nearest neighbors. One drawback to not having exact image and access only to the nearest neighbors, is the error added to the local linear model. This error and a correction for it is discussed below. The parameters for the local linear model are determined in the least squares sense for each point $y(i)$ by finding its $\left\{y^{r}(i) \equiv y^{r}\left(i ; \phi_{R}\right)\right\}_{r=1}^{N_{m}}$ nearest neighbors and their images $\left\{y^{r}(i+1) \equiv y^{r}\left(i+1 ; \phi_{R}\right)\right\}_{r=1}^{N_{m}}$ one time-step later. We can express the local linear model for each point as

$$
P\left(y(i) ; \phi_{R}\right)=y(i+1)=A_{i} y(i)+b_{i},
$$

where $A_{i}$ is a model parameter matrix and $b_{i}$ is a parameter vector which are 
currently unknown. Solving for them we can rewrite the right hand side of Eq. (12) as,

$$
y(i+1)=A_{i} y(i)+b_{i}=B_{i} \hat{y}(i)
$$

In doing so, we concatenate $A_{i} \in \mathbb{R}^{d \times d}$ and $b_{i} \in \mathbb{R}^{d \times 1}$ into a matrix $B_{i} \in \mathbb{R}^{d \times(d+1)}$. Also, we concatenate $y(i)$ and a ONE to form the vector $\hat{y}(i)$. Finally we are left with,

$$
y(i+1)=B_{i} \hat{y}(i)
$$

Substituting $Y=\left[y^{1}(i), y^{2}(i), \cdots, y^{N_{m}}(i)\right]$ and $\hat{Y}=\left[\hat{y}^{1}(i), \hat{y}^{2}(i), \cdots, \hat{y}^{N_{m}}(i)\right]$ we can now solve for $B_{i}$ as,

$$
B_{i}=Y \hat{Y}^{T}\left[\hat{Y} \hat{Y}^{T}\right]^{-1}
$$

From here, $A_{i}$ is all but the last column of $B_{i}$ and $b_{i}$ is the last column of $B_{i}$.

We can now write the down PSW tracking function as,

$$
e_{R}(y(i) ; \phi)=\widehat{e}_{R}(y(i) ; \phi)+e_{M}(y(i))
$$

where $e_{M}$ is the local linear model error and $\widehat{e}_{R}$ is the estimated tracking function which is given by,

$$
\widehat{e}_{R}(y(i) ; \phi)=y(i+1)-A_{i} y(i)-b_{i}
$$

The use of $\widehat{e}_{R}(y(i) ; \phi)$ in place of $e_{R}(y(i) ; \phi)$ is allowable if $e_{M} \ll \widehat{e}_{R}(y(i) ; \phi)$. There are several main sources of fluctuations in the tracking function that are not related to the slow evolution of $\phi$ and are introduced through $e_{M}$. One source comes from the accuracy of the model from point-to-point in the reference phase space. If some regions of the phase space are more densely populated than others, there will be more nearest neighbors closer to the point of interest and will produce a more accurate model. Likewise, if some regions are sparser than others, the nearest neighbors may in fact be far away from the point of interest or there can just be 
fewer points. This may produce less accurate models. If the distribution of nearest neighbors around a point is thought of as a cloud, this cloud can change size and shape around the point of interest. Both of which can influence error in the local linear model.

Also, how the orientation of the cloud of nearest neighbors aligns with $e_{R}(y(i) ; \phi)$. If the cloud is oriented perpendicular to the tracking vector, more accurate models can be achieved. A final source of errors comes from experimental noise. To address the fluctuations, a weighted averaging scheme will be presented later.

\subsubsection{Vector valued feature space}

In experiments, trajectories will not traverse the entire phase space. Even in a chaotic system, where a large portion of the phase space is explored by trajectories. Therefore, areas of the reference phase space will have an unequal probability distributions of points. This can lead to inaccuracies in the local linear models that considers $N_{m}$ nearest neighbors of a particular point, $y(i)$. To compensate for this, the reference phase space is partitioned into $N_{e}$ disjoint hyper-boxes $\left\{\mathcal{B}_{i}\right\}_{i=1}^{N_{e}}$. Also, by partitioning we are able to cluster the same density and geometrical regions of the phase space.

Partitioning of the phase space can be achieved by one of two methods, either uniform partition or equiprobable partition. In a uniform partition, the phase space is divided into hyper-boxes where each box is the same size. In a equiprobable partition, the phase space is divided into hyper-boxes where each box has approximately the same amount of predetermined points and is the method that is used in this dissertation. Partitioning is achieved by using a kd-tree [79].

$k d$-tree is a binary tree and is used here for nearest neighbor searches of the point $y(i)$. The $k d$-tree algorithm partitions a data set about the median position 
of a coordinate axis of the data set to construct a hyper-box (a branch in the tree structure). As an end result, the phase space is partitioned into $N_{e}$ hyper-boxes. The points that are spatially close will share many branches in the $k d$-tree. These branches can then be searched for the nearest neighbors. The iterative process is carried out as follows.

For this iterative process example, consider the 2D space in Fig. 9. The data set in the reference phase space is first partitioned along the median position of the $1^{\text {st }}$ coordinate into two subset regions (blue, - ). This first partition forms a base for the $k d$-tree and is shown as the first node in Fig. 9 (Right). If the number of points in either subset is not equal to the predetermined number of points, that region is partitioned along the median position of the $2^{\text {nd }}$ coordinate into two subset regions. In this example, both subsets from the base (blue,-) are partitioned (red, $--)$ into two subsets. These new subsets form branches in the $k d$-tree. Now, in the $2 \mathrm{D}$ space, there are four subset regions. However, in the figure, region $\mathbf{A}$ does contain the predetermined number of points so no additional partition is needed for that particular region. But, each other subset needs to be partitioned along the $1^{\text {st }}$ coordinate again $($ green, $\cdots$ ). Finally, after three partitions each subset contains the predetermined number of points.

After the reference phase space is partitioned, Fig. 10, the weighted average STRMP error is estimated in each of these hyper-boxes:

$$
e_{i}(\phi)=\frac{\sum_{y \in \mathcal{B}_{i}} w(y)\left\|\widehat{e}_{R}(y ; \phi)\right\|}{\sum_{y \in \mathcal{B}_{i}} w(y)},
$$

where the weight $w(y)=w\left(y ; \phi_{R}\right)$ accounts for the accuracy of the reference local linear models used in the estimation. This is explained in more detail in the next section. For each data record $j\left(j=1, \ldots, N_{r}\right)$ these averaged errors are assembled into an $N_{e}$-dimensional feature vector,

$$
e^{j}=\left[e_{1}(\phi), e_{2}(\phi), \ldots, e_{N_{e}}(\phi)\right]
$$



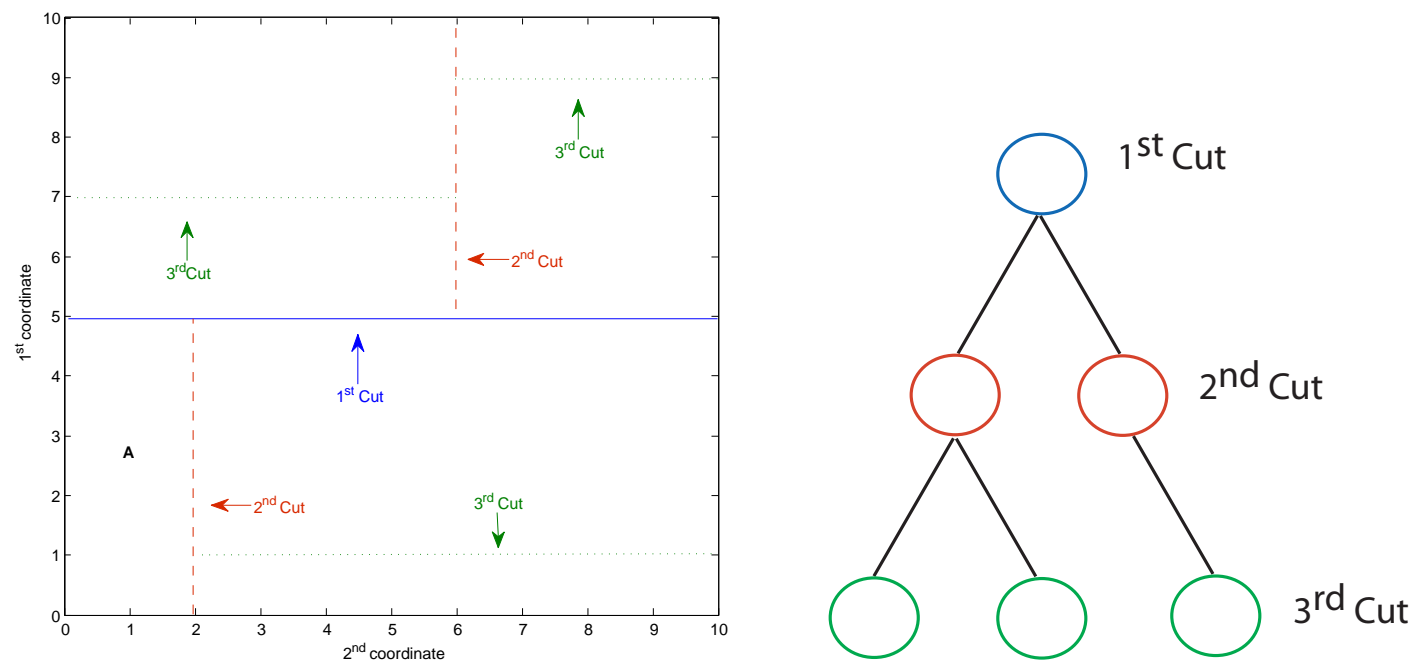

Figure 9. (Left) A graphical illustration of the construction of a $k d$-tree for a $2 \mathrm{D}$ data set. The first cut (blue line) is along the median position of the $1^{\text {st }}$ coordinate. Each of these regions are partitioned at the median position along the $2^{\text {nd }}$ coordinate. Lastly, a partition is made along the $1^{\text {st }}$ coordinate again. (Right) The outline of the branch system corresponding to the cuts.

The estimated feature vectors $e^{j}$ are then calculated for all $N_{r}$ data records and row-wise concatenated (in time sequence) into a vector-valued feature space (multivariate time series) where each row in $Y$ corresponds to a point in the feature space.

$$
Y=\left[e^{1} ; e^{2} ; \ldots ; e^{N_{r}}\right]
$$

Each column of $Y$ represents the time-series, but on the slow-time scale, of each component of the averaged tracking metric, $e_{i}(\phi)$ in each region, $\mathcal{B}_{i}$. The vale of $e^{j}$ in Eq. (17) maps the $j^{\text {th }}$ fast-time phase space data onto a single point in the feature space. Fig. 11 depicts both of these cases. In the left plot, each feature vector is shown in each $N_{r}$ data record. Furthermore, the right plot shows the entire vector-valued feature space, or the matrix $Y$. It is assumed that the slowtime fatigue information is embedded in this matrix. 


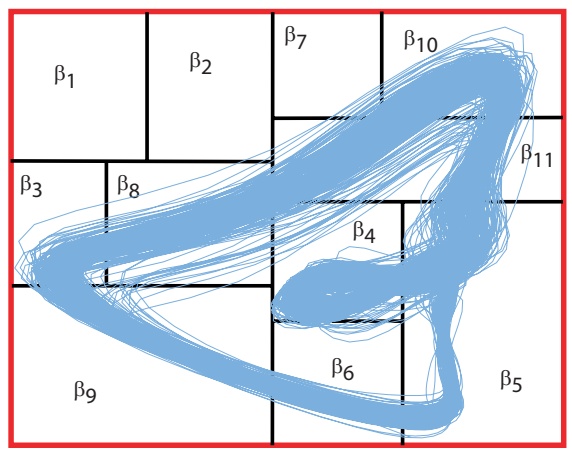

Figure 10. Graphical illustration of the reference data record partitioned into disjoint hyper-boxes $\left\{\mathcal{B}_{i}\right\}$, in addition too, the reconstructed fast-time phase space trajectory.
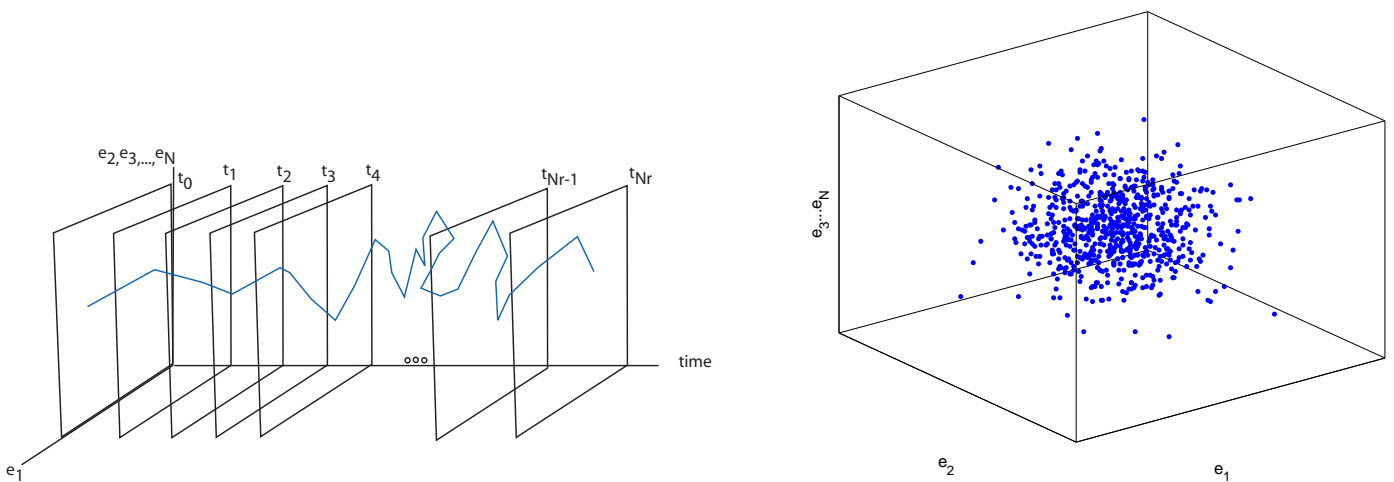

Figure 11. (Left) Graphical illustration of feature vector construction. Here, one feature vector is estimated from each $N_{r}$ data record. This projects the fast-time trajectory to a single point in the feature space. (Right) Graphical illustration of vector-valued feature space (multivariate time series).

\subsubsection{Weighting function for the STRMP error}

Due to the dynamical evolution of trajectories in the phase space of the system, points near the trajectories may vary significantly from one time step to another. As a result, two points in one data record may diverge or converge from each other in a data record one time step away. Looking at a larger picture, a point $y(i)$ surrounded by a cloud of nearest neighbors may not have the same nearest neighbors as $y(i+1)$. There could be new points that have migrated closer to the point or points could have migrated to a different spatial location in the phase 

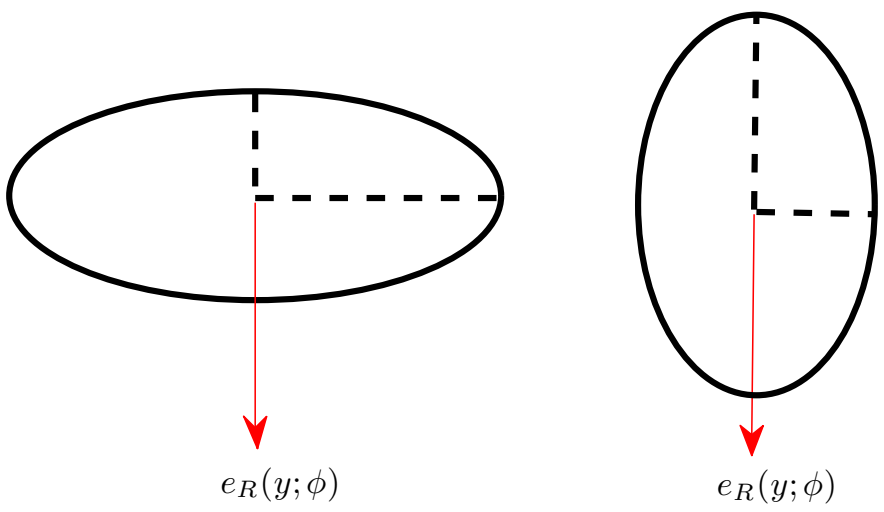

Figure 12. 2D example graphical illustration of how the estimated tracking function $e_{R}(y ; \phi)$ can align with either the short (left plot) or long (right plot) axis of an ellipse representing the cloud of nearest neighbors. There is much greater variability in the location along the axis of $e_{R}(y ; \phi)$ if the tip is along the long axis.

space resulting in regions with a denser or sparser amount of points. To this end, the local linear model about a point $y(i+1)$ will be a function of the probability density of points. Therefore, a cloud of nearest neighbors used for the local linear model will change shape and size from one point to the next.

Another concern with drifting points is how the cloud of nearest neighbors aligns with the estimated tracking function, $e_{R}(y ; \phi)$. For an example, consider the 2D cloud of nearest neighbors in Fig. 12. Each cloud is in an elliptical shape for illustration purposes. In the figure, $e_{R}(y ; \phi)$ can align with either the short (left plot) or long (right plot) axis of an ellipse representing the cloud of nearest neighbors. There is much greater variability in the location along the axis of $e_{R}(y ; \phi)$ if the tip is along the long axis.

In the previous two paragraphs, several reasons for why the estimated tracking function $\widehat{e}_{R}(y(i) ; \phi)$ in Eq. (15) may produce inaccurate estimates were presented. These reasons primarily dealt with the amount of nearest neighbors and the local linear model. Since the cloud of nearest neighbors does not stay constant for each point, we proposed the following weighting function.

In the weighting, we account for three factors contributing to the accuracy of 


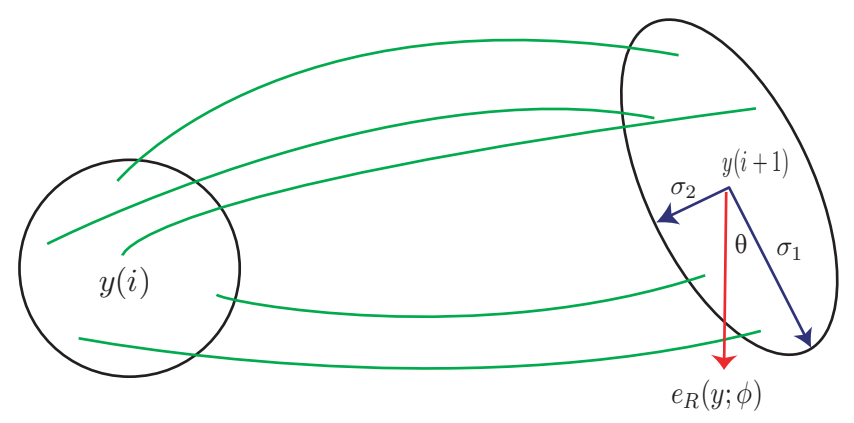

Figure 13. 2D graphical illustration of the weighting function. The cloud of nearest neighbors around point $y(i)$ will map to the cloud around $y(i+1)$ one time step later. However, because of the dynamical evolution of the system, the cloud of points will deform. Using the singular values $\left(\sigma_{1}\right.$ and $\left.\sigma_{2}\right)$ and the corresponding singular vector we can determine the shape and relative size of the cloud and its orientation with respect to the STRMP error, $e_{R}(y, \phi)$.

prediction: (1) the relative size of a cloud of reference nearest neighbors of $y(i+1)$, (2) the shape of the cloud, and (3) its orientation with respect to the STRMP error. These three factors are determined using singular value decomposition of the matrix containing all the nearest neighbor points of $y(i+1)$ (assumed to be mean subtracted). The graphical illustration is shown in Fig. 13. The resulting weighting function that accounts for each of the three listed factors is given for each point $y=y(i)$ as:

$$
w(y)=e^{-r_{s}(y)}[\sin \theta(y)]^{\left[1-r_{\sigma}^{2}(y)\right]},
$$

where,

$$
r_{\sigma}(y)=\frac{\sigma_{2}(y)}{\sigma_{1}(y)}, \quad r_{s}(y)=\frac{\sigma_{1}(y)}{\left\|\widehat{e}_{R}(y ; \phi)\right\|}, \quad \theta(y)=\measuredangle\left(s_{1}(y), \widehat{e}_{R}(y ; \phi)\right),
$$

and where $\sigma_{1}(y)$ and $\sigma_{2}(y)$ are the first two largest singular values of the corresponding cloud of reference points $y(i+1)$, and $s_{1}(y)$ is the singular vector corresponding to the largest singular value. Using Eq. (19) in Eq. (15), in addition to the size of a cloud $y(i+1)$, we account for both the degree of elongation of the cloud and the alignment of that elongation along the corresponding STRMP error. 
Thus, in Eq. (15) more weight is applied to STRMP errors that have the smallest spread of the cloud along their direction.

Fig. 14 depicts three mesh plots for different ranges of values in the weighting function. The range of each variable is $r_{s}=[0,1.5], \theta=\left[0, \frac{\pi}{2}\right]$, and $r_{\sigma}=[0,1]$. In the left plot $r_{s}$ is held at a constant value of .5 while $\theta$ and $r_{\sigma}$ are varied. In the middle plot $\theta$ is held at a constant value of $\frac{\pi}{4}$ while $r_{s}$ and $r_{\sigma}$ are varied. In the right plot $r_{\sigma}$ is held at a constant value of .5 while $r_{s}$ and $\theta$ are varied.

As can be seen from Fig. 14, as $r_{\sigma} \rightarrow 1, \sigma_{2} \rightarrow \sigma_{1}$. This means that the cloud of nearest neighbors approaches more of a circular shape. Therefore, more weight is applied when $r_{\sigma} \approx 1$. As $\theta \rightarrow 90^{\circ}, \widehat{e}_{R}$ becomes orthogonal to the first singular vector resulting in the spread of nearest neighbors to be perpendicular to $\widehat{e}_{R}$. This results in less modeling error so there is more weight applied. Lastly, as $r_{s} \rightarrow 1$ the first singular value and the length of $\widehat{e}_{R}$ approach the same value. The smaller the value of $r_{s}$, the smaller the applied weight since the cloud of nearest neighbors is larger than the STRMP error.

As will be seen in the results, the weighting does slightly improve the results over all experiments. In cases where there is significant error in the local linear model, a small amount of weight will be applied such that the results will not be skewed. However, if a small amount of error is introduced into the local linear model, a much greater amount of weight will be applied for that point. 

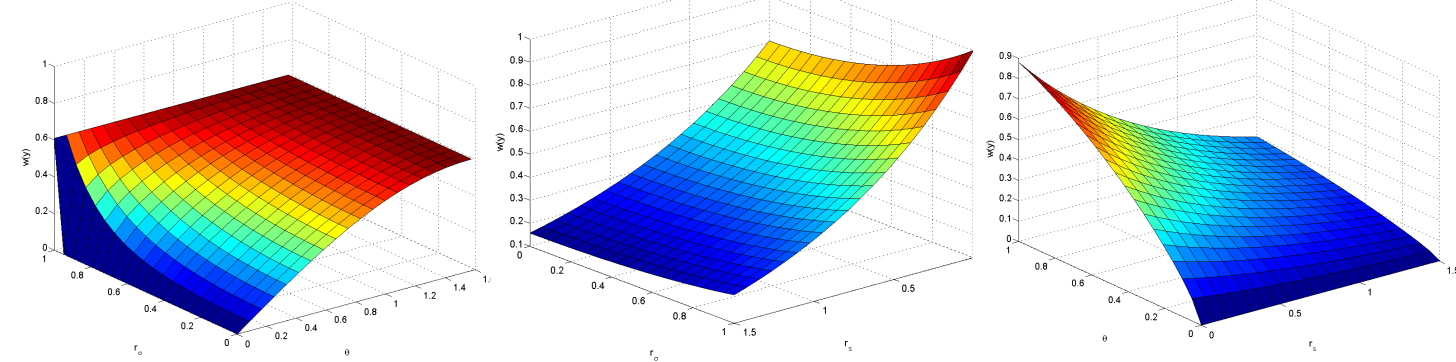

Figure 14. Mesh plots showing three different scenarios for possible values of the weighting function held constant. Left plot: $r_{s}=.5$, Middle plot: $\theta=\frac{\pi}{4}$, Right plot: $r_{\sigma}=.5$. 


\section{CHAPTER 3}

\section{Smooth Orthogonal Decomposition}

Smooth orthogonal decomposition (SOD) $[12,13,8,14]$ is a multivariate data analysis method which can be thought of as an extension of proper orthogonal decomposition (POD) $[16,17,18,19,32,6,14,12,8,13,20,33,15]$. POD is also know as Karhunen-Loev́e Decomposition [16, 30], singular value decomposition [16], etc., and is widely used for model reduction. POD is used to obtain the best low dimensional approximation of a high dimensional data set in the least squares sense, by considering only spatial (i.e., statistical) characteristics.

In contrast, SOD is aimed at obtaining the 'smoothest' low dimensional approximation of a high dimensional dynamical process by taking into consideration the temporal (i.e., dynamical) characteristics as well. If we consider a high dimensional system that contains some deterministic (i.e., smooth in time) signals, SOD looks for projections that are smoothest in time.

For example, consider the multivariate data set which is illustrated as the entangled mass of black lines in Fig. 15 which contains some embedded deterministic trend. By applying SOD to this data set we can extract the smooth deterministic trend. For illustration purposes, if the smooth trend is a sine wave then in a two-dimensional subspace, the extracted trend is a circle. In a one-dimensional subspace, we can fully extract the embedded signal.

SOD takes a multivariate data set and transforms it into a new coordinate system. The basis of this new coordinate system is such that when the data is projected onto the basis vectors, the time functions are as smooth as possible. The basis vectors are referred to as smooth projection modes (SPMs) $\{\psi(x)\}_{i=1}^{p}$

and the time functions are smooth orthogonal coordinates (SOCs) $\{q(t)\}_{i=1}^{p}$, where 


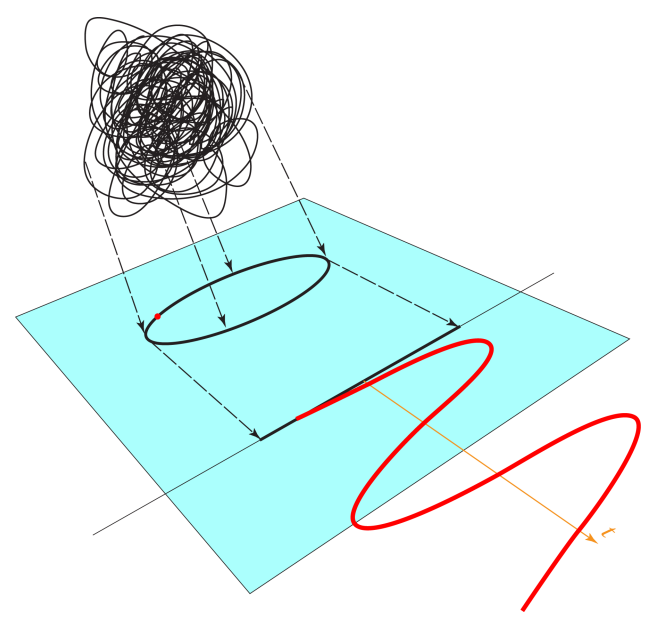

Figure 15. Graphical illustration of using SOD to extract a smooth deterministic trend (sine wave) which is embedded in a multivariate data set.
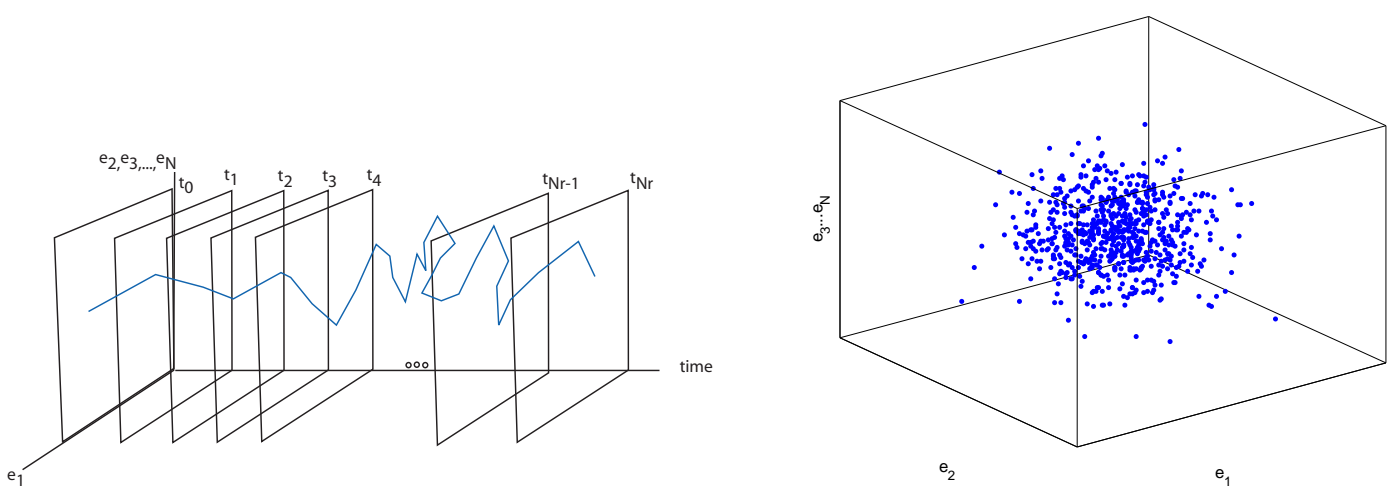

Figure 16. (Left) A deterministic trend embedded int a (Right) high dimensional feature space.

$p$ is the dimension of the data set.

Figs. 16 and 17 depict a graphical illustration of the SOD procedure. In Fig. 16 (Right), a multivariate data set contains the time trajectory that is shown in Fig. 16 (Left). The objective of SOD is to extract the smooth deterministic trajectory from this multivariate data set. To do so, SOD will identify a set of SPMs from the data. Here, three SPMs are determined and labeled in Fig. 17 (Left). These SPMs shown as different colors for the sake of clarity. Furthermore, the extracted SOCs (smooth trajectory) are shown in Fig. 17 (Right).

In this chapter, the continuous formulation of SOD will be presented and 

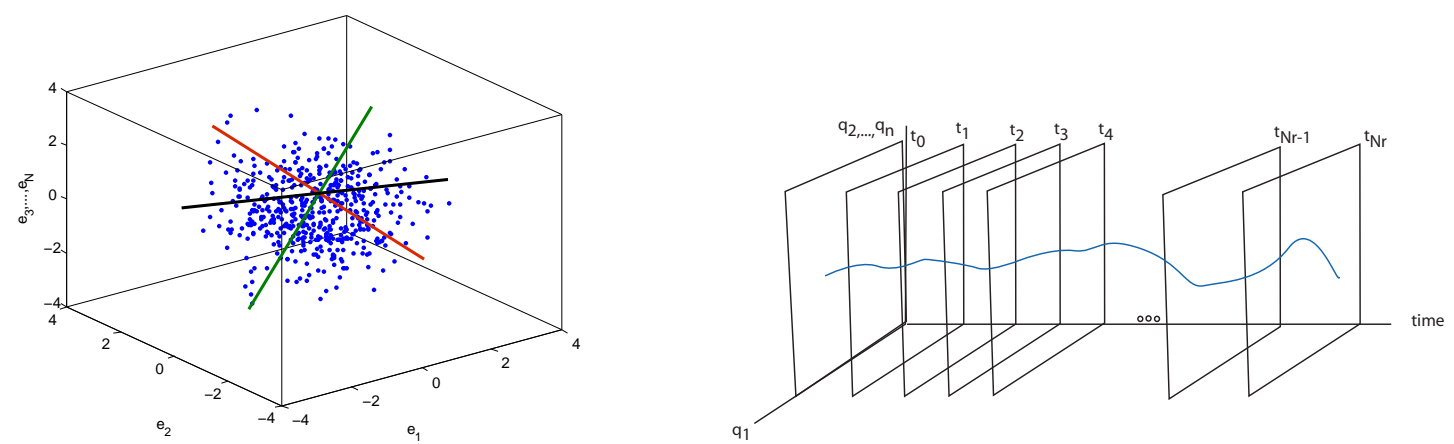

Figure 17. (Left) The first three new basis coordinates found from applying SOD to the multivariate data set. The new basis is such that smooth coordinates have maximum variance and minimal local functions. (Right) Smooth coordinate extracted from the multivariate data set using SOD.

followed by the discrete case derivation. Once the linear SOD formulation is presented, a nonlinear extension (quadratic and cubic nonlinear expansions) will be explored. After the formulation of SOD has been presented, several properties including: invariance to a linear transform and orthogonality of SPMs and SOCs will be presented. Finally, some applications including: recovering harmonic signals contaminated with white noise and approximating linear normal modes from a discrete and a continuous system will be presented.

\subsection{SOD-Continuous Formulation}

Suppose that we have a function (field) $y(x, t) \in \mathbb{R}^{m}$ that we wish to approximate over some domain $\Theta$. If $x \in \Theta \subset \mathbb{R}^{n}$ is a vector-valued spatial coordinate and $t \in \mathrm{T} \subset \mathbb{R}$ is a scalar temporal coordinate, we can approximate $y(x, t)$ as a finite sum in the variables separated form,

$$
y(x, t) \approx \sum_{j=1}^{N} q_{j}(t) \phi_{j}(x) .
$$

The temporal coordinates $q_{k}(t) \in \mathbb{R}$ and spatial modes $\phi_{k}(x) \in \mathbb{R}^{m}$ are not unique. We can think of $y(x, t)$ as a map where $y(x, t): \mathbb{R} \times \mathbb{R}^{n} \mapsto \mathbb{R}^{m}$. If $x$ is bound on some interval $\Theta \subset \mathbb{R}^{n}$ there are several possible choices for the function $\phi_{k}(x)$. These 
include orthogonal Fourier series, Legendre polynomials, Chebyshev polynomials, etc.

In the case of POD, $\left\{\phi_{k}(x)\right\}_{k=1}^{N}$ is chosen to be a set of orthonormal basis functions such that the approximation of $y(x, t)$ for each $N$ is as good as possible in the least squares sense. Therefore, POD finds the sequence of orthonormal basis functions $\phi_{k}(x)$ for which the first $k$ of these functions give the best possible $k$ term approximation.

The $k^{\text {th }}$ SPM, $\psi_{k}(x)$, is defined as the inverse of the smooth orthogonal mode $\phi_{j}(x)$, which is expressed mathematically as,

$$
\int_{\Theta} \psi_{k}^{T}(x) \phi_{j}(x) d x=\alpha_{k} \delta_{k j}
$$

Pre-multiplying Eq. (21) by the $k^{\text {th }}$ smooth projection mode we obtain,

$$
\psi_{k}^{T}(x) y(x, t) \approx \sum_{j=1}^{N} q_{j}(t) \psi_{k}^{T}(x) \phi_{j}(x)
$$

Integrating both sides of Eq. (23) over the domain $\Theta$ we are left with,

$$
\int_{\Theta} \psi_{k}^{T}(x) y(x, t) d x \approx \int_{\Theta} \sum_{j=1}^{N} q_{j}(t) \psi_{k}^{T}(x) \phi_{j}(x) d x .
$$

It is important to note that the $k^{\text {th }} \mathrm{SPM} \psi_{k}(x)$ is orthogonal to the subspace formed by all but the $k^{\text {th }}$ smooth orthogonal mode or $\psi_{k}(x)$ is orthogonal to the null space of the $k^{\text {th }}$ smooth orthogonal mode,

$$
\psi_{k}(x) \perp \operatorname{span}\left\{\bigcup_{\phi_{i}(x) \neq \phi_{k}(x)} \phi_{j}(x)\right\} \perp \operatorname{null}\left\{\phi_{k}(x)\right\}
$$

where $\bigcup(\cdot)$ represents the union.

The right hand side of Eq. (24) reduces to,

$$
\sum_{j=1}^{N} q_{j}(t) \int_{\Theta} \psi_{k}^{T}(x) \phi_{j}(x) d x=q_{k}(t) .
$$


Finally, we can write the function describing the temporal coordinate as

$$
q_{k}(t)=\int_{\Theta} \psi_{k}^{T}(x) y(x, t) d x
$$

It will be shown later in the section 'Properties of SOD' that the temporal coordinates are orthogonal to each other.

In order to extract the 'smoothest' low dimensional subspace, two constraints are placed on the temporal coordinates. Namely, the variability of the first time derivative of the coordinate $q(t)$ must be minimized while $q(t)$ still retains the maximal variance. This requires the best possible 'smooth' $k$-term approximation of $y(x, t)$ in a least squares sense. Mathematically, this can be formulated as a constrained maximization problem.

The maximization problem can be stated as,

$$
\arg \max _{\psi_{k}}\|q(t)\|^{2} \quad \text { subject to } \quad \arg \min _{\psi_{k}}\|\dot{q}(t)\|^{2}
$$

This can be rewritten in a more familiar form,

$$
\arg \max _{\psi_{k}}\left[\lambda_{k}=\frac{\left\langle q_{k}(t)^{2}\right\rangle}{\left\langle\dot{q}_{k}(t)^{2}\right\rangle}\right]=\arg \max _{\psi_{k}}\left[\lambda_{k}=\frac{\left\langle q_{k}(t)^{2}\right\rangle}{\left\langle\left(D q_{k}\right)(t)^{2}\right\rangle}\right], \forall x \in \Theta, \forall t \in \mathrm{T},
$$

where $D$ is a differential operator and $\langle\cdot\rangle=\frac{1}{T} \int_{T}(\cdot) d t$ is the expected value. We can expand the numerator in Eq. (29) as,

$$
\begin{aligned}
\left\langle q_{k}(t)^{2}\right\rangle & =\left\langle\int_{\Theta} \psi_{k}^{T}(x) y(x, t) d x \int_{\Theta} \psi_{k}^{T}(z) y(z, t) d z\right\rangle \\
& =\frac{1}{|T|} \int_{T}\left[\int_{\Theta} \psi_{k}^{T}(x) y(x, t) d x \int_{\Theta} \psi_{k}^{T}(z) y(z, t) d z\right] d t
\end{aligned}
$$

Changing the order of integration Eq. (30) becomes,

$$
\int_{\Theta} \int_{\Theta} \psi_{k}^{T}(x)\left[\frac{1}{|T|} \int_{T} y(x, t) y^{T}(z, t) d t\right] \psi_{k}(z) d x d z
$$

As long as $y(x, t)$ is zero mean, then the covariance matrix is defined as

$$
\Sigma_{y y}(x, z)=\frac{1}{|T|} \int_{T} y(x, t) y^{T}(z, t) d t
$$


Eq. (30) can be written as

$$
\left\langle q_{k}(t)^{2}\right\rangle=\int_{\Theta} \int_{\Theta} \psi_{k}^{T}(x) \Sigma_{y y}(x, z) \psi_{k}(z) d x d z
$$

Applying the same procedure, we can write the denominator in Eq. (29) as,

$$
\left\langle\left(D q_{k}\right)(t)^{2}\right\rangle=\int_{\Theta} \int_{\Theta} \psi_{k}^{T}(x) \Sigma_{\dot{y} \dot{y}}(x, z) \psi_{k}(z) d x d z
$$

Substituting Eqs. (32), and (33) into Eq. (29) results in,

$$
\arg \max _{\psi_{k}}\left[\lambda_{k}\left(\psi_{k}\right)=\frac{\int_{\Theta} \int_{\Theta} \psi_{k}^{T}(x) \Sigma_{y y}(x, z) \psi_{k}(z) d x d z}{\int_{\Theta} \int_{\Theta} \psi_{k}^{T}(x) \Sigma_{\dot{y} \dot{y}}(x, z) \psi_{k}(z) d x d z}\right] .
$$

One way to solve a constrained maximization problem is to use the Lagrangian multiplier method. In this problem, we wish to find the maxima of $f\left(\psi_{k}\right)=\left\langle q_{k}(t)^{2}\right\rangle$ where the constrained function $g\left(\psi_{k}\right)=\left\langle\left(D q_{k}\right)(t)^{2}\right\rangle=1$ is minimized. Using the method of Lagrangian multipliers, we define an objective function called the Lagrangian to be,

$$
\mathcal{L}\left(\psi_{k}, \lambda_{k}\right)=f\left(\psi_{k}\right)-\lambda_{k}\left(g\left(\psi_{k}\right)-1\right)
$$

where $\lambda_{k}$ is the Lagrange multiplier for the $k^{\text {th }}$ projection mode. Plugging in values for $f\left(\psi_{k}\right)$ and $g\left(\psi_{k}\right)$ we are left with the Lagrangian,

$$
\mathcal{L}\left(\psi_{k}, \lambda_{k}\right)=\left\langle q_{k}(t)^{2}\right\rangle-\lambda_{k}\left(\left\langle\left(D q_{k}\right)(t)^{2}\right\rangle-1\right),
$$

and in its final form,

$$
\begin{aligned}
\mathcal{L}\left(\psi_{k}, \lambda_{k}\right)= & \frac{1}{2} \int_{\Theta} \psi_{k}^{T}(x) \int_{\Theta} \Sigma_{y y}(x, z) \psi_{k}(z) d z d x- \\
& \frac{1}{2} \lambda_{k}\left(\int_{\Theta} \psi_{k}^{T}(x) \int_{\Theta} \Sigma_{\dot{y} \dot{y}}(x, z) \psi_{k}(z) d z d x-1\right) .
\end{aligned}
$$

Solving $\nabla \mathcal{L}\left(\psi_{k}, \lambda_{k}\right)=0$ will provide the needed SPMs $\psi_{k}$ and $\lambda_{k}$. It should be noted that $\nabla_{\psi_{k}(\alpha)} \mathcal{L}\left(\psi_{k}, \lambda_{k}\right)$ is a functional derivative, which can be written as:

$$
\frac{\partial \mathcal{L}\left[\psi_{k}, \lambda_{k}\right]}{\partial\left[\psi_{k}(\alpha)\right]} \triangleq \lim _{\varepsilon \rightarrow 0} \frac{\mathcal{L}\left[\psi_{k}+\varepsilon \delta(\star-\alpha), \lambda_{k}\right]-\mathcal{L}\left[\psi_{k}, \lambda_{k}\right]}{\varepsilon}=0,
$$


where $\star$ indicates the corresponding argument of the $\psi_{k}$ function, $\delta$ is the Dirac delta function, and $\alpha$ is the point to which the functional derivative to taken about. After substituting the functions into Eq. (38), the equation reduces too,

$$
\begin{aligned}
& =\lim _{\varepsilon \rightarrow 0}\left[\frac{\int_{\Theta}\left[\psi_{k}^{T}(x)+\varepsilon \delta^{T}(x-\alpha)\right] \int_{\Theta} \Sigma_{y y}(x, z)\left[\psi_{k}(z)+\varepsilon \delta(z-\alpha)\right] d z d x}{2 \varepsilon}\right. \\
& -\frac{\lambda_{k}\left(\int_{\Theta}\left[\psi_{k}^{T}(x)+\varepsilon \delta^{T}(x-\alpha)\right] \int_{\Theta} \Sigma_{\dot{y} \dot{y}}(x, z)\left[\psi_{k}(z)+\varepsilon \delta(z-\alpha)\right] d z d x-1\right)}{2 \varepsilon} \\
& \left.-\frac{\int_{\Theta} \psi_{k}^{T}(x) \int_{\Theta} \Sigma_{y y}(x, z) \psi_{k}(z) d z d x-\lambda_{k}\left(\int_{\Theta} \psi_{k}^{T}(x) \int_{\Theta} \Sigma_{\dot{y} \dot{y}}(x, z) \psi_{k}(z) d z d x-1\right)}{2 \varepsilon}\right]=0 \\
& =\lim _{\varepsilon \rightarrow 0}\left[\frac{\int_{\Theta}\left[\psi_{k}^{T}(x)+\varepsilon \delta^{T}(x-\alpha)\right] \int_{\Theta} \Sigma_{y y}(x, z)\left[\psi_{k}(z)+\varepsilon \delta(z-\alpha)\right] d z d x}{2 \varepsilon}\right. \\
& -\frac{\int_{\Theta} \psi_{k}^{T}(x) \int_{\Theta} \Sigma_{y y}(x, z) \psi_{k}(z) d z d x}{2 \varepsilon} \\
& -\lambda_{k} \frac{\int_{\Theta}\left[\psi_{k}^{T}(x)+\varepsilon \delta^{T}(x-\alpha)\right] \int_{\Theta} \Sigma_{\dot{y} \dot{y}}(x, z)\left[\psi_{k}(z)+\varepsilon \delta(z-\alpha)\right] d z d x}{2 \varepsilon} \\
& \left.\lambda_{k} \frac{-\int_{\Theta} \psi_{k}^{T}(x) \int_{\Theta} \Sigma_{\dot{y} \dot{y}}(x, z) \psi_{k}(z) d z d x}{2 \varepsilon}\right]=0 \\
& =\lim _{\varepsilon \rightarrow 0}\left[\frac{\int_{\Theta} \psi_{k}^{T}(x) \int_{\Theta} \Sigma_{y y}(x, z) \delta(z-\alpha) d z d x+\int_{\Theta} \delta^{T}(x-\alpha) \int_{\Theta} \Sigma_{y y}(x, z) \psi_{k}^{T}(z) d z d x}{2}\right. \\
& -\frac{\lambda_{k}\left(\int_{\Theta} \psi_{k}^{T}(x) \int_{\Theta} \Sigma_{\dot{y} \dot{y}}(x, z) \delta(z-\alpha) d z d x+\int_{\Theta} \delta(x-\alpha) \int_{\Theta} \Sigma_{\dot{y} \dot{y}}(x, z) \psi_{k}(z) d z d x\right)}{2} \\
& +\frac{\varepsilon}{2}\left(\int_{\Theta} \delta^{T}(x-\alpha) \int_{\Theta} \Sigma_{y y}(x, z) \delta(z-\alpha) d z d x\right) \\
& \left.+\frac{\varepsilon}{2}\left(\int_{\Theta} \delta^{T}(x-\alpha) \int_{\Theta} \Sigma_{\dot{y} \dot{y}}(x, z) \delta(z-\alpha) d z d x\right)\right]=0 \\
& =\frac{\int_{\Theta} \psi_{k}^{T}(x) \int_{\Theta} \Sigma_{y y}(x, z) \delta(z-\alpha) d z d x+\int_{\Theta} \delta^{T}(x-\alpha) \int_{\Theta} \Sigma_{y y}(x, z) \psi_{k}^{T}(z) d z d x}{2} \\
& -\frac{\lambda_{k}\left(\int_{\Theta} \psi_{k}^{T}(x) \int_{\Theta} \Sigma_{\dot{y} \dot{y}}(x, z) \delta(z-\alpha) d z d x+\int_{\Theta} \delta(x-\alpha) \int_{\Theta} \Sigma_{\dot{y} \dot{y}}(x, z) \psi_{k}(z) d z d x\right)}{2}=0 .
\end{aligned}
$$

Since the integrals are symmetric, the equation can reduce to

$$
\int_{\Theta} \delta^{T}(x-\alpha) \int_{\Theta} \Sigma_{y y}(x, z) \psi_{k}^{T}(z) d z d x-\lambda_{k} \int_{\Theta} \delta(x-\alpha) \int_{\Theta} \Sigma_{\dot{y} \dot{y}}(x, z) \psi_{k}(z) d z d x=0 .
$$

Therefore, we are left a generalized integral eigenvalue problem defining SOD for $y(x, t)$ as

$$
\int_{\Theta} \Sigma_{y y}(x, z) \psi_{k}(z) d z=\lambda_{k} \int_{\Theta} \Sigma_{\dot{y} \dot{y}}(x, z) \psi_{k}(z) d z, \quad \forall x \in \Theta
$$


and

$$
\int_{\Theta} \psi_{k}^{T}(x) \int_{\Theta} \Sigma_{\dot{y} \dot{y}}(x, z) \psi_{k}(z) d z d x=1,
$$

where $\lambda_{k}$ are generalized eigenvalues or smooth orthogonal values (SOVs) and $\psi_{k}$ are generalized eigenvectors or smooth projection modes (SPMs). By projecting our matrix $Y$ onto the SPMs we obtain smooth orthogonal coordinates (SOCs).

\subsection{SOD-Discrete Formulation}

The discrete formulation of SOD follows directly from the continuous case. Consider a dynamical system of which $n$ state variables are measured. In addition, each $n$ variable is sampled $m$ times, which are arranged into a $m \times n$ ensemble matrix $Y$. If the $n$ state variables are $n$ spatial locations, then the $Y_{i j}$ element represents the $i^{\text {th }}$ time instant value for the $j^{\text {th }}$ spatial location.

Next, an ensemble of each time-derivatives of the state variable is formed by $\dot{Y} \approx D Y$. To calculate the time derivative we use a differential operator based on forward difference, however, we could have used the Jacobian or some other differential technique. The differential operator $D \in \mathbb{R}^{(n-1) \times n}$ is,

$$
D=\frac{1}{\Delta t}\left[\begin{array}{rrrrrc}
-1 & 1 & 0 & 0 & \cdots & 0 \\
0 & -1 & 1 & 0 & \cdots & 0 \\
\vdots & \ddots & \ddots & \ddots & \ddots & \vdots \\
0 & \cdots & 0 & -1 & 1 & 0 \\
0 & \cdots & 0 & 0 & -1 & 1
\end{array}\right]
$$

Provided that $Y$ and $\dot{Y}$ are zero mean, the auto-covariance matrices can be formed by

$$
\Sigma_{y y}=\frac{1}{N-1} Y^{T} Y, \quad \Sigma_{\dot{y} \dot{y}}=\frac{1}{N-2} \dot{Y}^{T} \dot{Y}
$$

where $N$ is the number of points and $\Sigma_{y y}, \Sigma_{\dot{y} \dot{y}} \in \mathbb{R}^{n \times n}$. We divide by $N-1$ and not $N$ because the covariance matrices are sample covariance matrices. ${ }^{1}$

\footnotetext{
${ }^{1}$ When using collected data to construct the covariance matrix, the sample covariance matrix is used. As above, the sample covariance matrix is estimated as, $\operatorname{cov}(X, Y)=\frac{1}{N-1}(X-\bar{X})(Y-\bar{Y})$, where $\bar{X}$ and $\bar{Y}$ are the sample means of the random variable $X$ and $Y$, respectively. Therefore,
} 
In the finite dimensional discrete case the analog to Eq. (21) is,

$$
Y=Q \Phi^{T}
$$

where $Q=\left[q_{1}, q_{2}, \cdots, q_{n}\right] \in \mathbb{R}^{m \times n}$ is a matrix composed of SOCs and $\Phi=$ $\left[\phi_{1}, \phi_{2}, \cdots, \phi_{n}\right] \in \mathbb{R}^{n \times n}$ is a matrix composed of SOMs. For the discrete case, the analog to Eq. (35) is,

$$
\Sigma_{y y} \psi_{k}=\lambda_{k} \Sigma_{\dot{y} \dot{y}} \psi_{k}
$$

where $\lambda_{k}$ are SOVs and $\psi_{k} \in \mathbb{R}^{n}$ are SPMs.

Generally, the solution to Eq. (43) is obtained using generalized singular value decomposition of the matrix pair $\Sigma_{y y}$ and $\Sigma_{\dot{y} \dot{y}}$, yielding:

$$
\begin{gathered}
Y=Q \Phi^{T}=Q \Psi^{-1}, \\
D Y=V S \Phi^{T}, \\
C^{T} C+S^{T} S=I,
\end{gathered}
$$

where $I$ is the identity matrix, the matrices $U$ and $V$ are unitary, $\Phi$ is a square matrix, and $C$ and $S$ are non-negative diagonal matrices. $\Psi=\left[\psi_{1}, \psi_{2}, \ldots, \psi_{N_{e}}\right]=\Phi^{-T}$ is a matrix composed of SPMs. The columns of matrix $Q=U C$ contain SOCs, and the corresponding eigenvalues or SOVs are given by term-by-term division of $\operatorname{diag}\left(C^{T} C\right) / \operatorname{diag}\left(S^{T} S\right)$. The degree of smoothness of the coordinates are described by the magnitude of the corresponding smooth values. Thus, the greater in magnitude the SOV, the smoother in time is the corresponding coordinate. It should be noted that if we were to replace $\Sigma_{\dot{y} \dot{y}}$ with the identity matrix, then the formulation would yield the proper orthogonal decomposition.

$N-1$ is used instead of $N$ because we may only have access to the sample mean and not he actual population mean $E[X]$ and $E[Y]$. However, if the population mean is known, then the estimated covariance becomes, $\operatorname{cov}(X, Y)=\frac{1}{N}(X-E[X])(Y-E[Y])$. 


\subsection{Nonlinear SOD}

Nonlinear SOD is achieved by the polynomial expansion of the smooth coordinates obtained from a subspace of the vector-valued feature space. This subspace contains $95 \%$ of the energy of the feature space and is identified through POD. The feature space is projected onto a set of proper orthogonal modes that capture $95 \%$ of the energy. Therefore, the most significant amount of noise is removed from the feature space before applying SOD. Now, the nonlinear SOD can be applied to the smooth coordinates extracted from linear SOD of the subspace.

The first $p$-dimensional dominant modes (identified by the dominant SOVs) are used to extract the first $p$ smooth coordinates, $\left\{q_{i}\right\}_{i=1}^{p}$, where a polynomial expanded to include nonlinear terms. In the polynomial expansion, we include both quadratic and cubic terms. A new vector-valued time series including quadratic

terms of the second-order polynomial expansion of the linear SOCs is obtained by

$$
Y^{(2)}=[\underbrace{q_{1}, \ldots, q_{p}}_{1^{\text {st }} \text { order }}, \underbrace{q_{1} \cdot q_{1}, q_{1} \cdot q_{2}, \ldots, q_{1} \cdot q_{p}, \ldots, q_{p} \cdot q_{p}}_{2^{\text {nd }} \text { order }}],
$$

where $(\cdot)$ refers to term-by-term multiplication of the arrays. We can write the third-order expansion as

$$
Y^{(3)}=[Y^{(2)}, \underbrace{q_{1} \cdot q_{1} \cdot q_{1}, q_{1} \cdot q_{1} \cdot q_{2}, \ldots, q_{1} \cdot q_{1} \cdot q_{p}, \ldots, q_{p} \cdot q_{p} \cdot q_{p}}_{3^{\text {rd }} \text { order }}] .
$$

From here, we can obtain nonlinear SOCs (NSOCs) through the generalized singular value decomposition or perform linear SOD on the matrix pairs $Y^{(2)}$ and $D Y^{(2)}$ or $Y^{(3)}$ and $D Y^{(3)}$.

Since each expansion has as a subset of the lower order approximations, the result of the higher order approximation can be at a minimum as best as the lower order approximation (using only $p$ SOCs). For instance, in a quadratic expansion, the results have to be as best as the linear approximation provided the same number of SOCs are used. If all of the SOCs in linear SOD are used and only 
five SOCs in the quadratic expansion, the linear SOD will most likely yield a far better result since all coordinates are being used. Therefore, any slight change in the trends of the NSOCs will suggest improvement (smoother in time) of the nonlinear coordinates provided the same number of SOCs are used.

\subsection{Properties of SOD}

\subsubsection{Invariance to invertible coordinate transforms}

The first property of SOD is its invariance to a coordinate transformation, which was demonstrated in [32]. In the SOD formulation, we have the ensemble matrix $Y$ and $D Y$. If we propose a coordinate transformation, namely, $Y=X R$ and $D Y=D X R$ where $R \in \mathbb{R}^{m \times m}$ is an invertible matrix, we can show that the SOCs from $X R$ and $D X R$ yield the same result. Therefore, we can make the assertion that SOD is invariant to an invertible linear transformation.

Taking the definition of our covariance matrix for $Y$ we had $\frac{Y^{T} Y}{N-1}$. Substituting in the transformation $Y=X R$ we have,

$$
\frac{Y^{T} Y}{N-1}=\frac{(X R)^{T}(X R)}{N-1}=\frac{R^{T} X^{T} X R}{N-1}=\frac{R^{T} \Sigma_{x x} R}{N-1} .
$$

For the covariance matrix $D Y$ we had $\frac{(D Y)^{T} D Y}{N-2}$. Substituting in the transformation $D Y=D X R$ we have,

$$
\frac{(D Y)^{T} D Y}{N-2}=\frac{(D X R)^{T}(D X R)}{N-2}=\frac{R^{T} X^{T} D^{T} D X R}{N-2}=\frac{R^{T} \Sigma_{\dot{x} \dot{x}} R}{N-2} .
$$

Plugging in the values for the covariance matrix in the SOD equation Eq. (43) we have,

$$
R^{T} \Sigma_{x x} R \hat{\psi}_{k}=\hat{\lambda}_{k} R^{T} \Sigma_{\dot{x} \dot{x}} R \hat{\psi}_{k}
$$

If we left multiply Eq. (49) by $R^{-T}$ we obtain,

$$
R^{-T} R^{T} \Sigma_{x x} R \hat{\psi}_{k}=\hat{\lambda}_{k} R^{-T} R^{T} \Sigma_{\dot{x} \dot{x}} R \hat{\psi}_{k}
$$

which reduces to the final transformed equation,

$$
\Sigma_{x x} R \hat{\psi}_{k}=\hat{\lambda}_{k} \Sigma_{\dot{x} \dot{x}} R \hat{\psi}_{k}
$$


where

$$
\hat{\psi}_{k}=R^{-1} \psi_{k}
$$

The columns of $\hat{\psi}$ contain the transformed SPMs and the SOCs are $q=X \psi$.

\subsubsection{Orthogonality of projection modes and smooth coordinates}

In this section, it will be shown for both the discrete and continuous case, that smooth projection modes $\left\{\psi_{i}\right\}_{i=1}^{p}$ are orthogonal to the covariance matrices and the smooth coordinates $\left\{q_{i}\right\}_{i=1}^{p}$ are orthogonal to each other.

First, we consider the discrete case.

It was shown previously in Eq. (43) that

$$
\Sigma_{y y} \psi_{k}=\lambda_{k} \Sigma_{\dot{y} \dot{y}} \psi_{k}
$$

If we pre-multiply both sides of the Eq. (53) by $\psi_{j}^{T}$ we get,

$$
\psi_{j}^{T} \Sigma_{y y} \psi_{k}=\lambda_{k} \psi_{j}^{T} \Sigma_{\dot{y} \dot{y}} \psi_{k}
$$

Similarly, for the $j^{\text {th }}$ mode the equation is,

$$
\Sigma_{y y} \psi_{j}=\lambda_{j} \Sigma_{\dot{y} \dot{y}} \psi_{j}
$$

If we pre-multiply Eq. (55) by $\psi_{k}^{T}$ we get,

$$
\psi_{k}^{T} \Sigma_{y y} \psi_{j}=\lambda_{j} \psi_{k}^{T} \Sigma_{\dot{y} \dot{y}} \psi_{j}
$$

Taking the transpose of each side of Eq. (54) and noting the covariance matrices are symmetric we are left with

$$
\psi_{k}^{T} \Sigma_{y y} \psi_{j}=\lambda_{k} \psi_{k}^{T} \Sigma_{\dot{y} \dot{y}} \psi_{j}
$$

Subtracting Eq. (57) from Eq. (56) results in,

$$
0=\left(\lambda_{k}-\lambda_{j}\right) \psi_{k}^{T} \Sigma_{\dot{y} \dot{y}} \psi_{j} .
$$


Therefore, if $\lambda_{k} \neq \lambda_{j}$ then $\psi_{k}^{T} \Sigma_{\dot{y} \dot{y}} \psi_{j}=0$ and we are finally left with,

$$
\psi_{k}^{T} \Sigma_{y y} \psi_{j}=\alpha_{k} \delta_{k j}
$$

which verifies that the projection modes are orthogonal with respect to the covariance matrix. Now, we are in the position to show that the coordinates are orthogonal. We can define the coordinates to be, $q_{k}=Y \psi_{k}$. As in the derivation, we can express $\left\langle q_{k}^{2}\right\rangle$ as,

$$
\begin{aligned}
\left\langle q_{k}^{2}\right\rangle & =q_{k}^{T} q_{j}=\psi_{k}^{T} Y^{T} Y \psi_{j} \\
& =\psi_{k}^{T} \Sigma_{y y} \psi_{j}(N-1) \\
& =(N-1) \delta_{k j} .
\end{aligned}
$$

For the continuous case the derivation is as follows.

It was previously shown that the generalized integral eigenvalue problem for the $k^{\text {th }}$ mode is written as,

$$
\int_{\Theta} \Sigma_{y y}(x, z) \psi_{k}(z) d z=\lambda_{k} \int_{\Theta} \Sigma_{\dot{y} \dot{y}}(x, z) \psi_{k}(z) d z
$$

Following the same procedure from the discrete case, we are left with the final equation that,

$$
0=\left(\lambda_{k}-\lambda_{j}\right) \int_{\Theta} \psi_{k}^{T}(x) \int_{\Theta} \Sigma_{\dot{y} \dot{y}} \psi_{j}(z) d z d x .
$$

From Eq. (40), if $\lambda_{k} \neq \lambda_{j}$ then $\int_{\Theta} \psi_{k}^{T}(x) \int_{\Theta} \Sigma_{\dot{y} \dot{y}} \psi_{j}(z) d z d x=0$ and we are finally left with,

$$
\int_{\Theta} \psi_{k}^{T}(x) \int_{\Theta} \Sigma_{y y} \psi_{j}(z) d z d x=\alpha_{k} \delta_{k j}
$$

which verifies that the projection modes are orthogonal with respect to the covariance matrix.

Now, to show the coordinates are orthogonal we consider their covariance from Eq. (30),

$$
\frac{1}{|T|} \int_{T} q_{j}(t) q_{k}(t) d t=\frac{1}{|T|} \int_{T}\left[\int_{\Theta} \psi_{j}^{T}(x) y(x, t) d x \int_{\Theta} \psi_{k}^{T}(z) y(z, t) d z\right] d t .
$$


Changing the order of integration we can rewrite Eq. (64) as

$$
\frac{1}{|T|} \int_{T}\left[\int_{\Theta} \psi_{j}^{T}(x) \int_{\Theta} \Sigma_{y y}(x, z) \psi_{k}^{T}(z) d z d x\right] d t
$$

From our orthogonality condition in Eq. (63) we are left with

$$
\frac{1}{|T|} \int_{T} \lambda_{k} \delta_{k j} d t=\lambda_{k} \delta_{k j}
$$

Therefore, we obtain the orthogonality condition for the SOCs

$$
\left\langle q_{j} q_{k}\right\rangle=\lambda_{k} \delta_{k j}
$$

\subsection{Applications of SOD}

\subsubsection{Recovering harmonic signals contaminated with white noise}

If we have access to a purely smooth deterministic signal without noise contamination, then SOD will extract that trend. However, in an experimental case, obtaining a signal of this nature is almost impossible. In experiments, one has to take into consideration measurement noise and dynamical noise. During measurements, noise is added into the recording of data or into the signal. Strain gauges, accelerometers, etc., are a few measurement devices that introduce noise into the recordings. With dynamical noise, the noise may enter the system as random fluctuations of the parameters. In this case, it is not a simple superposition of the signal and noise, but rather a signal modulated by noise.

In what follows, obtained smooth coordinates from SOD of a purely deterministic signal will be shown. Next, smooth coordinates will be extracted from a signal with white gaussian noise, $\mathcal{N}(0,1)$, and then a signal that is pure noise. In the case with no noise, the trends should be extracted cleanly. With a little noise contamination, the signal is still discernable and the coordinates should be extracted and almost match the ideal case. When there is no signal, just noise, there is no trend to extract so the coordinates will show this. 
The first three signals of an ensemble matrix composed of sinusoidal signals are shown in Fig. 18 (Top). The sinusoidal signal is composed of harmonic frequencies $x_{n}=\sin (n \pi t)$ where $n=1, \cdots, 10$. After subtracting the mean from the ensemble matrix, the smooth coordinates are calculated and shown in Fig. 18 (Bottom). Clearly, the signals and the coordinates match each other as expected.
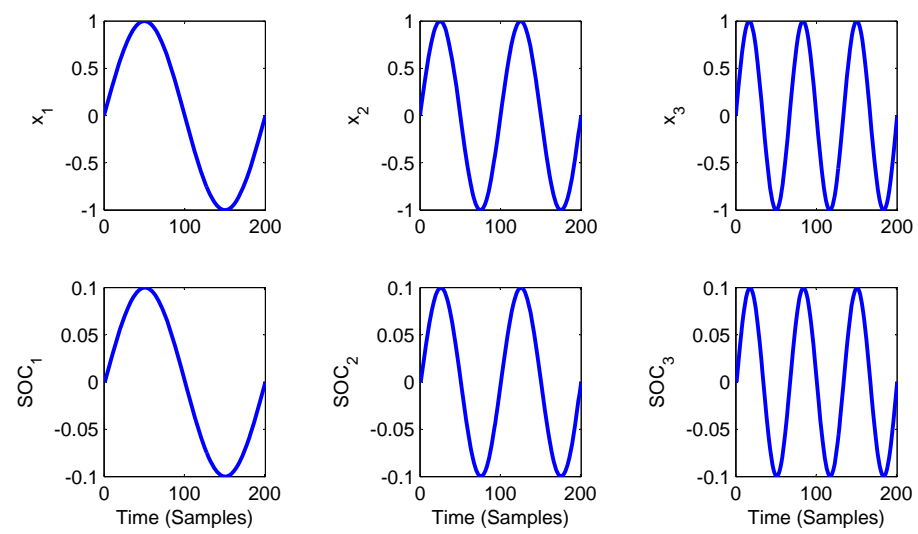

Figure 18. (Top) Sinusoidal signal composed of harmonic frequencies, $x_{n}=$ $\sin (n \pi t)$ and (Bottom) extracted SOCs from ensemble matrix composed of signals. The extracted coordinates match considerably well with the signals.

In the presence of noise, the extracted smooth coordinates should be able to recover the trend. Here, we add white gaussian noise $\mathcal{N}(0,1)$ to our signal $x_{n}=$ $\sin (n \pi t)+\mathcal{N}(0,1)$. Fig. 19 depicts the signals and coordinates. Referring to the signals, there is a tremendous amount of noise which further validates the ability of SOD to recover the embedded sinusoidal signals. A close look at the smooth coordinates demonstrates they are very similar to the coordinates from Fig. 18. Clearly, SOD was able to extract the signal. Take note that the lines are not as smooth and are slightly varied in amplitude, however, in practical situations you will not have a signal with this amount noise. However, this example depicts SOD's ability to extract the coordinates when presented with a signal highly contaminated with noise.

When the signal is composed of just white gaussian noise, there is no deter- 

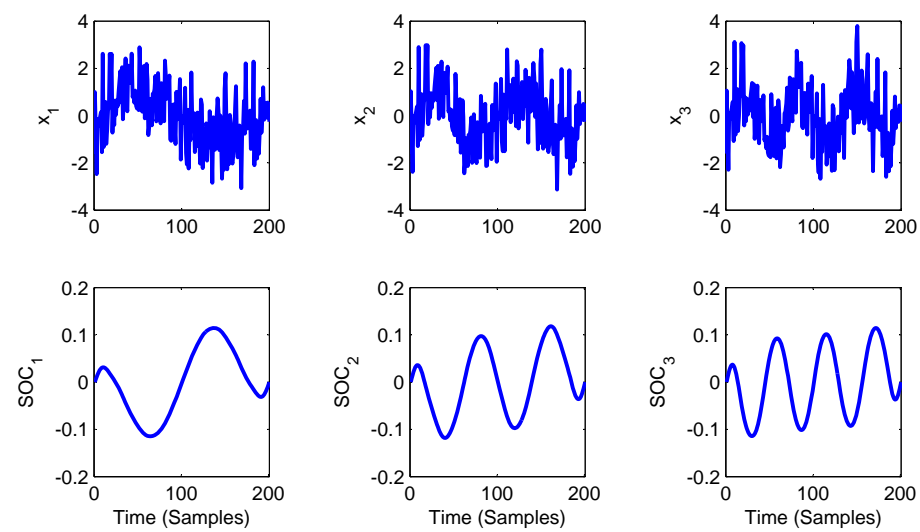

Figure 19. (Top) Sinusoidal signal composed of harmonic frequencies, $x_{n}=$ $\sin (n \pi t)+\mathcal{N}(0,1)$ and (Bottom) extracted SOCs from ensemble matrix composed of signals. The coordinates match considerably well with the signals. There is some slight variation in the coordinates but the structure of the signal is partially recovered.

ministic signal to extract, Fig. 20. For a white gaussian signal, the signal contains equal power at each frequency and therefore the coordinates appear as straight lines.
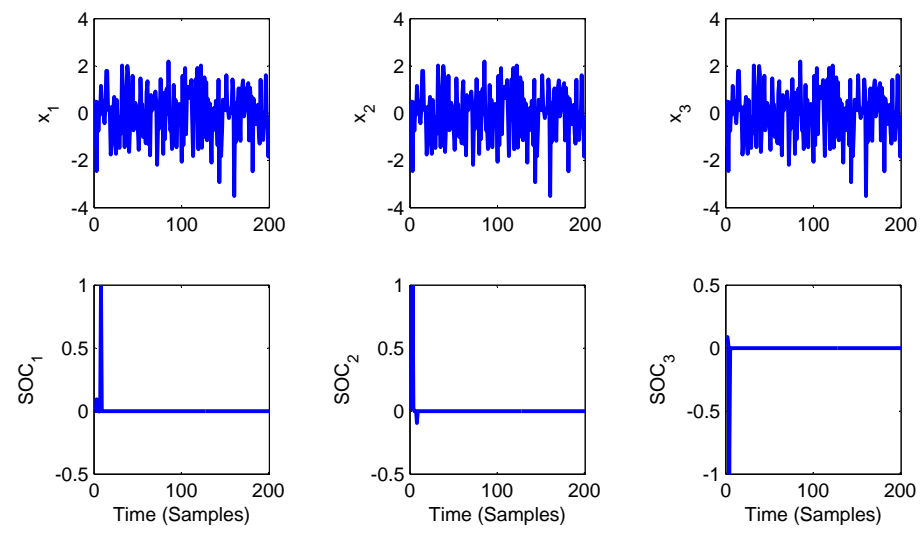

Figure 20. (Top) White noise $\mathcal{N}(0,1)$ and (Bottom) extracted SOCs from ensemble matrix composed of signals. In white noise, there is no signal to extract. 


\subsection{SOD Extracts Linear Normal Modes}

In many linear or linearized nonlinear dynamical systems it may be desirable to calculate the linear normal modes (LNM) of vibration. By knowing the LNM you can fully characterize the oscillating motion of each degree of freedom when all the degrees of freedom move with the same frequency and in phase. These frequencies are known as the natural frequencies. Instead of solving for the LNMs by eigendecomposition - also known as modal analysis - one can approximate the linear modes.

In simple cases, it makes more sense to derive the equations of motion and solve the eigenvalue problem directly for the vibration modes. However, if the system is very complicated to describe by first principles (i.e., many degrees of freedom, coupled structures, etc.) it may not be possible to do so. In addition, often only experimental data is available with some recorded scalar time series from either strain gauges, accelerometers, etc., at node points; calculating the linear normal modes may become very difficult. Here, we demonstrate the versatility of SOD for approximating linear normal modes in both a discrete and a continuous case.

\subsubsection{Approximation of linear normal modes in a lumped mass-spring system}

For the discrete case, consider the five dimensional mass-spring system connected to a rigid surface such as a fixed wall in Fig. 22. The nondimensional mass

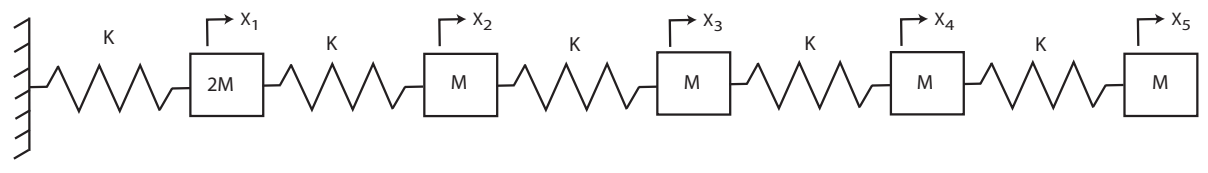

Figure 21. Schematic of mass spring system.

constants are chosen to be $\mathrm{M}=1$ along with the nondimensional stiffness constants 
$\mathrm{K}=1$. The initial conditions are $X(0)=[2,0,0,0,0]^{T}$ and $\dot{X}(0)=[0,0,0,0,0]^{T}$. Using Newton's second law ( $\mathrm{F}=\mathrm{MA})$ the equations of motion are solved for in the form of $2^{\text {nd }}$ order equations of motion,

$$
M \ddot{X}+K X=0 .
$$

The equations of motion for each mass are,

$$
\begin{aligned}
& M_{1} \ddot{X}_{1}=-K_{1} X_{1}+K_{2}\left(X_{1}-X_{2}\right) \\
& M_{2} \ddot{X}_{2}=-K_{2}\left(X_{1}-X_{2}\right)+K_{3}\left(X_{2}-X_{3}\right) \\
& M_{3} \ddot{X}_{3}=-K_{3}\left(X_{2}-X_{3}\right)+K_{4}\left(X_{3}-X_{4}\right) \\
& M_{4} \ddot{X}_{4}=-K_{4}\left(X_{3}-X_{4}\right)+K_{5}\left(X_{4}-X_{5}\right) \\
& M_{5} \ddot{X}_{5}=-K_{5}\left(X_{4}-X_{5}\right) .
\end{aligned}
$$

In matrix form the mass and stiffness matrices become,

$$
M=\left[\begin{array}{lllll}
2 & 0 & 0 & 0 & 0 \\
0 & 1 & 0 & 0 & 0 \\
0 & 0 & 1 & 0 & 0 \\
0 & 0 & 0 & 1 & 0 \\
0 & 0 & 0 & 0 & 1
\end{array}\right] \quad K=\left[\begin{array}{ccccc}
2 & -1 & 0 & 0 & 0 \\
-1 & 2 & -1 & 0 & 0 \\
0 & -1 & 2 & -1 & 0 \\
0 & 0 & -1 & 2 & -1 \\
0 & 0 & 0 & -1 & 1
\end{array}\right]
$$

Fig. 22 depicts the position and velocity time series of the first two masses. The other three degrees of freedom are not presented since the general nature of the plots are the same and would be redundant.

To solve for the linear normal modes and natural frequencies we solve the eigenvalue problem,

$$
\left(K-M \omega^{2}\right)\{\Phi\}=\{0\},
$$

where $\omega^{2}$ are the natural frequencies and $\Phi=\left[\phi_{1}, \phi_{2}, \phi_{3}, \phi_{4}, \phi_{5}\right]$ is a matrix composed of linear normal modes. The natural frequencies are $\omega_{1}=0.2804$, $\omega_{2}=0.7381, \omega_{3}=1.1446, \omega_{4}=1.5795$, and $\omega_{5}=1.8899$. The corresponding 

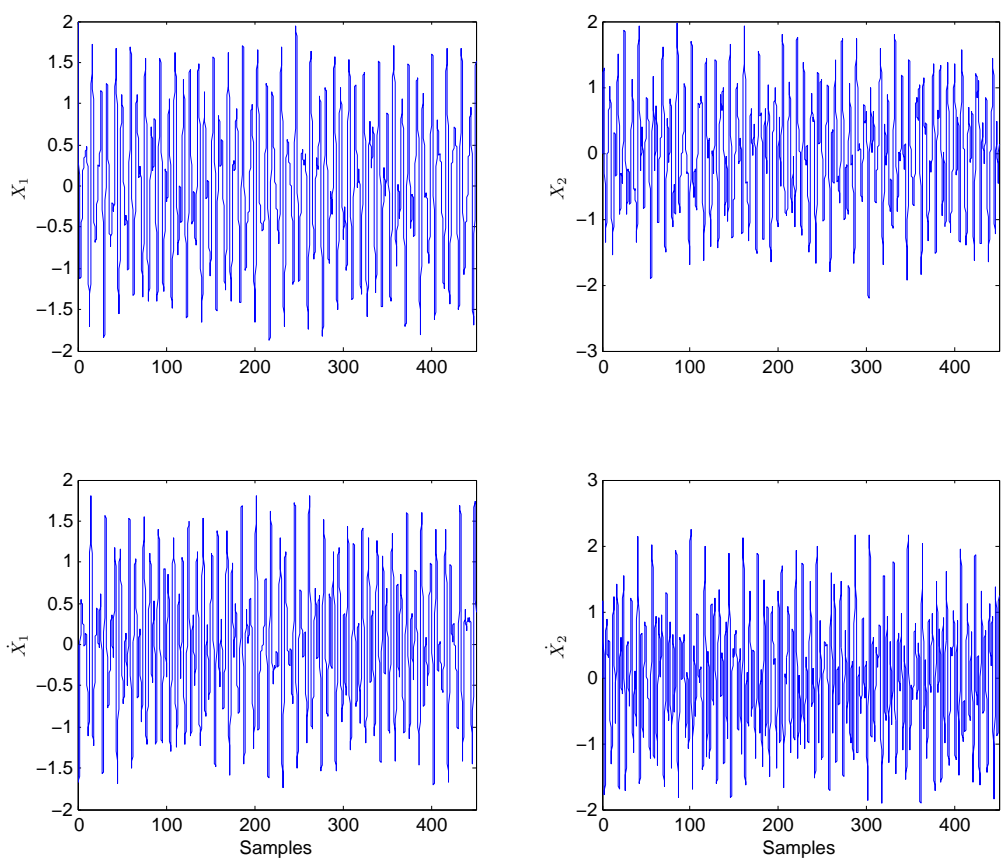

Figure 22. Time series from the simulation of the mass spring system, (Top) displacements $X_{1}$ and $X_{2}$, (Bottom) corresponding velocities $\dot{X}_{1}$ and $\dot{X}_{2}$.

modal matrix composed of linear normal modes is,

$$
\Phi=\left[\begin{array}{ccccc}
0.179 & -0.572 & 0.449 & -0.217 & 0.091 \\
0.331 & -0.521 & -0.27 & 0.6503 & -0.47 \\
0.456 & -0.185 & -0.64 & -0.104 & 0.649 \\
0.546 & 0.2506 & -0.16 & -0.598 & -0.54 \\
0.592 & 0.5506 & 0.529 & 0.4005 & 0.213
\end{array}\right] .
$$

For the SOD, the displacements and velocities from Eq. (69) are used to form their own respective ensemble matrix. The matrices $X, \dot{X} \in \mathbb{R}^{4000 \times 5}$ were constructed and their respective covariance matrices were calculated. The natural frequencies are estimated by the SOVs as $\omega=\frac{1}{\sqrt{\lambda}}$ with $\omega_{1}=0.2794, \omega_{2}=0.7391$, $\omega_{3}=1.1447, \omega_{4}=1.5783$, and $\omega_{5}=1.8907$. The modes are estimated by the smooth orthogonal modes which yield,

$$
\Psi=\left[\begin{array}{ccccc}
0.174 & -0.572 & -0.453 & -0.218 & 0.091 \\
0.323 & -0.520 & 0.279 & 0.650 & -0.477 \\
0.455 & -0.186 & 0.641 & -0.105 & 0.650 \\
0.549 & 0.250 & 0.163 & -0.597 & -0.543 \\
0.596 & 0.551 & -0.526 & 0.401 & 0.211
\end{array}\right]
$$


Fig. 23 depicts graphically the values for the linear normal modes and smooth orthogonal modes. Except for the $4^{\text {th }}$ smooth mode, the modes approximate the LNM almost exactly. It is clear that given a discrete mass spring system, the SOD method can approximate the natural frequencies and modes of vibration.
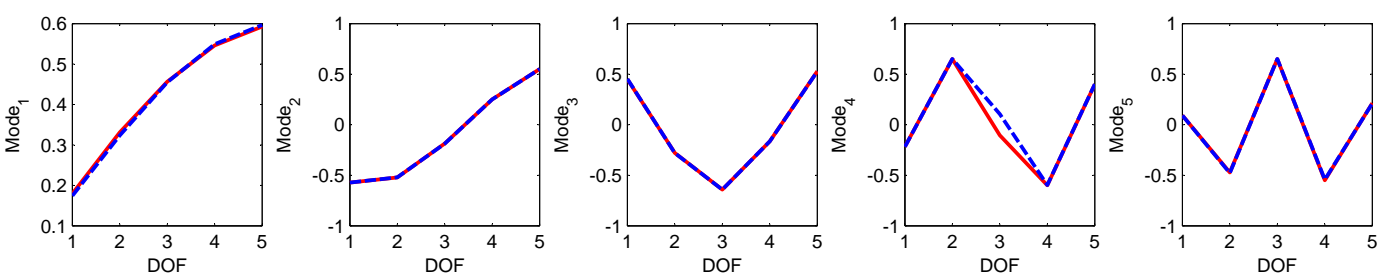

Figure 23. Calculated linear normal modes ( - red) and smooth orthogonal modes (--blue) of the mass spring system. Except for the $4^{\text {th }}$ smooth mode, the modes approximate the LNM almost exactly.

\subsubsection{Approximation of linear normal modes in a continuous beam}

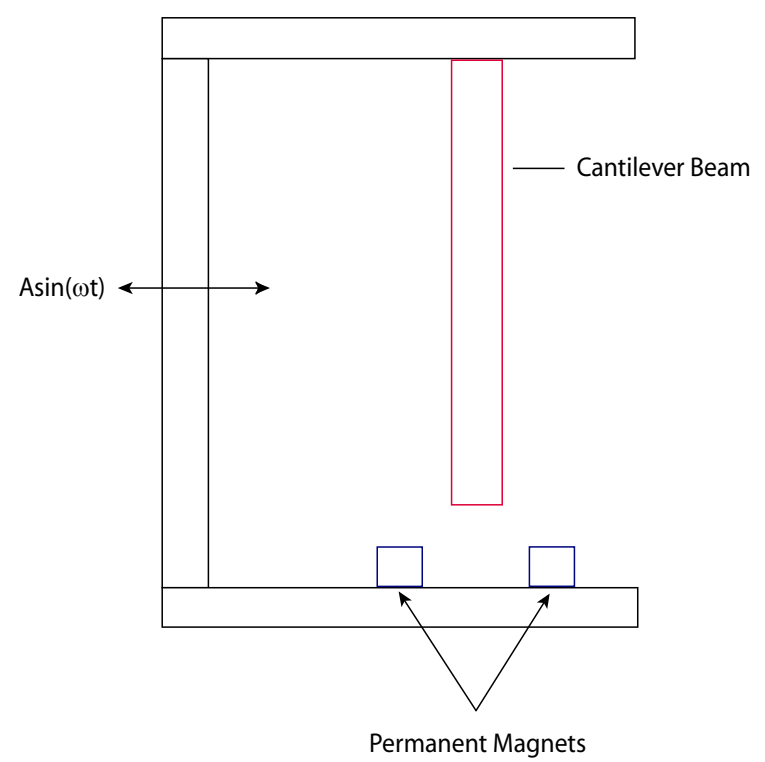

Figure 24. Schematic diagram showing Moon's beam. The nonlinear form is realized by the two permeant magnets. The frame is driven by shacker at a certain frequency.

For the continuous case, we will use Moon's beam [80], Fig. 24 to demonstrate SOD's ability to extract LNMs of a continuous beam. Here, a cantilever beam is 
suspended from a frame that is driven by a shaker. In addition, a nonlinear force is realized by two permanent magnets at an equal distance apart. The beam was modeled and simulated using the finite element method in Matlab. The $2^{\text {nd }}$ order equation of motion representing our system is,

$$
M \ddot{X}+K X=F(X, \dot{X}, t)
$$

where $M$ is the global mass matrix, $K$ is the global stiffness matrix, and $F$ is the forcing term.

The beam is discritized into seven beam elements. The emphasis on this application was not to develop a refined model to obtain a precise time series at each node, but to obtain sufficient data at each node that captures the dynamics of the system response. A more refined mesh would produce more accurate results. Using Euler-Bernoulli elements the local mass and stiffness matrices are calculated for each element. The local Euler-Bernoulli stiffness matrix $K_{i j} \in \mathbb{R}^{4 \times 4}$ matrix

$$
K_{i j}=\frac{E I}{L^{3}}\left|\begin{array}{cccc}
12 & 6 L & -12 & 6 L \\
6 L & 4 L^{2} & -6 L & 2 L^{2} \\
-12 & -6 L & 12 & -6 L \\
-6 L & 2 L^{2} & -6 L & 4 L^{2}
\end{array}\right|
$$

The local mass matrix $M_{i j} \in \mathbb{R}^{4 \times 4}$ matrix

$$
M_{i j}=\frac{\rho A L}{420}\left|\begin{array}{cccc}
156 & 22 L & 54 & -13 L \\
22 L & 4 L^{2} & 13 L & -3 L^{2} \\
54 & 13 L & 156 & -22 L \\
-13 L & -3 L^{2} & -22 L & 4 L^{2}
\end{array}\right|
$$

where $E$ is Young's modulus, $A$ is the area, $L$ is the length of the element, $I$ is the moment of inertia, and $\rho$ is mass density. The material properties for the beam simulation are listed below.

$\mathrm{E}=210 \mathrm{E} 9$ Youngs modulus $\left(\frac{N}{m}\right)$ 
$\mathrm{b}=0.015$ Beam width $(\mathrm{m})$

$\mathrm{h}=0.001$ Beam height $(\mathrm{m})$

$\mathrm{I}=\frac{1}{12 \mathrm{bh}^{3}}$ Moment of Inertia (assume rectangular section)

$\mathrm{A}=\mathrm{bh}$ Beam area $\left(m^{2}\right)$

$\mathrm{L}=0.26$ Length $(\mathrm{m})$

$\rho=7850$ Mass density $\left(\frac{\mathrm{kg}}{\mathrm{m}^{3}}\right)$

The local matrices are assembled into global mass and stiffness matrices while ensuring nodal connectivity. Once the global matrices are constructed, boundary conditions are applied to the global matrices. Since we have a cantilever beam, the first two degrees of freedom on the first node will be fixed. This node is on the element which is theoretically attached to the base. In order to do so, the first two rows and columns will be zeroed out and a one is placed on the diagonal.

The forcing term in Eq. (74) can be expressed as the sum of the force corresponding to the magnetic force $F_{n}(X)$ and the harmonic based excitation force $F_{t}(t)$

$$
F(X, \dot{X}, t)=F_{t}(t)+F_{n}(X)
$$

where

$$
F_{n}^{15}(X)=59.4 X_{7}-36580 X_{7}^{3}=59.4 X_{0,15}-36580 X_{0,15}^{3}
$$

and $X_{7}$ is the relative displacement of node 7 .

The initial conditions are chosen such that all the LNMs are excited. This is achieved by adding scaled normalized LNMs to the equilibrium position. Calculating the LNMs can be ordered into a three step process: (1) find the equilibrium position $(\ddot{X}=\dot{X}=0)$, (2) linearize the nonlinear system about the equilibrium 
position, and (3) calculate the linear normal modes of the linearized system. The matrix composed of LNMs is,

$$
\Phi=\left[\begin{array}{cccccccccccc}
.007 & .207 & .338 & .423 & .455 & -.423 & -.303 & -.333 & -.082 & .108 & .249 & .308 \\
.022 & .434 & .474 & .225 & -.144 & .401 & .358 & .186 & -.256 & -.459 & -.328 & .017 \\
.046 & .531 & .134 & -.398 & -.335 & -.192 & -.395 & -.054 & .449 & .241 & -.318 & -.372 \\
.090 & .469 & -.341 & -.263 & .413 & -.109 & .401 & -.082 & -.446 & .245 & .310 & -.359 \\
.174 & .266 & -.473 & .373 & .006 & .358 & -.378 & .215 & .243 & -.448 & .301 & .078 \\
.338 & -.018 & -.125 & .296 & -.413 & -.428 & .317 & -.308 & .034 & .191 & -.379 & .506 \\
.918 & -.439 & .534 & -.560 & .563 & .545 & -.466 & .838 & -.682 & .648 & -.629 & .610
\end{array}\right] .
$$

The equilibrium position of the beam is shown in Fig. 25. The beam is sim-

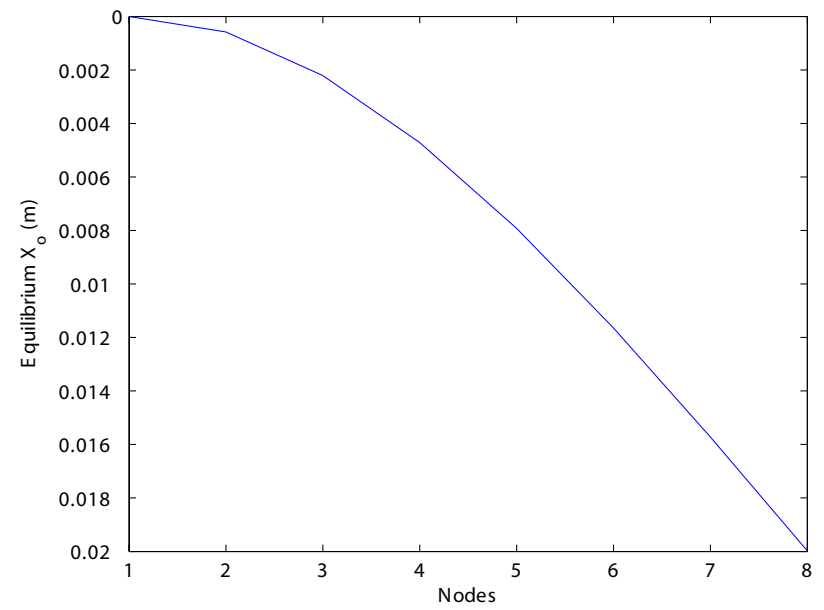

Figure 25. Equilibrium position of beam. The equilibrium is found by setting $\ddot{X}=\dot{X}=0$.

ulated and the position and velocity time series generated at each node are arranged in the ensemble matrices for $\mathrm{SOD}$. The matrices are $Y, V \in \mathbb{R}^{7000 \times 8}$ were constructed and their respective covariance matrices were calculated. Again, this served as the input to the SOD equation, Eq. (43). The modes are estimated by the smooth orthogonal modes which yield,

$$
\psi=\left[\begin{array}{cccccccccccc}
.006 & .206 & .345 & .423 & .453 & -.417 & -.302 & -.334 & -.086 & .108 & .250 & .306 \\
.018 & .432 & .473 & .225 & -.151 & .404 & .361 & .190 & -.254 & -.458 & -.326 & .018 \\
.042 & .533 & .132 & -.397 & -.339 & -.196 & -.391 & -.057 & .451 & .240 & -.319 & -.372 \\
.088 & .471 & -.334 & -.262 & .419 & -.109 & .401 & -.080 & -.448 & .247 & .311 & -.359 \\
.173 & .269 & -.470 & .374 & .011 & .359 & -.376 & .215 & .246 & -.446 & .302 & .077 \\
.338 & -.016 & -.128 & .297 & -.411 & -.428 & .318 & -.307 & .031 & .191 & -.381 & .510 \\
.919 & -.436 & .538 & -.561 & .556 & .546 & -.469 & .837 & -.678 & .650 & -.627 & .608
\end{array}\right] .
$$


In Fig. 26, the calculated LNMs ( - red) of the linearized system and the smooth orthogonal modes (-- blue) are compared. The corresponding values are also listed since the plots are very close. The similarity in the results in both the table values and plots indicate the ability of SOD to extract the LNMs.
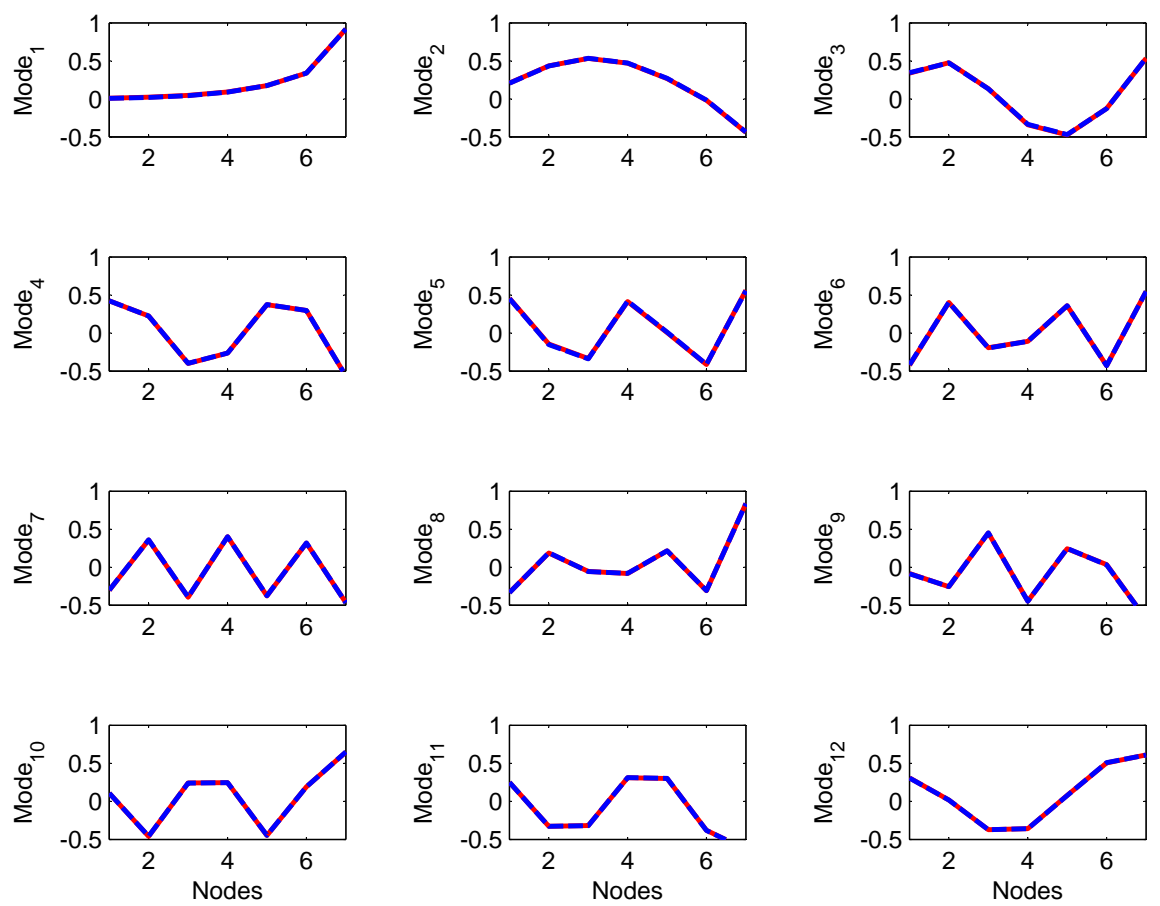

Figure 26. Calculated linear normal modes of the linearized system (-red) and smooth orthogonal modes (--blue) of the continuous beam. The smooth modes almost exactly extract the LNM. 


\section{CHAPTER 4}

\section{Numerical and Experimental Protocols}

In this section, two movement kinematic experiments in which subjects perform a repetitive task and a molecular dynamics simulation of a cyclic pentapentide, will be presented. A data analysis section will follow the description of each experiment and simulation. In the first case, subjects pushed a weighted handle back and forth continuously until volitional exhaustion. The handle was placed at the sternum height (low) and shoulder height (high). Movement kinematics

and electromyography (EMG) were recorded for each subject. In the second case, U.S Army soldiers walked continuously on a treadmill with a 40-kg pack on their back for two hours. Movement kinematics, EMG, and breath-by-breath oxygen consumption $\left(\dot{V} \mathrm{O}_{2}\right)$ were recorded. Lastly, a five residue cyclic pentapeptide was simulated using the custom software package, GROMACS. Position and velocities of each atom in the backbone chain of the peptide were recorded.

\subsection{Subjects Performing a Sawing Motion 4.1.1 Subjects}

The work in this section was conducted at the University of Texas at Austin's Nonlinear Biodynamics Laboratory by Professor Jonathan Dingwell and Dr. Deanna Gates. Ten healthy right-handed subjects (six male, four female) participated in this work (Table 1). Their mean (STD) age, weight, and height were 27 (2) yr, $72.4(18.2) \mathrm{kg}$, and $1.73(0.10) \mathrm{m}$, respectively. Prior to the experiment, all participants signed institutionally approved consent forms and were screened to ensure that no subject had a history of medications, surgeries, injuries, or illnesses that might have affected their upper extremity joint movements. 
Table 1. Basic characteristics for each sawing subject.

\begin{tabular}{cccccc}
\hline $\begin{array}{c}\text { Subject } \\
\text { Number }\end{array}$ & $\begin{array}{c}\text { Gender } \\
\mathrm{M} / \mathrm{F}\end{array}$ & $\begin{array}{c}\text { Age } \\
(\mathrm{yrs})\end{array}$ & $\begin{array}{c}\text { Body Mass } \\
(\mathrm{kg})\end{array}$ & $\begin{array}{c}\text { Weight Applied } \\
(\mathrm{kg})\end{array}$ & $\begin{array}{c}\text { Metronome } \\
(\mathrm{bpm})\end{array}$ \\
\hline 1 & $\mathrm{~F}$ & 28 & 55.0 & 4.54 & 133 \\
2 & $\mathrm{M}$ & 26 & 71.2 & 7.37 & 128 \\
3 & $\mathrm{M}$ & 32 & 95.5 & 9.07 & 123 \\
4 & $\mathrm{M}$ & 29 & 110.5 & 6.80 & 123 \\
5 & $\mathrm{~F}$ & 30 & 62.5 & 3.40 & 133 \\
6 & $\mathrm{M}$ & 26 & 61.5 & 5.67 & 131 \\
7 & $\mathrm{~F}$ & 29 & 51.5 & 3.40 & 129 \\
8 & $\mathrm{M}$ & 28 & 70.0 & 5.67 & 126 \\
9 & $\mathrm{~F}$ & 26 & 70.0 & 5.67 & 131 \\
10 & $\mathrm{M}$ & 25 & 76.2 & 6.01 & 126 \\
\hline Mean \pm SD: & - & $27 \pm 2$ & $72.4 \pm 18.2$ & $5.67 \pm 1.74$ & $128 \pm 4$ \\
\hline \hline
\end{tabular}

\subsubsection{Experimental Protocol}

Subjects performed two repetitive tasks at two different heights approximately one week apart. In each case, the subjects pushed a weighted handle back and forth in a sawing motion. During the first visit, the handle was placed in a low position such that the handle aligned with the subject's sternum. In the later visit, the handle was placed in a high position such that the handle was at the subject's shoulder height.

During each experiment, subjects were seated in a chair and made a bidirectional horizontal movement in the anterior-posterior direction with their right arm. The subjects were placed so their knee angle was at $90^{\circ}$ and their movements were restricted by a five-point harness (Corbeau, Sandy, UT, USA). The handle was attached to a metal platform that slides on a low frictional track. Inertial resistance was applied by adding weights on the handle. Therefore, a resisting load always opposed the direction of movement so that the arm extensors and flexors were equally worked, Fig. 27. Subjects performed this task continuously until voluntary exhaustion (refer to [81]).

The amount of weight that was placed on the handle was $15 \%$ of their max- 


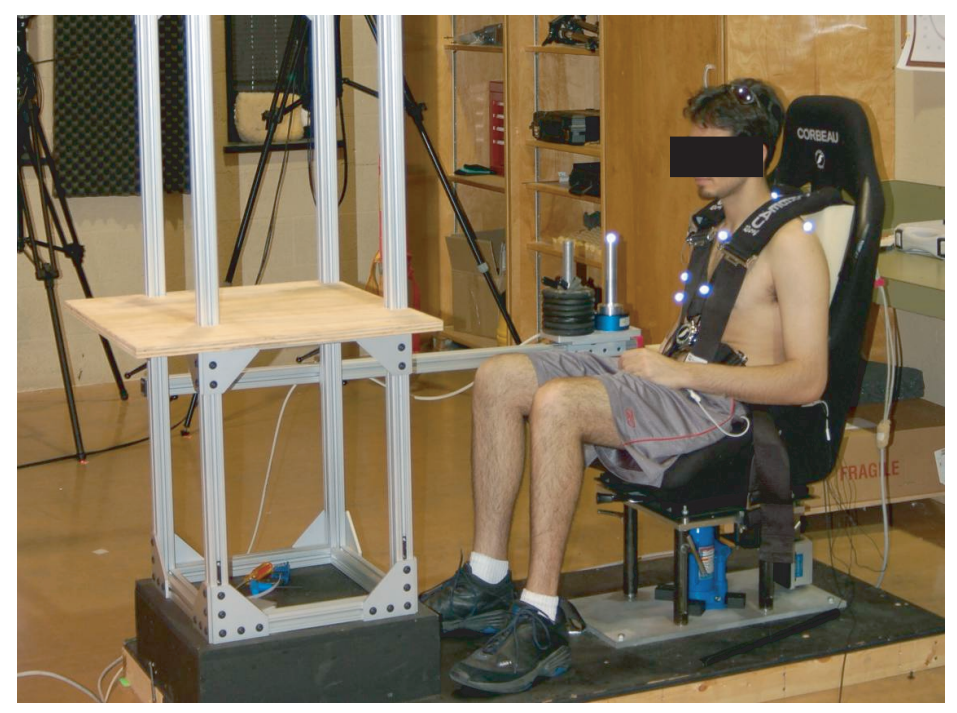

Figure 27. Experimental setup for subjects sawing. Picture courtesy of Dr. Deanna Gates and Professor Jonathan Dingwell.

imum pushing/pulling force. This percentage was chosen so complete fatigue in the subjects performing the low task could be achieved in approximately 15-20 minutes. Each subject pushed and pulled a handle with a Baseline ${ }^{\circledR}$ dynamometer with maximal effort three times for five seconds each time with at least one minute of rest in between each attempt. The maximum weight was defined by the subject's isometric pushing/pulling force for each attempt.

To ensure that the task was dynamically equivalent from subject to subject, participants were instructed to synchronize their movements to a metronome $(\approx 1 \mathrm{~Hz})$. Each subject warmed up for approximately thirty seconds with the metronome to ensure that they were comfortable with the sawing motion. The frequency value was equal to approximately two beats per movement cycle (one complete push and pull) and was calculated using,

$$
f_{n}=\frac{1}{2 \pi} \sqrt{\frac{m g r}{I_{o}}},
$$

where the arm segment was treated as a rigid body, $m$ is the mass of the limb segment, $I_{o}$ is the moment of inertia of the limb segment about the axis of rotation, 
and $r$ is the distance from the axis of rotation to the center of mass of the limb segment. Values for $m, I_{o}$, and $r$ where estimated from standard anthropometric tables (refer to [82]).

To capture movement kinematics, nineteen reflective markers were placed on the arm and trunk to define the movements of four body segments. Markers were placed on the trunk at the right and left acromion processes and sternal notch. Clusters of four markers were placed on the upper and lower arms to define the segments. The hand was defined by four markers at the ulnar epicondyle of the wrist and third and fifth metacarpal-phalangeal (MCP) joints. Additional markers were placed on the medial and lateral humeral epicondyle for a static calibration trial. One additional marker was placed on the top of the handle to define the beginning and end of each cycle. The three dimensional movements of these markers were recorded continuously during all trials at $60 \mathrm{~Hz}$ using an 8-camera Vicon-612 motion analysis system (Oxford Metrics, Oxford, UK).

To capture EMG, nine preamplified EMG surface electrodes (Delsys Inc., Boston, MA) were attached to the right arm and torso in the flexor carpi radialis (FCR), extensor carpi radialis longus (ECR), pectoralis major, biceps femoris, triceps (lateral head), deltoid (middle, posterior, and lateral), middle trapezius to record activity, see Fig. 28 for gross anatomy of the upper body. Electrodes were positioned over each muscle according to accepted recommendations [83]. EMG signals were recorded at $1080 \mathrm{~Hz}$ using a Delsys Bagnoli-8 system integrated with the Vicon-612 system, see Figs. 29-30 for plots of recorded raw EMG signals in the high sawing experiment and low sawing experiment, respectively.

\subsubsection{Data Analysis}

Kinematic marker data was filtered using a fifth order Butterworth filter with a cutoff frequency of $15 \mathrm{~Hz}$. Segment coordinate systems were calculated based on 


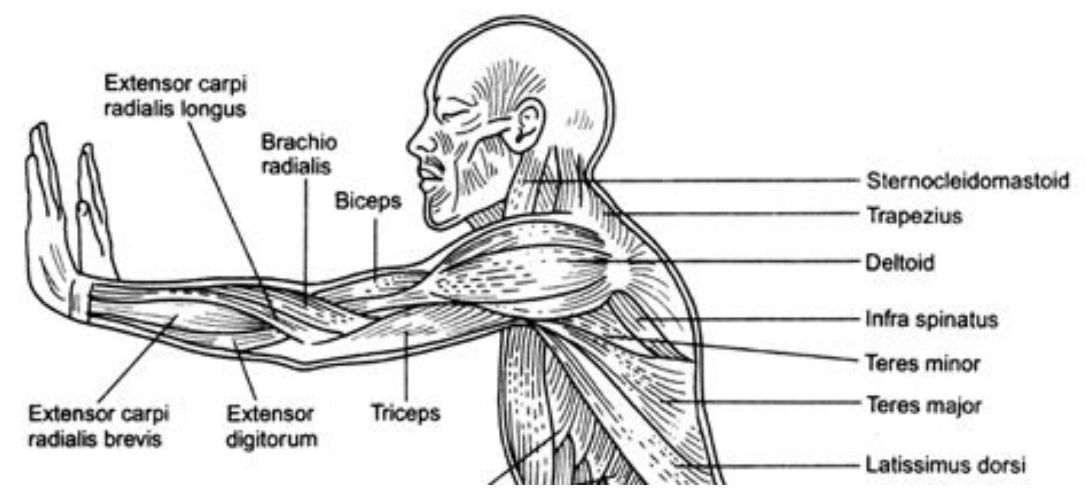

Figure 28. Gross anatomy figure of the upper body showing where the muscles are located.

the marker positions with a least-squares algorithm [84]. The joint centers at each instant in time were calculated based on the position of the joint markers during the static trial [85]. Local coordinate systems were defined using the International Society of Biomechanics'(ISB) recommendations for the humerus and forearm [86] and a modified coordinate system for the trunk [87] and wrist [88]. The three dimensional movements of the right arm were determined using Euler rotations in accordance with ISB recommendations [86]. Three sets of angles were measured from the shoulder (humeral plane, elevation, rotation), elbow (flexion/extension, pronation/supination, and carrying angle[essentially zero]), and wrist (flexion/extension, ulnar/radial deviation, pronation/supination).

The raw EMG is first filtered through a bandpass filter with cutoff frequencies $\omega_{1}=20 \mathrm{~Hz}$ and $\omega_{2}=450 \mathrm{~Hz}$ to eliminate DC offset and attenuate high frequency noise. The effects of muscle motor unit firing are reflected in the spectrum of EMG frequencies of $15 \mathrm{~Hz}$ to $25 \mathrm{~Hz}$. It is recommended in [57] to set the lowfrequency cutoff at $20 \mathrm{~Hz}$ to maintain signal stability, improve stationarity, and eliminate motion artifacts. The EMG time series is then filtered through a notch filter with cutoff frequencies $\omega_{1}=59 \mathrm{~Hz}$ and $\omega_{2}=61 \mathrm{~Hz}$ to attenuate any $60 \mathrm{~Hz}$ $\mathrm{AC}$ interference. 

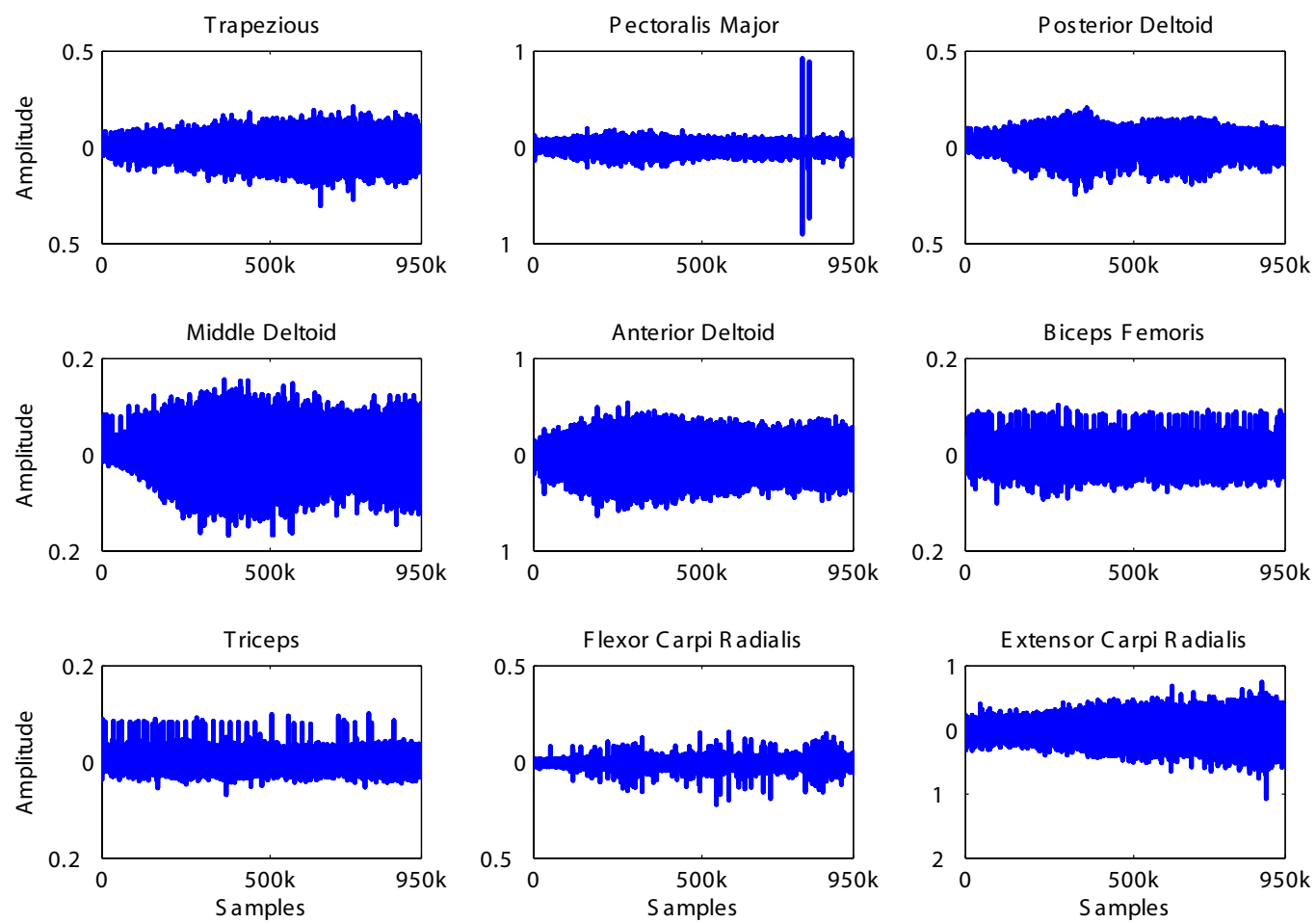

Figure 29. Recorded raw Electromyography (EMG) time series for subject 6 for the high sawing experiment

It has been shown that as a muscle fatigues, the bandwidth of the EMG spectrum shifts towards lower frequencies [56]. To this end, fatigued muscles should show a decrease in the mean frequency of the power spectrum. The mean power frequencies (MNF) of the EMG power spectrum were used to indicate muscle fatigue [35]. The MNF can be calculated as follows,

$$
\mathrm{MNF}=\frac{\int_{0}^{f} f S_{m}(f) d f}{\int_{0}^{f} S_{m}(f) d f}
$$

using numerical integration to evaluate the integrals where $S_{m}(f)$ is the power density spectrum of the EMG signal.

EMG goes through cycles of accumulation and relief. The entire time series is considered nonstationary over these regions of accumulation and relief [83]. However, over each stroke the time series can be considered stationary. Time points defining the beginning, middle, and end of each cycle, as determined from the 

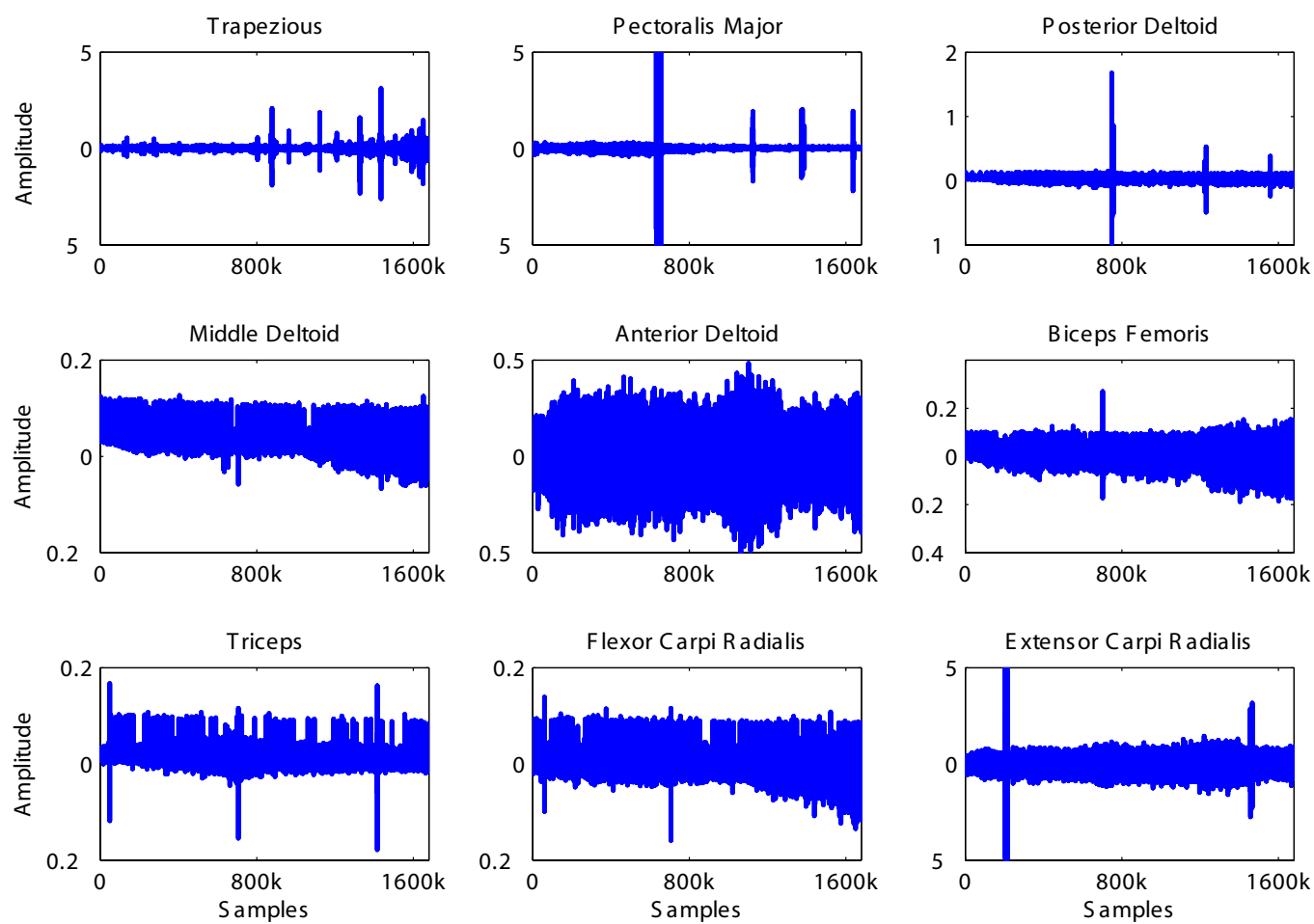

Figure 30. Recorded raw Electromyography (EMG) time series for subject 6 for the low sawing experiment

marker data, were used to split each EMG signal into the push stroke and pull stroke. The MNF for each stroke (either push or pull) were computed from the power spectrum of the signal using Welch's method. Welch's method estimates the power spectral density by taking the average of the calculated periodograms over slip or overlapping segments of the input time series. The MNF for each complete movement cycle (push plus pull) were calculated as the average of the MNFs for the push and pull strokes [55], Figs. 31-32 for plots of the high sawing experiment and low sawing experiment, respectively.

The resulting truncated EMG time series can be considered stationary over each stroke so the data can be analyzed. A moving average filter is applied to this truncated time series to produce the final time series of interest. Figs. 33-34 shows the resulting EMG trends that will later be compared against the extracted 
smooth coordinates from the PSW-based feature space for the high and low sawing experiment, respectively.
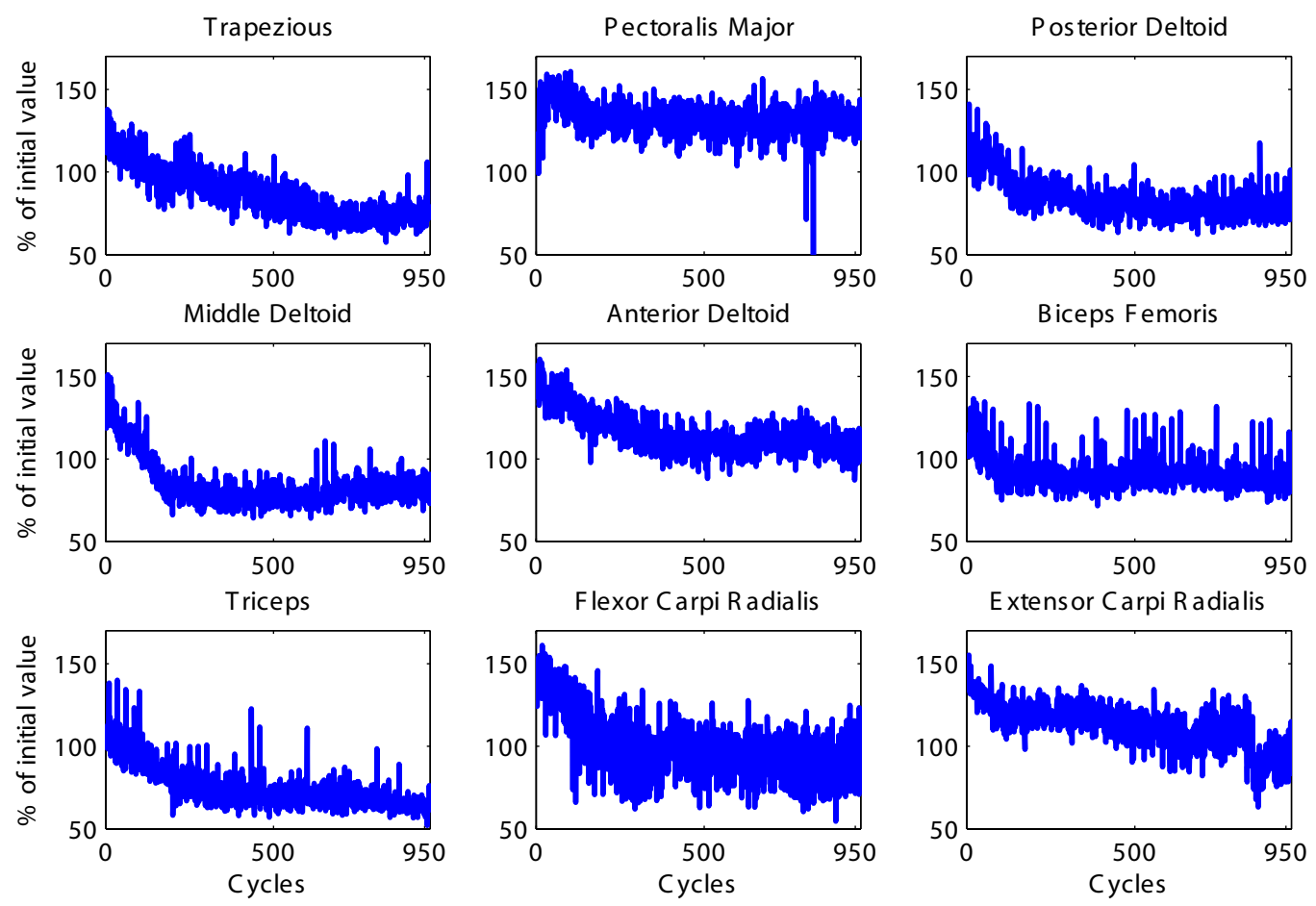

Figure 31. Mean power frequencies ( MNF ) calculated over individual cycles of EMG data of subject 6 for the high sawing experiment 

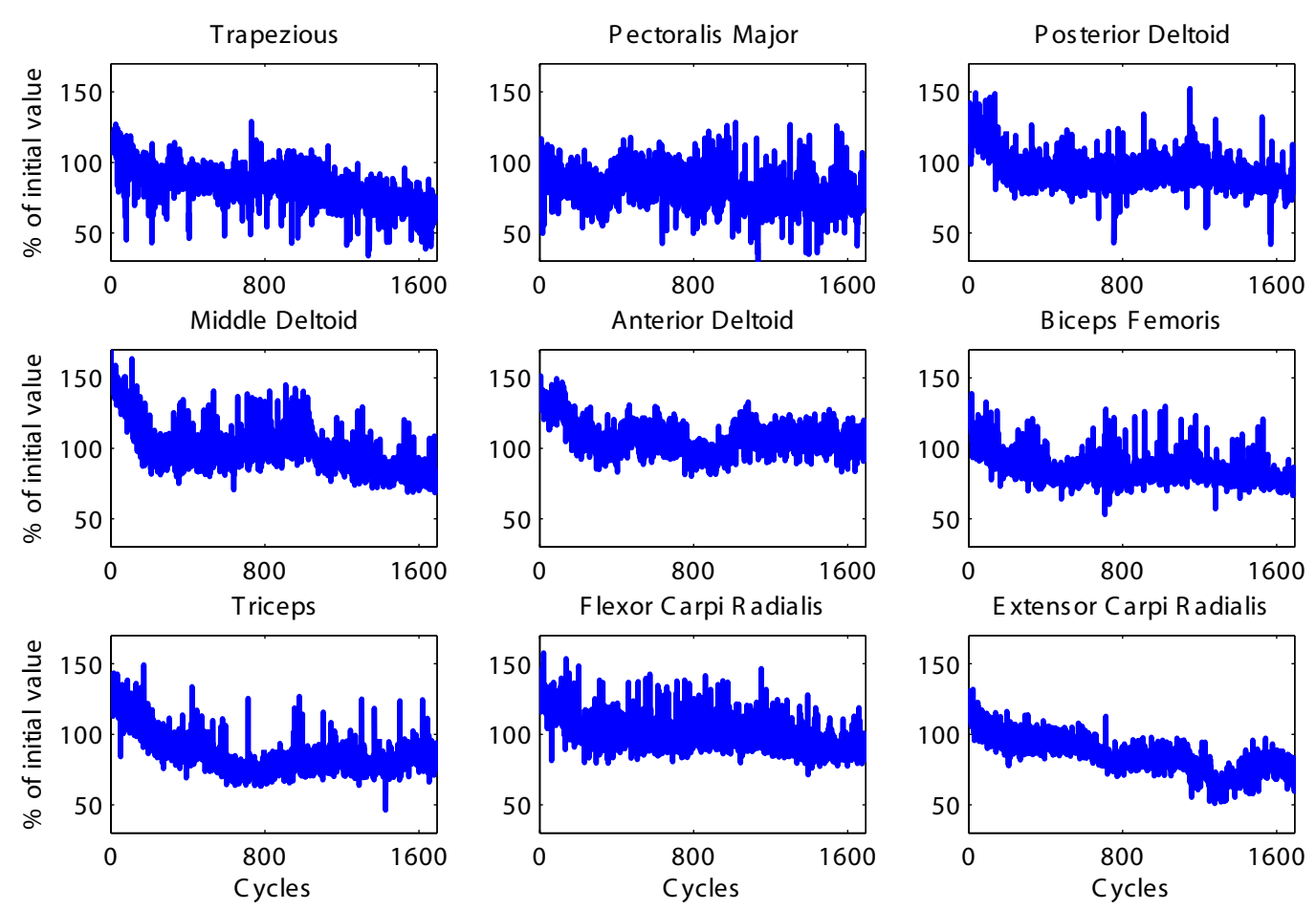

Figure 32. Mean power frequencies ( MNF ) calculated over individual cycles of EMG data of subject 6 for the low sawing experiment
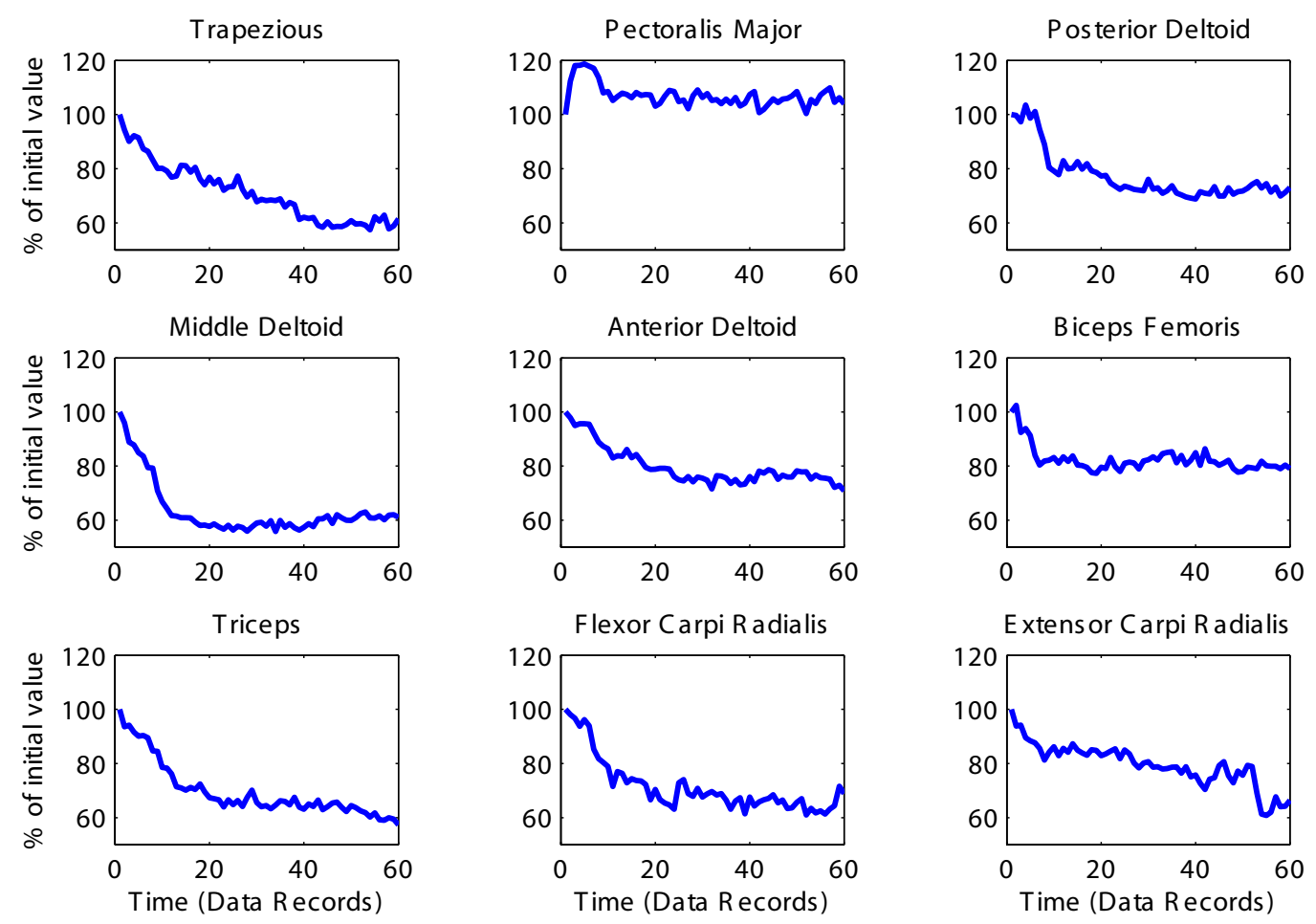

Figure 33. Averaged MNF for subject 6 for the high sawing experiment 

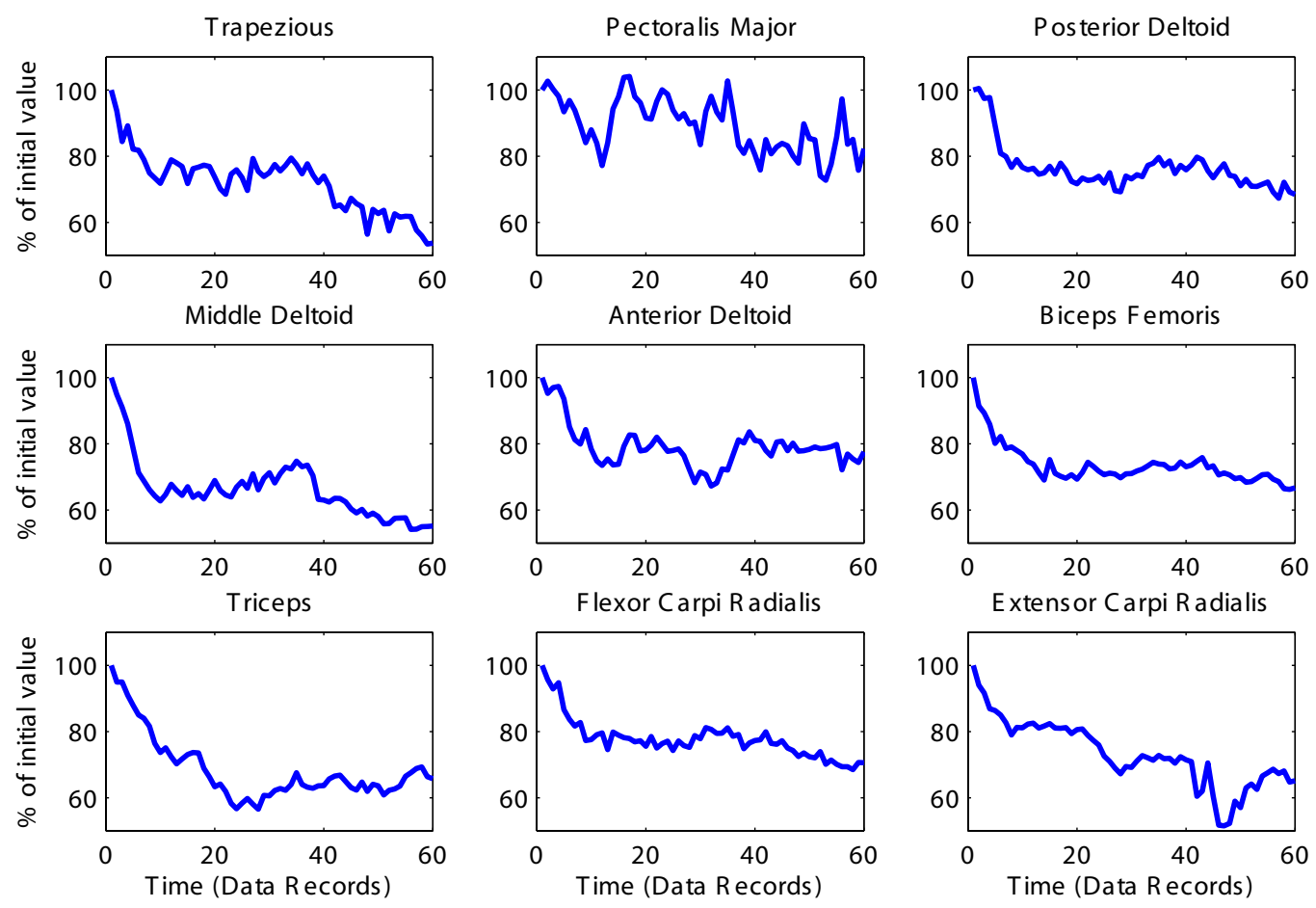

Figure 34. Averaged MNF for subject 6 for the low sawing experiment 
Table 2. Basic characteristics for each U.S Army soldier.

\begin{tabular}{ccccc}
\hline \hline $\begin{array}{c}\text { Subject } \\
\text { Number }\end{array}$ & $\begin{array}{c}\text { Gender } \\
\text { M/F }\end{array}$ & $\begin{array}{c}\text { Age } \\
(\mathrm{yrs})\end{array}$ & $\begin{array}{c}\text { Body Mass } \\
(\mathrm{kg})\end{array}$ & $\begin{array}{c}\text { Height } \\
(\mathrm{m})\end{array}$ \\
\hline 1 & $\mathrm{M}$ & 22 & 76 & 1.8 \\
2 & $\mathrm{M}$ & 28 & 82 & 1.7 \\
3 & $\mathrm{M}$ & 20 & 74 & 1.7 \\
\hline Mean \pm SD: & - & $23.3 \pm 4.2$ & $77.3 \pm 4.2$ & $1.73 \pm 0.06$ \\
\hline \hline
\end{tabular}

\subsection{Load Carrying U.S Army Soldiers 4.2.1 Subjects}

The work in this section was conducted at Natick System Soldiers Center in Natick, Massachusetts by Dr. Jeffrey Schiffman and collaborators. Three healthy U.S Army soldiers participated in this work (Table 2). Their mean (STD) age, weight, and height were $23.3(4.2) \mathrm{yr}, 77.3(4.2) \mathrm{kg}$, and $1.73(0.06) \mathrm{m}$, respectively. Prior to the experiment, informed consent was obtained and the study was approved by the Institutional Review Board and conducted in accordance with Federal Policy for the Protection of Human Subjects, Department of Defense, 32 CFR Part 219.

\subsubsection{Experimental Protocol}

U.S Army soldiers walked continuously on a custom built treadmill (AMTI, Watertown, MA) at $1.33 \mathrm{~m} / \mathrm{s}$ while carrying a 40-kg pack. The 40-kg pack consisted of clothing, protective equipment, and a rucksack. The custom built treadmill is comprised of two synchronized treadmills on a single platform and is capable of measuring ground reaction forces (kinetics) in three planes. Each treadmill is mounted on its own force plate.

Throughout the entire two hour bout, kinetics, kinematics, EMG, and breathby-breath oxygen consumption $\left(\dot{V} O_{2}\right)$ were measured. Three dimensional motion kinematics were recorded by motion capture unit cameras (Qualisys Medical AB, Gothenburg, Sweden). To capture movement kinematics, sixteen retro-reflective 
markers were placed on the skin and clothing of the soldiers. Three dimensional time histories were recorded by eight surrounding cameras. All markers were placed on the right lateral toe, lateral ankle, heel, lateral knee, greater trochanter, shoulder, upper and lower sternum; four marker clusters were placed at the right shank and thigh, see Fig. 35 .

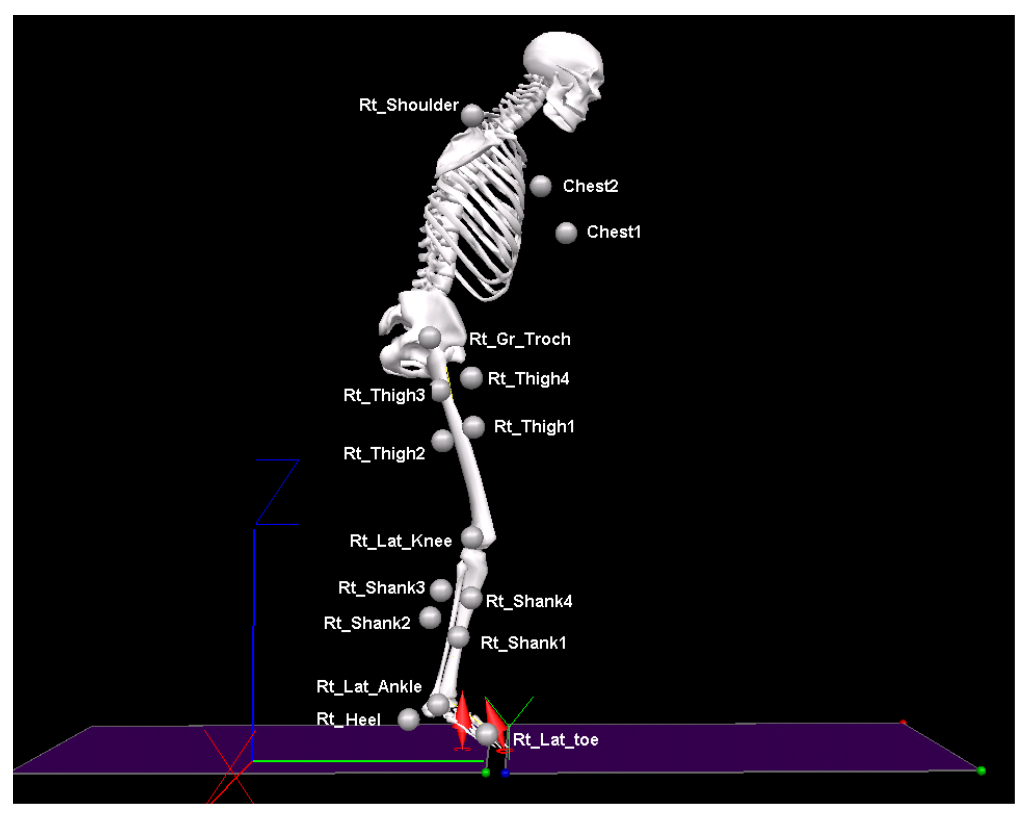

Figure 35. Marker location for U.S Army soldiers. Picture courtesy of Dr. Jeffrey Schiffman

Gait kinematic data were collected at $60 \mathrm{~Hz}$ in sequential five-minute bins throughout the two hour walking period. Approximately thirty seconds existed between the sequential bin collections. The Visual 3-D software package (C-motion Inc., Rockport, MD) was used to process the data files into movement kinematic time series for the trunk lean, ankle, knee, and hip angle.

EMG was captured by placing ten preamplified bipolar surface electrodes (Delsys, Boston, MA) placed bilaterally on selected leg and trunk muscles. EMG was recorded using a Delsys Bagnoli-8 system at $600 \mathrm{~Hz}$ from the bilateral tibialis anterior, gastrocnemius, rectus femoris, vastus medialis, and the vastus lateralis 


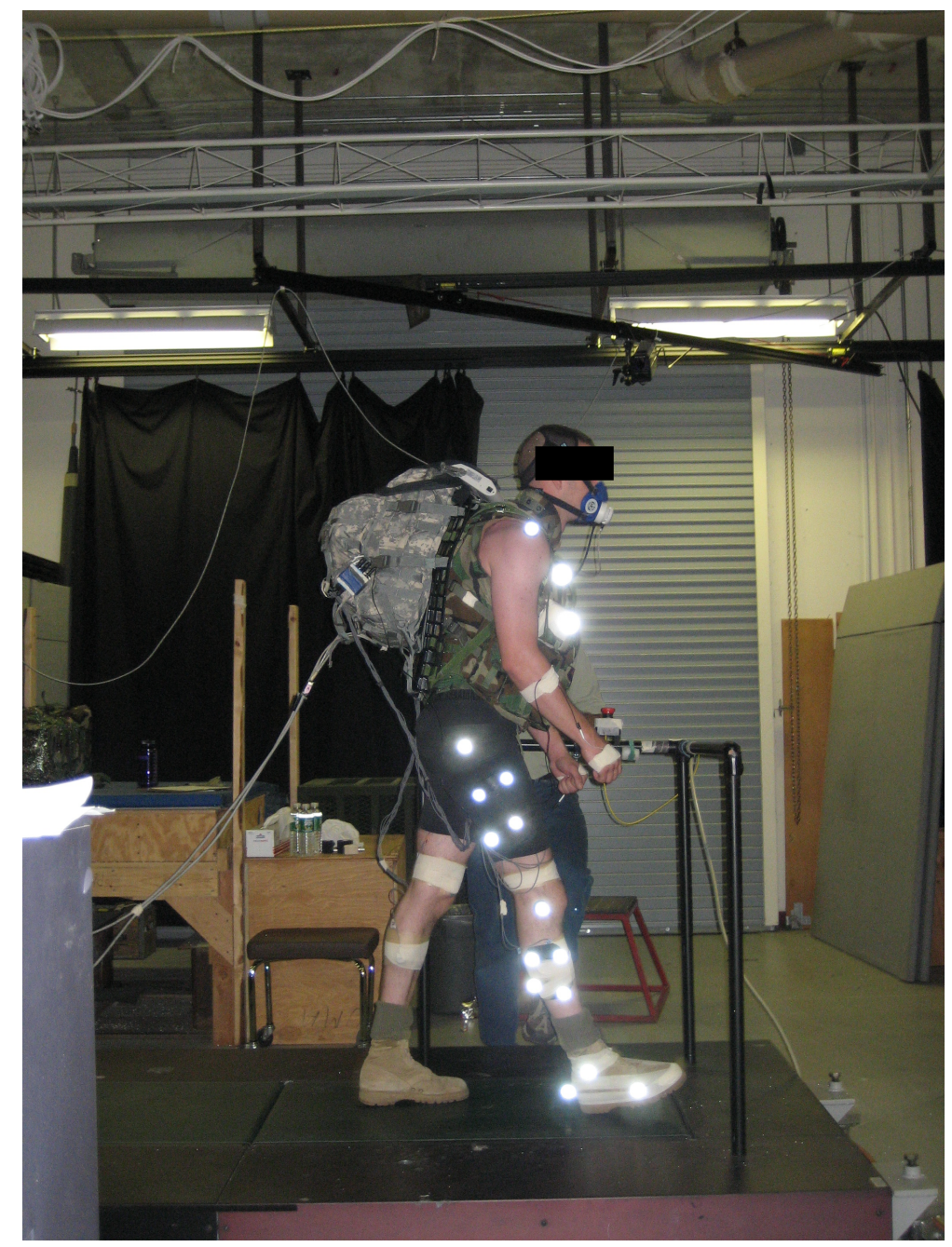

Figure 36. Experimental setup for U.S Army soldiers. Picture courtesy of Dr. Jeffrey Schiffman

muscles, see Fig. 28 for gross anatomy of the lower body. Fig. 38 depicts the plots of recorded EMG signals for each muscle. Breath-by-breath oxygen consumption $\left(\dot{V} \mathrm{O}_{2}\right)$ is recorded continuously using a ParvoMedics TrueMax 2400 metabolic measurement system with a sampling of 36 breaths/min.

\subsubsection{Data Analysis}

The processing of the data is very similar to the previous section; therefore some repetitive details will be excluded here and the reader is referred to the preceding section. The raw EMG is bandpass filtered with a cutoff frequencies of 


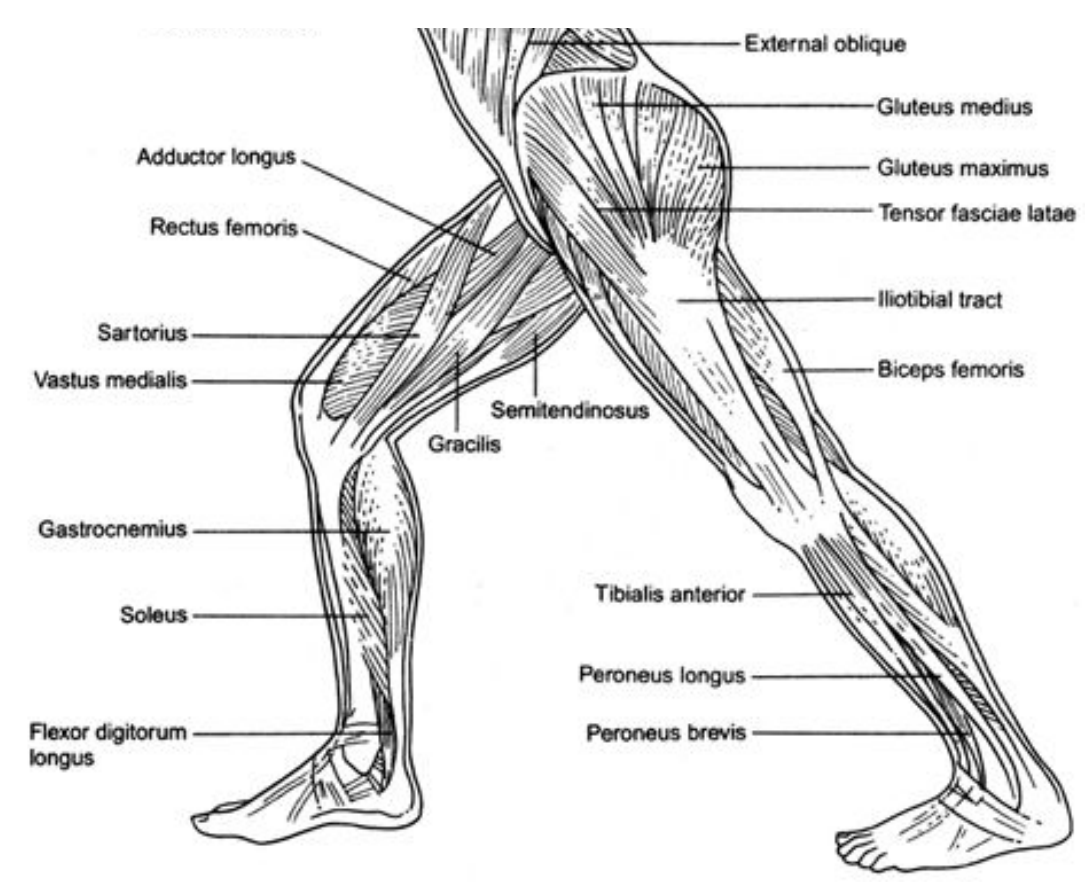

Figure 37. Gross anatomy figure of the lower body showing where the muscles are located.

$\omega_{1}=20 \mathrm{~Hz}$ and $\omega_{2}=200 \mathrm{~Hz}$ and then filtered through a notch filter with cutoff frequencies $\omega_{1}=59 \mathrm{~Hz}$ and $\omega_{2}=61 \mathrm{~Hz}$.

The instantaneous mean frequency was calculated using a continuous wavelet transform (CWT) due to the fact that cycles of accumulation and relief have not been defined [89]. The continuous wavelet transform is a joint time and frequency method that reconstructs a time-frequency representation of a signal. It is analogous to Fourier analysis but includes time scales. The power density function, or 'Scalogram', of the CWT is

$$
\operatorname{SCAL}(\tau, s)=|\mathrm{CWT}(s, \tau)|^{2}
$$

where $s$ represents the scale (frequency band) and $\tau$ is time. The instantaneous mean frequency (IMNF) is calculated by

$$
\operatorname{IMNF}=\frac{\int_{l s}^{h s} s \times \operatorname{SCAL}(s) d s}{\int_{l s}^{h s} \operatorname{SCAL}(s) d s},
$$



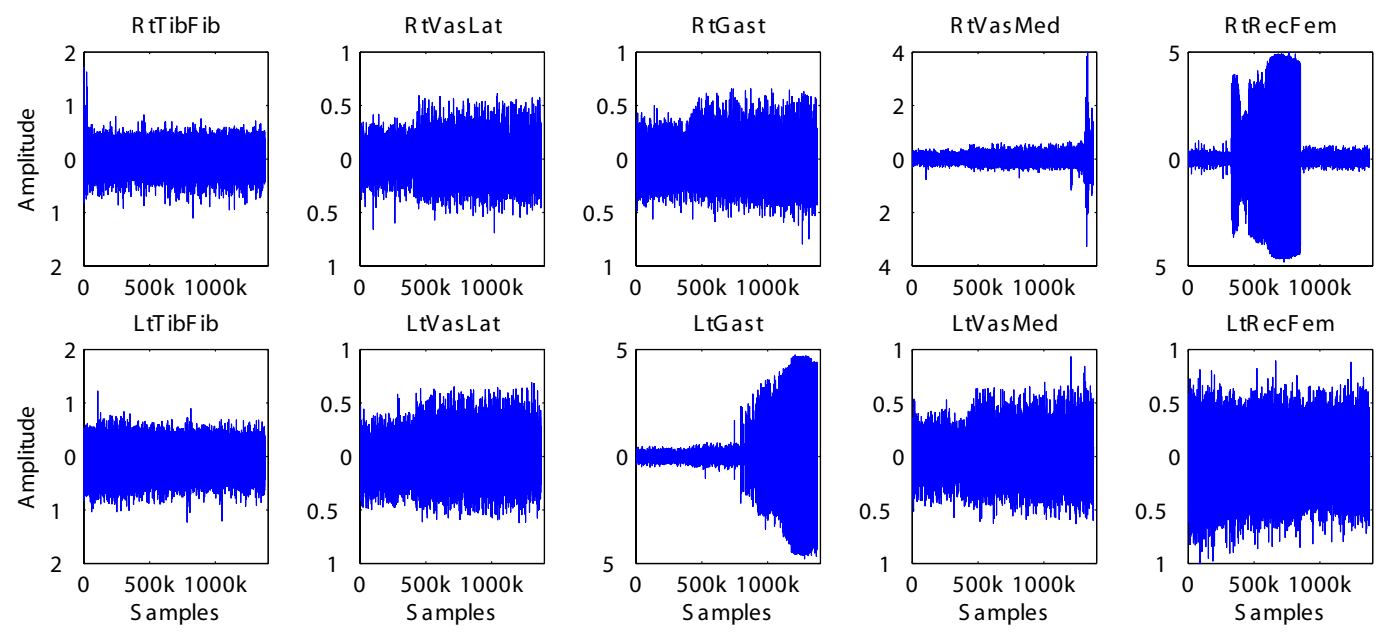

Figure 38. Recorded EMG over the 120 minute march from each muscle for subject 10.

using numerical integration to evaluate the integrals where $l s$ is the lowest scale of interest and $h s$ is the highest scale of interest. A 'debauchies' wavelet (db5) with 1 -scale intervals from 136 is used. The max scale was calculated by $a_{\max }=\frac{5.6 \delta t}{\pi}$ where $\delta t$ is the frequency value of $20 \mathrm{~Hz}$ and the value of 5.6 was determined from other studies [90, 89]. The lowest scale corresponds to $444 \mathrm{~Hz}$, which ensures at least one stride period is contained in the wavelet. The IMNF is then averaged over equal length windows to create a time series with a desired length, see Fig. 39.

$\dot{V} \mathrm{O}_{2}$ data greater than 3 standard deviations from the mean is removed, see Fig. 40 (Left). A convolution-based triangle filter is used to smooth $\dot{V} \mathrm{O}_{2}$ data. First, a triangle function is created by convolving two rectangular functions. Then the $\dot{V} O_{2}$ data is convolved with the triangle function. Data is then averaged over equal length windows to create a time series with a desired length. The raw data shows no discernable trend with 36 breaths/min; therefore, applying the smoothing function will not remove any important slow-time artifacts of the data set. The length of the time series is the same as the length of the time series representing the smooth coordinates extracted from SOD, see Fig. 40 (Right). This allows for 

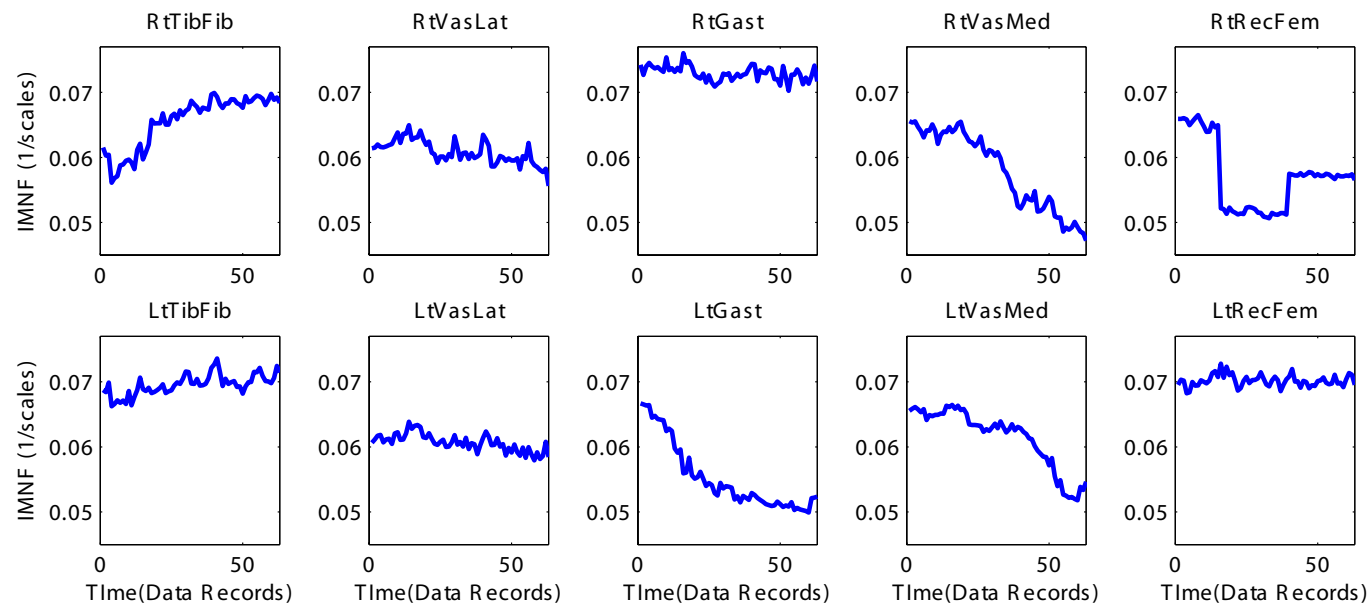

Figure 39. Calculated instantaneous mean frequency for each muscle and then averaged to a truncated time series of 63 points.

comparing the smooth coordinates against recorded $\dot{\mathrm{V}} \mathrm{O}_{2}$.
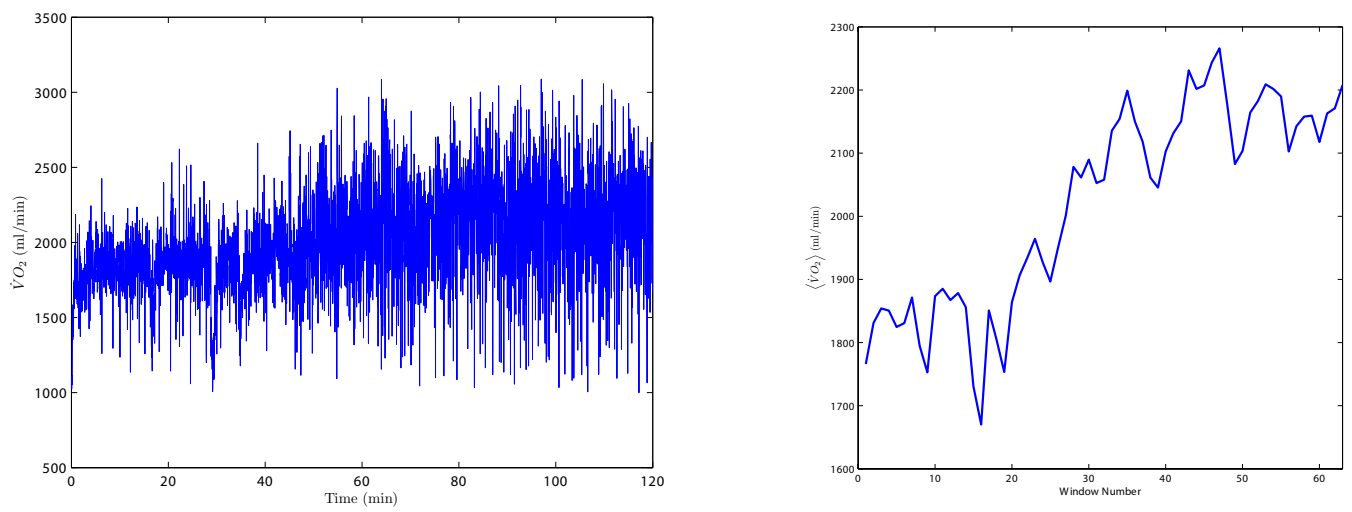

Figure 40. (Left) Recorded $\dot{V} O_{2}$ over the 120 minute length of the experiment. Data greater then three standard deviations from the mean are removed. (Right) Averaged $\left\langle\dot{V} \mathrm{O}_{2}\right\rangle$ over equal length windows after the convolution-based triangle filter is applied to the recorded data. 


\subsection{Molecular Dynamics of Cyclic Pentapeptide 4.3.1 Molecular Simulation}

The work in this section was conducted by Professor Dale Mierke of Dartmouth College. Using the custom software program GROMACS, a five residue cyclic pentapeptide-cyclo(d-Pro1-Ala2-Ala3-Ala4-Ala5) in DMSO (Dimethyl Sulfoxide) solution was numerically simulated. Fig. 41 shows a schematic representation of the peptide. In the figure, each residue is labeled and the blue lines represent nitrogen atoms, red lines represent oxygen atoms, and green lines represent carbon atoms.

The peptide was simulated for $22 \mathrm{~ns}$ with an integration time step of $1 \mathrm{fs}$ with an applied constant pressure of 1 bar. All atoms were treated in the OPLS (optimized potentials for liquid simulation)-AA force field, [91, 92]. To model an explicit solvent environment, the peptide structure is placed in a simulation box where each side of the box has a length of $40 \AA$, which contains typically 487 DMSO molecules. The entire system was energy-minimized using the steepest descent algorithm.

The system was initially simulated with constraints and then the constraints were removed to allow the system to evolve. The constraints were experimental distances derived from REDOR - a high resolution solid NMR experiment - for Ala3N-Ala5C' and Ala3C'-Ala3N. The length of time for the constraints were $2 \mathrm{~ns}$ at a temperature of $300 \mathrm{~K}$ (with 0.02 ps temperature bath coupling) with a force

constant of $500 \frac{\mathrm{kJ}}{\mathrm{mol} \cdot \mathrm{nm}}$ to allow the system to come to equilibrium. The constraints were removed for the remaining $20 \mathrm{~ns}$ and the system was allowed to evolve freely. Position and velocity components were saved at every $0.25 \mathrm{ps}$ for all atoms in the backbone structure.

The nature of this peptide is the motion of the Ala3 residue. To ensure that our simulation is correct, the following figures were compared with the results from 


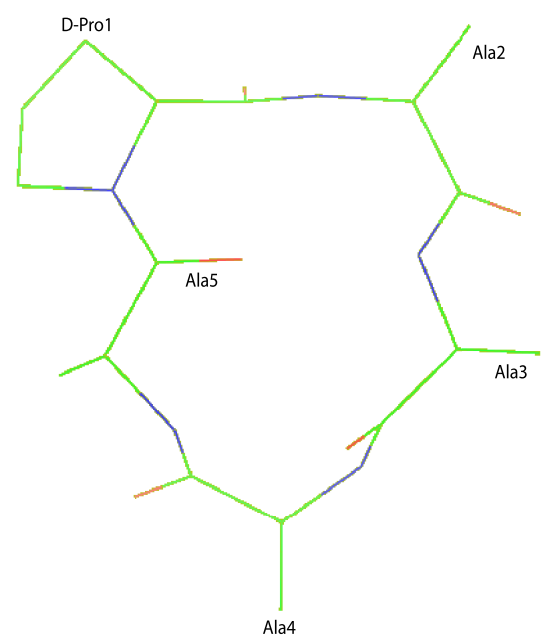

Figure 41. Five residue cyclic pentapeptide-cyclo(d-Pro1-Ala2-Ala3-Ala4-Ala5): (-blue) nitrogen atoms, (-red) oxygen atoms, (-green) carbon atoms.

[93]. Fig. 42 (Left) shows the Ramachandran plot of the $\phi-\psi$ dihedral angles for all residues in the peptide. Each dot corresponds to a specific $\psi-\psi$ angle. High density regions on the plot correspond to low energy configurations of the peptide. Figure 42 (top right) depicts the rmsd (root mean square deviation) plot of the peptide backbone after a least-squares fit to the initial backbone structure. The rmsd plot gives a numerical measure of the difference between two structures and is calculated by

$$
\operatorname{rmsd}=\sqrt{\frac{\sum_{i=1}^{N}\left(r_{i}\left(t_{1}\right)-r_{i}\left(t_{2}\right)\right)^{2}}{N}}
$$

where $N$ is the number of atoms whose positions are being compared and $r_{i}(t)$ is the position of atom $i$ at time $t,[94]$. In the figure, the conformational transitions of the conformational states can be observed from this plot at 5.5, 11, and $13.7 \mathrm{~ns}$. Also, Fig. 42 (Bottom Right) shows the cosine of the angle between the C-O vector of Ala3 and the peptide ring plane defined by three atoms: Ala5 $\mathrm{C} \alpha$, Ala2C $\alpha$, and Ala3C'. This plot agrees with the times of conformational transitions. 

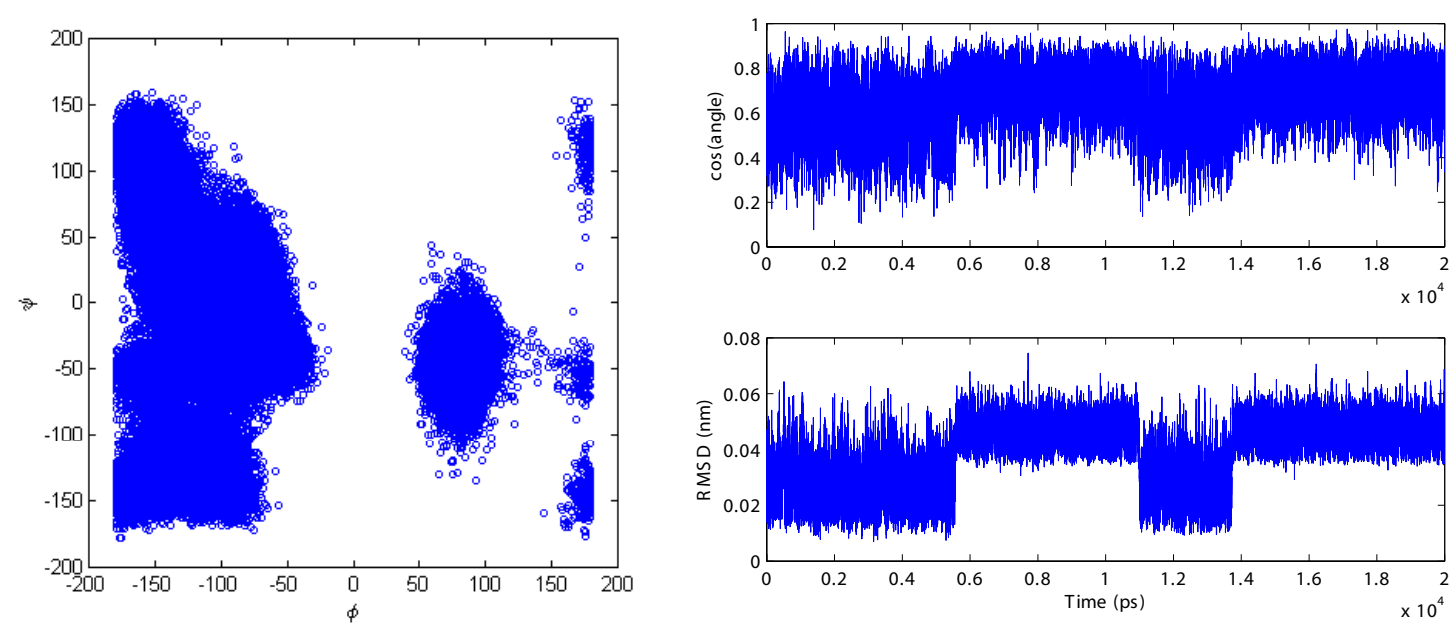

Figure 42. (Left) Ramachandran plot after simulation that shows five different possible conformations of the peptide by the $\psi-\psi$ dihedral angles. (Top Right) An rmsd plot of the peptide backbone after a least-squares fit to the initial backbone structure. (Bottom Right) Cosine of the angle between the $\mathrm{C}-\mathrm{O}$ vector of Ala3 and the peptide ring plane defined by three atoms, Ala5 $\mathrm{C} \alpha$, Ala2 $\mathrm{C} \alpha$, and Ala3C'. Conformational transitions occur at 5.5, 11, and $13.7 \mathrm{~ns}$.

\subsubsection{OPLS Force Field}

The classical computational methods in simulating a molecular structure is through molecular dynamics or Monte Carlo statistical mechanics. The outcome of these methods is controlled by the total energy and is referred to as the force field. The force field used in this simulation is the OPLS-AA.

The OPLS all atom force field was developed by Jorgensen et al. to study the molecular mechanics of organic molecules and peptides [91, 92]. This model considers atoms as spheres and the bonds as springs. By using a spring to describe the bond, we can mathematically account for bond stretching, bending, and twisting. The non-bonded atoms are represented by the Coulomb plus Lennard-Jones terms.

\section{Stretching Energy}

Figure 43 shows an illustrative example of the stretching energy of two atoms. 


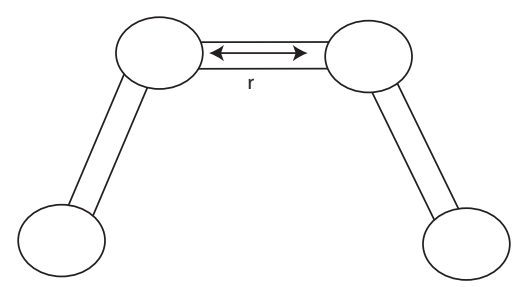

Figure 43. Schematic drawing showing the atomic configuration for bond stretching between two bonded atoms

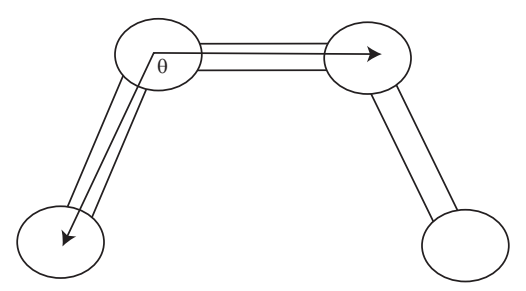

Figure 44. Schematic drawing showing the atomic configuration for bending energy of a bonded triplet of atoms

The following equation, based on Hooke's law, is used to describe the stretching energy

$$
E_{s}=\sum_{\text {bonds }} k_{b}\left(r-r_{0}\right)^{2},
$$

where $k_{b}$ controls the stiffness of the bond spring and $r_{0}$ is the equilibrium length. Unique values of $k_{b}$ and $r_{0}$ are assigned to each pair of bonded atoms based on their type (e.g. C-C, C-H, O-C, etc). Eq. (84) estimates the energy associated with the vibration around the equilibrium bond length.

\section{Bending Energy}

Figure 44 shows an illustrative example of the bending energy of a bonded triplet of atoms. Also based on Hooke's law, the following equation describes the bending energy

$$
E_{b}=\sum_{\text {angles }} k_{\theta}\left(\theta-\theta_{0}\right)^{2},
$$

where $k_{\theta}$ controls the stiffness of the angle spring and $\theta_{0}$ is the equilibrium angle. The values for $k_{\theta}$ and $\theta_{0}$ are also based on their type (e.g. C-C-C, C-O-C, C-C-H, etc). The larger the value of $k_{\theta}$, the more energy is required to deform an angle 


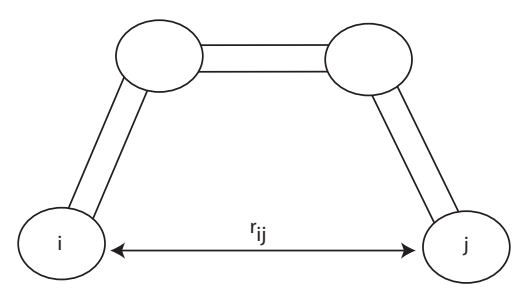

Figure 45. Schematic drawing showing the atomic configuration for non-bonded energy between non-bonded atoms $i$ and $j$

from its equilibrium value.

\section{Non-Bonded Energy}

Figure 45 shows an illustrative example of the non-bonded energy between nonbonded atoms $i$ and $j$. The non-bonded energy accounts for the repulsion, van der Waals attraction, and electrostatic interactions. The van der Waals attraction occurs at short ranges and rapidly dies off as the interacting atoms move a few Angstroms apart. This is modeled using the Lennard-Jones 6-12 potential. The electrostatic contribution is modeled using a Coulombic potential. Mathematically, this energy can be written as,

$$
E_{a b}=\sum_{i}^{\text {on }} \sum_{j}^{\text {on b }}\left\{4 \epsilon_{i j}\left[\left(\frac{\sigma_{i j}}{r_{i j}}\right)^{12}+\left(\frac{\sigma_{i j}}{r_{i j}}\right)^{6}\right]+\frac{q_{i} q_{j} k_{e}}{r_{i j}}\right\} f_{i j},
$$

where $q_{i}$ is the charge on the $i^{\text {th }}$ atom, $k_{e}$ is a proportionality constant, $\epsilon_{i j}=$ $\sqrt{\epsilon_{i i} \epsilon_{j j}}$ is the depth of the potential well, $r_{i j}$ is the distance between atoms, and $\sigma_{i j}=\sqrt{\sigma_{i i} \sigma_{j j}}$ is the distance at which the inter-particle potential is zero. Furthermore, $f_{i j}=1$ except for 1,4 -interactions in which $f_{i j}=.5$ (refer to $[91,92]$ for explanation). Eq. (85) is only counted for a series atoms three or more apart.

\section{Torsion Energy}

Figure 46 shows an illustrative example of the torsion energy between each bonded quartet of atoms. The torsion energy is modeled as a simple periodic function and is used primarily to make the total energy agree with experimental or rigorous quantum mechanical calculations for a model dihedral angle. The equation to 


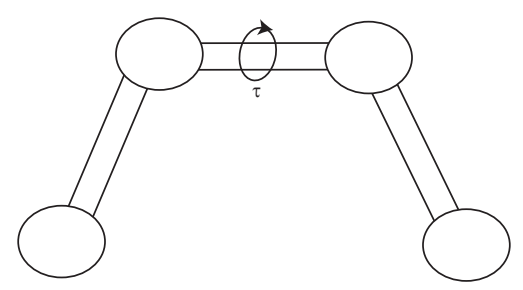

Figure 46. Schematic drawing showing the atomic configuration for torsion energy for each bonded quartet of atoms

describe the torsion energy is,

$$
E_{t}=\sum_{\text {torsions }} A[1+\cos (n \tau-\phi)]
$$

where $A$ controls the amplitude, $n$ controls the periodicity, and $\phi$ shifts the curve along the rotation axis. The parameters are assigned to each bonded quartet of atoms based on their type (e.g. C-C-C-C, C-O-C-H, H-C-C-H, etc).

\section{Total Energy}

The combination of all the above energies is the total energy, or the OPLS-AA force field. Mathematically the energy can be written as,

$$
\begin{aligned}
E=\sum_{\text {bonds }} k_{b}\left(r-r_{0}\right)^{2} & +\sum_{\text {angles }} k_{\theta}\left(\theta-\theta_{0}\right)^{2}+\sum_{\text {torsions }} A[1+\cos (n \tau-\phi)] \\
& +\sum_{i}^{\text {on a on b }} \sum_{j}\left\{4 \epsilon_{i j}\left[\left(\frac{\sigma_{i j}}{r_{i j}}\right)^{12}+\left(\frac{\sigma_{i j}}{r_{i j}}\right)^{6}\right]+\frac{q_{i} q_{j} k_{e}}{r_{i j}}\right\} f_{i j} .
\end{aligned}
$$

\subsubsection{Data Preprocessing}

As stated above, the position and velocity coordinates were recorded for each atom in the backbone chain. One drawback to using Cartesian coordinates is the rigid body motion. This will be later addressed in Section 5 on results. However, instead of using Cartesian coordinates to describe the state of the protein we can use more generalized independent coordinates.

The generalized coordinates consist of bond lengths, bond angles, and dihedral angles. Bond lengths describe the length of the bonds between atoms; bond angles 
are the angles formed by three atoms bonded together; and dihedral angles are angles between the plane formed by the first three atoms and the plane formed by the last three atoms in a four atom chain. There are three different dihedral angles describing different geometries. $\phi$ (phi, involving the backbone atoms $C^{\prime}-N-$ $\left.C^{\alpha}-C^{\prime}\right), \psi\left(\right.$ psi, involving the backbone atoms $N-C^{\alpha}-C^{\prime}-N$ ) and $\omega$ (omega, involving the backbone atoms $\left.C^{\alpha}-C^{\prime}-N-C^{\alpha}\right)$. Thus, $\phi$ controls the $C^{\prime}-C^{\prime}$ distance, $\psi$ controls the $N-N$ distance, and $\omega$ controls the $C^{\alpha}-C^{\alpha}$ distance. 


\section{CHAPTER 5}

\section{Results}

In this section, the results will be presented following the order of the previous chapter. The muscle fatigue tracking in both the subjects performing the high and low sawing motion will be presented followed by the Army walking soldiers. Lastly, the molecular dynamics will be considered.

\subsection{Fatigue Tracking-Sawing Subjects 5.1.1 High Experiment}

Out of 10 subjects, three subjects (numbers one, four, and five) were not included in the results presented here. This decision was based on two criteria: (1) the middle and anterior deltoid muscles did not show a decrease of at least

$20 \%$ from the percent of initial value in the mean frequency spectrum and (2) if there was limited dynamical structure in the kinematics when plotting with the time delay. If the middle and anterior deltoid muscles, which are two of the major muscles working in these experiments, did not show at least a limited amount of fatigue, then other muscles would have experienced even less fatigue. Thus, in these subjects, if there is limited or no fatigue to track, then our methodology will not work. If the kinematics data has no dynamical structure in the reconstructed phase space, then our methodology will not work. The loss of this dynamical structure can be attributed to (1) high contamination of noise, or (2) malfunctions in the measurement system. By using these two conditions, we may eliminate any outliers, which are not suitable for our methodology and would skew the betweensubject-variability plots.

For kinematic analysis, particulars about the data processing will be described for the humeral plain angle of subject 6 in the high sawing experiment. Time delay 

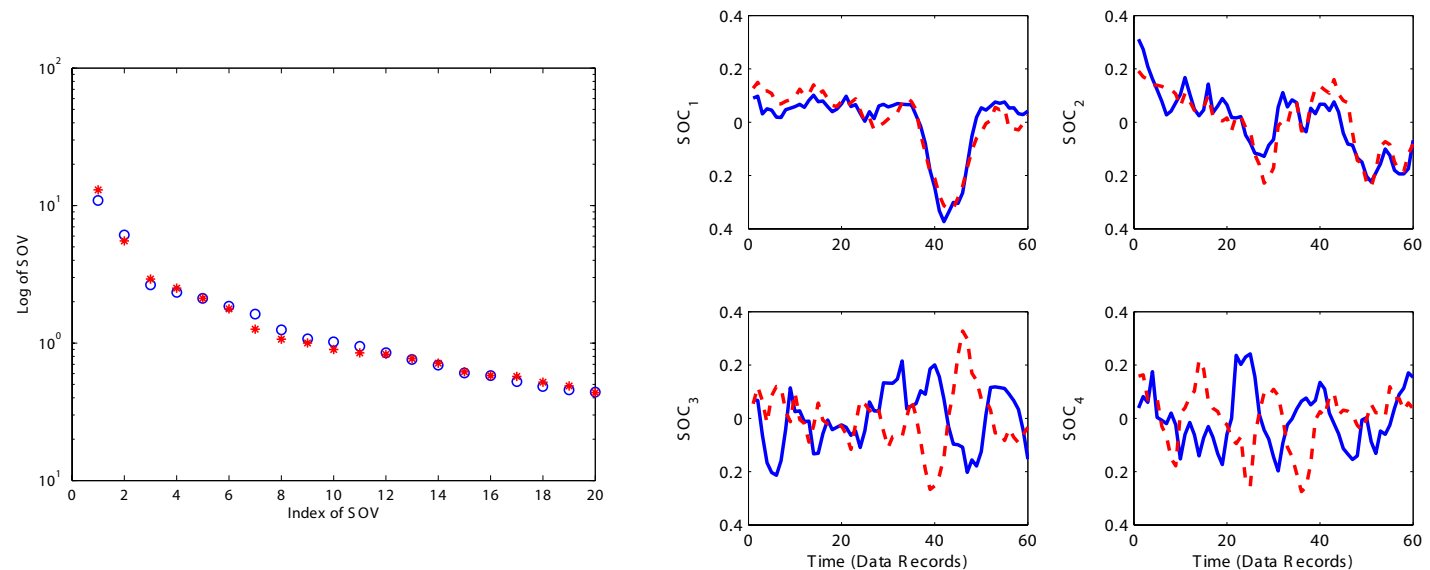

Figure 47. Left: first 20 calculated SOVs for subject 6 humeral plain angle without the STRMP weighting function (blue $\circ$ ) and with the weighting function (red *). Right: first four corresponding dominant SOCs for subject 6 humeral plain angle without the STRMP weighting function (blue, - ) and with the weighting function $(\mathrm{red},--)$

and embedding dimension were found to be 21 and 3, respectively. The kinematic time series had 53,442 points and the EMG had 961,953 points. The reference phase space was spilt into 32 hyper-boxes, and the local linear model was based on 16 nearest neighbors in the reference data (Refer to Appendix A for all subject's data). Refer to the previous chapter for the EMG figures on this subject Figs. 29, 31 and 33 .

The effects that the short-time reference model prediction (STRMP) weighting function in PSW have on SOVs and SOCs are depicted in Fig. 47. In the left plot, the SOV corresponding to the weighting $($ red $*$ ) are slightly greater in magnitude compared with no weighting function (blue o). Thus, the corresponding SOC will be smoother in time (red --). In the right plot, the first two SOCs using the weighting function are slightly smoother. However, after three and four SOCs the contamination of high frequency content becomes more visible. On average, across all subjects and angles using the STRMP weighting function produces SOVs that are slightly greater in magnitude and thus, it extracts smoother trends. For the 
remainder of this dissertation the weighting function is used in all of the results.

Figure 48 depicts the energy calculated using POD (red o) and SOD (blue *) from the PSW-based feature vectors for the elbow flexion/extension Fig. 48 (top), humeral plane angle Fig. 48 (middle), and humeral elevation angle Fig. 48 (bottom) for subject 6 . In the first column, the energy or signal power is calculated in each mode using POD and SOD. Across each angle, the energy captured in each mode by POD and SOD is very similar. For POD, the first mode captures the greatest amount of energy or signal power, which is to be expected since POD is optimal in finding energy in a signal. As the index of the mode is increased, the energy in that particular mode is decreased. However, in SOD there is not a monotonically decreasing trend of energy in each mode. In SOD, modes are ordered by smoothest in time and not by energy alone which gives rise to the jaggedness in the energy plot. For example, in the flexion/extension angle the second mode captures more energy than the first mode, however, the first mode should be representative of being the smoothest in time and not only containing the maximum energy (i.e., variance). Then, the sixth mode captures more energy than the past several modes.

The second column is the percent of the total energy each mode contains. For POD, the first mode captures a large percentage of the energy and the first several modes captures a majority of the energy. However, with SOD, the percentage of total energy that is captured in each mode when compared to POD is very small. Clearly, this can also be seen from the magnitude of the values in the first column.

The last column of Fig. 48 shows the energy in each POD and SOD subspace. Since the proper orthogonal modes are orthogonal, the subspace plot is found by the cumulative sum of the proper orthogonal values (POVs). However, the smooth projection modes are not orthogonal, only linearly independent. Therefore, the modes are transformed to a set of orthornormal basis vectors. The sum of the 
POVs of the particular subspaces spanned by the orthornormal basis vectors yields the energy in each SOD subspace. The energy in each POD and SOD subspace is very similar with both trends very close to each other. POD captures more energy in each subspace until roughly 20 dimensions where the trends plateau. At this point, both methods capture the same energy. Figure 49 depicts the extracted
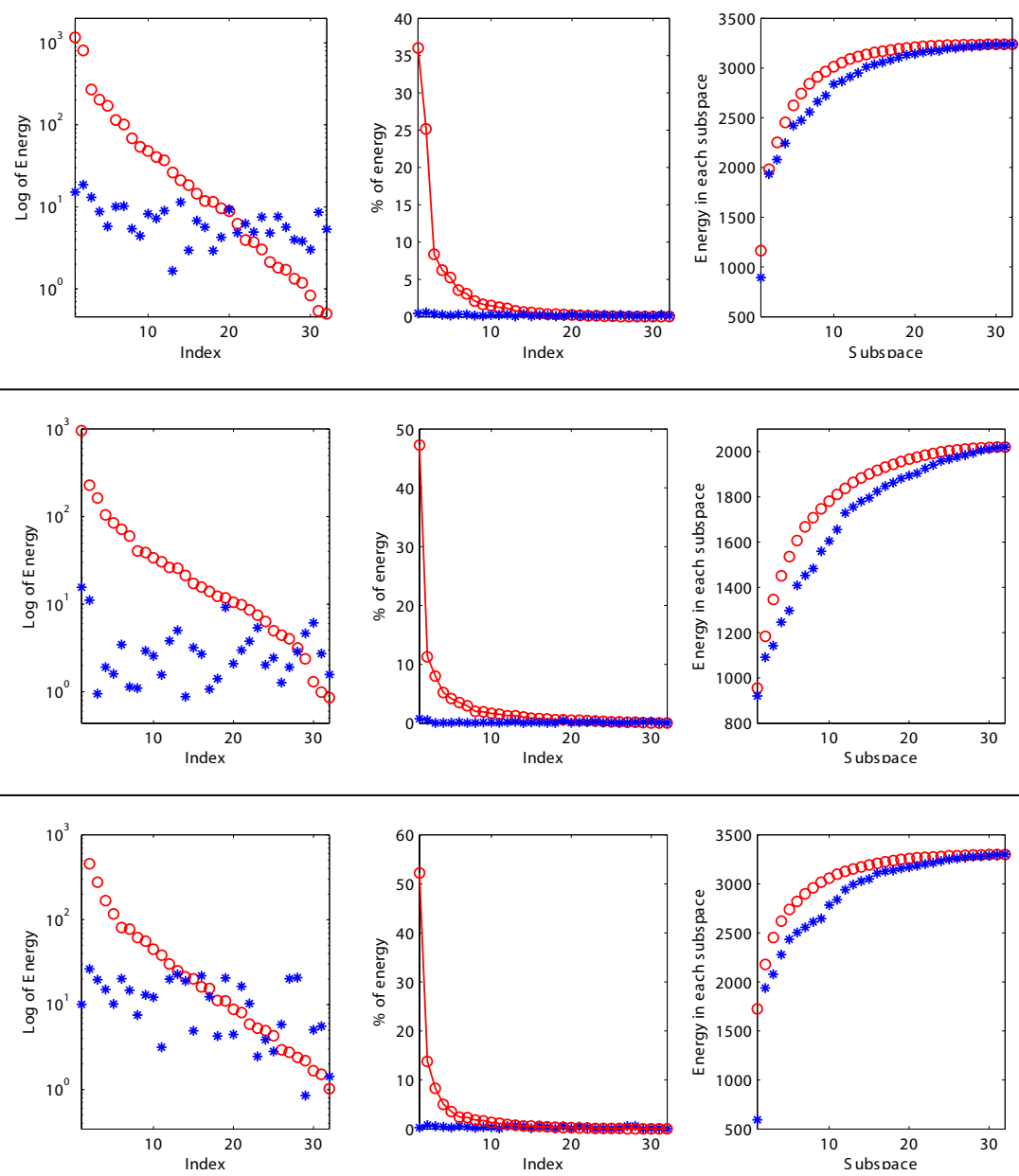

Figure 48. Plots showing the energy captured by POD (red o) and SOD (blue *) for subject 6 elbow flexion/extension (top), humeral plane angle (middle), and humeral elevation angle (bottom) for the high sawing experiment. The first column is the energy captured in each mode, the second column is the percent of total energy captured by each mode, and the last column is the energy captured in each subspace.

first four coordinates using SOD (red) and POD (blue). From the figure, the smoothness in the SOCs is very obvious. The POCs are reasonably close to the 
same trend as the SOCs expect for the jaggedness or lack of smoothness. The third and fourth SOC and POC start to approach each other with similar behavior and jaggedness. However, the SOCs are still a little smoother than the POCs. Since it is assumed that muscle fatigue is a smooth deterministic process than the SOCs should be able to capture the fatigue in a lower dimensional subspace than if only energy is used. Therefore, for this particular application, smoothness may be a better characteristic in tracking the fatigue than energy.

Figure 50 depicts results from both linear and nonlinear (quadratic and cubic expansions) SOD analysis of the elbow flexion/extension, humeral plane angle, and humeral elevation angle PSW features. By looking at linear SOVs across all angles there are roughly at most three modes that are significantly different from the rest of the modes. This is seen in the elbow flexion/extension angle with two modes that are slightly separated from the rest. In the case where there are dominant modes, which can be defined as at least an order of magnitude separation, there are about one or two modes. However, some additional slow-time information could leak into higher-order linear modes. Therefore, five linear SOCs were used in NSOD analysis for both quadratic and cubic expansions.

Only the first 20 SOVs are shown in Fig. 50 with the corresponding four most dominant smooth coordinates (SOCs). From SOV plots, it is clear that the nonlinear analysis provides SOVs that are greater in magnitude than their linear counterparts, cubic SOVs being the largest. This can also be seen in the corresponding smooth coordinates, where the nonlinear coordinates in all three figures are smoother than the corresponding linear coordinates, cubic coordinates showing considerably smoother trends. Referring to Fig. 50, there is a clear separation between the first one or two dominant SOVs and the point where the values start falling off to the noise floor (indicating high frequency contamination). This is 
clearly seen in the corresponding smooth coordinates also: as the SOC index increases, more high frequency content is present.

Coefficient of determination, $R^{2}$, values are used to determine how well SOCbased fits match the actual EMG-based fatigue markers. For each set of SOCs, monotonically increasing number of SOCs are projected onto MNF trends in the least squares sense. An ad hoc F-test $(p<0.05)$ is used to tell how many SOCs are required to adequately describe the local muscle fatigue EMG trends. If the projections track the EMG well, meaning an $R^{2}$ value is close to one, than there is a one-to-one map from the corresponding SOC space to the particular EMG space.

Figure 51 depicts the between-subject-variability in $R^{2}$ values for the SOC fits onto local MNF trends. Across all subjects and muscles, it is evident that the NSOD based fits have consistently higher $R^{2}$ values compared to the linear SOD results, with cubic SOD fits showing the largest $R^{2}$ values. In addition, cubic coordinates also show a significant decrease in the variability or a smaller standard deviation in the $R^{2}$ value across all subjects.

The most significant improvement in the quality of fit is seen in the deltoid muscles where there are 20 coordinates needed for linear SOD. This is analogous to needing a 20-dimensional coordinate subspace - or 20-dimensional SOC subspaceto capture the EMG. For cubic SOD, only 3 or 4 coordinates are needed. This drastic improvement is also seen in the flexor carpi radialis muscle. This is an order of magnitude reduction in the number of coordinates or dimensions needed to track the EMG. In all muscles, cubic SOD analysis outperforms linear SOD analysis in the number of dimensions needed to track EMG.

The corresponding projections of both linear and nonlinear SOCs onto the local EMG trends are shown to illustrate how well SOC fits match the actual EMG trends for subject 6, Fig. 52 (Refer to Appendix B for all subjects). The 
number of SOCs in each projection are labeled on the top of Fig. 52. The first thing to notice is how much smoother the nonlinear tracking is compared to linear tracking in almost all cases, with cubic SOC fits being smoothest. Again, this relates to the improved degree of smoothness exhibited by the nonlinear SOCs. Also, we can see that the nonlinear fits more closely track the actual EMG trend while needing fewer coordinates. 

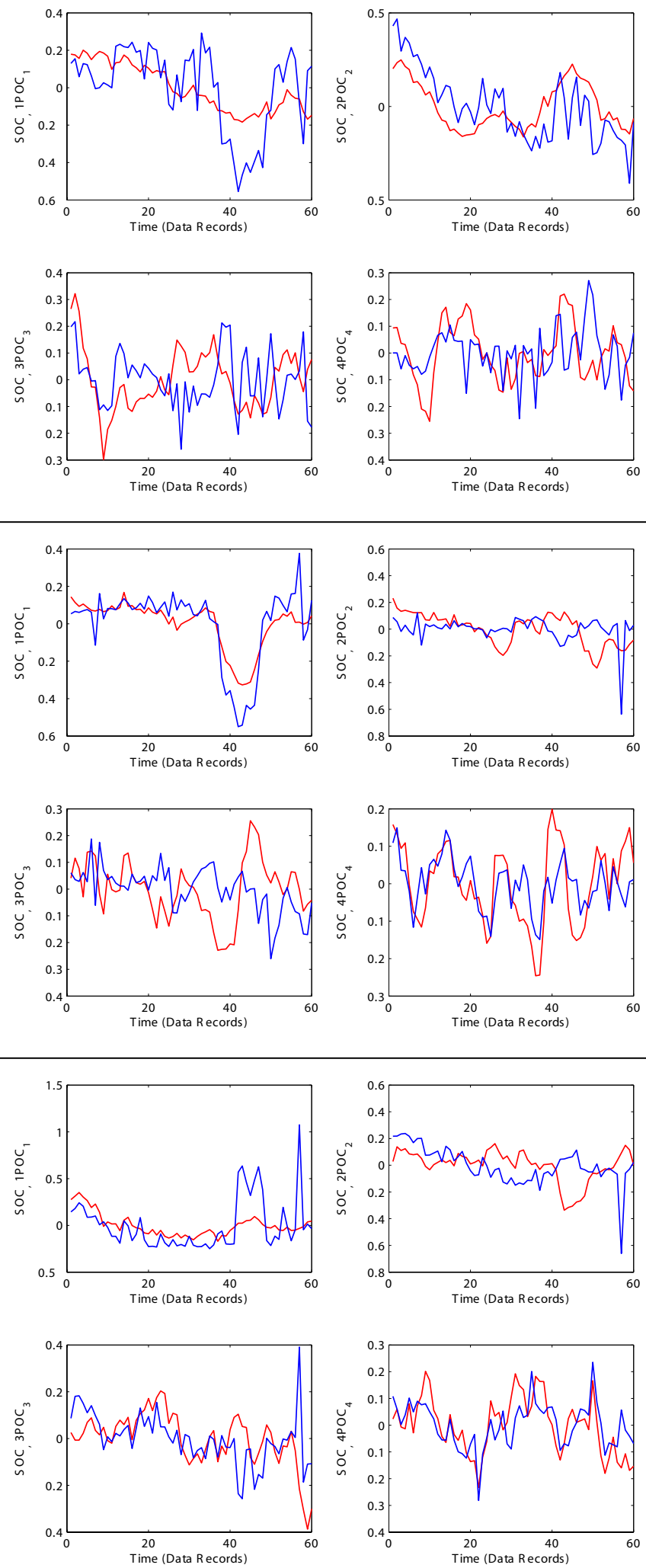

Figure 49. Plots comparing calculated SOCs (red) and POCs (blue) for subject 6 elbow flexion/extension (top), humeral plane angle (middle), and humeral elevation angle (bottom) for the high sawing experiment. 

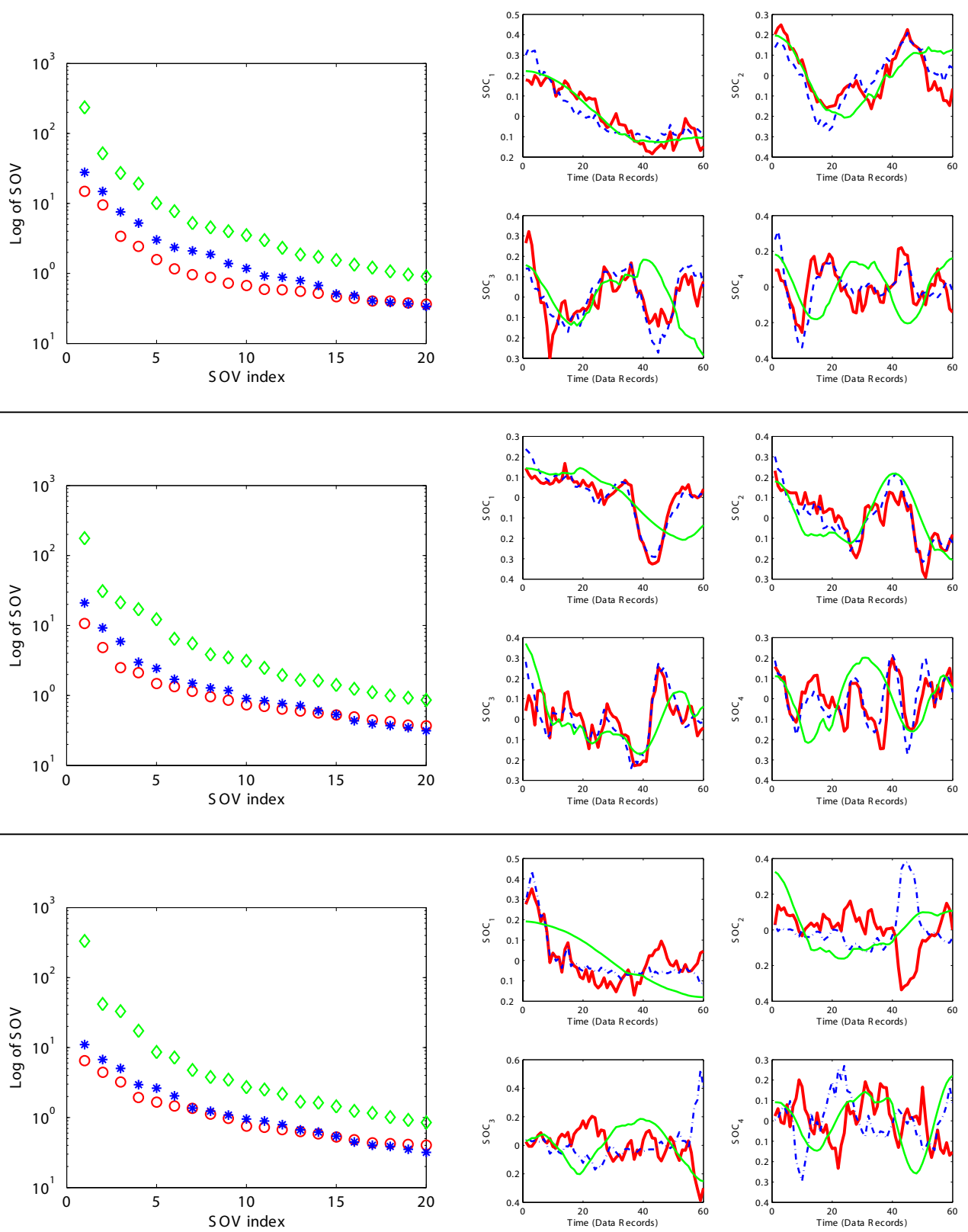

Figure 50. Left plots: first 20 dominant linear (red o), quadratic (blue $*$ ), and cubic (green $\diamond)$ SOVs for subject 6 elbow flexion/extension (top), humeral plane angle (middle), and humeral elevation angle (bottom) for the high sawing experiment. The corresponding first four dominant linear (red, thick -), quadratic (blue, thin -- ), and cubic (green, thin -) (plots on the right). 

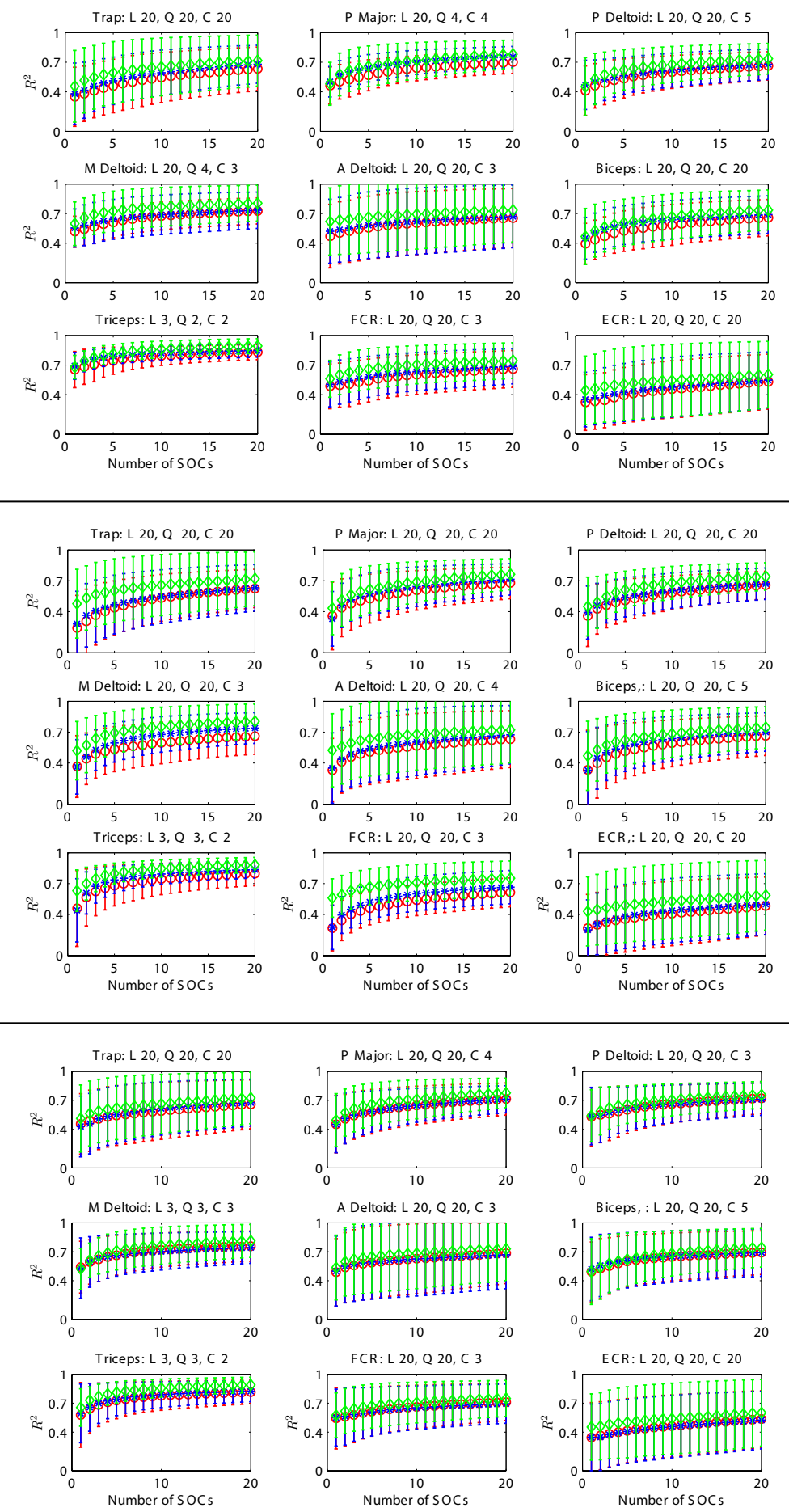

Figure 51. Between subject variability $R^{2}$ values for MNF trends in the high sawing experiment for linear (red $\circ$ ), quadratic (blue $*$ ), and cubic (green $\diamond$ ) SOCs. Error bars represent one standard deviation from the mean of $R^{2}$. Elbow flexion/extension (top), humeral plane (middle), and humeral elevation (bottom) angles. Values on top of each plot indicate the number of SOCs needed to adequately track EMG trends as determined from the F-test. 

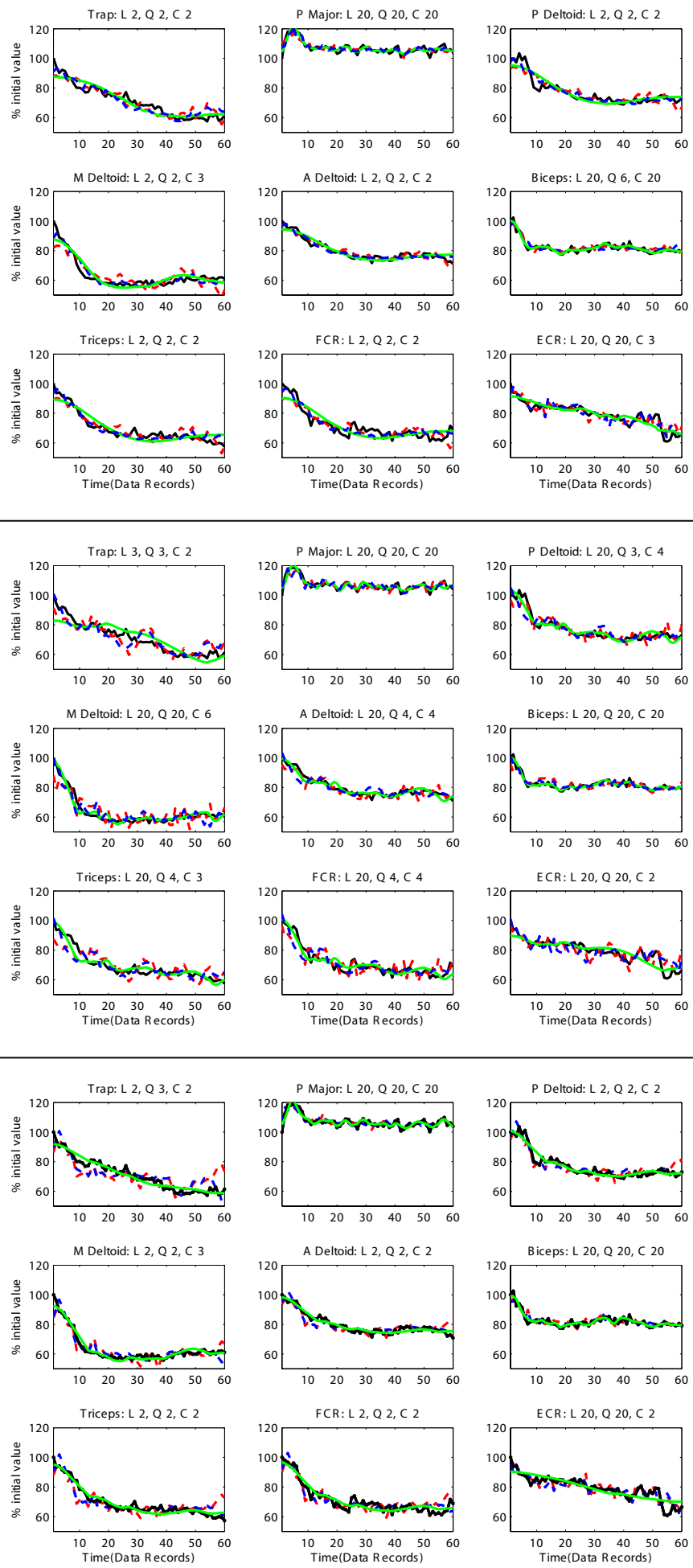

Figure 52. Linear combinations of linear (red --), quadratic (blue --), and cubic (green - ) SOCs projections onto MNF (black - ) markers in a least squares sense for subject 6 high sawing experiment: elbow flexion/extension (top), humeral plane (middle), and humeral elevation (bottom) angles. 


\subsubsection{Low Experiment}

For the low sawing experiment, the analysis of the data is the same so particulars will not be described. As in the high sawing experiment, 5 slow-time modes are reserved for the nonlinear SOD analysis.

The energy is depicted for subject 6 in the elbow flexion/extension Fig. 53 (top), humeral plane angle Fig. 53 (middle), and humeral elevation angle Fig. 53 (bottom). The results and conclusions are the same from the high sawing experiment. POD is able to capture a majority of the energy in the first several modes. However, SOD again does not capture such a large amount of energy in the first several modes since the method also considers the smoothness of the signal. The energy in each subspace is again very similar. In the flexion/extension, a onedimensional subspace captures almost the same amount of energy. However, as the subspace dimension is increased, POD again captures slightly more energy. Both trends start to plateau at roughly a 20-dimensional subspace.

Figure 54 depicts the extracted first four coordinates using SOD (red) and POD (blue). As in the high sawing experiment, the same results are seen. SOD extracts much smoother trends than POD which considers only energy. As the index of the coordinates are increased, the SOCs become contaminated with more high frequency content and the POCs approach the SOCs smoothness. Still the lower dimensional SOCs exhibit a much smoother behavior and should track the fatigue in a lower dimensional subspace.

Figure 55 depicts results from both linear and nonlinear (quadratic and cubic expansions) SOD analysis of the elbow flexion/extension, humeral plane angle, and humeral elevation angle PSW features. Only the first 20 SOVs are shown in Fig. 55 with the corresponding four most dominant smooth coordinates (SOCs). From the plots, linear and quadratic SOD have similar SOV values. However, there 
is some clear separation in the first and second SOVs. The most drastic separation is seen in the humeral elevation angle. This is seen in the corresponding SOCs that have roughly the same smoothness. However, SOVs from cubic SOD are distinctly greater than linear and quadratic SOVs. Furthermore, this is apparent in the cubic SOCs which exhibit a much greater smoothness.

Figure 56 depicts the between subject variability in $R^{2}$ values of the SOC fits onto local MNF trends. Across all subjects and muscles, it is evident that the NSOD based fits have consistently higher $R^{2}$ values when compared to the linear SOD results. Linear and quadratic SOD results are very similar across all muscles and angles. For most of the angles, 20 linear and even quadratic SOCs are needed to capture the EMG trend. However, cubic SOD shows significant improvement in various angles, in addition, showing a decrease in the variability of the quality of fits.

Across all angles, the triceps muscle shows an order of magnitude reduction in the number of coordinates needed. The humeral elevation angle shows approximately 20 dimensions from linear to cubic SOD. The elbow flexion/extension angle shows the most improvement in the muscles from linear to cubic SOD. This is seen in the trapezius, pectoralis major, posterior deltoid, and triceps muscles. Cubic SOD for the elbow flexion/extension requires the lowest dimension to track the Triceps muscle.

The corresponding projections of both linear and nonlinear SOCs onto the local EMG trends are shown to illustrate how well SOC fits match the actual EMG trends for subject 6 (Fig. 57) (Refer to Appendix B for all subjects). The first thing to notice is how much smoother the nonlinear tracking is compared to the linear in almost all cases, with cubic SOC fits being smoothest. Again, this relates to the improved degree of smoothness exhibited by the nonlinear SOCs. 
Also, we can see that the nonlinear fits more closely track the actual EMG trend while needing fewer coordinates. 

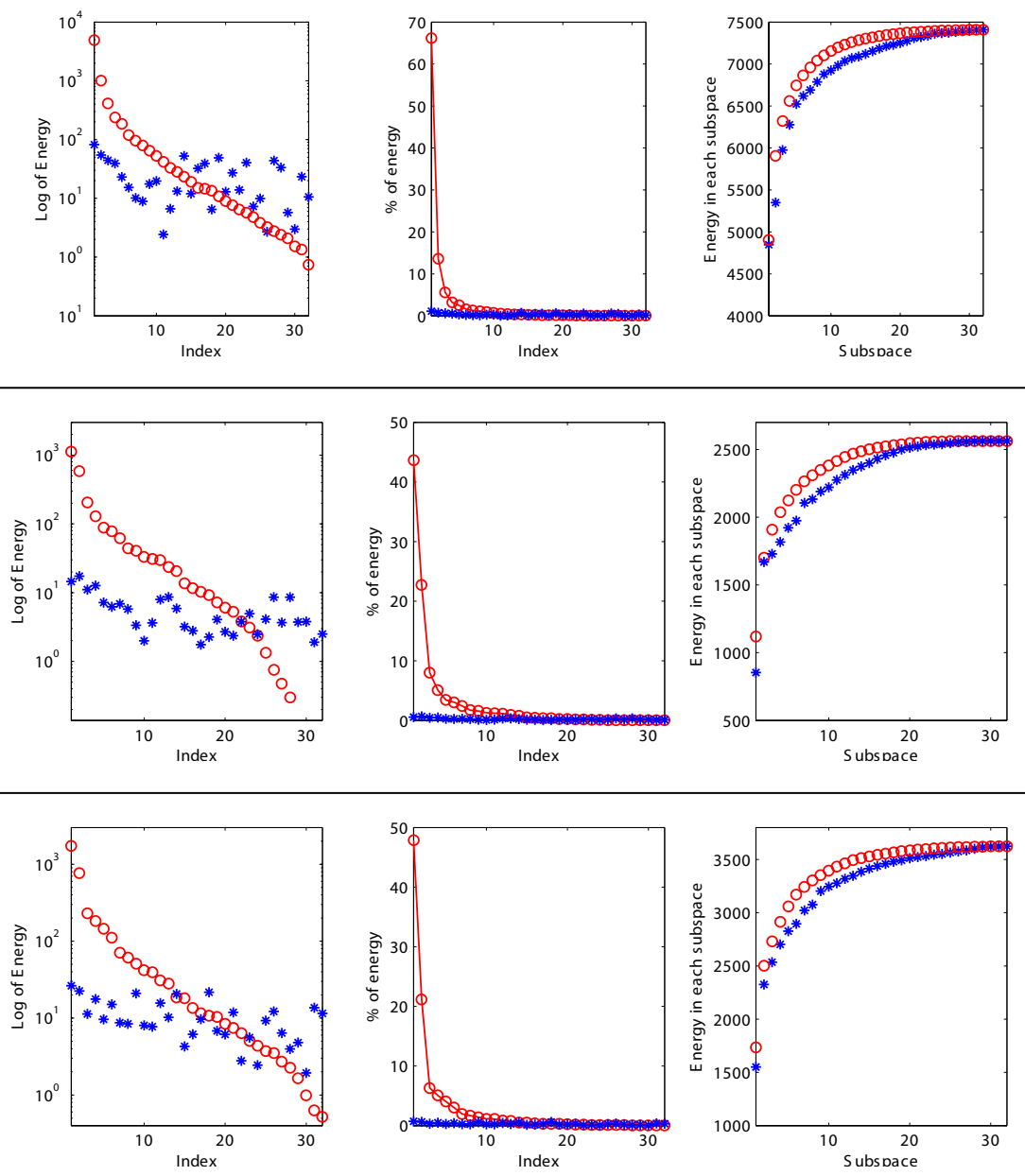

Figure 53. Plots showing the energy captured by POD (red o) and SOD (blue *) for subject 6 elbow flexion/extension (top), humeral plane angle (middle), and humeral elevation angle (bottom) for the low sawing experiment. The first column is the energy captured in each mode, the second column is the percent of total energy captured by each mode, and the last column is the energy captured in each subspace. 

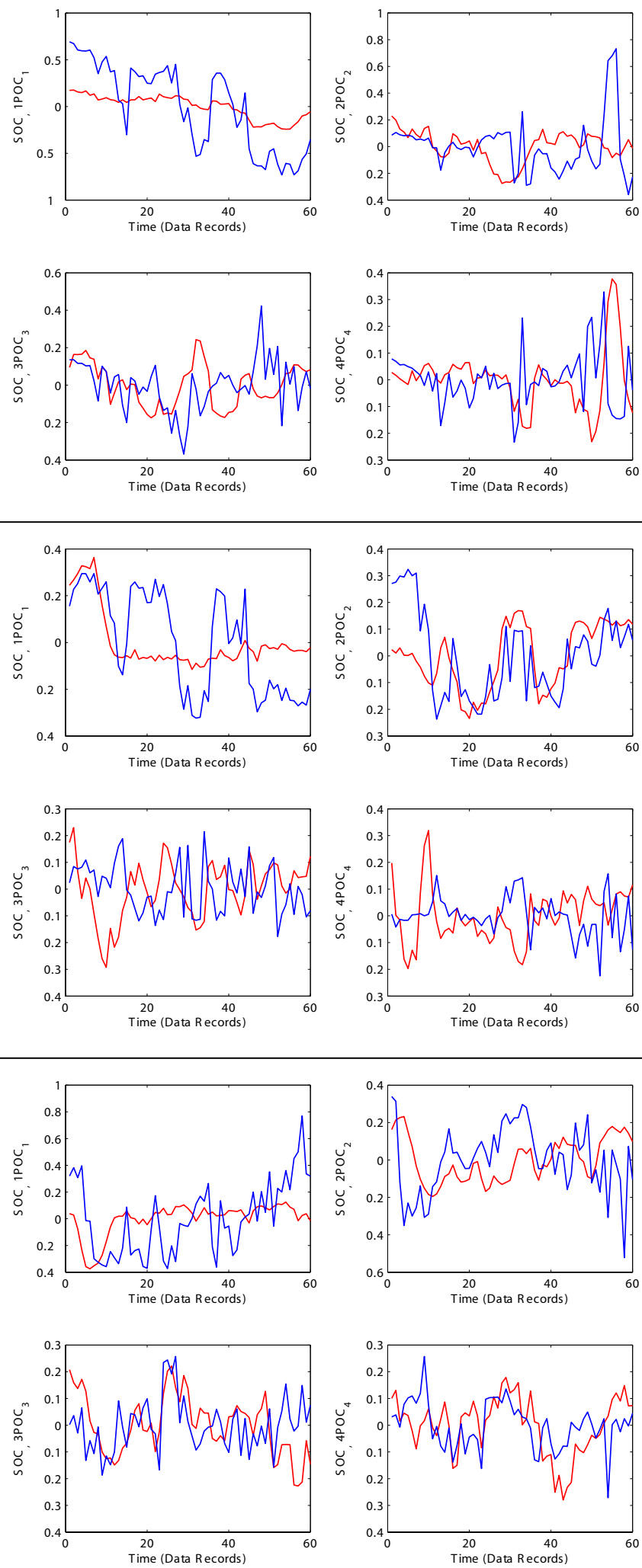

Figure 54. Plots comparing calculated SOCs (red) and POCs (blue) for subject 6 elbow flexion/extension (top), humeral plane angle (middle), and humeral elevation angle (bottom) for the low sawing experiment. 

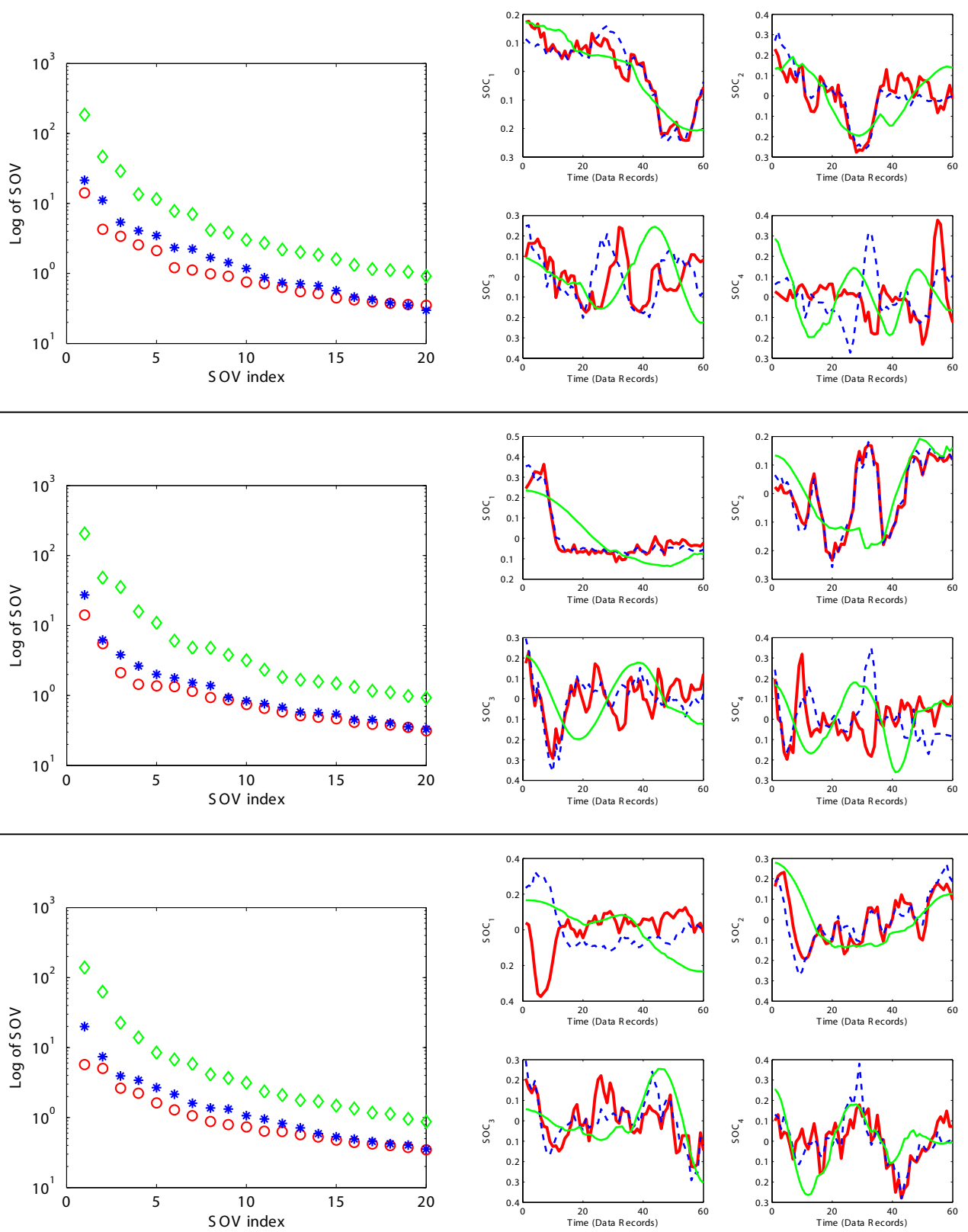

Figure 55. Left plots: first 20 dominant linear (red o), quadratic (blue *), and cubic (green $\diamond)$ SOVs for subject 6 elbow flexion/extension (top), humeral plane angle (middle), and humeral elevation angle (bottom) for the low sawing experiment. The corresponding first four dominant linear (red, thick -), quadratic (blue, thin -- ), and cubic (green, thin -) (plots on the right). 


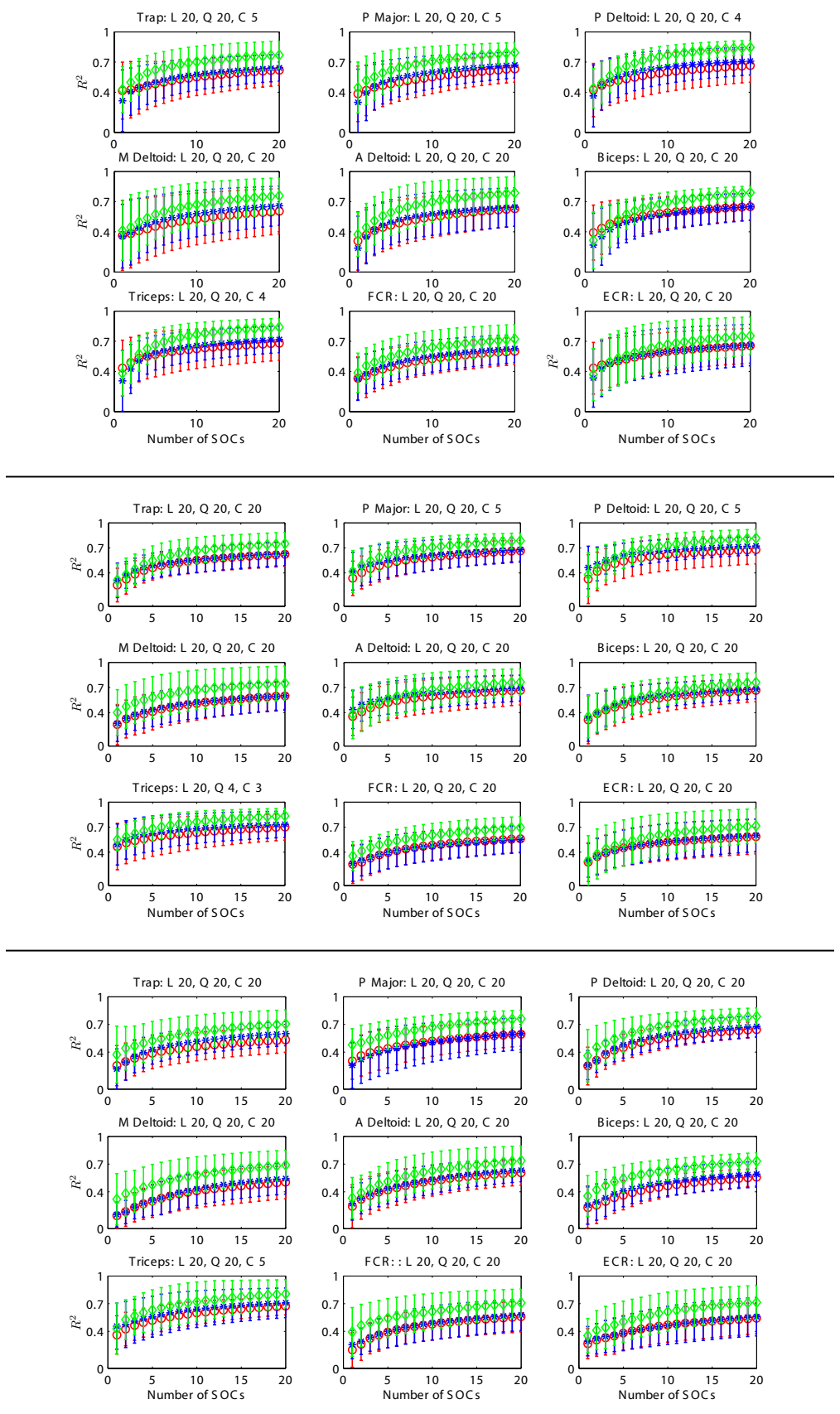

Figure 56. Between subject variability $R^{2}$ values for MNF trends in the low sawing experiment for linear (red $\circ$ ), quadratic (blue $*$ ), and cubic (green $\diamond)$ SOCs. Error bars represent one standard deviation from the mean of $R^{2}$. Elbow flexion/extension (top), humeral plane (middle), and humeral elevation (bottom) angles. Values on top of each plot indicate the number of SOCs needed to adequately track EMG trends as determined from the F-test. 

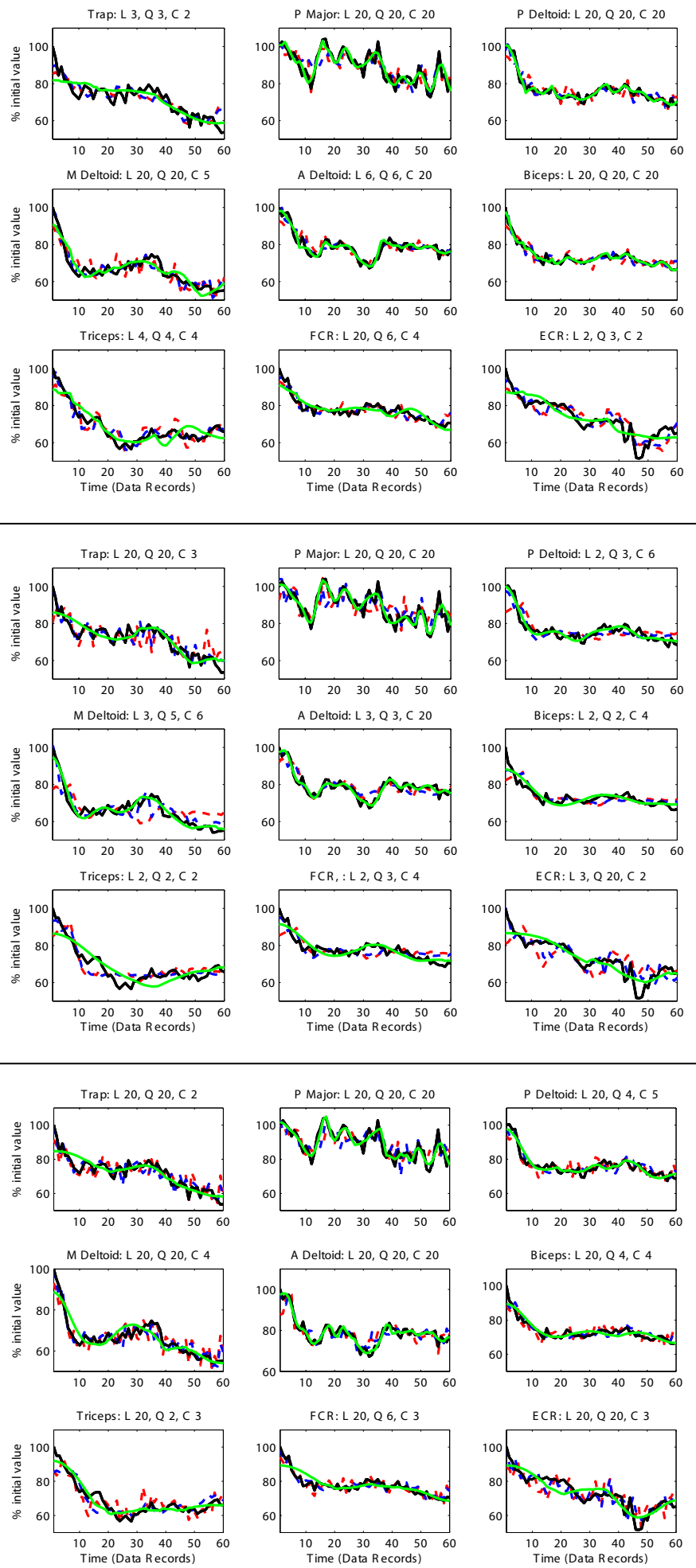

Figure 57. Linear combinations of linear (red --), quadratic (blue --), and cubic (green - ) SOCs projections onto MNF (black - ) markers in a least squares sense for subject 6 low sawing experiment: elbow flexion/extension (top), humeral plane (middle), and humeral elevation (bottom) angles. 


\subsection{Fatigue Tracking-Army's Walking Subjects}

The results from the sawing subjects show that nonlinear SOD is able to capture fatigue related trends in a lower-dimensional space. Therefore, after showing the SOD analysis of the kinematic angles, only the cubic nonlinear SOCs will be used. Also, since there are only three subjects, more individual subject results will be presented since cross subject variability may not produce an accurate account of the results.

The energy captured by POD and SOD is depicted in Fig. 58 for subject 10 lean Fig. 58 (top), hip Fig. 58 (middle top), ankle Fig. 58 (middle bottom), and knee Fig. 58 (bottom) angles. The results across all three plots parallel that of both the sawing experiments. One thing to notice is in the lean angle (TOP) the variation in SOD is much less. Also, the index where the energy in the SOD modes is greater then that of the POD mode is much less. The same is seen for the hip angle (middle top). Also, in the last column, the number of dimensions in the subspace of where the trend starts to plateau is significantly smaller at roughly a 7-dimensional subspace. This is approximately an order of magnitude less then all other angles and experiments.

Figure 59 depicts the extracted first four coordinates using SOD (red) and POD (blue). The results are very similar to that of the sawing experiments. However, the first POC tracks very close to the SOCs for the lean (top) and hip (middle top) angles. For these two angles, the point where more energy is captured in the SOD mode then the POD mode was lower then the other angles and experiments. Therefore, for these particular angles, the energy could contribute more. In the higher-order coordinates, the results parallel that of the sawing experiment and the other angles.

The results of the kinematic analysis are depicted in Fig. 60, where the 20 
largest SOVs are shown along with the first four dominant SOCs. In each case, there is slight improvement between linear and quadratic SOVs with the greatest separation in the ankle angle. This is seen in the corresponding SOCs which are very similar to each other in the degree of smoothness. Cubic SOD shows the greatest SOV value and, as a consequence, the smoothest SOCs. This is seen across all angles. The hip and ankle angles show the greatest separation between the first two cubic modes. In the other two angles there is not a clear separation or dominant mode.

Between-subject-variability $R^{2}$ values of nonlinear coordinates projected onto IMNF trends are depicted in Fig. 61. The nonlinear SOCs track the right vastus medialis muscle requiring the least amount of coordinates across all angles. The lean angle needs four dimensions, whereas, the hip, knee, and ankle angles need only three dimensions. The lean angle is also able to track the left tibialis femoris with three dimensions. The $R^{2}$ values for the left tibialis femoris has a very small standard deviation suggesting that the SOCs track the EMG as well in each angle.

Focusing more on subject 10, Fig. 62 shows the $R^{2}$ values for nonlinear coordinates projected onto IMNF trends. Clearly, the number of dimensions needed in almost all instances is largely decreased. Across all angles the right tibialis femoris, bilateral vastus medialis, and left gastrocnemius need roughly two coordinates, whereas, the other angles still need 20. The asymmetrical movement of the body is also apparent in the plots. The left and right side of the body changes dramatically with respect to the needed coordinates to track the EMG which agrees physiologically with gait movements in humans, [95]. Furthermore, we are recording movement kinematics from only the right side of the body and taking EMG from both the left and right side of the body. The need for 20 coordinates could also be attributed to trying to reconstruct the EMG trends from the left side of 
the body using the kinematics from the right side of the body.

Figure 63 (refer to Appendix $\mathrm{C}$ for all subjects) depicts the nonlinear coordinates projected onto the IMNF trends in the least squares sense. The number of coordinates used where taken from the values provided from Fig. 62. In the muscles where only two and three coordinates are needed the SOCs track the EMG trend very well. These muscles show a significant amount of fatigue in the IMNF trend and therefore the nonlinear coordinates are able to track the trend with greater accuracy. In the other muscles, the SOCs are able to track the muscle with as much accuracy, however, 20 coordinates are needed.

Figure 64 shows the results of $\dot{V} O_{2}$ data analysis for all subjects. The vertical range in these plots are kept the same to show relative variances in each of the trends. $\left\langle\dot{V} O_{2}\right\rangle$ was estimated for the time windows corresponding to the kinematic data records. Subject 7 mean $\dot{V} O_{2}\left(\left\langle\dot{V} O_{2}\right\rangle\right)$ curve shows no discernable deterministic trend over time. Subject 8 and 10 show clearly increasing trends that saturate at the end. However, the mean trend from subject 10 displays more dramatic overall variance. From subjective evaluation it was determined that subject 10 was more physically fit and had a higher $\dot{V} O_{2 \max }$ when compared to subjects 7 and 8 . Since the task parameters were set constant over the subjects, it appears that subjects 7 and 8 started the task very close to their $\dot{V} O_{2 \max }$, while subject 10 was well below that level.

The number of nonlinear SOCs required for adequate tracking of $\left\langle\dot{V} \mathrm{O}_{2}\right\rangle$ trends across all subjects were estimated by evaluating the quality of fits using $R^{2}$ values, Fig. 65. Across all three subjects, the hip and lean angles need 20 coordinates, however, the ankle and knee angles only need three coordinates. Also, the standard deviation is much smaller in the ankle and knee angles.

The nonlinear SOCs are projected onto the estimated $\left\langle\dot{V} \mathrm{O}_{2}\right\rangle$ trends for each 
subject in Fig. 66. The values on top of each plot indicate the required number of SOCs to adequately track the $\left\langle\dot{V} O_{2}\right\rangle$ trends. The plots clearly show that for subject 10, only two coordinates are needed whereas 20 coordinates are needed for subjects 7 and 8 . The difference in these results for each subject can be attributed to $\dot{V} O_{2}$ not adequately capturing the fatigue in subject 7 and 8 . 

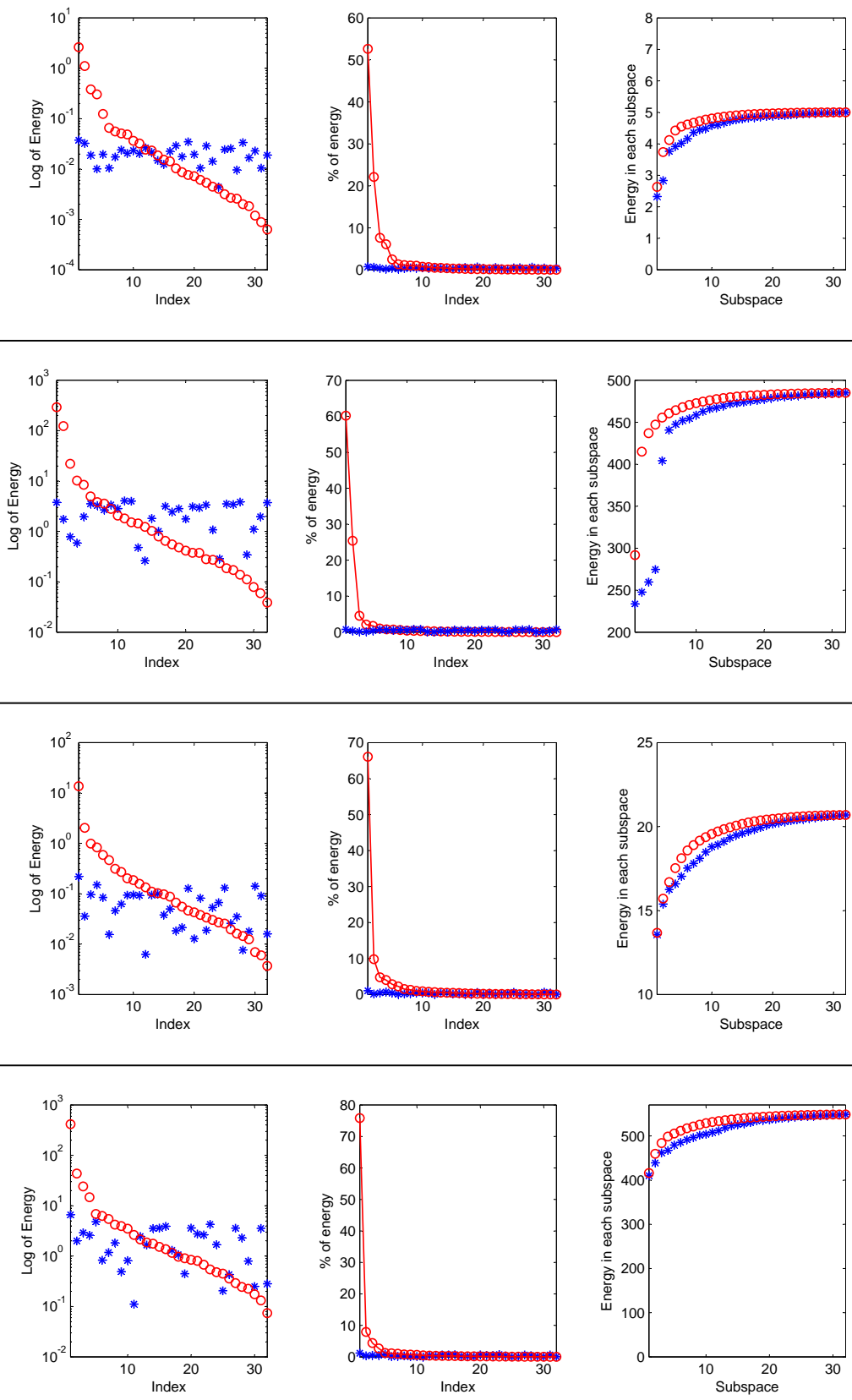

Figure 58. Plots showing the energy captured by POD (red o) and SOD (blue *) for subject 10 lean (top), hip (middle top), ankle (middle bottom), and knee (bottom) angles in the army walking experiment. The first column is the energy captured in each mode, the second column is the percent of the total energy captured by each mode, and the last column is the energy captured in each subspace. 

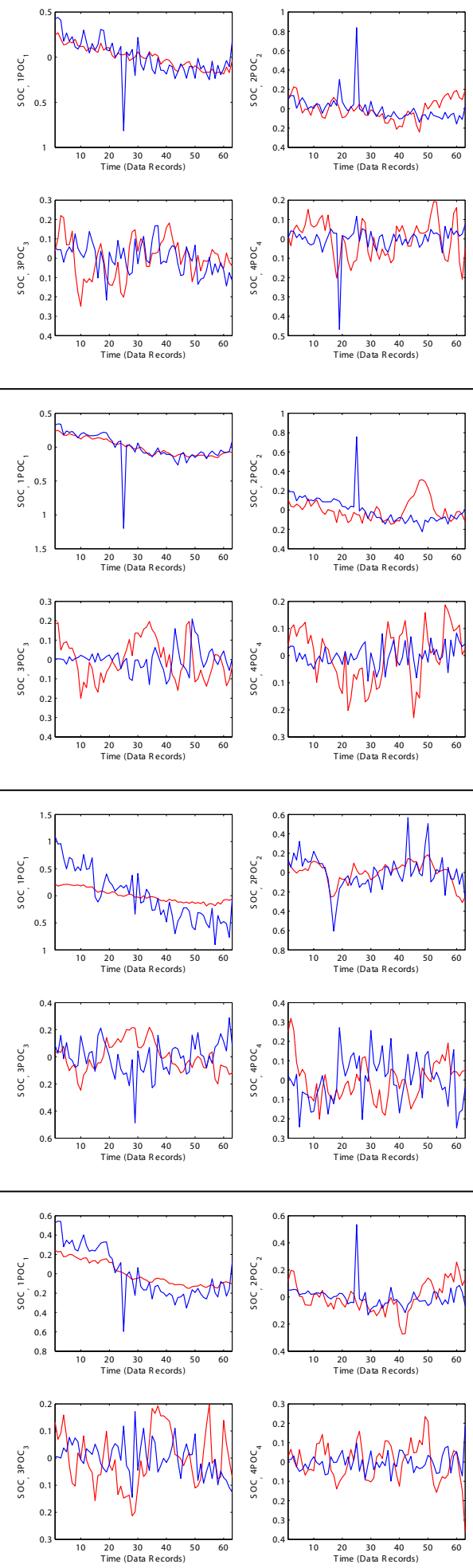

Figure 59. Plots comparing calculated SOCs (red) and POCs (blue) for subject 10 lean (top), hip (middle top), ankle (middle bottom), and knee (bottom) angles in the army walking experiment. 

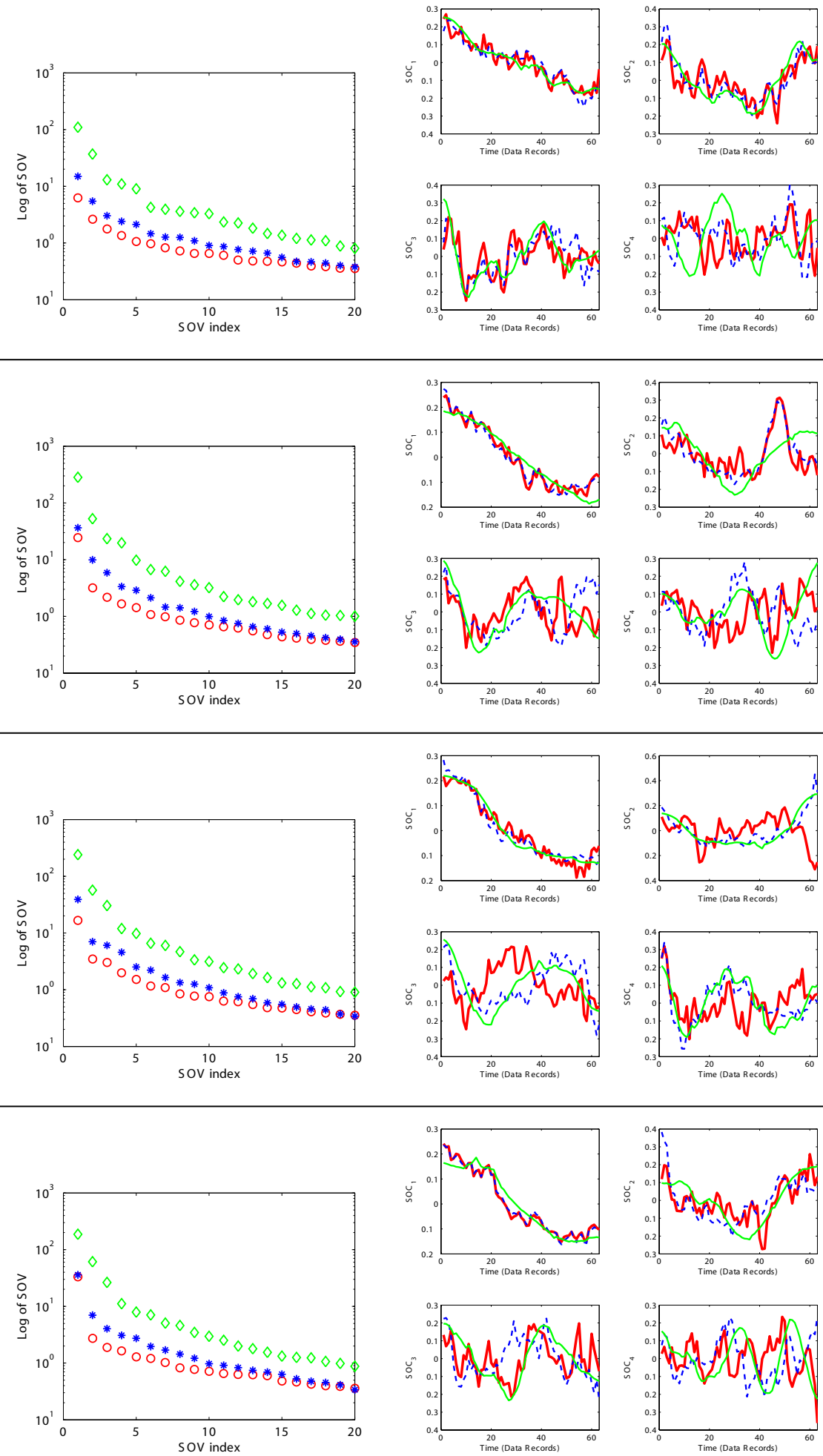

Figure 60. Left plots: first 20 dominant linear (red $\circ$ ), quadratic (blue $*$ ), and cubic (green $\diamond$ ) SOVs for subject 10 lean (top), hip (middle top), ankle (middle bottom), and knee (bottom) angles in the army walking experiment. The corresponding first four dominant linear (red, thick -), quadratic (blue, thin --), and cubic (green, thin -) (plots on the right). 

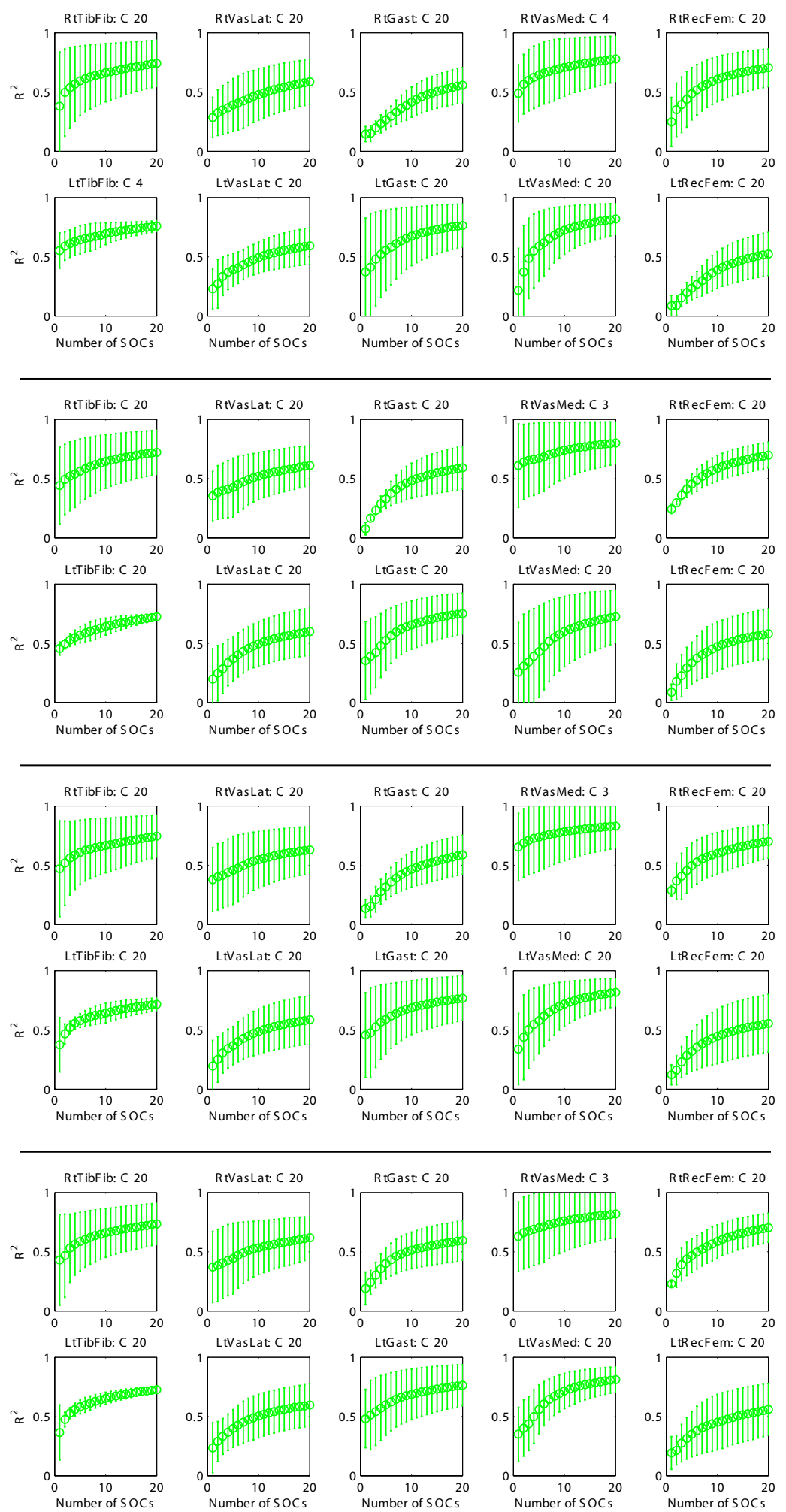

Figure 61. Between subject variability in $R^{2}$ values for IMNF trends for cubic SOCs. Error bars represent one standard deviation from the mean of $R^{2}$ for lean (top), hip (middle top), ankle (middle bottom), and knee (bottom) angles in the army walking experiment. Values on top of each plot indicate the number of SOCs needed to adequately track EMG trends as determined from the F-test. 

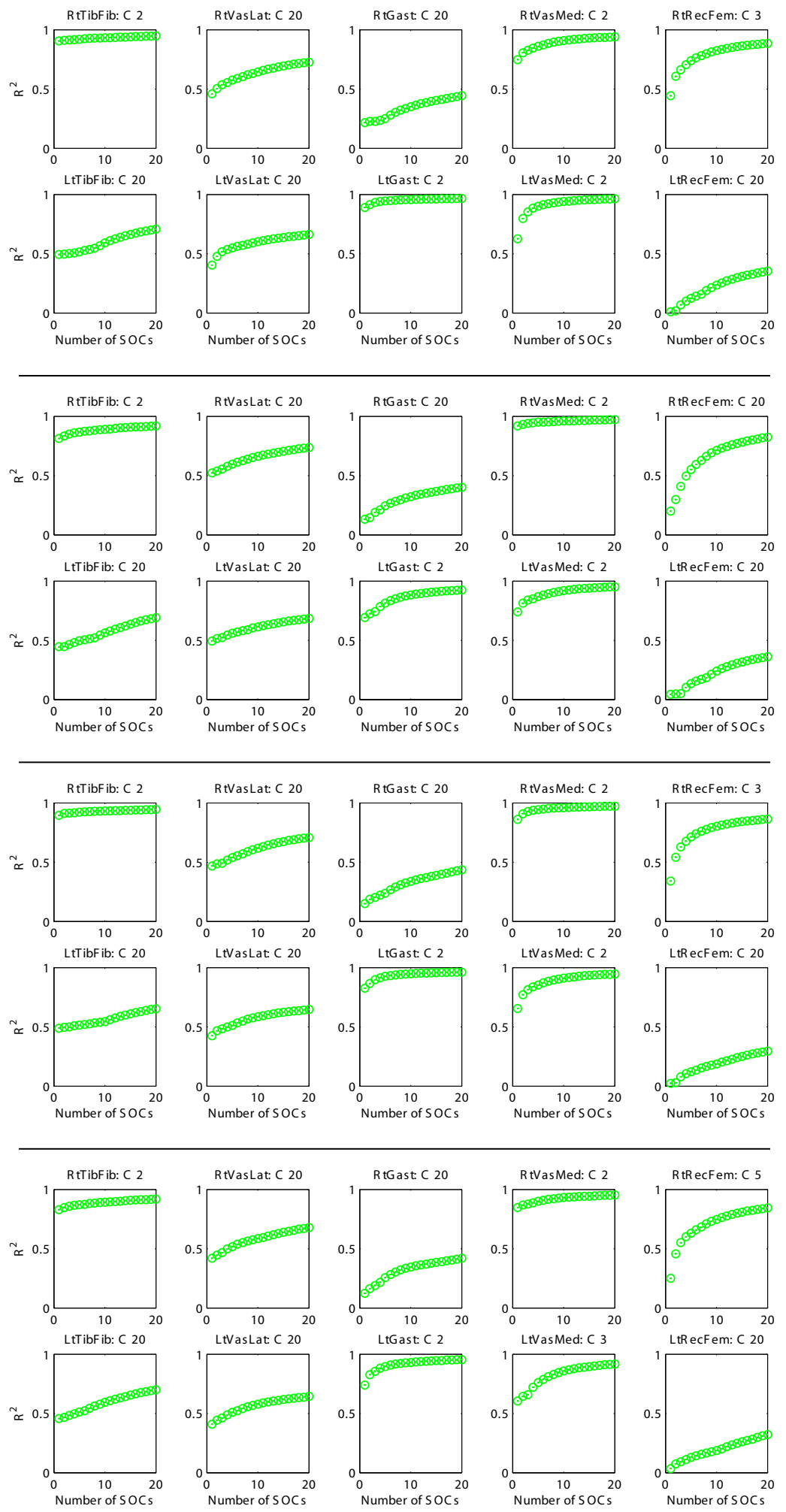

Figure 62. $R^{2}$ values for IMNF trends for cubic SOCs for subject 10 in the army walking experiment for lean angle (top), hip angle (middle top), ankle angle (middle bottom), and knee angle (bottom). Values on top of each plot indicate the number of SOCs needed to adequately track EMG trends as determined from the F-test. 

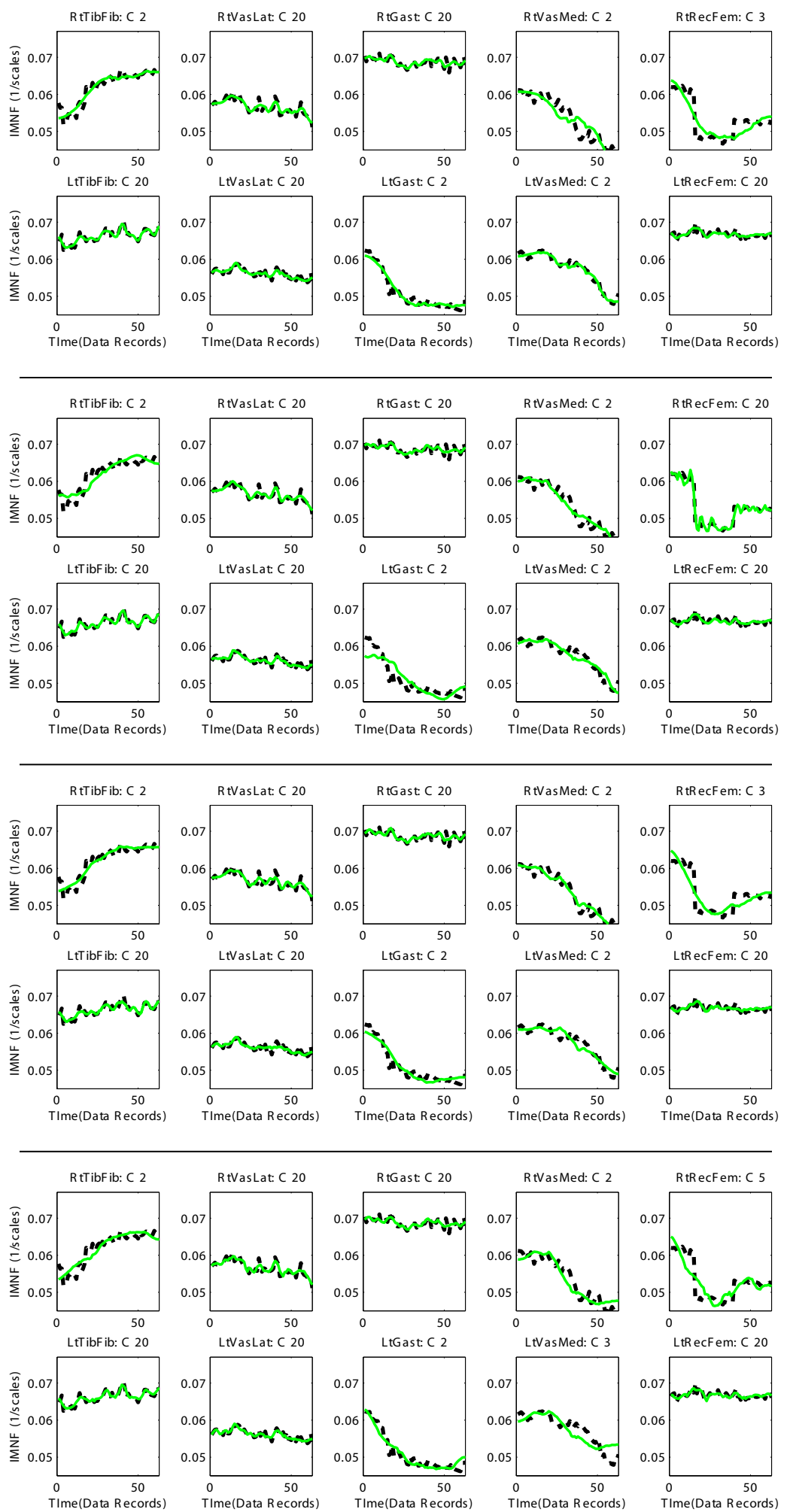

Figure 63. Linear combinations of nonlinear SOCs (green, thin -) projected onto IMNF trends (black, thick--) in a least squares sense for subject 10: lean (top), hip (middle top), ankle (middle bottom), and knee (bottom) angles in the army walking experiment. 

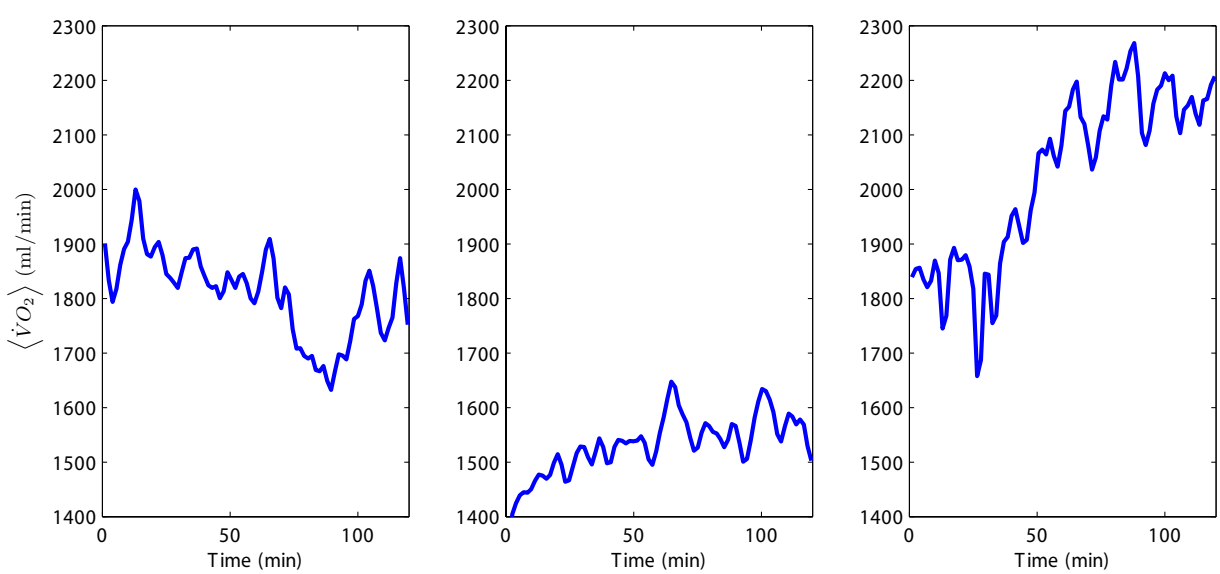

Figure 64. Estimated $\left\langle\dot{V} \mathrm{O}_{2}\right\rangle$ trends for subject 7 (left), 8 (middle), and 10 (right)
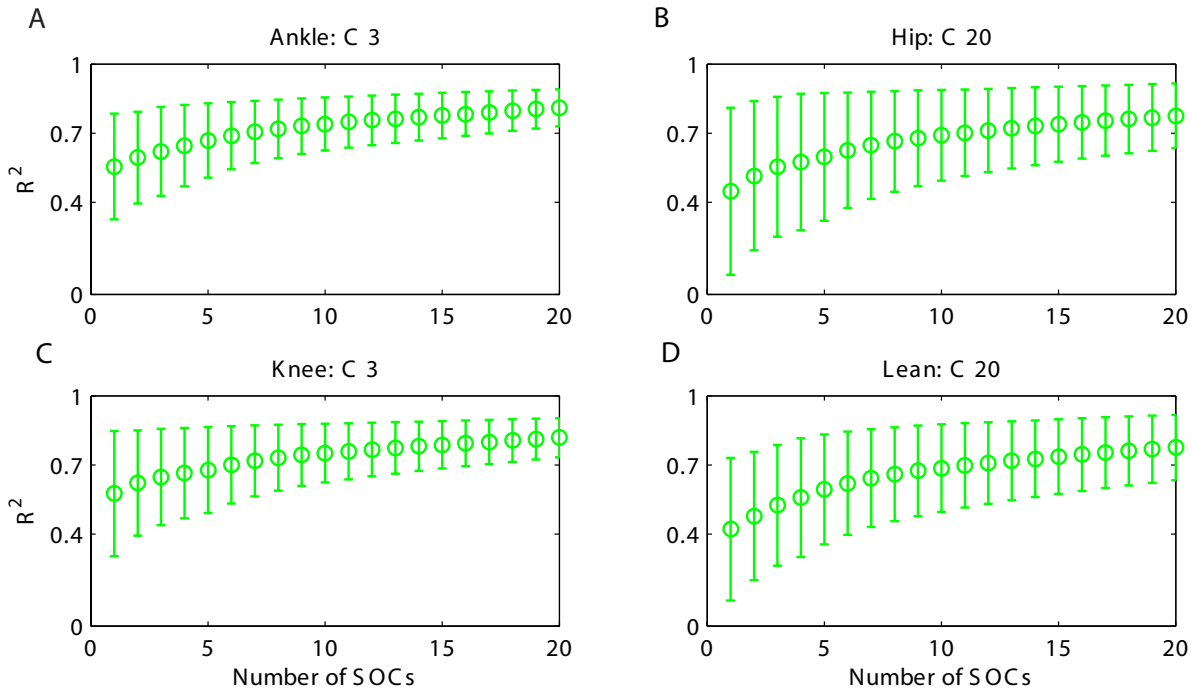

Figure 65. Cross subject variability in $R^{2}$ values for estimated $\left\langle\dot{V} O_{2}\right\rangle$ trends for cubic SOCs. Error bars represent one standard deviation from the mean of $R^{2}$ for (D: lean angle), (B: hip angle), (A: ankle angle), and (C: knee angle). Values on top of each plot indicate the number of SOCs needed to adequately track $\left\langle\dot{V} O_{2}\right\rangle$ trends as determined from the F-test. 

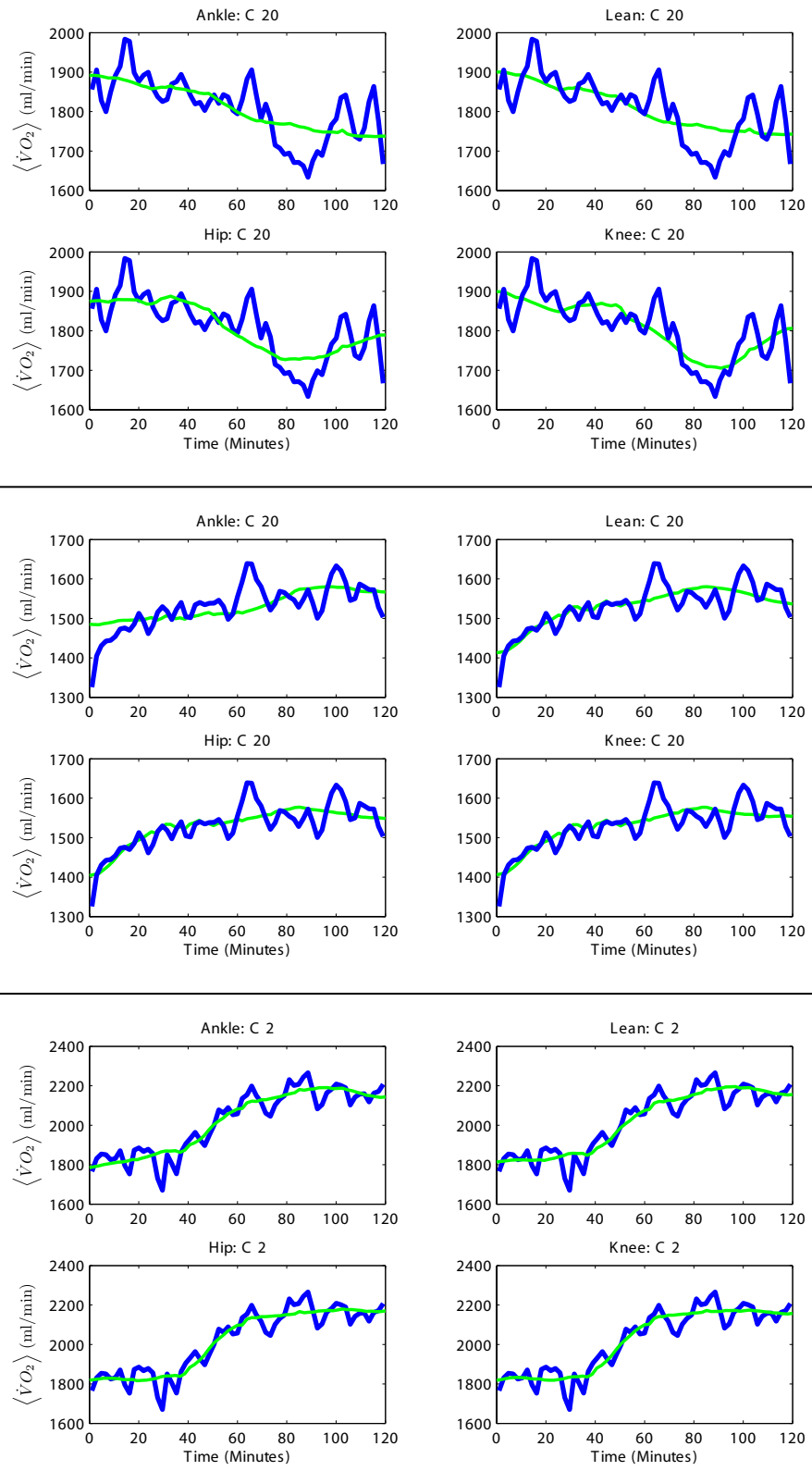

Figure 66. Linear combinations of nonlinear SOCs (green, thin - ) projected onto estimated $\left\langle\dot{V} O_{2}\right\rangle$ trends (blue, thick -) in a least squares sense for subject 7 (top), 8 (middle), and 10 (bottom). 


\subsection{Protein Dynamics}

The five residue peptide was simulated in the custom software GROMACS for $22 \mathrm{~ns}$ with an integration time step of $1 \mathrm{fs}$ with an applied constant pressure of 1 bar. All atoms were treated in the OPLS force field. The MD simulation with constraints ran for $2 \mathrm{~ns}$ at a temperature of $300 \mathrm{~K}$. Constraints were removed for the remaining $20 \mathrm{~ns}$. Position and velocity components were saved at every $0.25 \mathrm{ps}$ for all atoms in the backbone structure.

The equations of motion are known and can be simulated; therefore, the extended phase space is already embedded in the full MD model of the system. We can construct the auto covariance matrices directly from the recorded trajectories for each atom (excluding hydrogens) in the peptide. The position and velocity coordinates from all atoms are arranged into their own respective auto covariance matrices $\Sigma_{y y}$ and $\Sigma_{\dot{y} \dot{y}}$. Figures 67 and 68, depicts SOD-based analysis of $\Sigma_{y y}$ and $\Sigma_{\dot{y} \dot{y}}$ for simulated coordinates. Figure 67 shows the first 40 most dominant SOVs where we suspect the largest three dominant SOVs correspond to some manifestation of rigid body modes. The SOCs which correspond to the nine most dominant SOVs are shown in Fig. 68 and clearly the trends are saturated with high frequency content. By transforming the Cartesian coordinates into a set of internal coordinates the rigid body dynamics will not be captured.

Figures 69 and 70 depicts the results of the new auto covariance matrices for the transformed position and velocities coordinates into internal coordinates. Referring to Fig. 69, there are four separate groups of SOVs which are labeled on the plots as slow, faster, fast, and noise modes. The SOCs corresponding to the nine most dominant SOVs are depicted in Fig. 70. From the figure, the first five SOCs which correspond to the slow-time modes show the conformational transitions seen in Fig. 42. The SOCs corresponding to the faster modes show high 


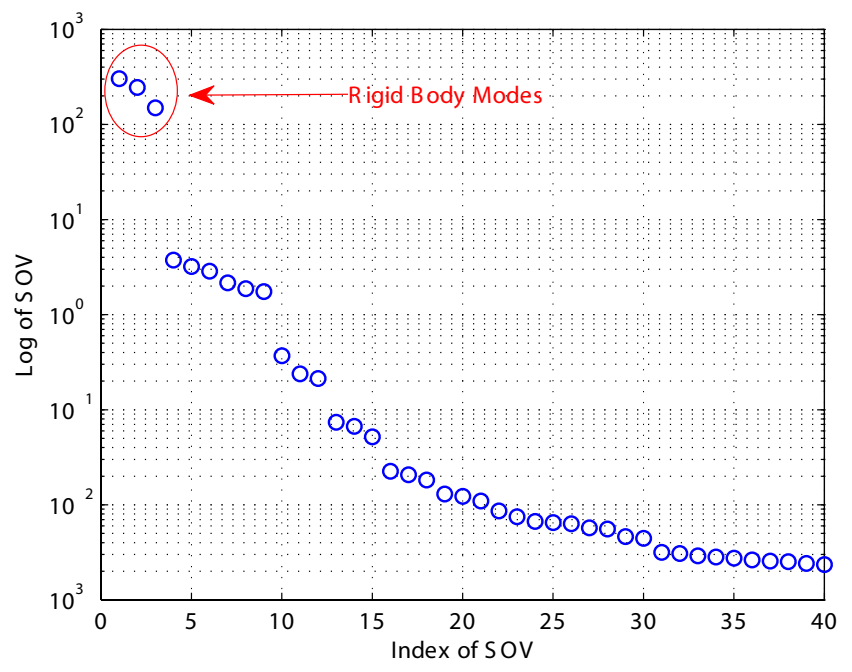

Figure 67. SOD analysis of cartesian coordinates from MD simulation. 40 dominant SOVs are depicted and the first three SOVs show some manifestation of rigid body modes.

frequency content which eventually leads to noise saturation.

Slow coordinate subspaces containing conformational dynamics can be obtained through the SOCs associated with the slow modes, as seen in Fig. 74. Likewise, the subspaces corresponding to the fast, faster, and noise modes are shown in Figs. 71, 72 and 73. Here, we lose the ability to uniquely differentiate the discrete islands representing the conformational states as the modes increase from faster to noise modes. Therefore, the conformational dynamics are limited to slow-time dynamics. Because of this distinct time scale separation of slow and fast dynamics, the modes pertaining to only the conformational dynamics can be sufficiently studied without the contamination of faster time scale dynamics.

It was claimed in [93] there are two conformational states the peptide can conform too. Figure 74 (left) shows two planes when plotting the first three SOCs against each other. However, in Fig. 74 (right) the figure is rotated and each plane is composed of many different groups of islands. It was hypothesized that each one of the islands is an actual conformation state. Therefore, there is not only 

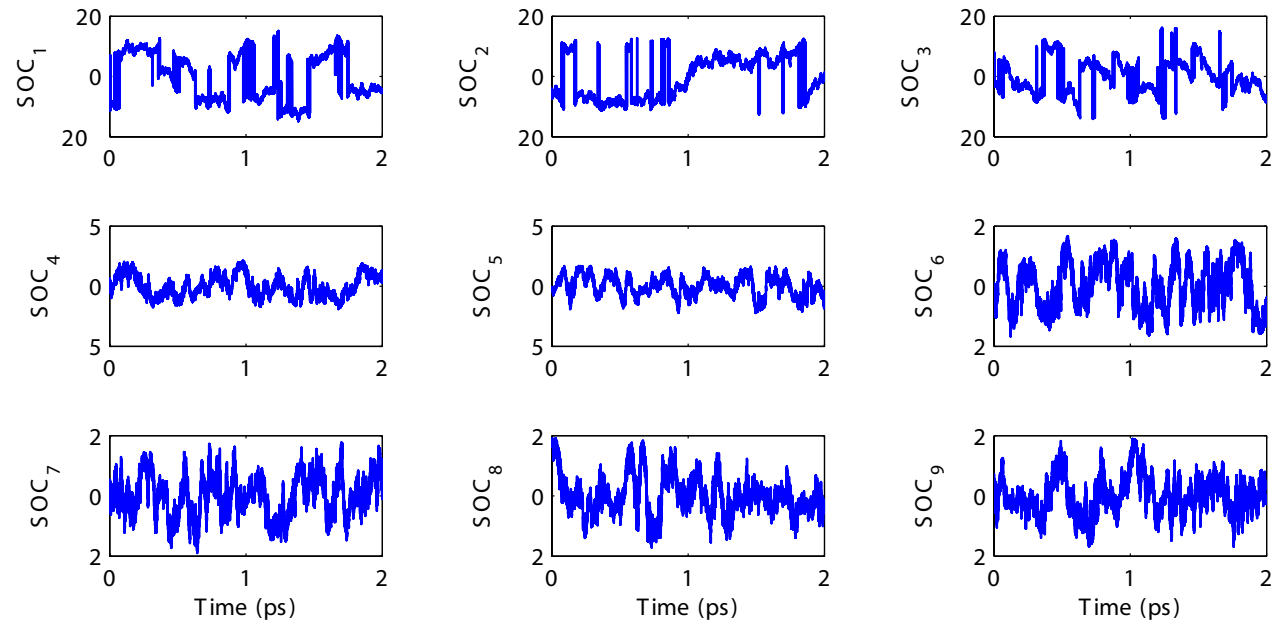

Figure 68. The first nine dominant SOCs are shown corresponding to the nine most dominant SOVs from the MD simulation. The SOCs are contaminated with high frequency content which could be a result of the rigid body motion.

two different conformational states - as seen in both the NMR experiment and numerical simulation - but 33 conformational states. The amount of time spent in each island is found by counting fractions of points that make up the particular island.

For this peptide Zhang et al. [1] performed an NMR experiment to determine the dihedral angles $\phi, \omega$, and $\psi$ for each residue in both conformational states, as seen in Table 3. Tables 4, 5, and 6 show the dihedral angles $\phi, \psi$, and $\omega$ for each slow island subspace. Each table is arranged in increasing order of the amount of time spent in that particular island. This value is shown in the right-most column of each table and values range from $0.107 \%$ to $11.478 \%$ of the time. The values calculated from the slow coordinate subspace do agree with the experimental values in Table 3 . In mostly all instances, the mean values are within the experimental value's standard deviation. This shows that not only does the slow subspace extract the two conformational states but finds an additional 33 conformational states that agree with experimental values. In addition, the amount of time spent in each conformation state is obtained. 


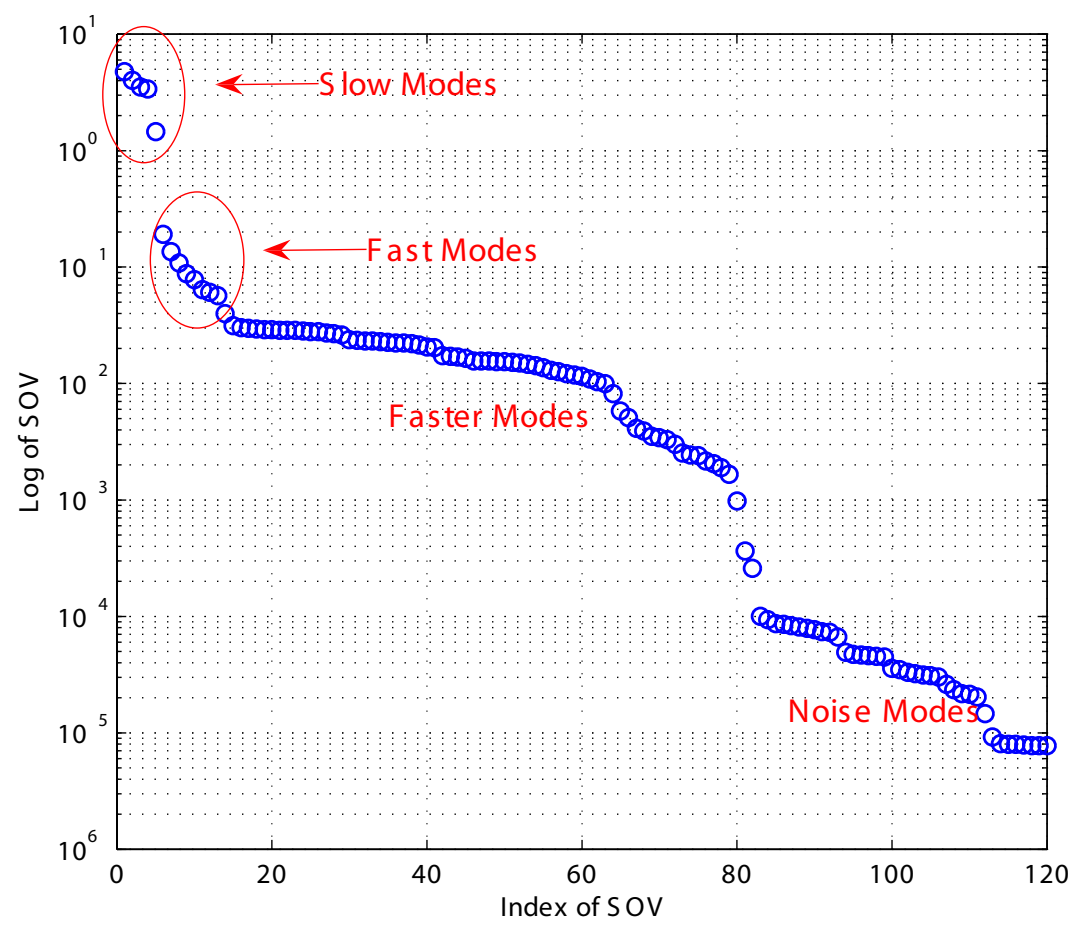

Figure 69. SOD analysis of generalized coordinates show four separated groups of SOVs corresponding to slow, faster, fast, and noise modes.
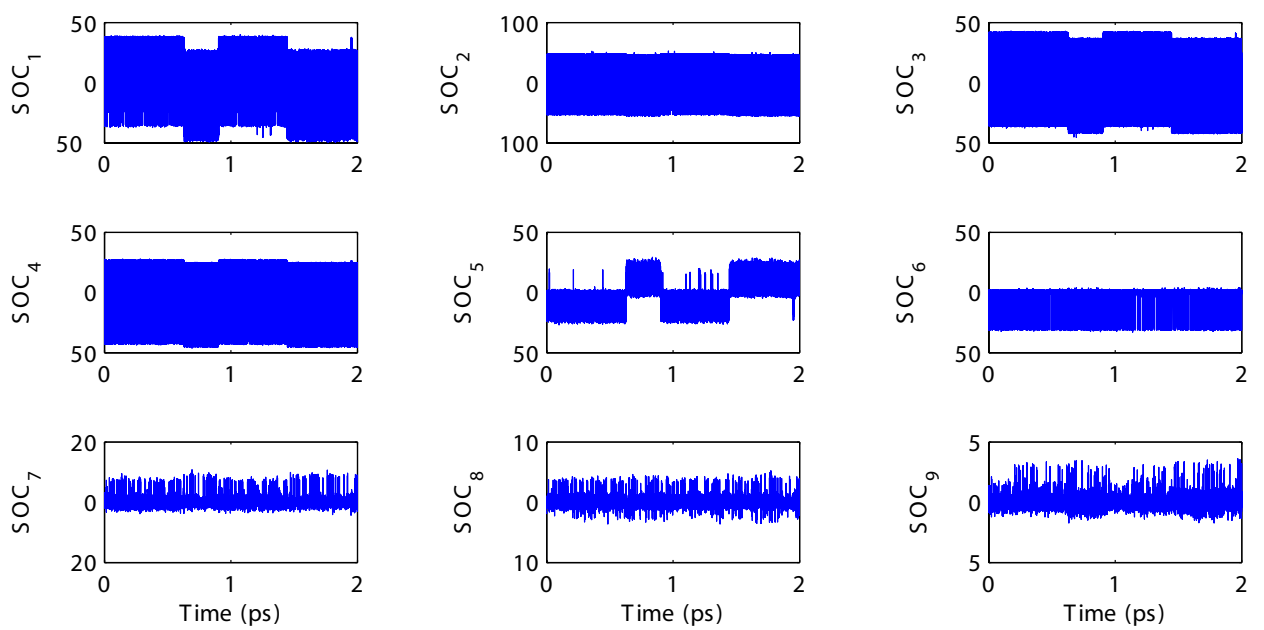

Figure 70. The first nine SOCs corresponding the nine most dominant SOVs. The first five dominant SOCs extract the flipping nature of the bond showing conformational changes. 


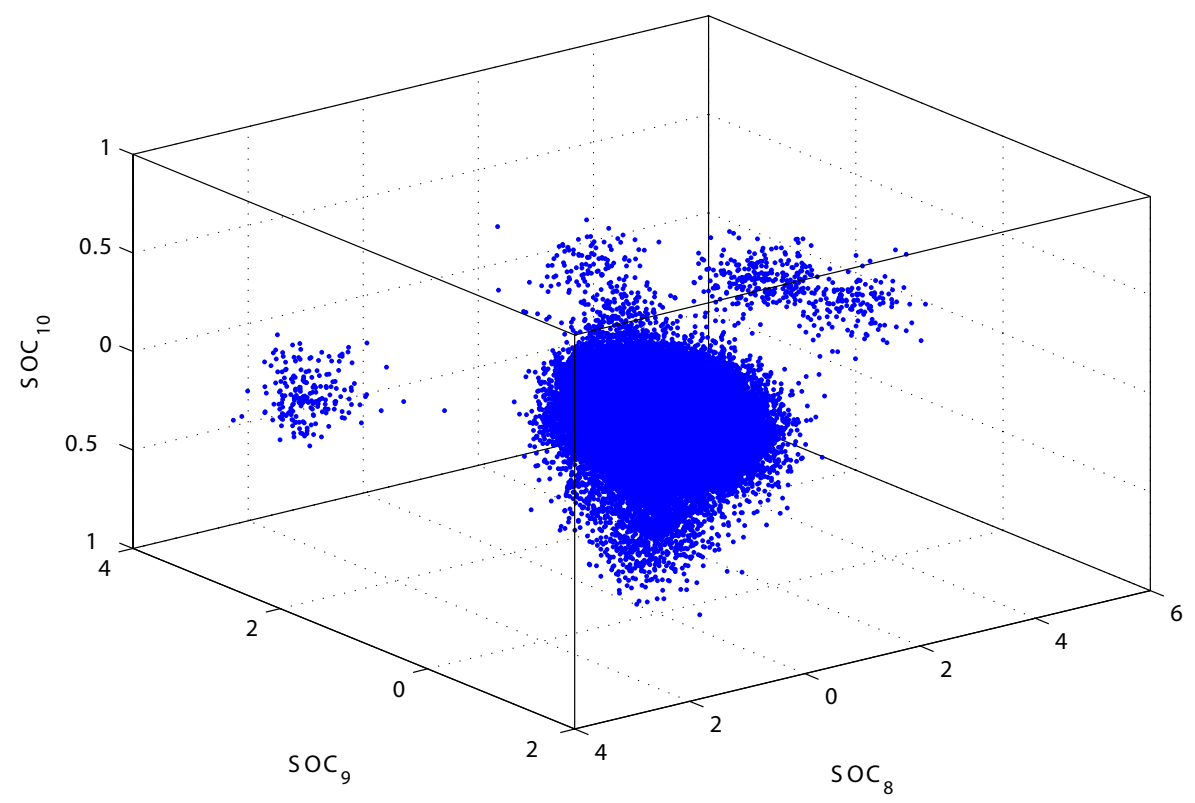

Figure 71. Faster coordinate subspace from SOD-based analysis of internal coordinates

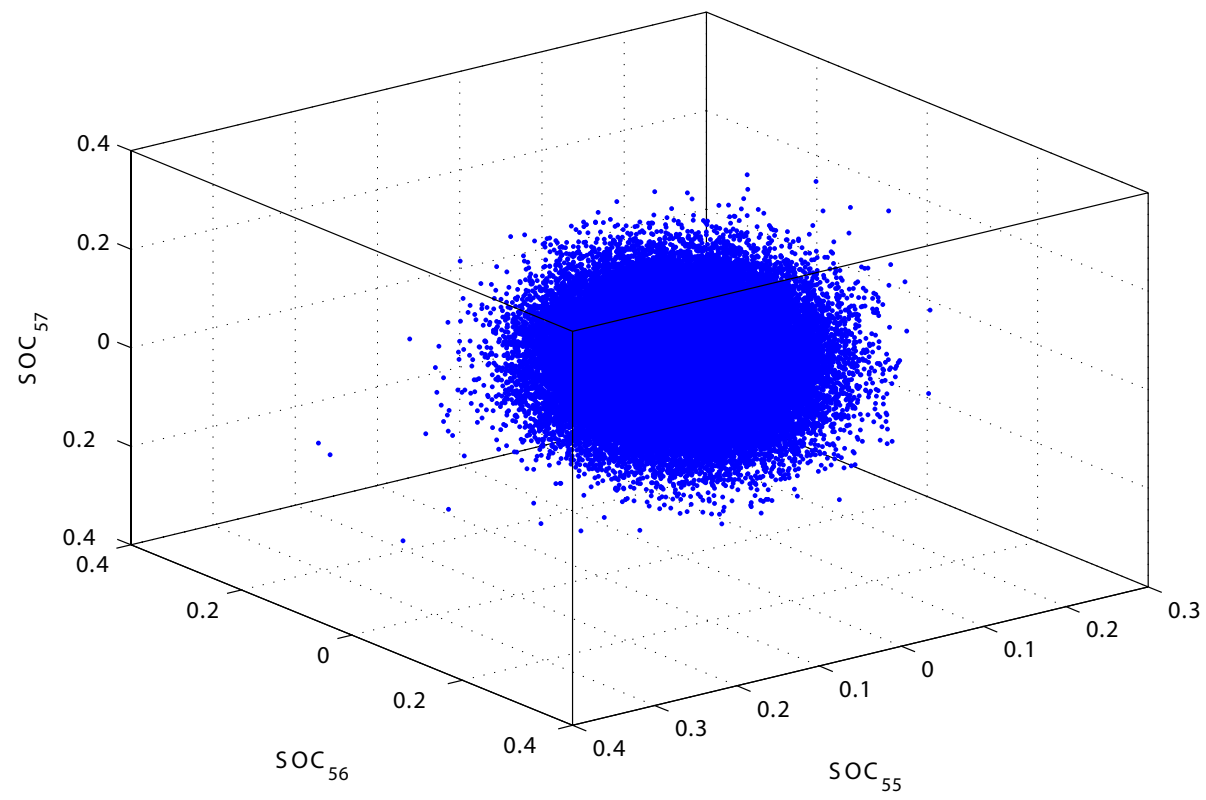

Figure 72. Fast coordinate subspace from SOD-based analysis of internal coordinates 


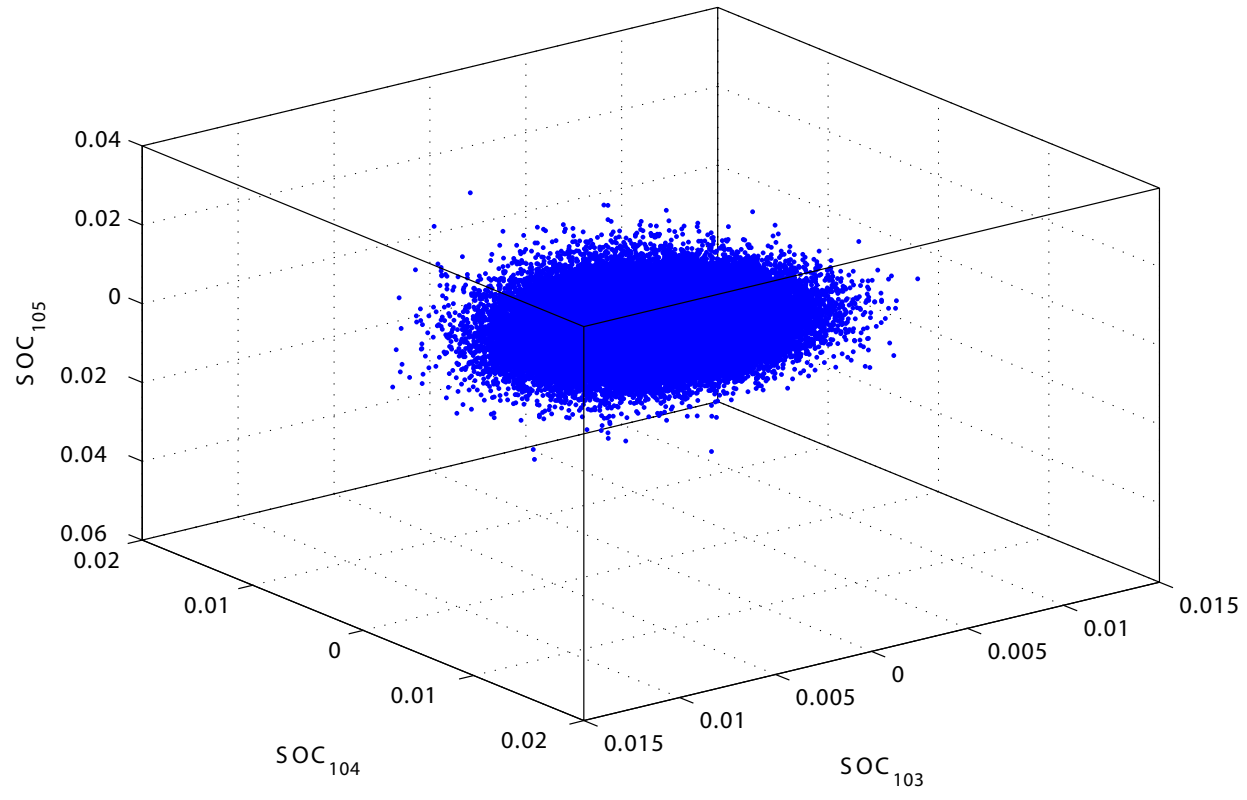

Figure 73. Noise coordinate subspace from SOD-based analysis of internal coordinates
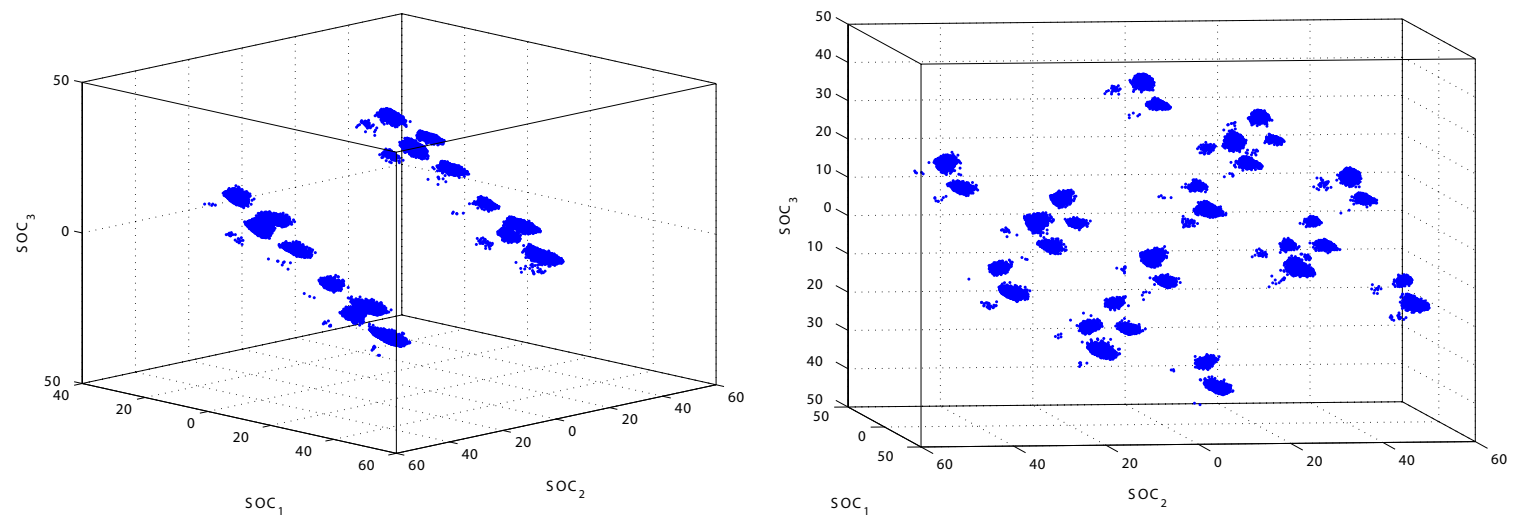

Figure 74. Slow coordinate subspace from SOD-based analysis of internal coordinates (Left) Two planes showing the two possible conformation states (Right) Rotation of the planes shows many discrete islands. 
Table 3. Dihedral angles (in degrees) for Conformers A and B [1]

\begin{tabular}{|c|c|c|c|}
\hline $\begin{array}{c}\text { Residue } \\
-\end{array}$ & $\begin{array}{c}\text { Angle } \\
-\end{array}$ & $\begin{array}{c}\text { Conformer A } \\
\text { Mean } \pm \text { SD }\end{array}$ & $\begin{array}{c}\text { Conformer B } \\
\text { Mean } \pm S D\end{array}$ \\
\hline${\mathrm{d}-\mathrm{Pro}^{1}}^{1}$ & $\phi_{1}$ & $67.8 \pm 9.6$ & $69.5 \pm 8.3$ \\
\hline- & $\psi_{1}$ & $-118.9 \pm 13.9$ & $-112.7 \pm 15.5$ \\
\hline- & $\omega_{12}$ & $176.5 \pm 6.2$ & $176.4 \pm 7.1$ \\
\hline $\mathrm{Ala}^{2}$ & $\phi_{2}$ & $-93.2 \pm 17.5$ & $-102.4 \pm 23.8$ \\
\hline- & $\psi_{2}$ & $8.3 \pm 19.0$ & $0.2 \pm 19.1$ \\
\hline- & $\omega_{23}$ & $177.3 \pm 8.8$ & $-179.9 \pm 8.8$ \\
\hline $\mathrm{Ala}^{3}$ & $\phi_{3}$ & $-126.5 \pm 22.1$ & $-145.5 \pm 16.1$ \\
\hline- & $\psi_{3}$ & $-119.3 \pm 31.8$ & $74.5 \pm 28.2$ \\
\hline- & $\omega_{34}$ & $173.2 \pm 7.4$ & $-176.9 \pm 6.7$ \\
\hline $\mathrm{Ala}^{4}$ & $\phi_{4}$ & $-85.9 \pm 32.9$ & $81.2 \pm 9.3$ \\
\hline- & $\psi_{4}$ & $-32.2 \pm 14.6$ & $-48.9 \pm 15.5$ \\
\hline- & $\omega_{45}$ & $159.0 \pm 7.8$ & $159.3 \pm 7.8$ \\
\hline $\mathrm{Ala}^{5}$ & $\phi_{5}$ & $-114.7 \pm 20.3$ & $-11.5 \pm 23.3$ \\
\hline- & $\psi_{5}$ & $145.1 \pm 11.4$ & $137.1 \pm 13.7$ \\
\hline- & $\omega_{51}$ & $-179.3 \pm 8.0$ & $-176.6 \pm 7.8$ \\
\hline
\end{tabular}




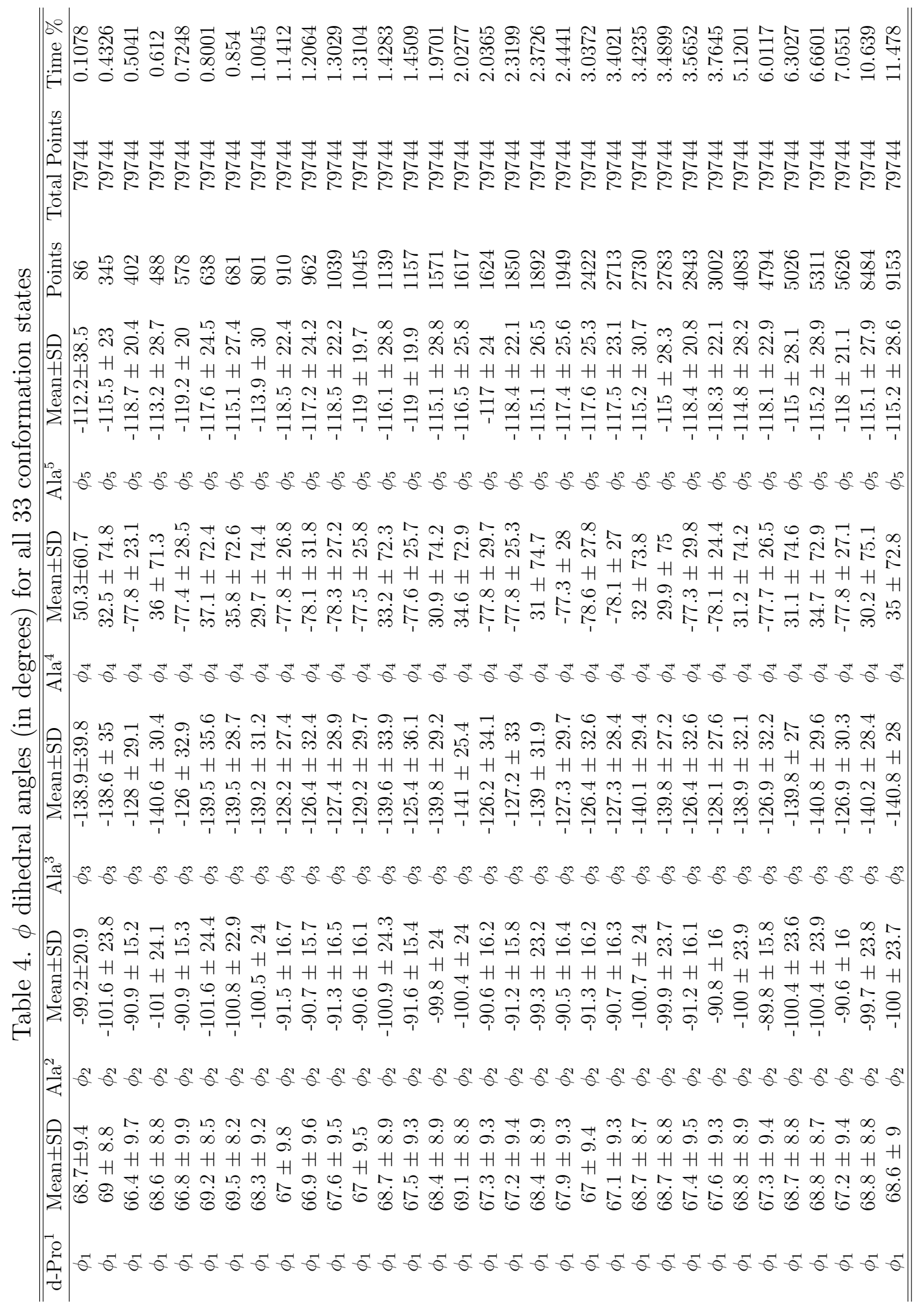




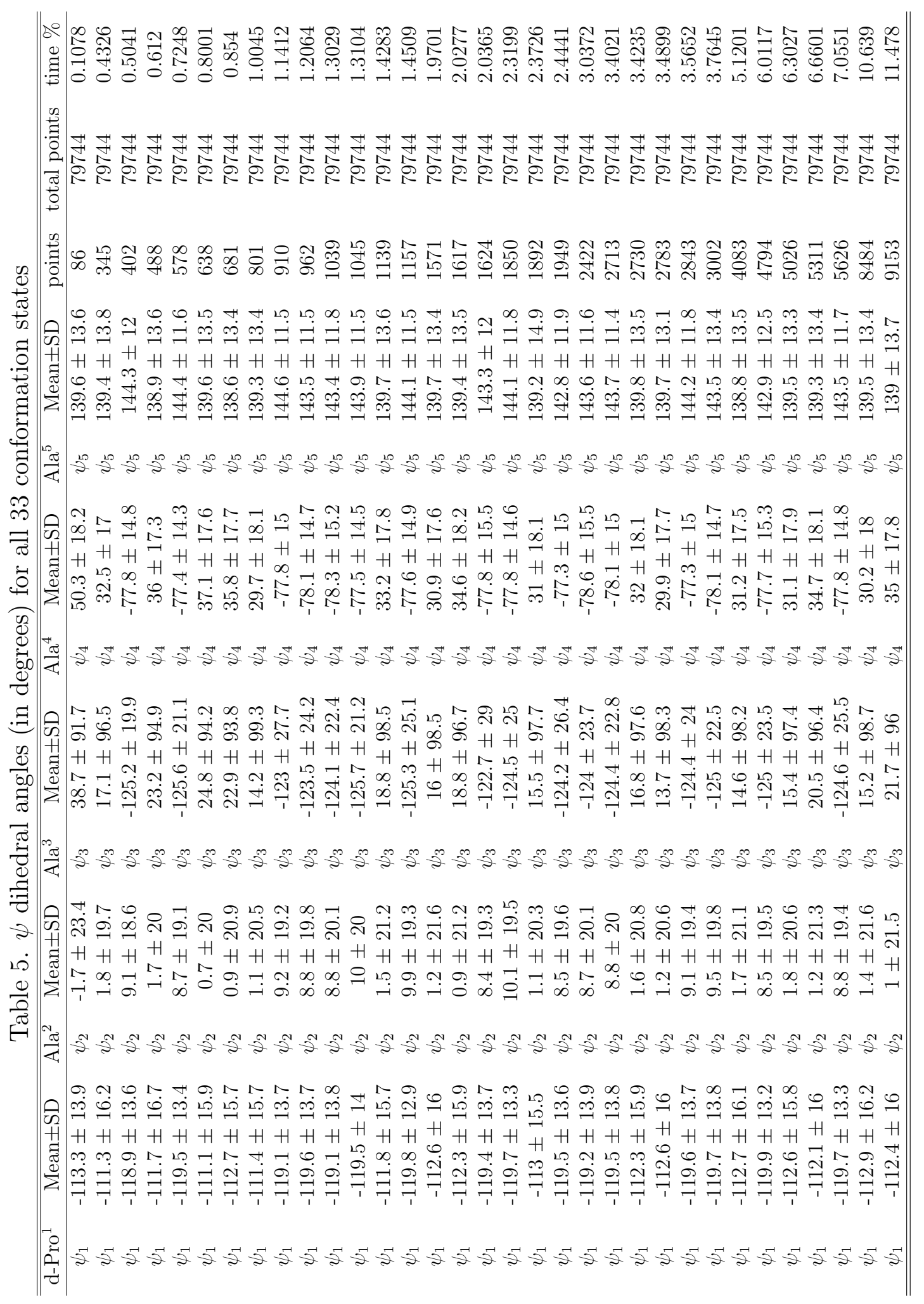




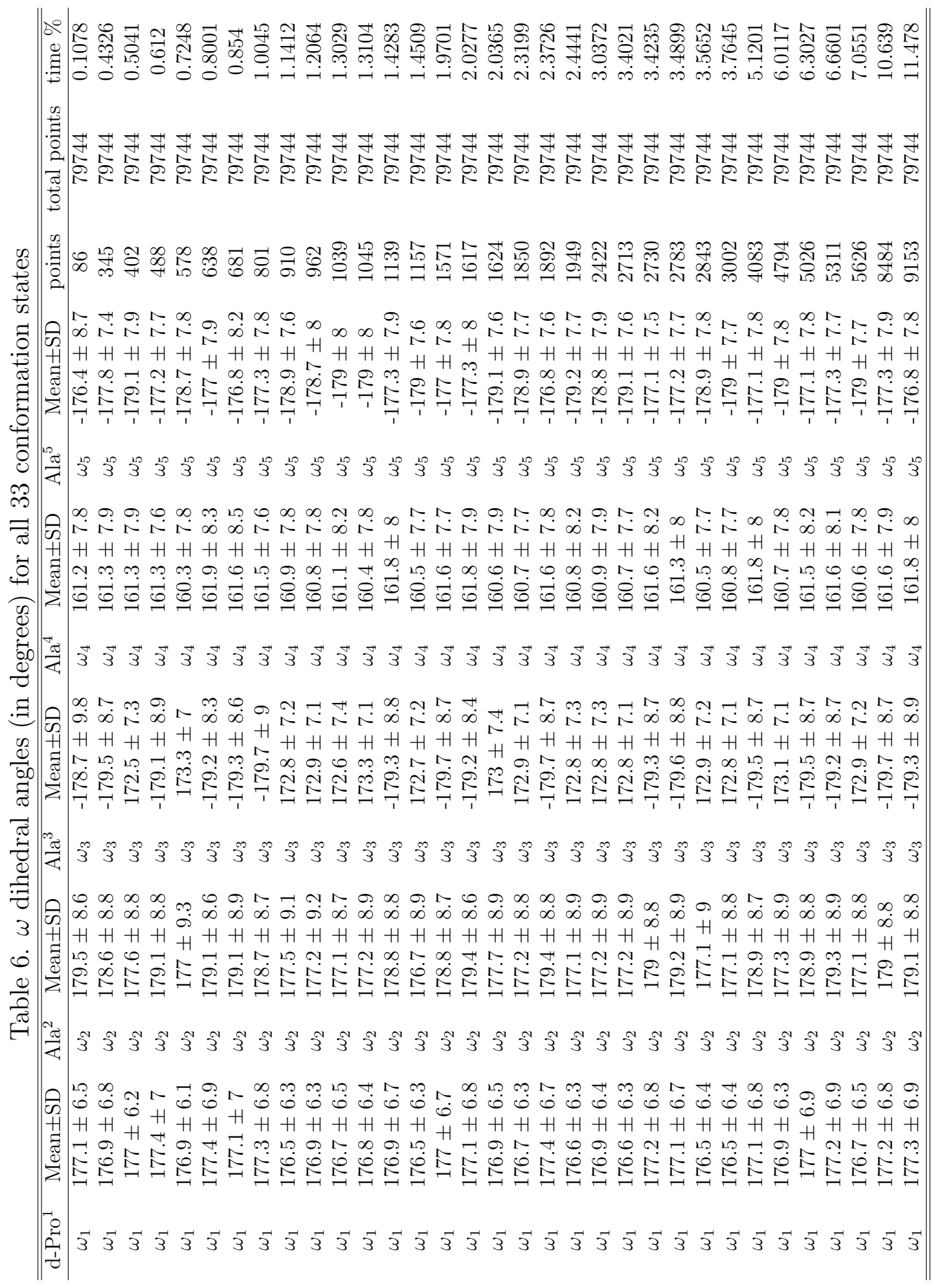




\section{CHAPTER 6}

\section{Discussion}

Smooth orthogonal decomposition, the newly developed multivariate data analysis method, is shown to separate signals according to their time scales. It was our intent to use these extracted signals to reconstruct the slow-time process from only the provided fast-time measurements. In reconstructing muscle fatigue, the fast-time measurements are recorded biomechanical variables. They are obtained from the subjects performing a sawing motion and from load carrying Army

soldiers. In the MD case, the fast-time measurements are the position and velocity coordinates from the molecular dynamic simulation of the peptide.

The effects that the STRMP weighting function for PSW-based features have on SOVs and SOCs are depicted in Fig. 47. The particular subject used in this plot is consistent with all other subjects and experiments. On average, using the weighting function results in a slight increase in the magnitude of SOVs. As a direct result, this produces slightly smoother coordinates. This would suggest that the weighting function provides an appropriate weight to the local linear model, and in turn, produces a more accurate tracking metric.

Figures 48, 53, and 58, depicts the energy in each SOD/POD mode and SOD/POD subspace for subject 6 in the sawing experiments and subject 10 in the walking experiments, respectively. Across all experiments, the same type of results are seen for the energy captured in each mode. POD - which is optimal in finding energy or signal power-captures more energy in roughly the first 15-20 modes in the sawing experiment and slightly fewer modes in the walking experiment. There is also a significant difference in the magnitude of the energy captured in the first several modes. On average, there is a minimum of two orders of mag- 
nitude difference in the energy in the modes. This is further seen in the percent of total energy each mode captures. Across all subjects in the sawing experiment, roughly $60 \%$ of the energy is captured in only the first two or three modes. For the walking soldiers, the ankle, knee, and hip angle captures $60-75 \%$ of the total energy in just the first mode.

Since SOD identifies time functions that are smoothest in time as possible, we see a jagged effect of the energy in the modes. This is because the modes are sorted with respect to smoothness and not only energy. But it is this smoothness that we are interested in, in tracking the fatigue. In Figs. 49, 54 and 59, the extracted coordinates from SOD and POD are compared. Clearly, there is a difference in the smoothness of the trends. The SOCs (red) are much smoother in time, however, POCs (blue) do track the global behavior of the trends. The SOCs and POCs start to approach each other as the index is increased to higher orders. Since fatigue is assumed to be a smooth deterministic process, energy alone is not a reliable measure in tracking fatigue in this context. Therefore, it is thought that the SOCs would capture the fatigue related trends in a lower dimensional subspace.

All experiments show similar and comparable results for the energy captured in each subspace. POD always finds slightly greater energy in each subspace when compared to SOD. In some instances, the values are very close to each other. This is seen until both trends start to level off at approximately a 20 dimensional subspace. At this point, POD and SOD find the same amount of energy in the subspace. However, this value is significantly decreased in the walking experiment.

In the sawing and walking experiments where the fast-time movement kinematics are used to try to reconstruct the slow-time process, one needs to take into consideration the temporal characteristics of the data. Above we compared the traditional tracking methodology of POD and found that energy would not pro- 
duce a robust measure of tracking fatigue from the PSW feature space. In other words, POD may not be suitable in extracting slow-time information since POD tries to find projections that maximize the variance or signal energy. The large amount of energy found by POD can be a result of bifurcations in the fast-time behavior. Any fast change in movement from one of the subjects can result in an instability causing a bifurcation in the fast-time dynamics which can contribute a significant amount of energy to the system. Using POD, the POCs will result in jumps due to these bifurcations. Therefore, SOD will produce a much better estimate of the smooth determinist fatigue trends than the traditional technique of POD since SOD is tailored to extract information about smooth deterministic processes.

SOD-based analysis for the high and low sawing experiments and walking experiment are depicted in Figs. 50, 55 and 60, respectively. The results for the linear (red, thick - ), quadratic (blue, thin --), and cubic (green, thin -) SOD are comparable across all angles and subjects. By increasing the order of SOD, the first SOV increases its magnitude. Again, suggesting that this produces smoother corresponding SOCs thus decreasing the local fluctuations of the SOCs.

Across all subjects, there is a much greater separation between the first linear and cubic SOV than between the first linear and quadratic SOV. Therefore, going from linear to quadratic SOD, the results are improved, but not drastically. By expanding the linear coordinates to a higher degree, the cubic SOVs are significantly greater then the linear SOVs. There is at least an order of magnitude difference between these values. This is further seen in the smoothness of the SOCs and the reduction in the number of coordinates needed to track the local and global fatigue dynamics.

These improvements in the nonlinear SOD are clearly seen in between subject 
variability $R^{2}$ values for the SOC based fits to the EMG markers, Figs. 51, 56 and 61. Two important aspects of Figs. 51, 56 and 61 give further validity to the nonlinear SOCs providing more optimal coordinates to describe fatigue dynamics. First, $R^{2}$ values from nonlinear SOCs are larger for all kinematic angles and muscle groups, cubic being the largest. This is an indication that for the same number of coordinates, nonlinear SOCs provide a better approximation to EMG-based trends. Even if the number of linear SOCs is increased substantially, nonlinear SOD based fits are still better. Second, there is a drastic decrease in the amount of SOCs that are needed to adequately track the EMG-based trends with the increase in the order of SOD.

The most drastic decrease in the number of SOCs needed to describe the fatigue dynamics is seen in the high sawing subjects, Fig. 51. For linear SOD, 20 SOCs are needed for adequate tracking in most of the angles followed by a slight decrease in quadratic and a significant decrease with cubic SOCs. For most of the angles and muscle groups, three to four cubic SOCs are needed, decreasing the dimensionality of the tracking SOC manifold by one order of magnitude. Even when only four or five linear SOCs are needed, the dimensionality is still decreased by at least one when using cubic SOCs. This is seen in the triceps muscle across all angles. Requiring so few SOCs to start, the triceps muscle may have experienced the most amount of fatigue and becomes a very reliable muscle to track overall fatigue. Muscles like the extensor carpi radialis need at least a 20 dimensional manifold to track the fatigue dynamics even when using nonlinear SOD. The fatigue may not have been tracked or it may not have experienced as much fatigue. Since the muscle is on the outer forearm, the muscle may be used to hold the handle more than provide the pushing and pulling force needed for the experiment.

Most of the same conclusions are drawn for the low sawing experiment, Fig. 56. 
The main difference is the amount of angles and muscle groups that show a significant decrease in the dimensionality of the tracking manifold. Again, the triceps muscle is reduced from a 20 dimensional manifold to a five dimensional manifold. An order-of-magnitude reduction is also seen in the pectoralis muscle for this experiment. For the low sawing motion, the pectoralis muscle could have been more directly effected by the motion and was needed more to complete this sawing motion. However, this muscle seemed to show few signs of fatigue in the high sawing experiment.

For the Army walking subjects, only the cubic SOCs are shown in Fig. 61. In all the muscle groups, only the right vastus medialis shows signs of improvement using cubic SOD. In addition, the cubic SOCs from the lean angle need only a four dimensional manifold to track the fatigue dynamics in the left tibialis fibulas. As will be seen in the $\dot{V} O_{2}$ and can be seen in the EMG trends, the results of subjects 7 and 8 shows that the SOD needs a very high dimensional space to capture the fatigue as indicated by $\dot{V} O_{2}$. Both subjects 7 and 8 started the experiment close to their $\dot{V} O_{2 \max }$ or exhausted very rapidly. Therefore, there was not a discernible trend to track as there was in subject 10 .

Global fatigue trend was expected to be evident from the $\dot{V} O_{2}$ data analysis. While subject 10 and subject 8 to lesser extent have shown the expected fatigue trend, subject 7 did not (Fig. 64). This is indicative of fixed task load level for subjects of different fitness levels. While subject 10 seems to have started substantially below his $\dot{V} O_{2 \max }$, subjects 7 and 8 were very close to it at the beginning of the task. By just these three subjects, using $\dot{V} O_{2}$ as a global fatigue indicator may not be robust enough. Figure 65 depicts the between subject variability $R^{2}$ values for the SOC based fits to the $\dot{V} O_{2}$ markers. For cubic SOD, the hip and lean angle still require at least 20 dimensions, whereas, the ankle and knee need a 
four dimensional manifold to track the global fatigue.

Figure 66 shows the nonlinear SOCs projected onto the $\dot{V} O_{2}$ trends in the least squares sense. Clearly, even 20 cubic coordinates in the projection are not able to track the $\dot{V} \mathrm{O}_{2}$ trends well for subjects 7 or 8 . However, only a two-dimensional manifold is needed for subject 10, Fig. 66 (bottom).

From Fig. 62, we can see that even using cubic SOCs, a 20 dimensional subspace is needed to adequately track the EMG trends. This can be attributed to two possible reasons. First, all of the kinematic measures were taken from the right side of the body and the EMG is recorded from both sides of the body. The results suggest that it would require a very high dimensional SOC subspace to reconstruct the local fatigue dynamics as indicated from the muscles on the left side of the body using the kinematics from the right side of the body. However, we still need a high dimensional subspace to reconstruct the local fatigue even on the right side of the body. Since the sawing experiment showed significantly better results, the walking data could have been contaminated with noise. Also, the experiments were conducted by different experimentalists in different labs using different equipment. Secondly, the right and left side of the body moves asymmetrically. This is seen in a few muscles where there is an order-of-magnitude difference. For instance, this is seen in the vastus medialis, gastrocnemius, and tibialis femoris muscles. With these reasons we can speculate about some of the discrepancy in the results.

Figures 52, 57, and 63 shows both linear and nonlinear coordinates projected onto EMG trends for subjects 6 and 10 in the sawing and army experiments, respectively. The amount of SOCs in each projection are indicated on top of the corresponding plots. The results suggest that the cubic SOCs track the EMG trends with more precision and are closer to the actual trends. The linear SOC projections contain more high frequency information than the nonlinear, with cubic 
being the smoothest projection.

A comparison of both the high and low sawing experiment further validates the ability of the PSW/SOD methodology. In each exercise, there are predominant muscles that are increasingly tasked with each sawing motion. These muscles should show greater signs of fatigue, and therefore, should need a lower-dimensional manifold to track. The major group of muscles that are shared between both experiments is the anterior, middle, and posterior deltoid muscle. This muscle should be fatigued more rapidly and more severely in the high sawing experiment. In the low sawing experiment, although this group of muscles are not the primary muscles involved they should show some signs of fatigue.

From a physiological standpoint, the SOD-based analysis agree with the deltoid muscles, in both sawing experiments. In the between-subject-variability $R^{2}$ values for the high sawing experiment (see Fig. 51), all three deltoid muscles show a drastic reduction in the dimensionality of the tracking manifold needed with an increasing order of SOD. Except for the humeral plane angle for the posterior deltoid, only three to five cubic SOCs are needed to track the fatigue dynamics. This suggest that with more fatigue present, cubic SOD can track the fatigue dynamics in a lower-dimensional space. In the low sawing experiment, the posterior deltoid in the elbow flexion/extension and humeral plane angle need a four- and five-dimensional manifold to track, respectively. The anterior and middle deltoid in all three angles still need 20 cubic SOCs. This is a result of the level of fatigue to track and not the method of doing so.

For the molecular dynamics results, the SOD-based analysis of the Cartesian coordinates are shown in Figs. 67-68. In figure Fig. 67, there are a set of three modes that are significantly greater than the rest of the modes. It is believed that these modes are a manifestation of rigid body modes. The corresponding coordi- 
nates are contaminated with high-frequency noise and there is no clear discernible trend in the coordinates. However, by transforming the coordinates to generalized or internal coordinates, this rigid body motion can be eliminated.

The SOD-based analysis of the generalized coordinates are shown in Figs. 6970. The first thing to notice is the four different clusters of points using internal coordinates where the first cluster of points is believed to be slow-time modes. The other groups are labeled as fast, faster, and noise modes. Figure 70 shows that SOD is able to extract the flipping nature of the peptide between conformational states from only the fast-time measurements. The first five modes that show this trend correspond to the slow-time modes in Fig. 69. Therefore, when comparing to Fig. 42, provided only the position and velocities, SOD is able to extract the slow-time information and in doing so, extract conformational transitions for the two conformational states.

It was hypothesized that the slow-time modes extracted from SOD will contain the amount of time the peptide stays in each conformational state. In doing so, the slow-time subspace shows not only two conformational states as assumed by [93], but 33 conformational states. Figure 74 shows the slow-time subspace and in Fig. 74 (left), there are two planes which correspond to the two conformational states. However, each plane is composed of several clusters of points or 33 discrete islands, Fig. 74 (right). Since only the two conformational states were seen by the MD simulation and even by NMR, the conformational dynamics are obscured by the fast-time dynamics. However, SOD analysis shows that there are hidden dynamics that are obscured since 31 additional conformational states were found.

It is thought that the amount of points in each island is the percentage of time the peptide stays in that conformation. Tables 4,5 , and 6 show the dihedral angles $\phi, \psi$, and $\omega$ for each slow island subspace. The time spent in that particular island 
range from $0.107 \%$ to $11.478 \%$ of the total time. The amount of time is compared to the values in Table 3 . The values in the table are only for the two conformational states but the values calculated in this work are very similar. Most of the values are within the standard deviation.

The SOD-based analysis of the short-time simulation shows the ability to extract the conformational transitions as seen by NMR experiments. Since a limiting factor of MD simulations is the storage of large amounts of data, the short-time MD simulation is quite valuable. By requiring less storage space, large peptides or proteins can be simulated for a short amount of time. The slow-time subspace also identifies additional conformation states and the time the peptide stays in each conformation. By having this information, a transition map between conformations can be acquired. 


\section{CHAPTER 7}

\section{Conclusion}

In complex systems with drastic time scales, fast-time scales may dominate and/or obscure slow-time processes. Current methodologies are limited in their ability to use fast-time dynamics to infer slow-time processes. The objective of this dissertation was to use the newly developed multivariate data analysis method of smooth orthogonal decomposition to extract slow-time dynamics from only fasttime data. This method was applied to two different hierarchical dynamical systems which possesses couplings between their slow-time and fast-time dynamics. The two different applications were: (1) biomechanical motion kinematics are used to identify physiologic muscle fatigue processes in humans, and (2) a short-time simulation of a peptide using a full scale molecular dynamics (MD) model is used to identify slow-time dynamic modes and possible conformational states.

It was hypothesized that: (1) SOD analysis of measured fast-time motion kinematics can be used to reconstruct the slow-time dynamics of muscle fatigue to establish a mapping between the kinematics and fatigue and (2) a nonlinear extension of SOD can identify more optimal fatigue coordinates to provide a lowerdimensional reconstruction of the fatigue dynamics. These were demonstrated through two different experiments. First, ten healthy right-handed subjects performed a sawing motion by pushing a weighted handle back and forth until voluntary exhaustion. This task was performed with the subject's arm in a low and high position. Three sets of joint kinematic angles were measured from the elbow, wrist and shoulder as well as surface Electromyography (EMG) from nine different muscle groups.

For the second experiment, three healthy load carrying U.S Army soldiers 
walked continuously on a treadmill. In addition to measuring joint kinematic of the lean, ankle, hip, and knee angles and EMG from ten muscle groups, breathby-breath oxygen consumption $\left(\dot{V} \mathrm{O}_{2}\right)$ were measured too. It was shown that SOD produces better estimates then the traditional tracking methodology of POD. Since POD only tries to maximize the variance, the method is not optimal to extract smooth slow-time trajectories. In addition, noisy PSW feature vectors may cause jumps in the POCs due to bifurcations in the fast-time movement kinematics. Therefore, it was found that SOCs can better estimate the slow-time trajectories than conventional methods. In the feature vector estimation, a weighting function was applied to the local linear model. Results showed that on average, the weighting function provided a better tracking function. Adapting the new weighting model, SOD analysis indicates that the linear SOCs were contaminated with more high-frequency content than the corresponding nonlinear coordinates for the same modal index. This resulted in a significant reduction of the number nonlinear SOCs need to reconstruct both the local and global muscle fatigue dynamics when compared to linear SOCs.

Between subject variability coefficient of determination $R^{2}$ values indicate roughly a 20-dimensional manifold using linear SOD analysis is needed to reconstruct the traditional EMG-based local muscle fatigue and $\dot{V} O_{2}$-based global whole body fatigue indicators. It was also found that approximately a 20-dimensional subspace would be needed to capture a significant amount of energy in the data. This paralleled results from using POD which is optimal in finding the energy in a signal. However, utilizing nonlinear SOD, an order-of-magnitude reduction in the number of SOCs was seen with a four- or five-dimensional manifold capturing the same fatigue dynamics with quadratic SOCs. Furthermore, in some instances, only a two-dimensional manifold was needed with cubic SOCs. Thus, nonlinear 
SOD extracts more optimal coordinates than linear SOD, and in doing so, reduces the required dimensionality by roughly one order of magnitude.

For the molecular dynamics, it would be advantageous to run a short simulation for a few nanoseconds to identify appropriate time scale modes to simulate the long time dynamics. It was hypothesized that: (1) SOD analysis of a full scale short time simulation can identify slow-time modes and (2) slow coordinate subspaces will contain the peptide conformational geometry and information about the amount of time the peptide stays in each conformation. This was demonstrated by simulating a five reside peptide for a short time period. Atomic trajectories were obtained over the entire simulation and transformed into internal coordinates. SOD analysis of internal coordinates indicate a group of 5 slow-time modes. In addition, SOD identified slow-time coordinates which show the conformational transitions between the two assumed conformational states.

The slow-time subspace shows 33 discrete clusters of points which is thought to represent 33 conformational states. Therefore, SOD is able to indicate 31 additional conformational states which traditional NMR experiments were not able to do in this case. Furthermore, the number of points in each discrete cluster indicated the amount of time the peptide stays in that particular conformation. The amount time spent in each conformational state ranges from $0.107 \%$ to $11.478 \%$ of the total time. From here, a transitional map between conformations can be acquired from only fast-time simulation data.

\subsection{Future Work}

There are three recommendations from this author for future work. A new global fatigue marker is needed. $\dot{V} O_{2}$ was shown to be a good indicator of global fatigue in only one out of three subjects. A different marker such as lactic acid may provide to be a more reliable measure. For SOD, the column space has to be 
less than the row space of the matrix. Therefore, the number of modes retained for nonlinear SOD can not exceed a certain value, which can limit the results. Considerable attention is needed to devise a criterion or method to determine the number of modes to be retained for nonlinear SOD. In the molecular dynamics, a small peptide should be simulated for a short-time. A reduced order model should be developed and simulated to see if the dynamics of the peptide can be recovered in a much lower-dimensional space.

Lastly, the authors would like to acknowledge a mistake in the weighting function. As the formula stands now, if the cloud of nearest neighbors approaches a circle, then there is more weight. However, it should be that when the cloud becomes a circle, the orientation is weighted less. The reason is that when the cloud is a circle, there is no significant degree of elongation of the cloud, and then the orientation of the cloud along the STRMP error is irrelevant. This could be why there was only a slight improvement in the results using this weight. Further consideration is needed in address the weighting function. 


\section{LIST OF REFERENCES}

[1] X. Zhang, G. Nikiforovich, and M. G.R, "Conformational templates for rational drug design: Flexibility of cyclo(d-pro1-ala2-ala3-ala4-ala5) in dmso solution," Journal of Medicinal Chemistry, vol. 50, pp. 2921-2925, 2007.

[2] Southeastern univeristy and college coalition for engineering eduction, Lecture 19. Tissues, Organs, Protection, Support, and Movement, UNIT 4. ANIMAL SYSTEMS. [Online]. Available: http://www.succeed.ufl.edu/ content/abe2062/lect/lect_19/lect_19.htm

[3] Department of Biology, Biosciences Web Site: www.nvo.com/jin, 1998-2010. [Online]. Available: http://www.nvo.com/jin/homepage1/

[4] S. Doebling, C. Farrar, M. Prime, and D. Shevitz, "Damage identification and health monitoring of structural and mechanical systems from changes in their vibration characteristics: A literature review." Tech. Rep. LA-13070-MS, Los Alamos National Laboratory, Los Almos, New Mexico 87545., Tech. Rep., 1996.

[5] S. Güttler, H. Kantz, and E. Olbrich, "Reconstruction of the parameter spaces of dynamical systems," The American Physical Society, vol. 63, p. 056215, 2001.

[6] M. Song, D. B. Segala, J. B. Dingwell, and D. Chelidze, "Slow-time changes in human muscle fatigue are fully represented in movement kinematics," Journal of Biomechanical Engineering, vol. 131, pp. 021 004(1-11), 2009.

[7] D. Chelidze, J. Cusumano, and A. Chatterjee, "Dynamical systems approach to damage evalution tracking, part 1: desccription and experimental application," Journal of Vibration and Acoutics, vol. 124, no. 2, pp. 250-257, 2002.

[8] D. Chelidze and J. P. Cusumano, "Phase space warping: nonlinear time series analysis for slowly drifting systems," Philosophical Transactions of the Royal Society A, vol. 364, pp. 2495-2513, 2006.

[9] P. Verdes, H. Granitto, Pand Navone, and H. Ceccatto, "Nonstationary timeseries analysis: accurate reconstruction of driving forces," Phys. Rev. Lett, vol. 87(12), p. 124101(4), 2001.

[10] P. Verdes, P. Granitto, and H. Ceccatto, "Overembedding method for modeling nonstationary systems," Phys. Rev. Lett, vol. 96(11), p. 118701(4), 2006. 
[11] J. Cusumano, D. Chelidze, and A. Chatterjee, "Dynamical systems approach to damage evalution tracking, part 2: model-based validation and physical interpretation," Journal of Vibration and Acoutics, vol. 124, no. 2, pp. 258264, 2002.

[12] D. Chelidze and M. Liu, "Dynamical systems approach to fatigue damage identification," Journal of Sound and Vibration, vol. 281, pp. 887-904, 2005.

[13] D. Chelidze and M. Liu, "Reconstructing slow-time dynamics from fast-time measurements," Philosophical Transaction of the Royal Society A, vol. 366, pp. 729-3087, 2008.

[14] D. Chelidze, "Identifying multidimensional damage in a hierarchical dynamical system," Nonlinear Dynamics, vol. 31, pp. 307-322, 2004.

[15] J. B. Dingwell, D. F. Napolitano, and D. Chelidze, "A nonlinear approach to tracking slow-time-scale changes in movement kinematics," Journal of Biomechanics, vol. 40, pp. 1629-1634, 2006.

[16] Y. C. Liang, H. P. Lee, S. P. Lim, W. Z. Lin, K. H. Lee, and C. G. Wu, "Proper orthogonal decomposition and its applications-part i: Theory," Journal of Sound and Vibration, vol. 252, pp. 527-544, 2002.

[17] A. Chatterjee, "An introduction to the proper orthogonal decomposition," Current Science, vol. 78, no. 7, pp. 808-817, 2000.

[18] S. Volkwein, "Model reduction using proper orthogonal decomposition. lecture notes,", Institute of Mathematics and Scientific Computing, University of Graz, Tech. Rep., 2008.

[19] B. F. Feeny and R. Kappagantu, "On the physical interpretation of proper orthogonal modes in vibrations," Journal of Sound and Vibration, vol. 211(4), pp. 607-616, 1998.

[20] W. Zhou and D. Chelidze, "Blind source seperation based vibration mode identification," Mechanical Systems and Signal Processing, vol. 21, pp. 30723087, 2007.

[21] G. Kershen, P. F, and J. Golinval, "Physical interpretation of independent component analysis in structural dynamics," Mechanical Systems and Signal Processing, vol. 21, pp. 1567-1575, 2007.

[22] A. Vakakis, "Non-linear normal modes (nnms) and their application in vibration theory: An overview," Mechanical Systems and Signal Processing, vol. 11(1), pp. 3-22, 1997.

[23] S. Shaw and C. Pierre, "Non-linear normal modes and invariant manifolds," Journal of Sound and Vibration, vol. 150(1), pp. 170-173, 1991. 
[24] S. Shaw and C. Pierre, "Normal modes for non-linear vibratory systems," Journal of Sound and Vibration, vol. 164(1), pp. 85-124, 1993.

[25] S. Shaw and C. Pierre, "Normal modes of vibration for non-linear continuous systems," Journal of Sound and Vibration, vol. 169(3), pp. 319-347, 1994.

[26] L. Yang, E. Eyal, I. Bahar, and A. Kitao, "Principal component analysis of native ensembles of biomolecular structures (pca nest): insights into functional dynamics," Bioinformatics (Oxford, England), vol. 25, pp. 606-614, 2009.

[27] G. Maisuradze, A. Liwo, and H. Scheraga, "Principle component analysis for protein folding dynamics," Journal of Molecular Biology, vol. 385, pp. 312$329,2009$.

[28] F. Lange and H. Grubmuller, "Can principle components yield a dimension reduction desccription of protein dynamics on long time scales," Journal of Chemical Physics B, vol. 110, pp. 22 842-22 852, 2006.

[29] J. Shlens, "A tutorial on principal component analysis," Institute for nonlinear science, University of California, San Diego, La Jolla, CA 92093, Tech. Rep., 2005 .

[30] G. Berkooz, P. Holmes, and L. Lumley, Jonh, "The proper orthogonal decomposition in the analysis of turbulent flows," Annuals Review of Fluid Mechanics, vol. 25, pp. 539-575, 1993.

[31] B. Feeny, "On the proper orthogonal modes and normal modes of a continuous vibration system," Journal of Sound and Vibration, vol. 124(1), pp. 157-160, 2002.

[32] D. Chelidze and W. Zhou, "Smooth orthogonal decomposition based modal analysis," Journal of Sound and Vibration, vol. 292, no. 3-5, pp. 461-473, 2006.

[33] U. Farooq and B. Feeny, "Smooth orthogonal decomposition for modal analysis of ramdomly excited systems," Journal of Sound and Vibration, vol. 316, pp. 137-146, 2008.

[34] B. Bigland-Ritchie and J. J. Woods, "Changes in muscle contractile properties and neural control during human muscular fatigue," Muscle $\&$ Nerve, vol. 11, pp. 251-279, 1984.

[35] C. J. DeLuca, "Myoelectrical manifestations of localized muscular fatigue in humans," Critical Reviews in Biomedical Engineering, vol. 11, pp. 251-279, 1984.

[36] S. C. Gandevia, "Spinal and supraspinal factors in human muscle fatigue," Physiological Reviews, vol. 81, pp. 1725-1789, 2001. 
[37] D. Allen, J. Lannergren, and H. Westerbald, "Limits to human performance caused by muscle fatigue," Journal of Physiology and Experimental Physiology, pp. 7-10.

[38] F. H. Martini, M. J. Timmons, and R. B. Tallitsch, Human Anatomy. Prentice-Hall Inc., 1995.

[39] L. Geddes, "Superhuman," New Scientist, vol. 07-28, pp. 35-41, 2007.

[40] J. N. Ct, P. A. Mathieu, M. F. Levin, and A. G. Feldman, "Movement reorganization to compensate for fatigue during sawing," Experimental Brain Research, vol. 146, no. 3, pp. 394-398, 2002.

[41] D. D. Ebaugh, P. W. McClure, and A. R. Karduna, "Effects of shoulder muscle fatigue caused by repetitive overhead activities on scapulothoracic and glenohumeral kinematics," Journal of Electromyography and Kinesiology, vol. 16, no. 3, pp. 224-235, 2006.

[42] M. L. Madigan and P. E. Pidcoe, "Changes in landing biomechanics during a fatiguing landing activity," Journal of Electromyography and Kinesiology, vol. 13, no. 5, pp. 491-198, 2003.

[43] J. Mizrahi, O. Verbitsky, E. Isakov, and D. Daily, "Effect of fatigue on leg kinematics and impact acceleration in long distance running," Human Movement Science, vol. 19, no. 2, pp. 139-151, 2000.

[44] P. Sparto, M. Parnianpour, T. Reinsel, and S. Simon, "The effect of fatigue on multijoint kinematics, coordination, and postural stability during a repetitive lifting test," Journal of Orthopaedic E3 Sports Physical Therapy, vol. 25, no. 1, pp. 3-12, 1997.

[45] K. Voge and J. Dingwell, "Relative timing of changes in muscle fatigue and movement coordination during a repetitive one-hand lifting task," in Proceedings of the 25th Annual International Conference of the IEEE Engineering in Medicine and Biology Society. Cancun, Mexico: Institute of Electrical and Electronics Engineers, 2003, pp. 1807-1810.

[46] P. Frykman, E. Harman, J. Knapik, and K. Han, "Backpack vs. front pack: Differential effects of fatigue on loaded walking posture (abstract only)," Med Sci Sports Exerc, vol. 26, p. 140, 1994.

[47] J. Knapik, E. Harman, and K. Reynolds, "Load carriage using packs: A review of physiological, biomechanical and medical aspects," Appl Ergon, vol. 27, pp. 207-216, 1996.

[48] Y. Epstein, J. Rosenblum, R. Burstein, and M. Sawka, "External load can alter the enery cost of prolonged exercise," Eur J Appl Physiol, vol. 57, pp. 243-247, 1988. 
[49] J. Patton, J. Kaszuba, R. Mell, and K. Reynolds, "Physiological responses to prolonged treadmill walking with external loads," Eur J Appl Physiol, vol. 63, pp. 89-93, 1991.

[50] V. Torvik, E. Triantaphyllow, T. Liao, and S. Waly, "Predicting muscle fatigue via electromyography: A comparative study," in Proceedings of the 25th International Conference on Computers and Industrial Engineering, pages 27r-280, 1999.

[51] G. Brooks, T. Fahey, and T. White, Exercise physiology: human bioenergetics and its applications (2nd ed.). Mayfield Publishing Co. Mountain View, CA, 1996.

[52] M. Febbraio and J. Dancey, "Skeletal muscle energy metabolism during prolonged fatiguing exercise," J Appl Physiol, vol. 87, pp. 2341-2347, 1999.

[53] T. W. Beck, T. J. Housh, G. O. Johnson, J. P. Weir, J. T. Cramer, J. W. Coburn, and M. H. Malek, "Comparison of fourier and wavelet transform procedures for examining the mechanomyographic and electromyographic frequency domain responses during fatiguing isokinetic muscle actions of the biceps brachii," Journal of Electromyography and Kinesiology, vol. 15, no. 2, pp. 190-199, 2005.

[54] E. A. Clancy, D. Farina, and R. Merletti, "Cross-comparison of time- and frequency-domain methods for monitoring the myoelectric signal during a cyclic, force-varying, fatiguing hand-grip task," Journal of Electromyography and Kinesiology, vol. 15, no. 3, pp. 256-265, 2005.

[55] D. MacIsaac, P. Parker, and R. Scott, "The short-time fourier transform and muscle fatigue assessment in dynamic contractions," Journal of Electromyography and Kinesiology, vol. 11, no. 6, pp. 439-449, 2001.

[56] H. Oka, "Estimation of muscle fatigue by using emg and muscle stiffness," in 18th Annual International Conference of the IEEE Engineering in Medicine and Biology Society, Amsterdam 1996, 1996.

[57] C. DeLuca, "The use of surface electromyography in biomechanics," Journal of Applied Biomechanics, vol. 13, pp. 135-163, 1997.

[58] L. P. J. Selen, P. J. Beek, and J. H. van Dien, "Fatigue-induced changes of impedence and performance in target tracking," Experimental Brain Research, vol. 181, pp. 99-108, 2007.

[59] D. Farina, L. Fattorini, F. Felici, and G. Filligoi, "Nonlinear surface emg analysis to detect changes of motor unit conduction velocity and synchronization," Journal of Applied Physiology, vol. 93, pp. 1753-1763, 2002. 
[60] M. Arihara and K. Sakamoto, "Contribution of motor unit activity enhanced by acute fatigue to physiological tremor," Electromyogr Clin Neurophysiol, vol. 39, pp. 235-247, 1999.

[61] S. K. Hunter, A. Critchlow, I.-S. Shin, and R. M. Enoka, "Fatigability of the elbow flexor muscles for a sustained submaximal contraction is similar in men and women matched for strength," Journal of Applied Physiology, vol. 96, pp. 195-202, 2004.

[62] L. Skjaervan, S. Hollup, and N. Reuter, "Normal mode analysis for proteins," Journal of Molecular Structure: THEOCHEM, vol. 898, pp. 42-48, 2009.

[63] A. K. Mazur, "Hierarchy of fast motions in protein dynamics," Journal of Physical Chemistry B, vol. 102, pp. 473-479, 1998.

[64] A. K. Mazur, "Quasi-hamiltonian equations of motion for internal coordinate molecular dynamics of polymers," Journal of Computational Chemistry, vol. 18(11), pp. 1354-1364, 1997.

[65] W. T. F. Encyclopedia, "Energy landscapes," http://en.wikipedia.org/wiki/Energy-landscape, Tech. Rep., 2010, date Accessed: October 2010.

[66] A. Alexandros, M. Otten, P. Nguyen, R. Hegger, and G. Stock, "Construction of the free energy landscape of biomolecules via dihedral angle principal component analysis," Journal of Chemical Physics, vol. 128, p. 245102, 2008.

[67] P. Petrone and V. Pande, "Can conformational change be described by only a few normal modes?" Biophysical Journal of Medicinal Chemistry, vol. 90, pp. 1583-1593, 2006.

[68] A. Kolinski and J. Skolnick, "Reduced models of proteins and their applications," Polymer, vol. 45, pp. 511-524, 2004.

[69] Y. Mu, H. Nguyen, and G. Stock, "Energy landscape of a small peptide revealed by dihedral angle principle component analysis," Proteins: Structure, Function, and Bioinformatics, vol. 64, pp. 795-797, 2006.

[70] D. Tang, A. Li, P. Attar, and E. H. Dowell, "Reduced order dynamic model for polysaccharides molecule attached to an atomic force microscope," Journal of Computational Physics, vol. 201, pp. 723-752, 2004.

[71] M. Lu, B. Poon, and J. Ma, "A new method for course-grained elastic normal mode analysis," Journal of Chemical Theory and Computation, vol. 2, pp. 464-471, 2006. 
[72] F. Lange and H. Grubmuller, "Collective langevin dynamics of conformational motions in proteins," Journal of Chemical Physics, vol. 124, pp. 214903$214921,2006$.

[73] A. Altis, R. Nguyen, H.Pand Hegger, and G. Stock, "Dihedral angle principle component analysis of molecular dynamics simulations," Journal of Chemical Physics, vol. 126, pp. 24 411-24 421, 2007.

[74] T. Sauer, J. A. Yorke, and M. Casdagli, "Embedology," J. Stat. Phys., vol. 65, no. 3-4, pp. 579-616, 1991.

[75] M. Kennel, R. Brown, and H. Abarbanel, "Determining embedding dimension for phase-space reconstruction using a geometrical construction," Physical Reviews A, vol. 45 (6), pp. 3403-3411, 1992.

[76] A. Fraser and H. Swinney, "Independent coordinates for strange attractors from mutual information," Physical Reviews A, vol. 33 (2), pp. 1134-1140, 1986.

[77] H. Kantz and T. Schreiber, Nonlinear Time Series Analysis. Cambridge University Press, 2004.

[78] M. Casdagli, S. Eubank, J. Farmer, and J. Gibson, "State space reconstruction in the presence of noise," Physica D: Nonlinear Phenomena, vol. 51, no. 1-3, pp. 52 - 98, 1991. [Online]. Available: http://www.sciencedirect.com/ science/article/B6TVK-46MV07J-7/2/fe34efc4f128e33716d7003ee4dd4d45

[79] J. Friedman, J. Bentley, and R. Finkel, "An algorithm for finding best matches in logarithmic expected time," ACM Transactions on Mathematical Sofware, vol. 3, pp. 209-226, 1977.

[80] F. Moon and P. Holmes, "A magnetoelastic strange attractor," Journal of Sound and Vibration, vol. 65, pp. 275-296, 1979.

[81] D. Gates and J. Dingwell, "Effects of muscle fatigue on variability and temporal correlations in movement timing errors," Experimental Brain Research, vol. 187, pp. 573-585, 2008.

[82] D. A. Winter, Biomechanics and motor control of human movement. John Wiley \& Sons. Inc., 2005.

[83] A. Perotto, M and E. Delagi, F, Anatomical guide for the electromyographer: the limbs and trunk. Charles C Thomas, 2005.

[84] F. Veldpaus, H. Woltrin, and L. Dortmans, "A least-squares algorithm for the equiform transformation from spatial marker co-ordinates," Journal of Biomechanics, vol. 21, pp. 45-54, 1998. 
[85] R. Schmidt, C. Disselhorst-Klug, J. Silny, and G. Rau, "A marker-based measurement procedure for unconstrained wrist and elbow motions," J Biomech, vol. 32, pp. 615-621, 1999.

[86] G. Wu, F. van der Helm, M. Veeger, HE amd Makhsous, P. Van Roy, C. Anglin, J. Nagels, A. Karduna, K. McQuade, X. Wang, F. Werner, and B. B, "Isb recommendation on definitions of joint coordinate systems of various joints for the reporting of human joint motion-part ii: shoulder, elbow, wrist and hand," Journal of Biomechanics, vol. 38, pp. 981-992, 2005.

[87] B. Hingtgen, J. McGuire, M. Wang, and G. Harris, "An upper extremity kinematic model for evaluation of hemiparetic stroke," Journal of Biomechanics, vol. 39, pp. 681-688, 2006.

[88] S. Rao, E. Bontruger, J. Gronley, C. Newsam, and J. Perry, "Threedimensional kinematics of wheelchair propulsion," IEEE Transactions on Rehabilitation Engineering, vol. 4, no. 3, pp. 152-160, 1996.

[89] D. H. Gates, "The role of muscle fatigue on movement timing and stability during repetitive tasks," Ph.D. dissertation, University of Texas at Austin, 2009 .

[90] N. Leao, R and A. Burne, J, "Continuous wavelet transform in the evaluation of stretch reflex responses from surface emg," Journal of Neuroscience Methods, vol. 133, pp. 115-125, 2004.

[91] W. L. Jorgensen and J. Tirado-Rives, "The opls potential functions for proteins. energy minimizations for crystals of cyclic peptides and crambin," Journal of the American Chemical Society, vol. 110(6), pp. 1657-1666, 1998.

[92] W. L. Jorgensen, D. S. Maxwell, and J. Tirado-Rives, "Development and testing of opls all atom force field on conformational energetics and properties of organic liquids," Journal of the American Chemical Society, vol. 118, pp. $11225-11236,1996$.

[93] M. Heller, M. Sukopp, N. Tsomaia, M. John, B. Mierke, D.Fand Reif, and H. Kessler, "The conformation of cyclo(-d-pro-ala4-) as a model for cyclic pentapeptides of the dl4 type," Journal of the American Chemical Society, vol. 128, pp. $13806-13814,2006$.

[94] W. Humphrey, A. . Dalke, and K. Schulten, "Vmd - visual molecular dynamics," Journal of Molecular Graphics, vol. 14, pp. 33-38, 1996.

[95] H. Sadeghi, P. Allard, F. Prince, and H. Labelle, "Symmetry and limb dominance in able-bodied gait: a review," Gait and Posture, vol. 12, pp. 34-45, 2000. 
APPENDIX A

List of Acronyms 
Table A.1. List of acronyms used throughout this dissertation

FNN False Nearest Neighbor
AMI Average Mutual Information
PSW Phase Space Warping
STRMP Short-time Reference Model Prediction
SOD Smooth Orthogonal Decomposition
SOM Smooth Orthogonal Mode
SPM Smooth Projection Mode
SOC Smooth Orthogonal Coordinate
NSOC Nonlinear Smooth Orthogonal Coordinate
POD Proper Orthogonal Decomposition
POM Proper Orthogonal Mode
PC Proper Coordinates
LNM Linear Normal Mode
PCA Principle Component Analysis
dPCA Dihedral Principle Component Analysis
NMR Nuclear Magnetic Resonance
EMG Electromyography
MNF Mean Power Spectral Frequency
IMNF Instantaneous Mean Frequency
$\dot{\mathrm{V}} \mathrm{O}_{2} \quad$ Breath by Breath Oxygen Consumption
OPLS Optimized Potentials for Liquid Simulation
DMSO Dimethyl Sulfoxide
RMSD Root Mean Square Deviation 


\section{APPENDIX B}

Sawing and Walking Subjects 


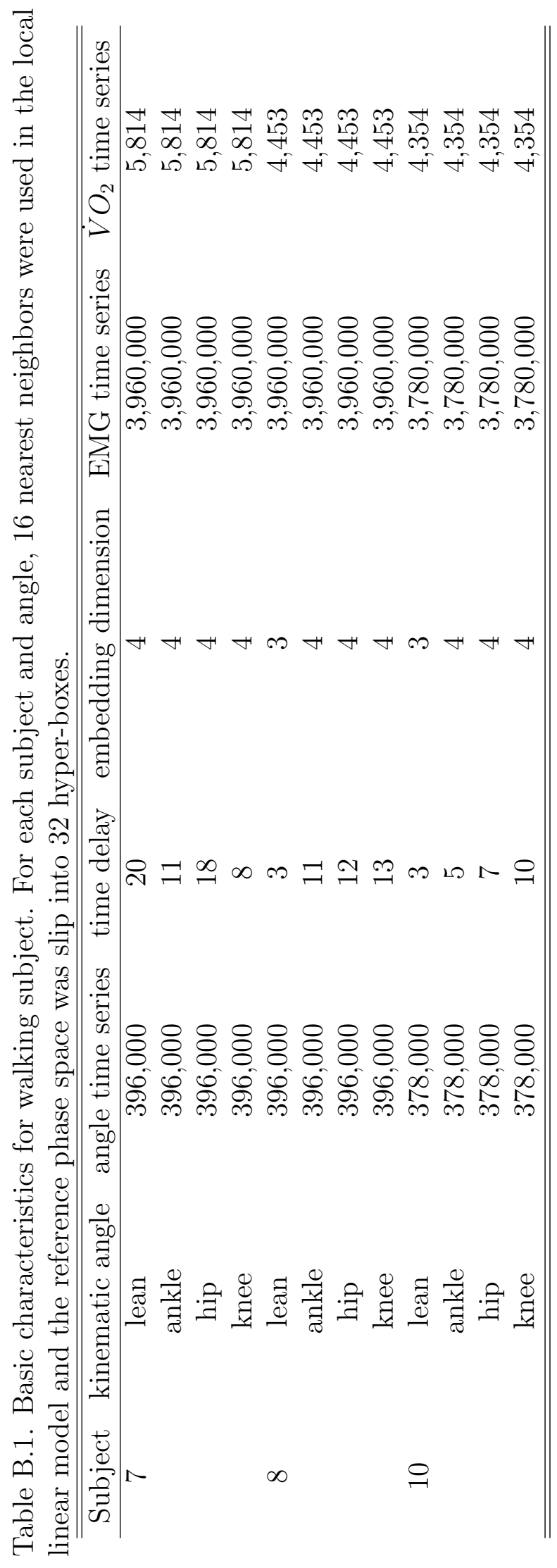




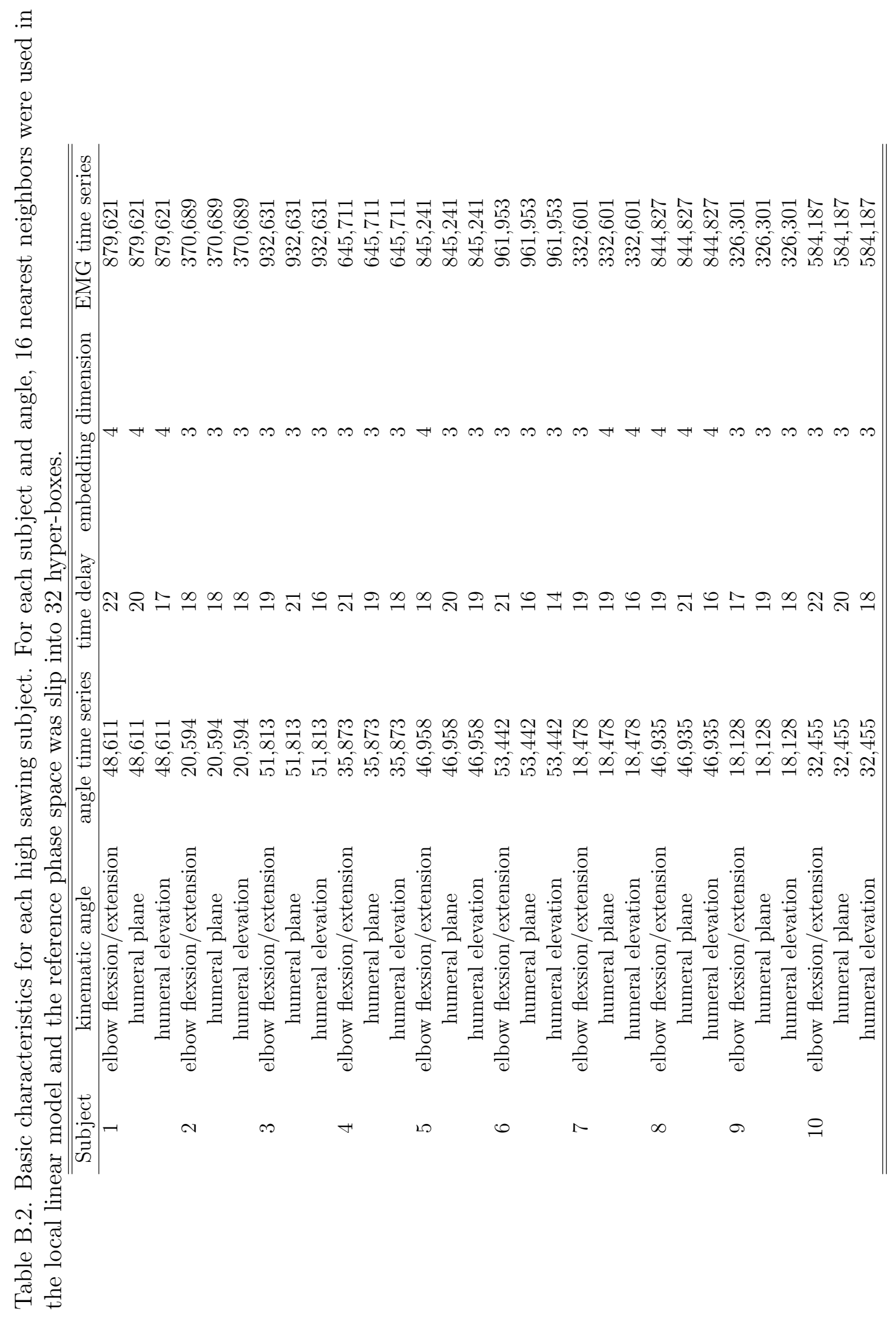




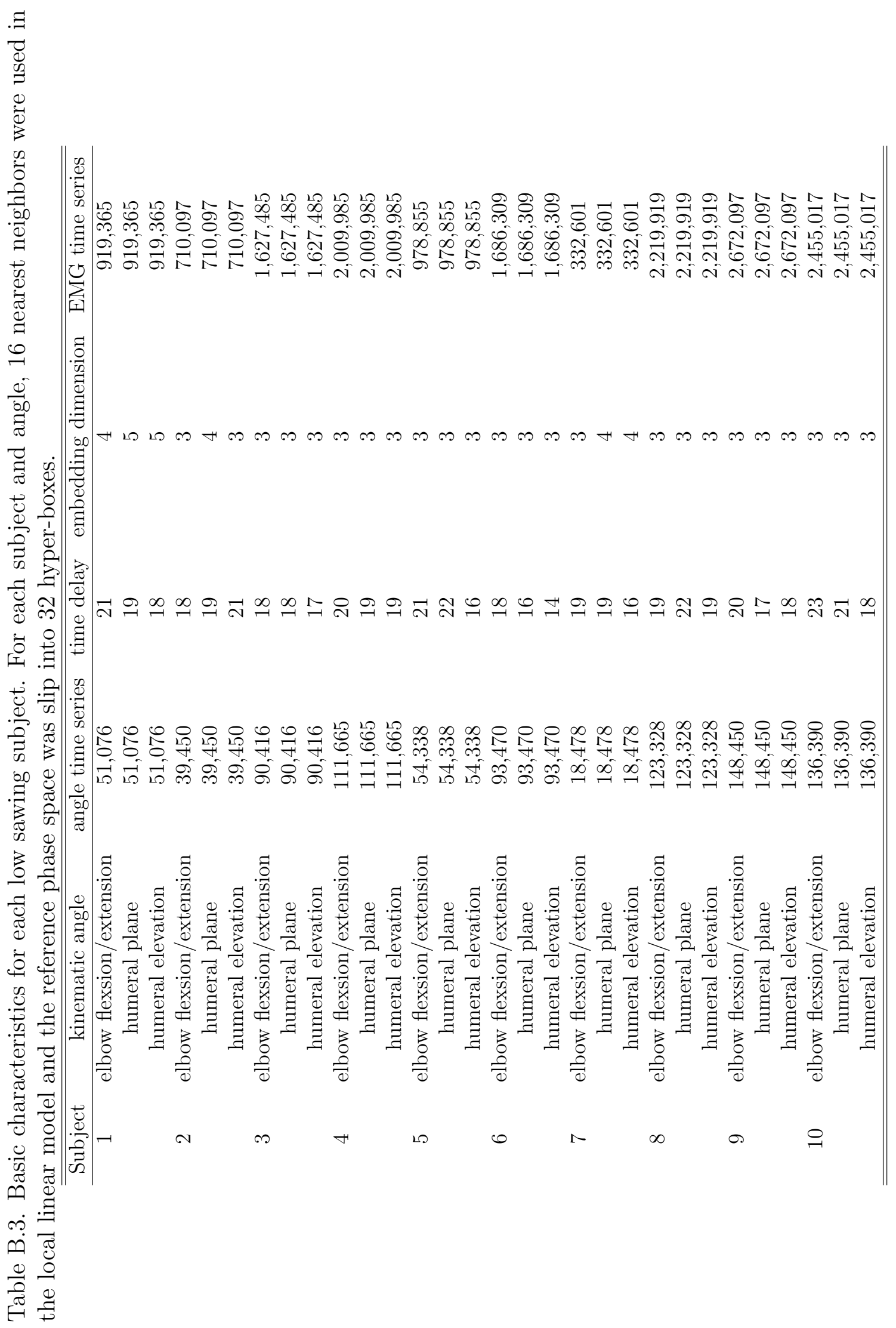




\section{APPENDIX C}

SOCs Projections onto EMG Trends: Sawing Subjects 

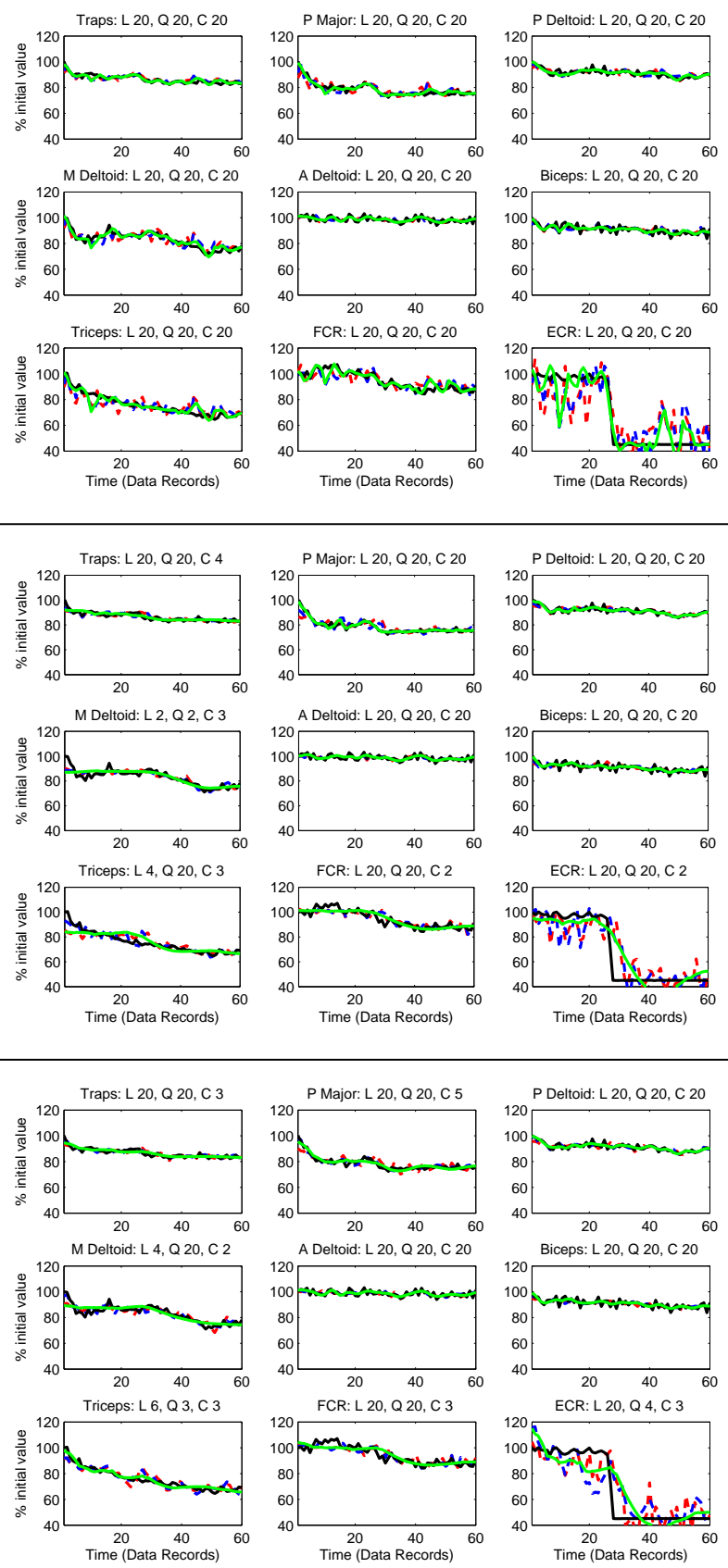

Figure C.1. Linear combinations of linear (red --), quadratic (blue --), and cubic (green -) SOCs projections onto MNF (black - ) markers in a least squares sense for subject 1 high sawing experiment: elbow flexion/extension (top), humeral plane (middle), and humeral elevation (bottom) angles. 

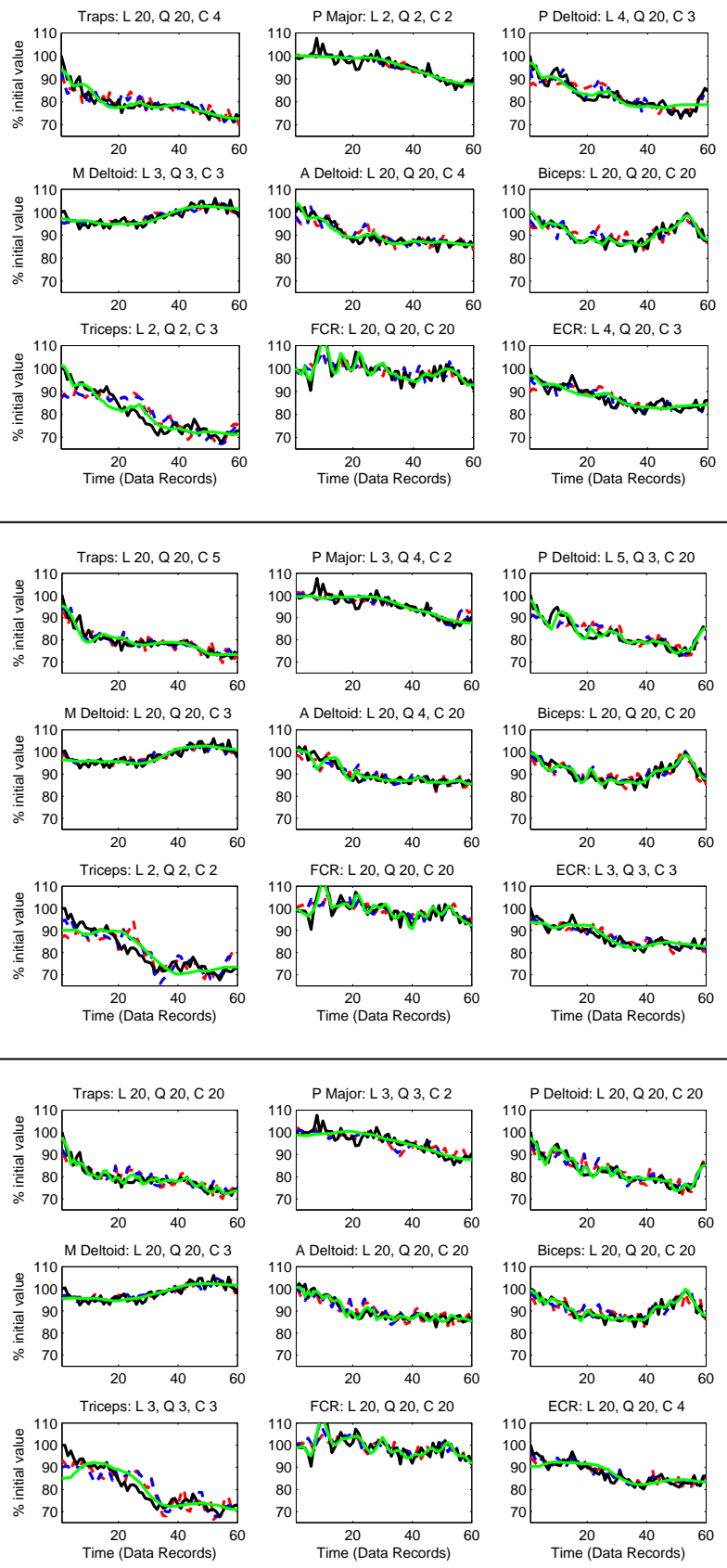

Figure C.2. Linear combinations of linear (red --), quadratic (blue --), and cubic (green - ) SOCs projections onto MNF (black - ) markers in a least squares sense for subject 1 low sawing experiment: elbow flexion/extension (top), humeral plane (middle), and humeral elevation (bottom) angles. 

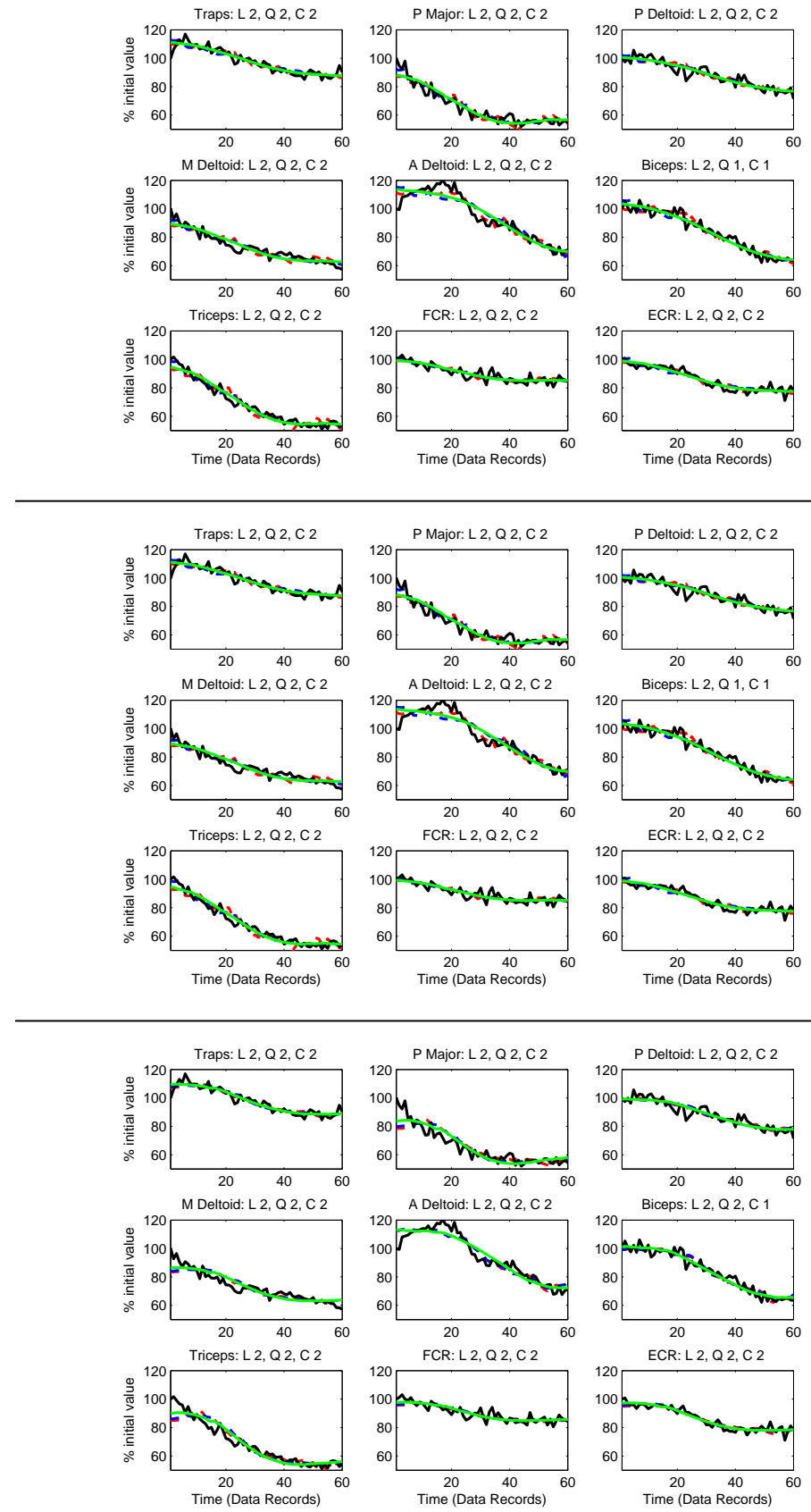

Figure C.3. Linear combinations of linear (red --), quadratic (blue --), and cubic (green -) SOCs projections onto MNF (black - ) markers in a least squares sense for subject 2 high sawing experiment: elbow flexion/extension (top), humeral plane (middle), and humeral elevation (bottom) angles. 

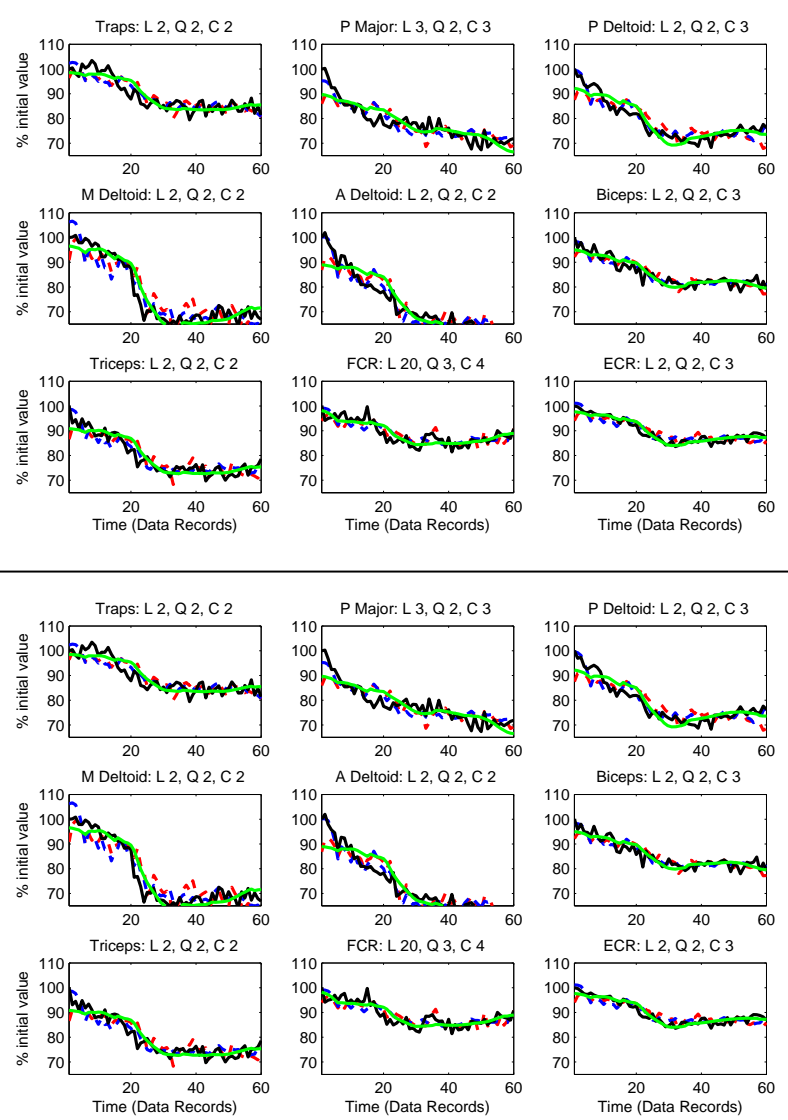

Figure C.4. Linear combinations of linear (red --), quadratic (blue --), and cubic (green -) SOCs projections onto MNF (black -) markers in a least squares sense for subject 2 low sawing experiment: elbow flexion/extension (top), humeral plane (bottom) angles. 

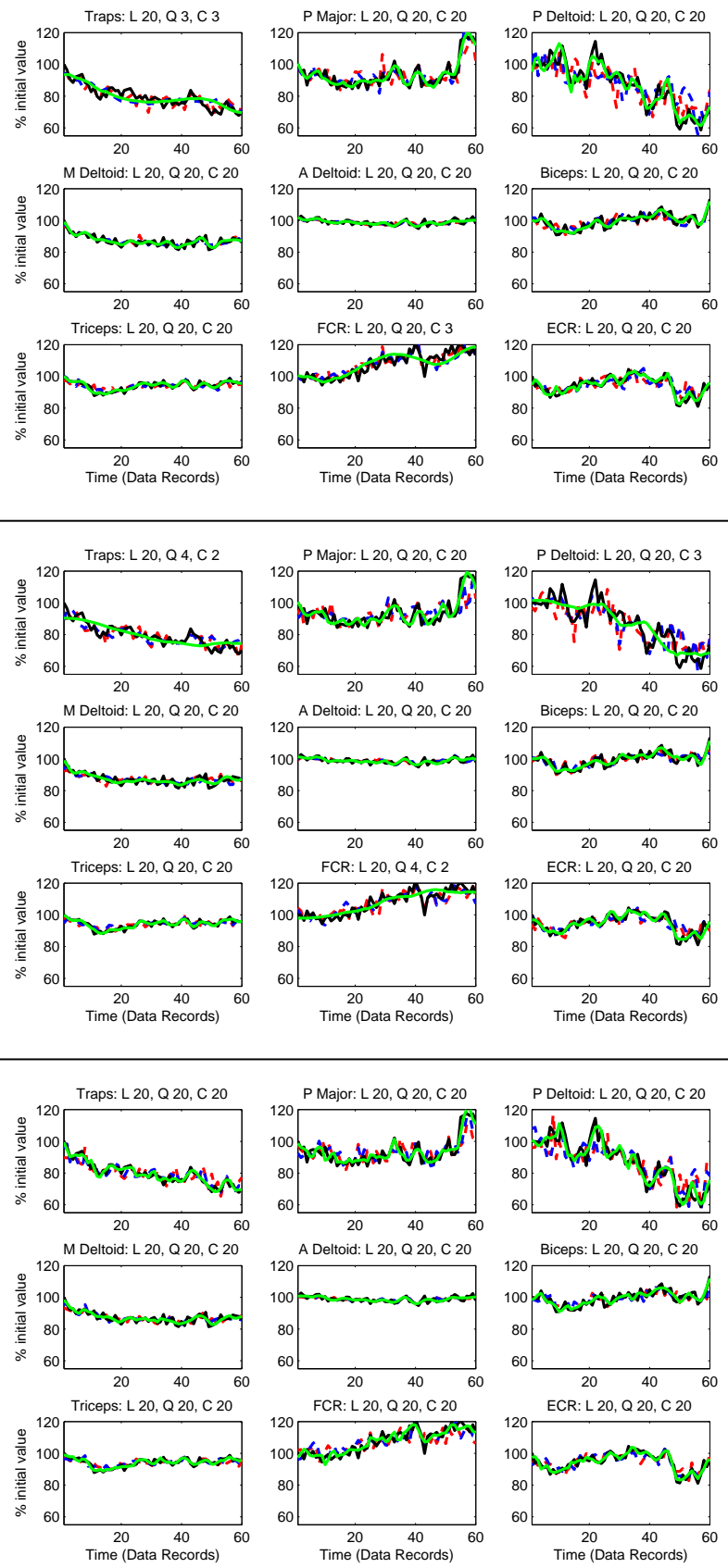

Figure C.5. Linear combinations of linear (red --), quadratic (blue --), and cubic (green -) SOCs projections onto MNF (black - ) markers in a least squares sense for subject 3 high sawing experiment: elbow flexion/extension (top), humeral plane (middle), and humeral elevation (bottom) angles. 

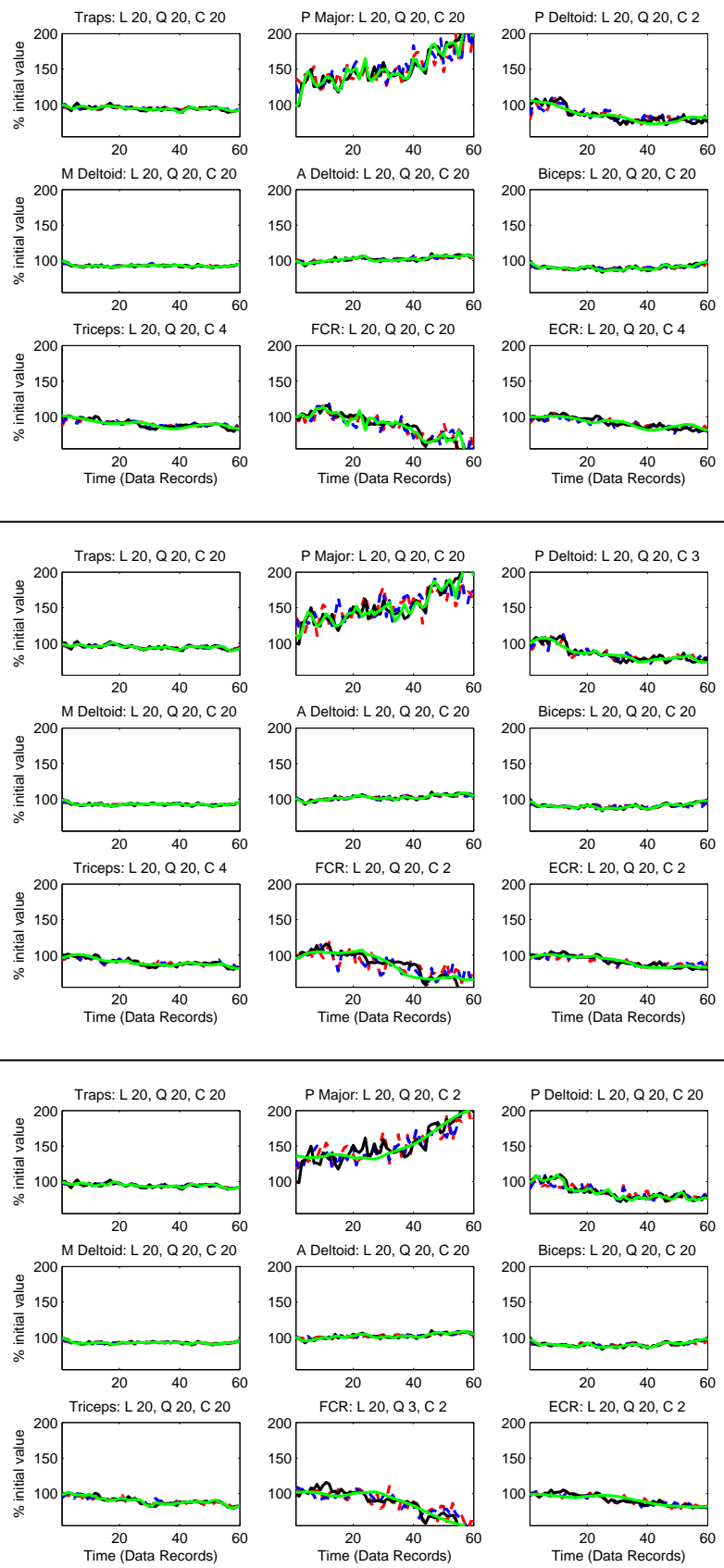

Figure C.6. Linear combinations of linear (red --), quadratic (blue --), and cubic (green -) SOCs projections onto MNF (black - ) markers in a least squares sense for subject 3 low sawing experiment: elbow flexion/extension (top), humeral plane (middle), and humeral elevation (bottom) angles. 

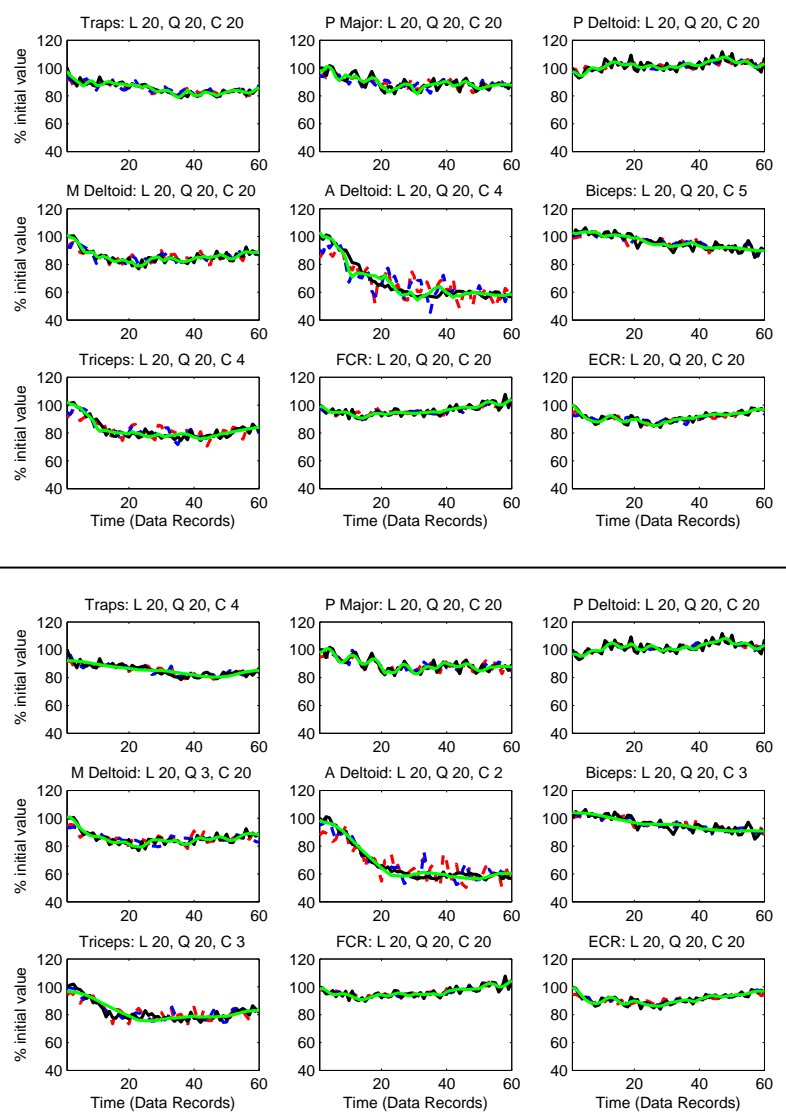

Figure C.7. Linear combinations of linear (red --), quadratic (blue --), and cubic (green -) SOCs projections onto MNF (black -) markers in a least squares sense for subject 4 high sawing experiment: elbow flexion/extension (top), humeral plane (bottom) angles. 

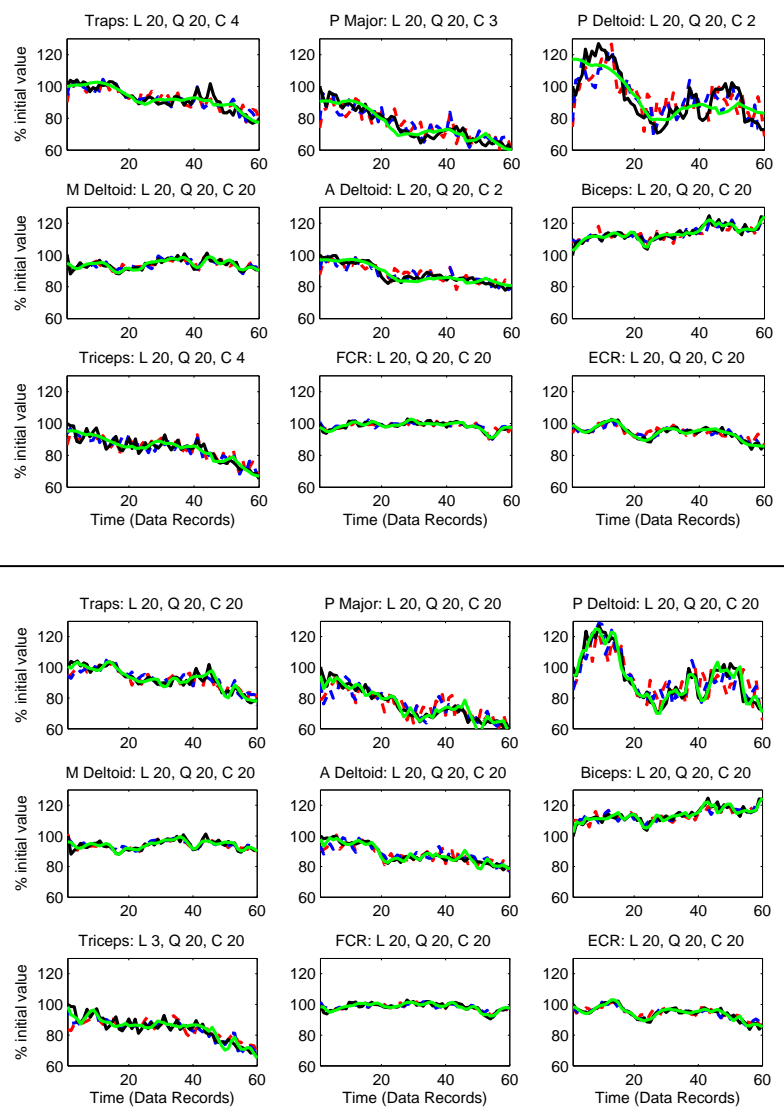

Figure C.8. Linear combinations of linear (red --), quadratic (blue --), and cubic (green -) SOCs projections onto MNF (black -) markers in a least squares sense for subject 4 low sawing experiment: elbow flexion/extension (top), humeral plane (bottom) angles. 

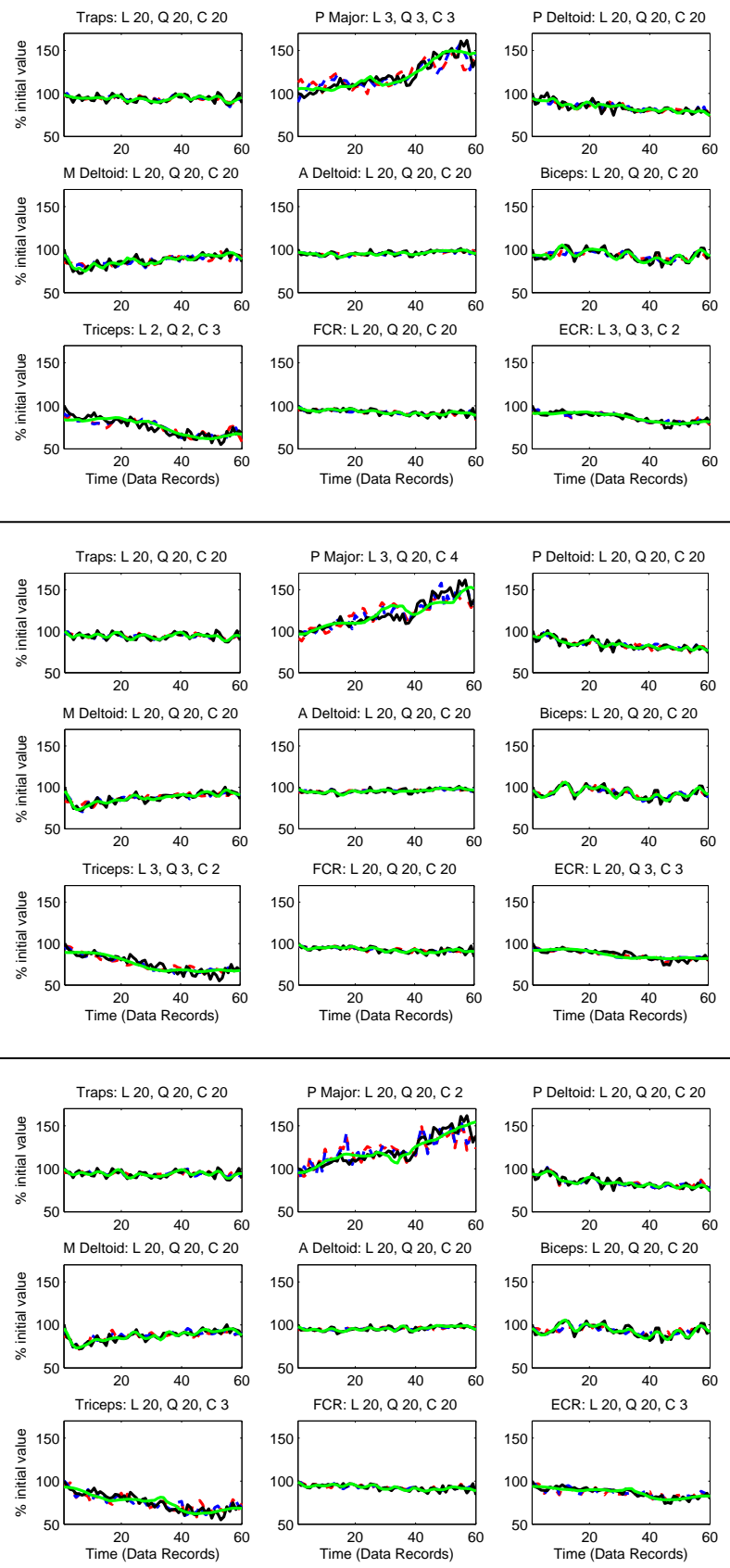

Figure C.9. Linear combinations of linear (red --), quadratic (blue --), and cubic (green -) SOCs projections onto MNF (black -) markers in a least squares sense for subject 5 high sawing experiment: elbow flexion/extension (top), humeral plane (middle), and humeral elevation (bottom) angles. 

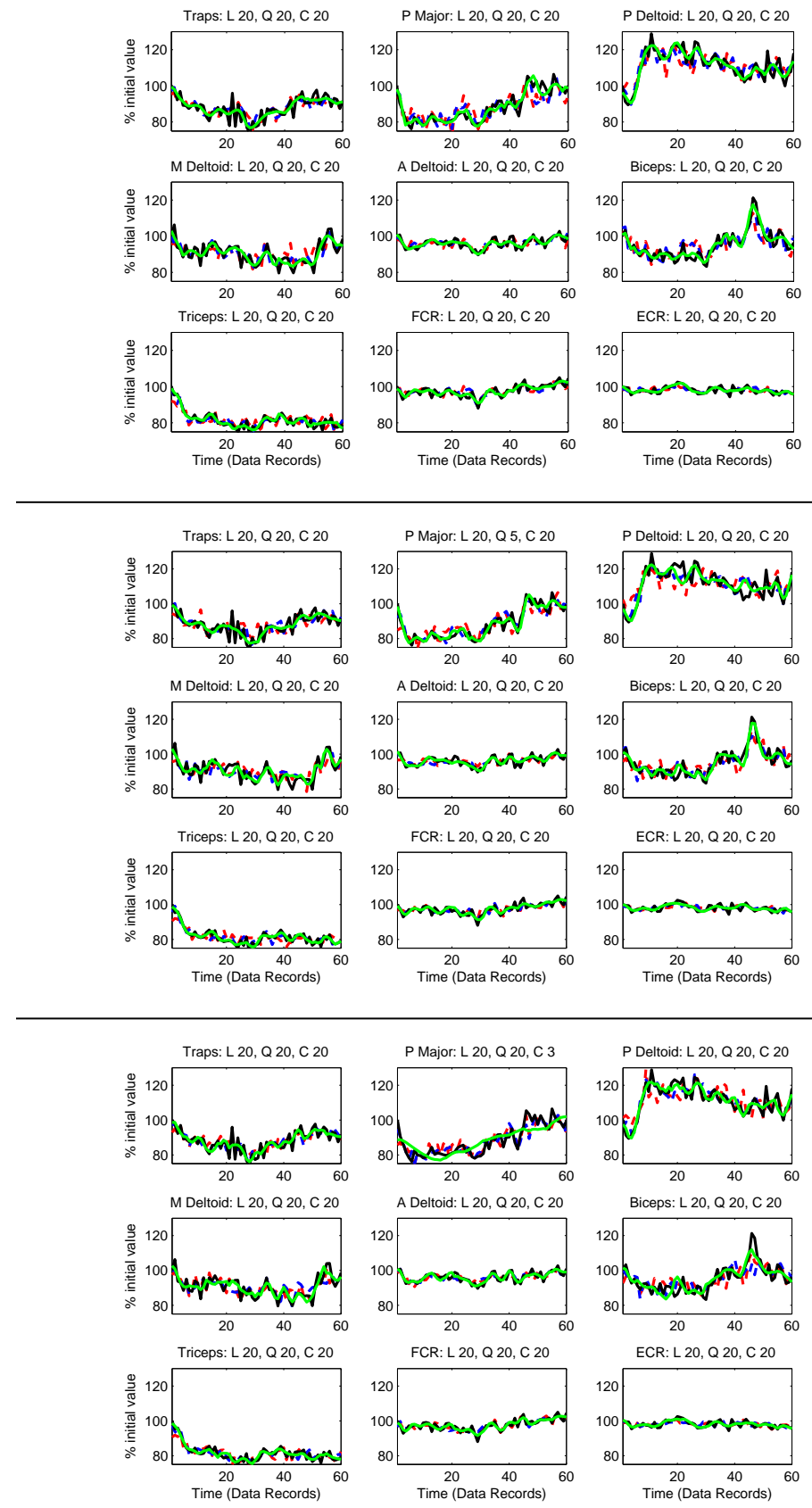

Figure C.10. Linear combinations of linear (red --), quadratic (blue --), and cubic (green - ) SOCs projections onto MNF (black - ) markers in a least squares sense for subject 5 low sawing experiment: elbow flexion/extension (top), humeral plane (middle), and humeral elevation (bottom) angles. 

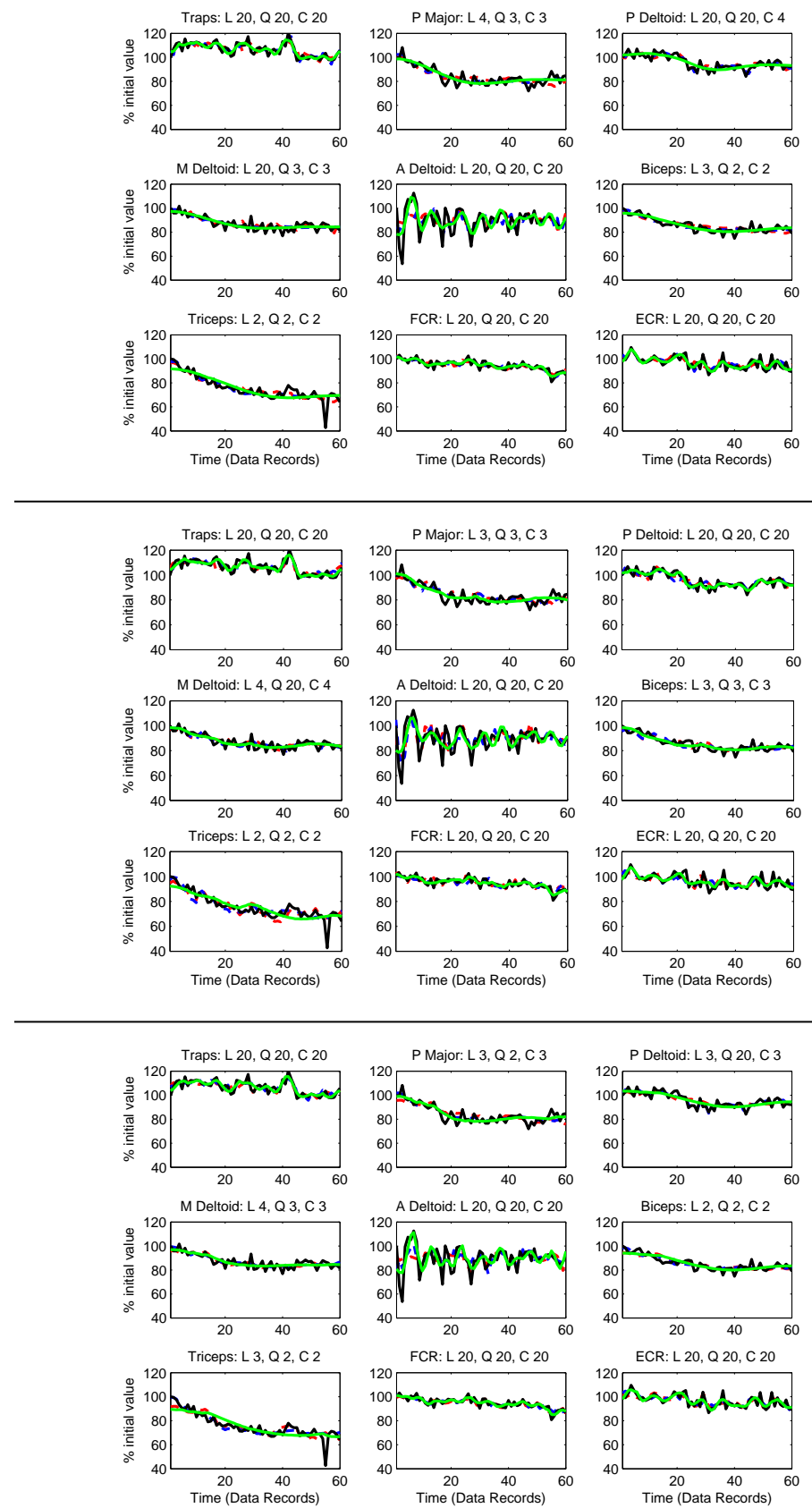

Figure C.11. Linear combinations of linear (red --), quadratic (blue --), and cubic (green - ) SOCs projections onto MNF (black - ) markers in a least squares sense for subject 6 high sawing experiment: elbow flexion/extension (top), humeral plane (middle), and humeral elevation (bottom) angles. 

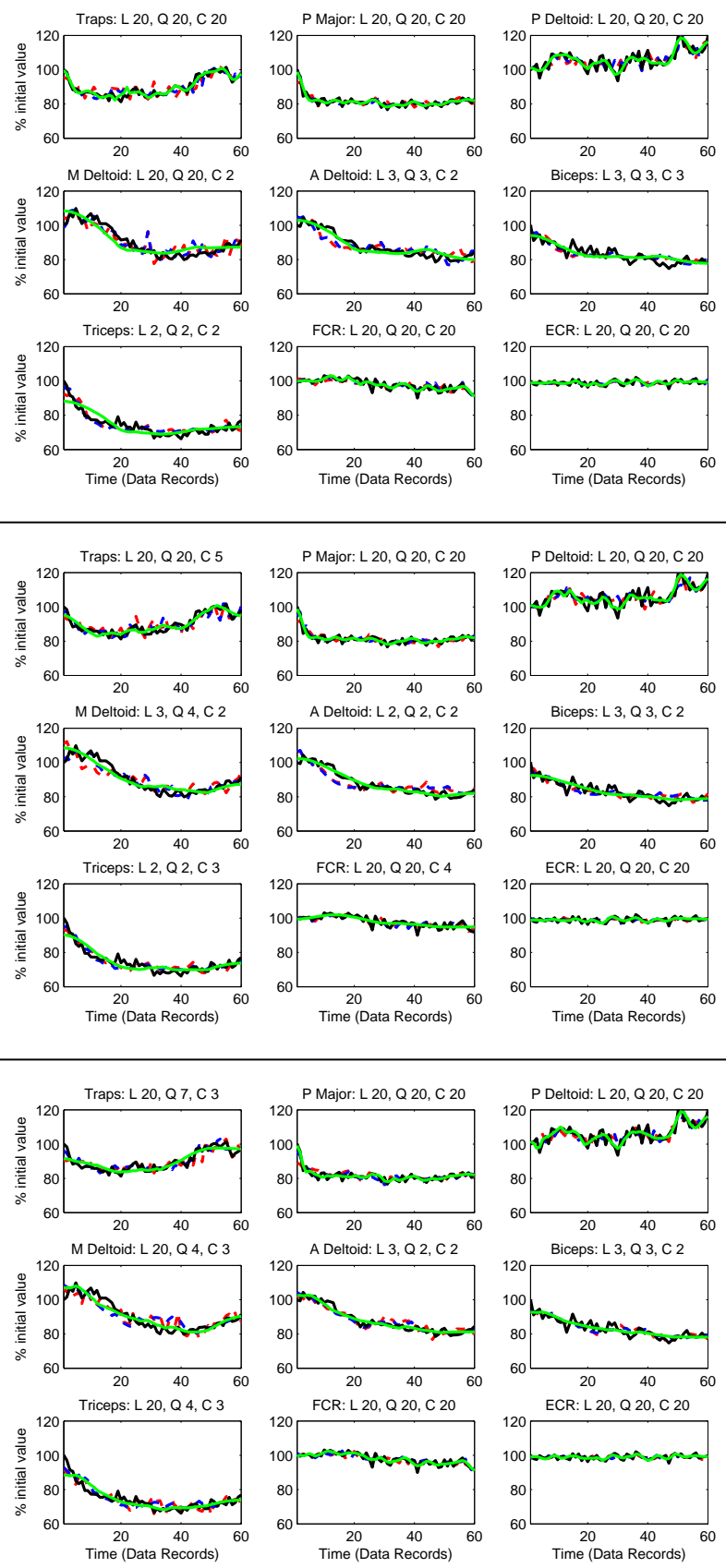

Figure C.12. Linear combinations of linear (red --), quadratic (blue --), and cubic (green -) SOCs projections onto MNF (black - ) markers in a least squares sense for subject 7 high sawing experiment: elbow flexion/extension (top), humeral plane (middle), and humeral elevation (bottom) angles. 

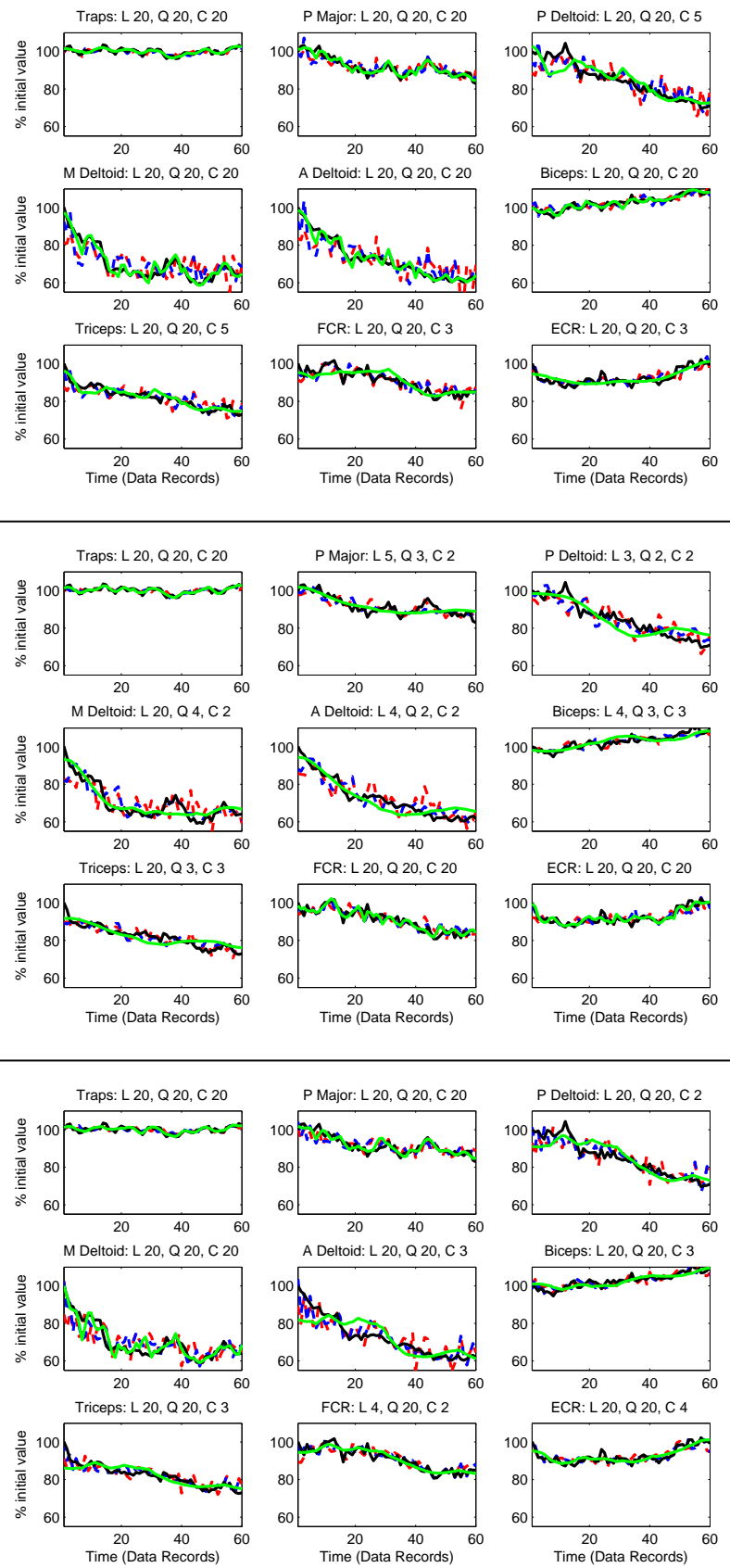

Figure C.13. Linear combinations of linear (red --), quadratic (blue --), and cubic (green - ) SOCs projections onto MNF (black - ) markers in a least squares sense for subject 7 low sawing experiment: elbow flexion/extension (top), humeral plane (middle), and humeral elevation (bottom) angles. 

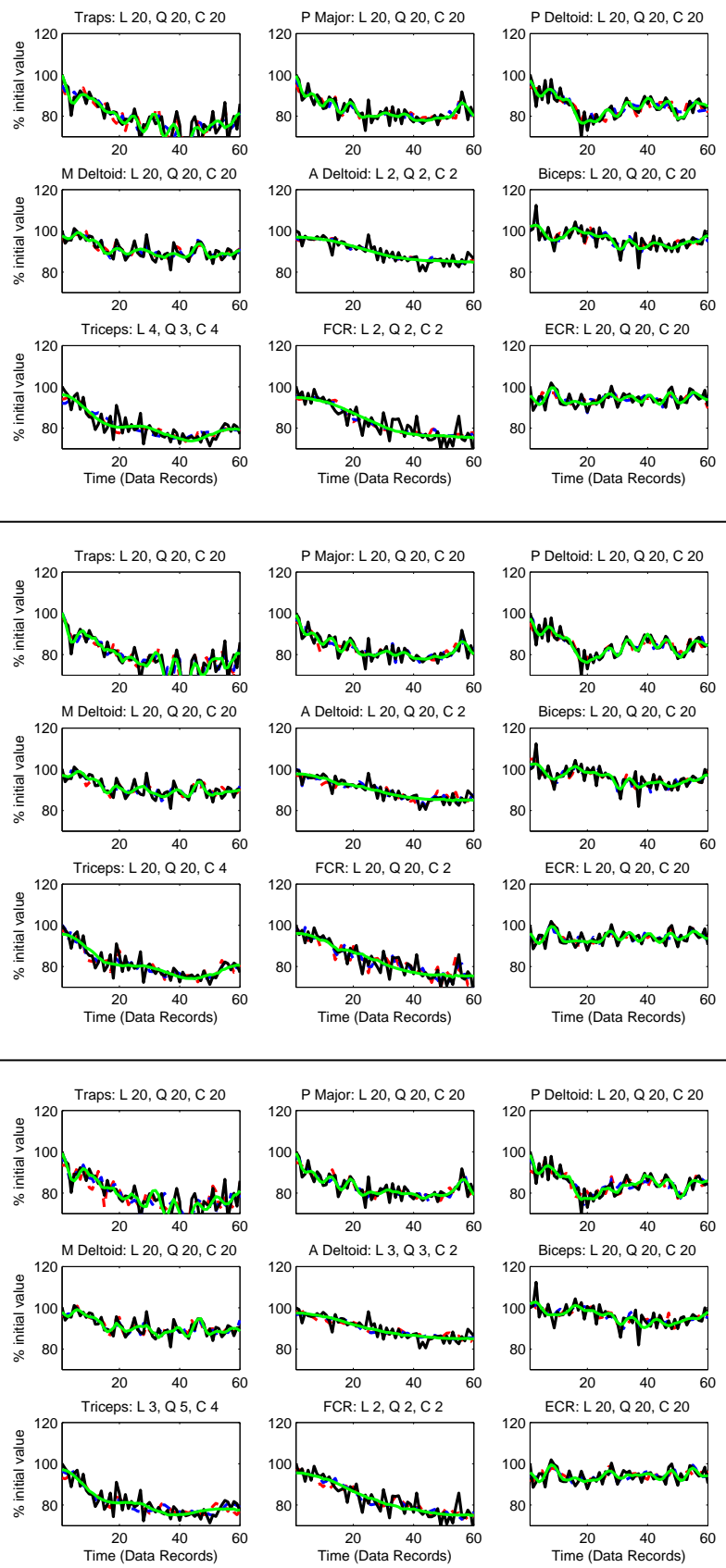

Figure C.14. Linear combinations of linear (red --), quadratic (blue --), and cubic (green -) SOCs projections onto MNF (black - ) markers in a least squares sense for subject 8 high sawing experiment: elbow flexion/extension (top), humeral plane (middle), and humeral elevation (bottom) angles. 

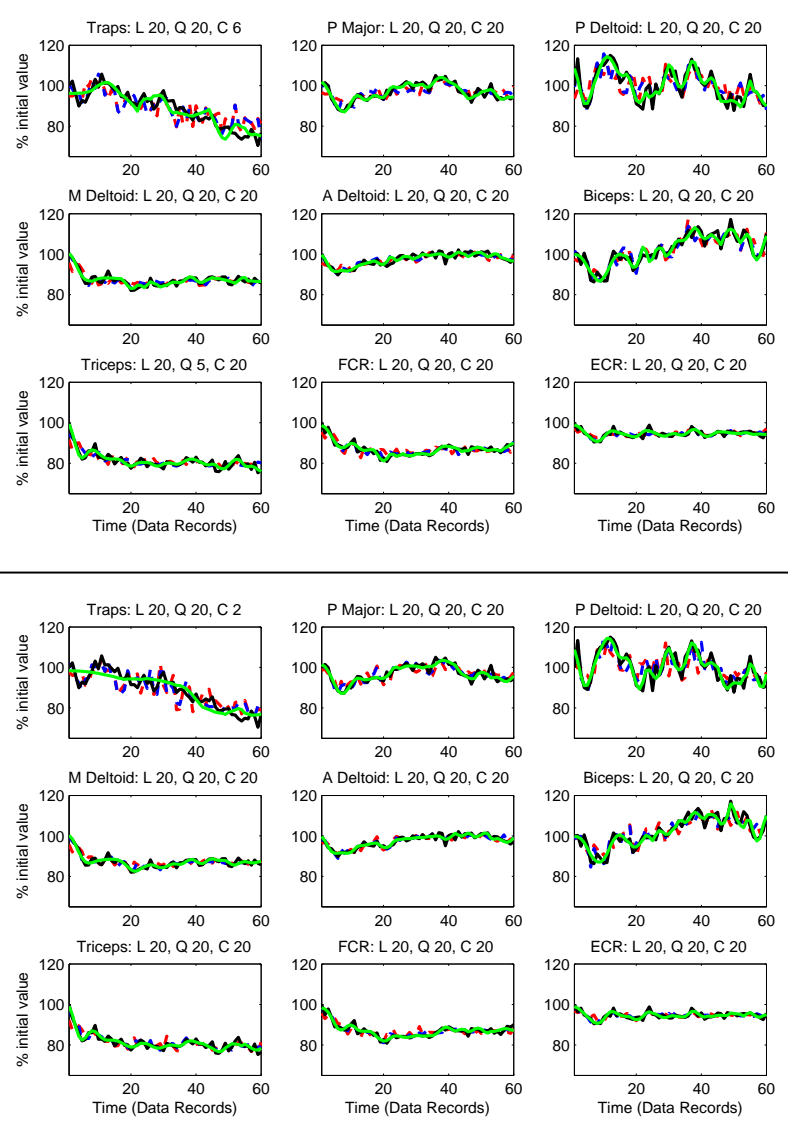

Figure C.15. Linear combinations of linear (red --), quadratic (blue --), and cubic (green -) SOCs projections onto MNF (black -) markers in a least squares sense for subject 8 low sawing experiment: elbow flexion/extension (top) and humeral elevation (bottom) angles. 

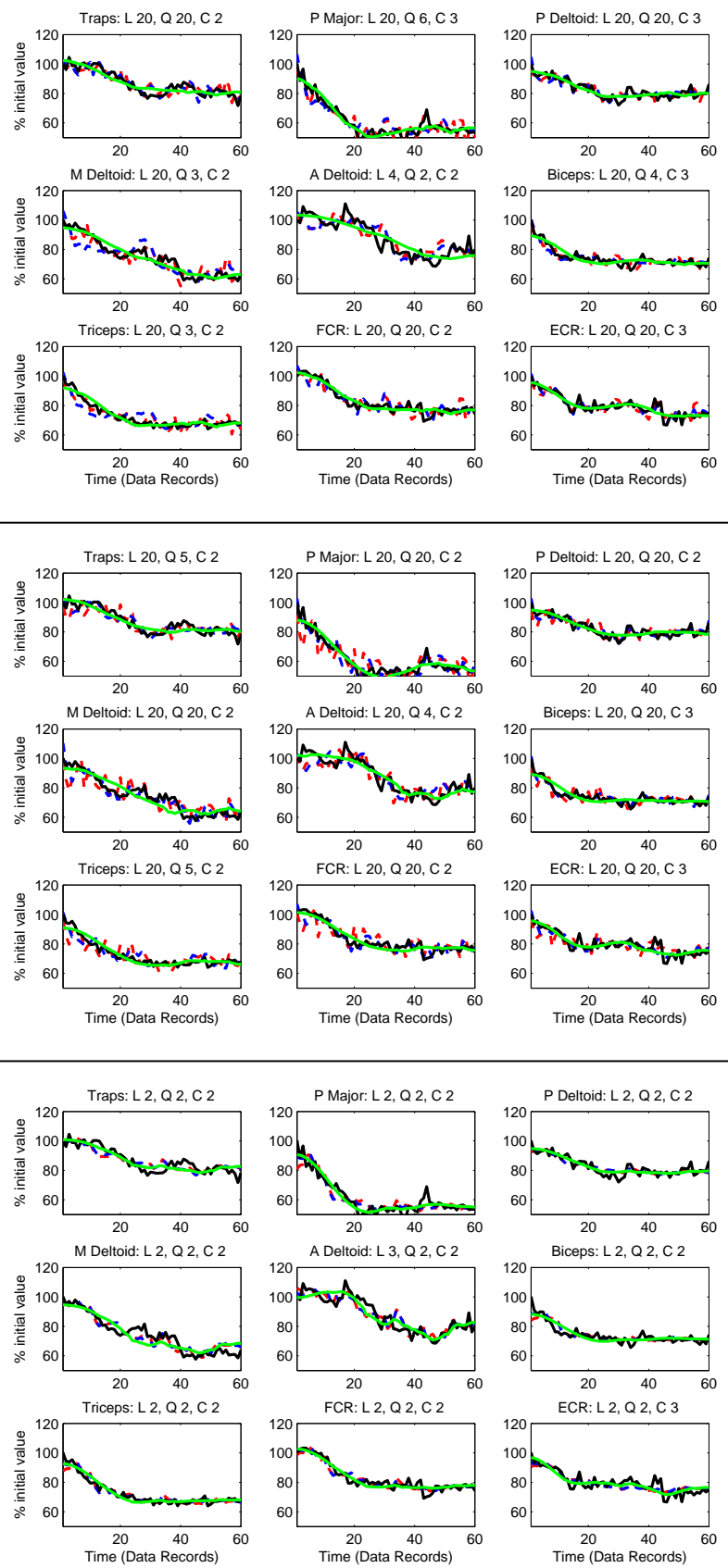

Figure C.16. Linear combinations of linear (red --), quadratic (blue --), and cubic (green -) SOCs projections onto MNF (black - ) markers in a least squares sense for subject 9 high sawing experiment: elbow flexion/extension (top), humeral plane (middle), and humeral elevation (bottom) angles. 

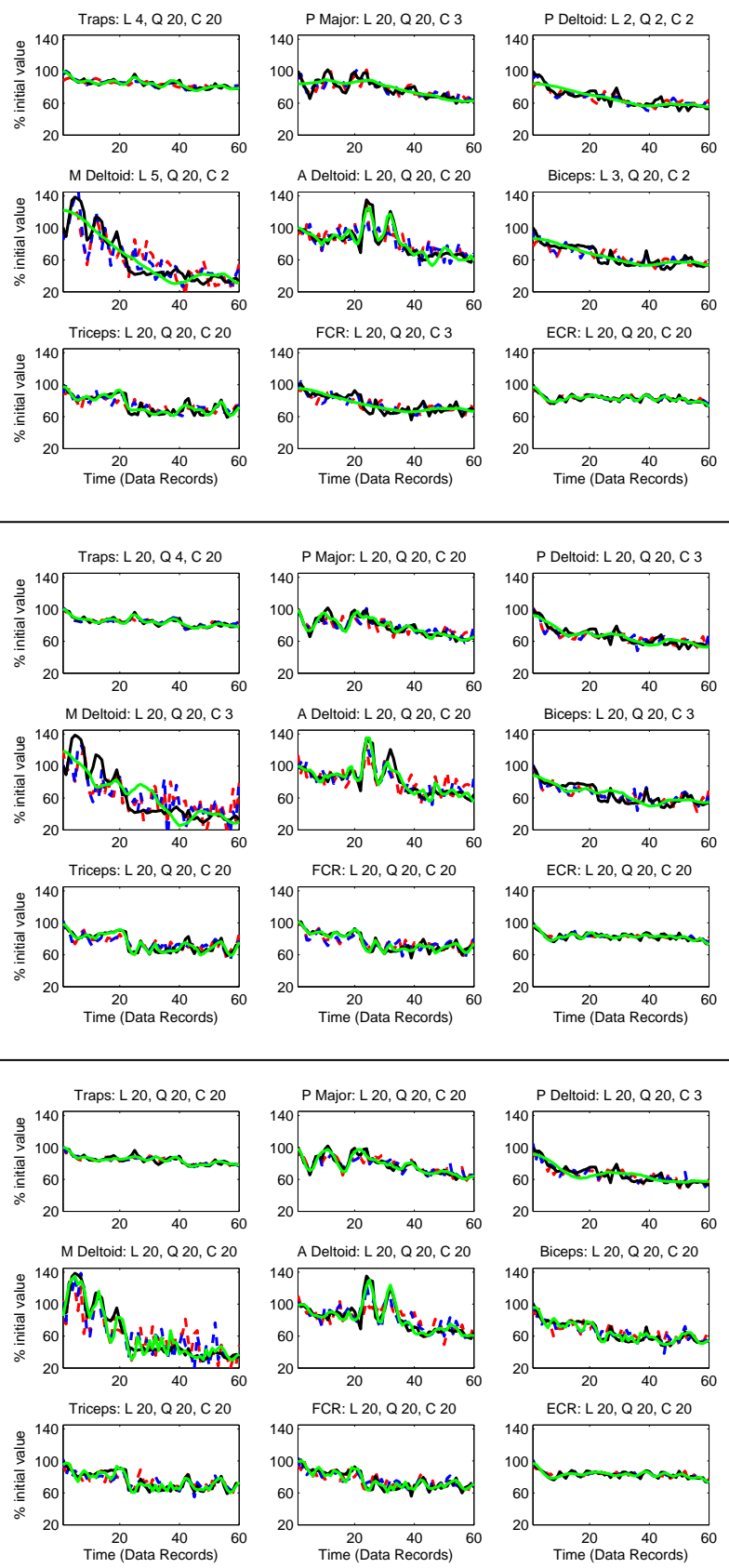

Figure C.17. Linear combinations of linear (red --), quadratic (blue --), and cubic (green - ) SOCs projections onto MNF (black - ) markers in a least squares sense for subject 9 low sawing experiment: elbow flexion/extension (top), humeral plane (middle), and humeral elevation (bottom) angles. 


\section{APPENDIX D}

SOCs Projections onto EMG Trends: Army Subjects 

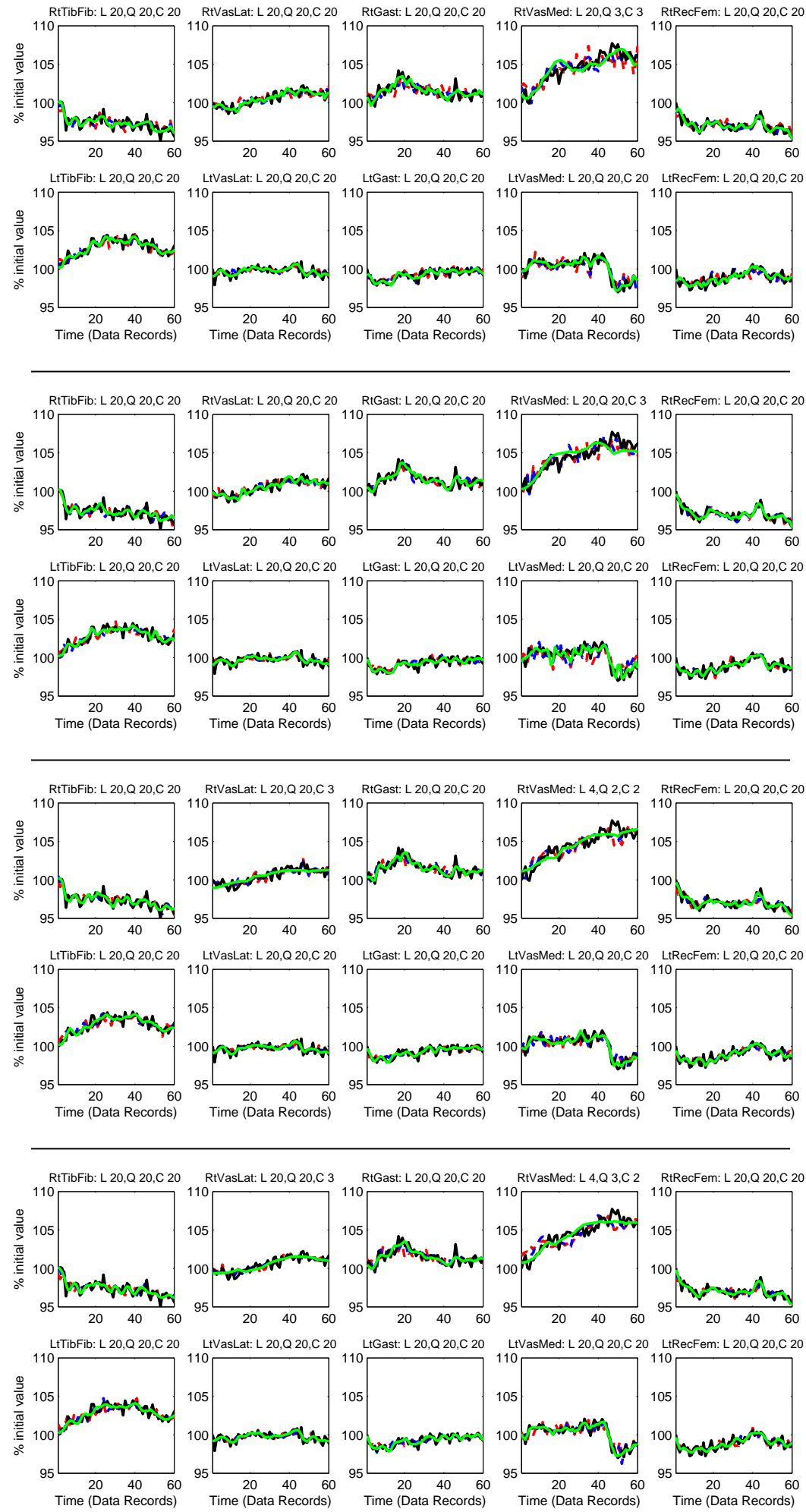

Figure D.1. Linear combinations of linear (red --), quadratic (blue --), and cubic (green -) SOCs projections onto MNF (black - ) markers in a least squares sense for for subject 7: lean (top), hip (middle top), ankle (middle bottom), and knee (bottom) angles in the army walking experiment. 

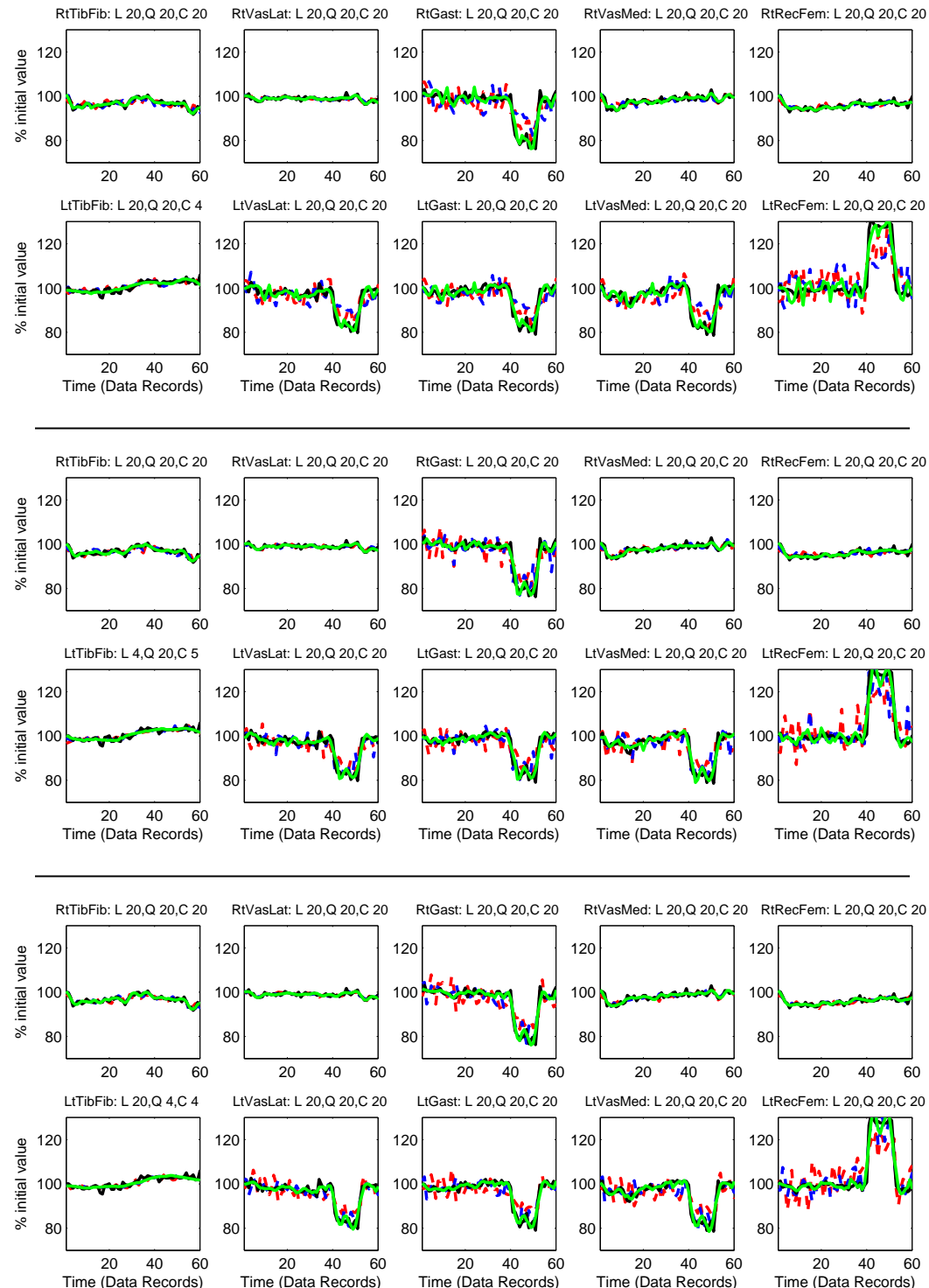

LtVasLat: $L 20, Q 20, C 20$

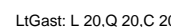

LtVasMed: L 20,Q 20,C 20 LtRecFem: L 20,Q 20,C 20
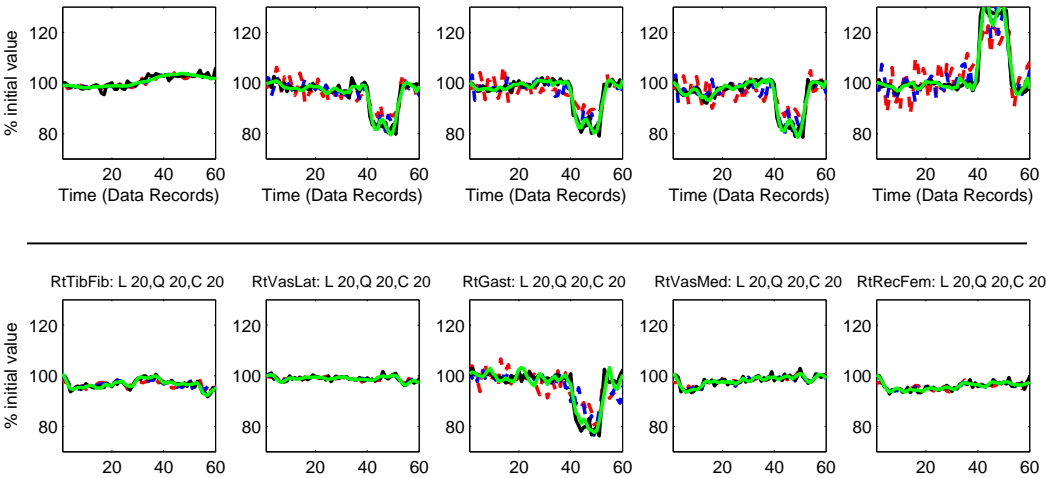

RtRecFem: $L$ 20,Q 20, C 20
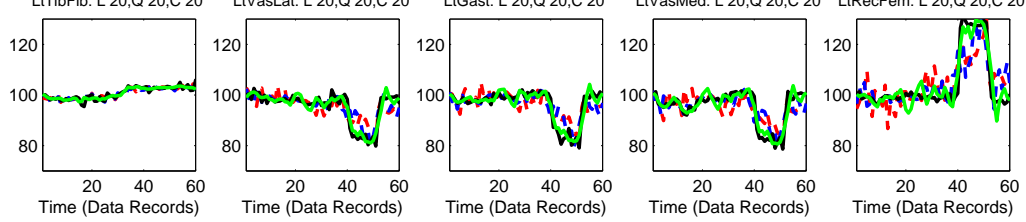

Figure D.2. Linear combinations of linear (red --), quadratic (blue --), and cubic (green -) SOCs projections onto MNF (black -) markers in a least squares sense for for subject 8: lean (top), hip (middle top), ankle (middle bottom), and knee (bottom) angles in the army walking experiment. 

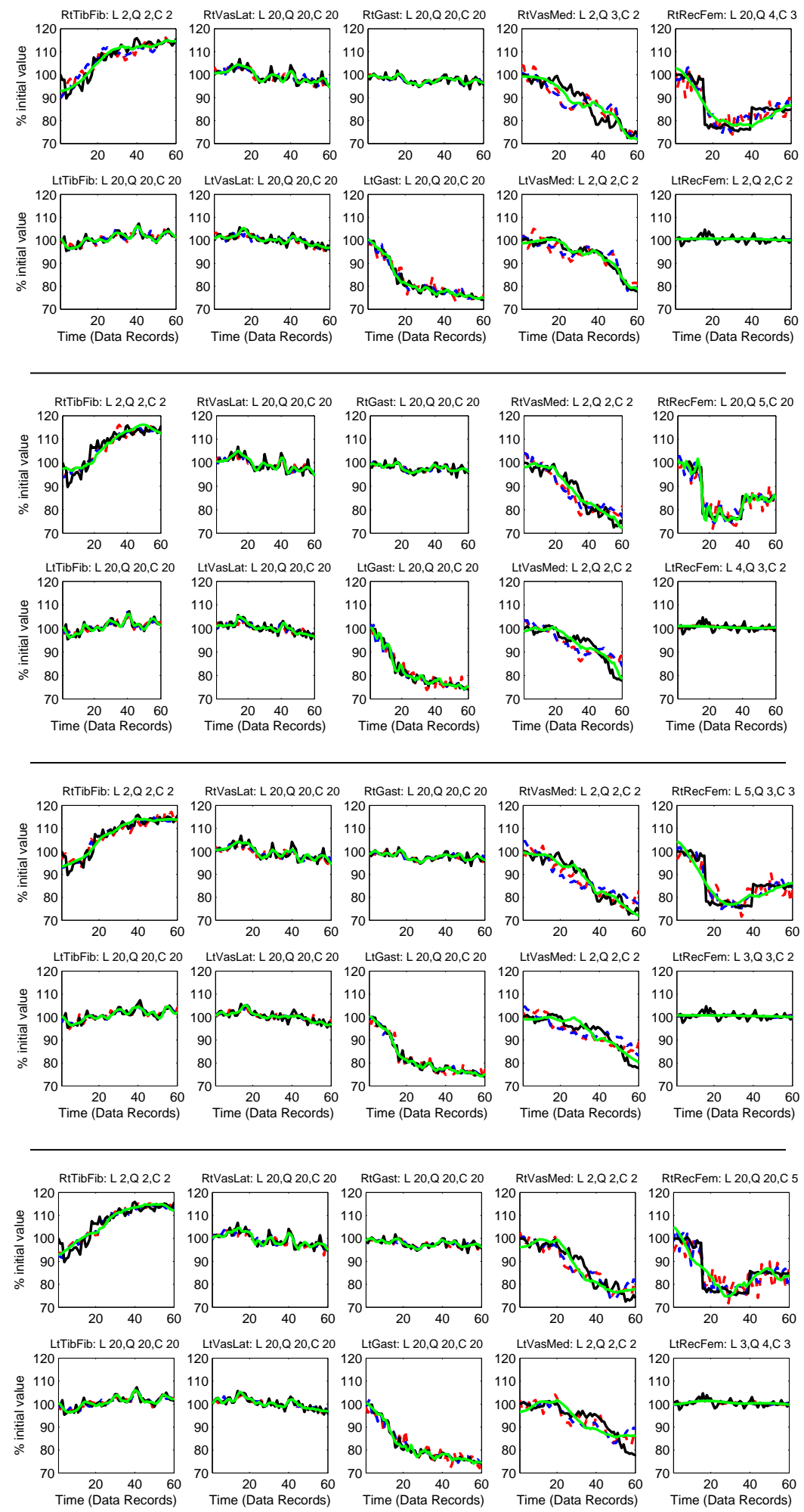

Figure D.3. Linear combinations of linear (red --), quadratic (blue --), and cubic (green -) SOCs projections onto MNF (black-) markers in a least squares sense for for subject 10: lean (top), hip (middle top), ankle (middle bottom), and knee (bottom) angles in the army walking experiment. 


\section{BIBLIOGRAPHY}

Southeastern univeristy and college coalition for engineering eduction, Lecture 19. Tissues, Organs, Protection, Support, and Movement, UNIT 4. ANIMAL SYSTEMS. [Online]. Available: http://www.succeed.ufl.edu/ content/abe2062/lect/lect_19/lect_19.htm

Department of Biology, Biosciences Web Site: www.nvo.com/jin, 1998-2010. [Online]. Available: http://www.nvo.com/jin/homepage1/

Alexandros, A., Otten, M., Nguyen, P., Hegger, R., and Stock, G., "Construction of the free energy landscape of biomolecules via dihedral angle principal component analysis," Journal of Chemical Physics, vol. 128, p. 245102, 2008.

Allen, D., Lannergren, J., and Westerbald, H., "Limits to human performance caused by muscle fatigue," Journal of Physiology and Experimental Physiology, pp. $7-10$.

Altis, A., Nguyen, H.Pand Hegger, R., and Stock, G., "Dihedral angle principle component analysis of molecular dynamics simulations," Journal of Chemical Physics, vol. 126, pp. 24 411-24 421, 2007.

Arihara, M. and Sakamoto, K., "Contribution of motor unit activity enhanced by acute fatigue to physiological tremor," Electromyogr Clin Neurophysiol, vol. 39, pp. 235-247, 1999.

Beck, T. W., Housh, T. J., Johnson, G. O., Weir, J. P., Cramer, J. T., Coburn, J. W., and Malek, M. H., "Comparison of fourier and wavelet transform procedures for examining the mechanomyographic and electromyographic frequency domain responses during fatiguing isokinetic muscle actions of the biceps brachii," Journal of Electromyography and Kinesiology, vol. 15, no. 2, pp. 190-199, 2005.

Berkooz, G., Holmes, P., and Lumley, Jonh, L., "The proper orthogonal decomposition in the analysis of turbulent flows," Annuals Review of Fluid Mechanics, vol. 25, pp. 539-575, 1993.

Bigland-Ritchie, B. and Woods, J. J., "Changes in muscle contractile properties and neural control during human muscular fatigue," Muscle $\& 3$ Nerve, vol. 11, pp. 251-279, 1984.

Brooks, G., Fahey, T., and White, T., Exercise physiology: human bioenergetics and its applications (2nd ed.). Mayfield Publishing Co. Mountain View, CA, 1996. 
Casdagli, M., Eubank, S., Farmer, J., and Gibson, J., "State space reconstruction in the presence of noise," Physica D: Nonlinear Phenomena, vol. 51, no. 1-3, pp. 52 - 98, 1991. [Online]. Available: http://www.sciencedirect.com/ science/article/B6TVK-46MV07J-7/2/fe34efc4f128e33716d7003ee4dd4d45

Chatterjee, A., "An introduction to the proper orthogonal decomposition," Current Science, vol. 78, no. 7, pp. 808-817, 2000.

Chelidze, D. and Cusumano, J. P., "Phase space warping: nonlinear time series analysis for slowly drifting systems," Philosophical Transactions of the Royal Society A, vol. 364, pp. 2495-2513, 2006.

Chelidze, D., Cusumano, J., and Chatterjee, A., "Dynamical systems approach to damage evalution tracking, part 1: desccription and experimental application," Journal of Vibration and Acoutics, vol. 124, no. 2, pp. 250-257, 2002.

Chelidze, D. and Liu, M., "Reconstructing slow-time dynamics from fast-time measurements," Philosophical Transaction of the Royal Society A, vol. 366, pp. 729-3087, 2008.

Chelidze, D. and Zhou, W., "Smooth orthogonal decomposition based modal analysis," Journal of Sound and Vibration, vol. 292, no. 3-5, pp. 461-473, 2006.

Chelidze, D., "Identifying multidimensional damage in a hierarchical dynamical system," Nonlinear Dynamics, vol. 31, pp. 307-322, 2004.

Chelidze, D. and Liu, M., "Dynamical systems approach to fatigue damage identification," Journal of Sound and Vibration, vol. 281, pp. 887-904, 2005.

Clancy, E. A., Farina, D., and Merletti, R., "Cross-comparison of time- and frequency-domain methods for monitoring the myoelectric signal during a cyclic, force-varying, fatiguing hand-grip task," Journal of Electromyography and Kinesiology, vol. 15, no. 3, pp. 256-265, 2005.

Ct, J. N., Mathieu, P. A., Levin, M. F., and Feldman, A. G., "Movement reorganization to compensate for fatigue during sawing," Experimental Brain Research, vol. 146, no. 3, pp. 394-398, 2002.

Cusumano, J., Chelidze, D., and Chatterjee, A., "Dynamical systems approach to damage evalution tracking, part 2: model-based validation and physical interpretation," Journal of Vibration and Acoutics, vol. 124, no. 2, pp. 258$264,2002$.

DeLuca, C. J., "Myoelectrical manifestations of localized muscular fatigue in humans," Critical Reviews in Biomedical Engineering, vol. 11, pp. 251-279, 1984.

DeLuca, C., "The use of surface electromyography in biomechanics," Journal of Applied Biomechanics, vol. 13, pp. 135-163, 1997. 
Dingwell, J. B., Napolitano, D. F., and Chelidze, D., "A nonlinear approach to tracking slow-time-scale changes in movement kinematics," Journal of Biomechanics, vol. 40, pp. 1629-1634, 2006.

Doebling, S., Farrar, C., Prime, M., and Shevitz, D., "Damage identification and health monitoring of structural and mechanical systems from changes in their vibration characteristics: A literature review." Tech. Rep. LA-13070-MS, Los Alamos National Laboratory, Los Almos, New Mexico 87545., Tech. Rep., 1996.

Ebaugh, D. D., McClure, P. W., and Karduna, A. R., "Effects of shoulder muscle fatigue caused by repetitive overhead activities on scapulothoracic and glenohumeral kinematics," Journal of Electromyography and Kinesiology, vol. 16, no. 3, pp. 224-235, 2006.

Encyclopedia, W. T. T., "Energy landscapes," http://en.wikipedia.org/wiki/Energy-landscape, Tech. Rep., 2010, date Accessed: October 2010.

Epstein, Y., Rosenblum, J., Burstein, R., and Sawka, M., "External load can alter the enery cost of prolonged exercise," Eur J Appl Physiol, vol. 57, pp. 243-247, 1988.

Farina, D., Fattorini, L., Felici, F., and Filligoi, G., "Nonlinear surface emg analysis to detect changes of motor unit conduction velocity and synchronization," Journal of Applied Physiology, vol. 93, pp. 1753-1763, 2002.

Farooq, U. and Feeny, B., "Smooth orthogonal decomposition for modal analysis of ramdomly excited systems," Journal of Sound and Vibration, vol. 316, pp. $137-146,2008$.

Febbraio, M. and Dancey, J., "Skeletal muscle energy metabolism during prolonged fatiguing exercise," J Appl Physiol, vol. 87, pp. 2341-2347, 1999.

Feeny, B. F. and Kappagantu, R., "On the physical interpretation of proper orthogonal modes in vibrations," Journal of Sound and Vibration, vol. 211(4), pp. 607-616, 1998.

Feeny, B., "On the proper orthogonal modes and normal modes of a continuous vibration system," Journal of Sound and Vibration, vol. 124(1), pp. 157-160, 2002.

Fraser, A. and Swinney, H., "Independent coordinates for strange attractors from mutual information," Physical Reviews A, vol. 33 (2), pp. 1134-1140, 1986.

Friedman, J., Bentley, J., and Finkel, R., "An algorithm for finding best matches in logarithmic expected time," ACM Transactions on Mathematical Sofware, vol. 3, pp. 209-226, 1977. 
Frykman, P., Harman, E., Knapik, J., and Han, K., "Backpack vs. front pack: Differential effects of fatigue on loaded walking posture (abstract only)," Med Sci Sports Exerc, vol. 26, p. 140, 1994.

Gandevia, S. C., "Spinal and supraspinal factors in human muscle fatigue," Physiological Reviews, vol. 81, pp. 1725-1789, 2001.

Gates, D. H., "The role of muscle fatigue on movement timing and stability during repetitive tasks," Ph.D. dissertation, University of Texas at Austin, 2009.

Gates, D. and Dingwell, J., "Effects of muscle fatigue on variability and temporal correlations in movement timing errors," Experimental Brain Research, vol. 187, pp. 573-585, 2008.

Geddes, L., "Superhuman," New Scientist, vol. 07-28, pp. 35-41, 2007.

Güttler, S., Kantz, H., and Olbrich, E., "Reconstruction of the parameter spaces of dynamical systems," The American Physical Society, vol. 63, p. 056215, 2001.

Heller, M., Sukopp, M., Tsomaia, N., John, M., Mierke, D.Fand Reif, B., and Kessler, H., "The conformation of cyclo(-d-pro-ala4-) as a model for cyclic pentapeptides of the dl4 type," Journal of the American Chemical Society, vol. 128 , pp. $13806-13814,2006$.

Hingtgen, B., McGuire, J., Wang, M., and Harris, G., "An upper extremity kinematic model for evaluation of hemiparetic stroke," Journal of Biomechanics, vol. 39, pp. 681-688, 2006.

Humphrey, W., Dalke, A. ., and Schulten, K., "Vmd - visual molecular dynamics," Journal of Molecular Graphics, vol. 14, pp. 33-38, 1996.

Hunter, S. K., Critchlow, A., Shin, I.-S., and Enoka, R. M., "Fatigability of the elbow flexor muscles for a sustained submaximal contraction is similar in men and women matched for strength," Journal of Applied Physiology, vol. 96, pp. 195-202, 2004.

Jorgensen, W. L., Maxwell, D. S., and Tirado-Rives, J., "Development and testing of opls all atom force field on conformational energetics and properties of organic liquids," Journal of the American Chemical Society, vol. 118, pp. 11 225-11 236, 1996.

Jorgensen, W. L. and Tirado-Rives, J., "The opls potential functions for proteins. energy minimizations for crystals of cyclic peptides and crambin," Journal of the American Chemical Society, vol. 110(6), pp. 1657-1666, 1998.

Kantz, H. and Schreiber, T., Nonlinear Time Series Analysis. Cambridge University Press, 2004. 
Kennel, M., Brown, R., and Abarbanel, H., "Determining embedding dimension for phase-space reconstruction using a geometrical construction," Physical Reviews A, vol. 45 (6), pp. 3403-3411, 1992.

Kershen, G., F, P., and Golinval, J., "Physical interpretation of independent component analysis in structural dynamics," Mechanical Systems and Signal Processing, vol. 21, pp. 1567-1575, 2007.

Knapik, J., Harman, E., and Reynolds, K., "Load carriage using packs: A review of physiological, biomechanical and medical aspects," Appl Ergon, vol. 27, pp. 207-216, 1996.

Kolinski, A. and Skolnick, J., "Reduced models of proteins and their applications," Polymer, vol. 45, pp. 511-524, 2004.

Lange, F. and Grubmuller, H., "Can principle components yield a dimension reduction desccription of protein dynamics on long time scales," Journal of Chemical Physics B, vol. 110, pp. 22 842-22852, 2006.

Lange, F. and Grubmuller, H., "Collective langevin dynamics of conformational motions in proteins," Journal of Chemical Physics, vol. 124, pp. 214903$214921,2006$.

Leao, R, N. and Burne, J, A., "Continuous wavelet transform in the evaluation of stretch reflex responses from surface emg," Journal of Neuroscience Methods, vol. 133, pp. 115-125, 2004.

Liang, Y. C., Lee, H. P., Lim, S. P., Lin, W. Z., Lee, K. H., and Wu, C. G., "Proper orthogonal decomposition and its applications-part i: Theory," Journal of Sound and Vibration, vol. 252, pp. 527-544, 2002.

Lu, M., Poon, B., and Ma, J., "A new method for course-grained elastic normal mode analysis," Journal of Chemical Theory and Computation, vol. 2, pp. 464-471, 2006.

MacIsaac, D., Parker, P., and Scott, R., "The short-time fourier transform and muscle fatigue assessment in dynamic contractions," Journal of Electromyography and Kinesiology, vol. 11, no. 6, pp. 439-449, 2001.

Madigan, M. L. and Pidcoe, P. E., "Changes in landing biomechanics during a fatiguing landing activity," Journal of Electromyography and Kinesiology, vol. 13, no. 5, pp. 491-198, 2003.

Maisuradze, G., Liwo, A., and Scheraga, H., "Principle component analysis for protein folding dynamics," Journal of Molecular Biology, vol. 385, pp. 312329, 2009. 
Martini, F. H., Timmons, M. J., and Tallitsch, R. B., Human Anatomy. PrenticeHall Inc., 1995.

Mazur, A. K., "Quasi-hamiltonian equations of motion for internal coordinate molecular dynamics of polymers," Journal of Computational Chemistry, vol. 18(11), pp. 1354-1364, 1997.

Mazur, A. K., "Hierarchy of fast motions in protein dynamics," Journal of Physical Chemistry B, vol. 102, pp. 473-479, 1998.

Mizrahi, J., Verbitsky, O., Isakov, E., and Daily, D., "Effect of fatigue on leg kinematics and impact acceleration in long distance running," Human Movement Science, vol. 19, no. 2, pp. 139-151, 2000.

Moon, F. and Holmes, P., "A magnetoelastic strange attractor," Journal of Sound and Vibration, vol. 65, pp. 275-296, 1979.

Mu, Y., Nguyen, H., and Stock, G., "Energy landscape of a small peptide revealed by dihedral angle principle component analysis," Proteins: Structure, Function, and Bioinformatics, vol. 64, pp. 795-797, 2006.

Oka, H., "Estimation of muscle fatigue by using emg and muscle stiffness," in 18th Annual International Conference of the IEEE Engineering in Medicine and Biology Society, Amsterdam 1996, 1996.

Patton, J., Kaszuba, J., Mell, R., and Reynolds, K., "Physiological responses to prolonged treadmill walking with external loads," Eur J Appl Physiol, vol. 63, pp. 89-93, 1991.

Perotto, M, A. and Delagi, F, E., Anatomical guide for the electromyographer: the limbs and trunk. Charles C Thomas, 2005.

Petrone, P. and Pande, V., "Can conformational change be described by only a few normal modes?" Biophysical Journal of Medicinal Chemistry, vol. 90, pp. 1583-1593, 2006.

Rao, S., Bontruger, E., Gronley, J., Newsam, C., and Perry, J., "Three-dimensional kinematics of wheelchair propulsion," IEEE Transactions on Rehabilitation Engineering, vol. 4, no. 3, pp. 152-160, 1996.

Sadeghi, H., Allard, P., Prince, F., and Labelle, H., "Symmetry and limb dominance in able-bodied gait: a review," Gait and Posture, vol. 12, pp. 34-45, 2000 .

Sauer, T., Yorke, J. A., and Casdagli, M., "Embedology," J. Stat. Phys., vol. 65, no. 3-4, pp. 579-616, 1991. 
Schmidt, R., Disselhorst-Klug, C., Silny, J., and Rau, G., "A marker-based measurement procedure for unconstrained wrist and elbow motions," J Biomech, vol. 32, pp. 615-621, 1999.

Selen, L. P. J., Beek, P. J., and van Dien, J. H., "Fatigue-induced changes of impedence and performance in target tracking," Experimental Brain Research, vol. 181, pp. 99-108, 2007.

Shaw, S. and Pierre, C., "Non-linear normal modes and invariant manifolds," Journal of Sound and Vibration, vol. 150(1), pp. 170-173, 1991.

Shaw, S. and Pierre, C., "Normal modes for non-linear vibratory systems," Journal of Sound and Vibration, vol. 164(1), pp. 85-124, 1993.

Shaw, S. and Pierre, C., "Normal modes of vibration for non-linear continuous systems," Journal of Sound and Vibration, vol. 169(3), pp. 319-347, 1994.

Shlens, J., "A tutorial on principal component analysis," Institute for nonlinear science, University of California, San Diego, La Jolla, CA 92093, Tech. Rep., 2005.

Skjaervan, L., Hollup, S., and Reuter, N., "Normal mode analysis for proteins," Journal of Molecular Structure: THEOCHEM, vol. 898, pp. 42-48, 2009.

Song, M., Segala, D. B., Dingwell, J. B., and Chelidze, D., "Slow-time changes in human muscle fatigue are fully represented in movement kinematics," Journal of Biomechanical Engineering, vol. 131, pp. $021004(1-11), 2009$.

Sparto, P., Parnianpour, M., Reinsel, T., and Simon, S., "The effect of fatigue on multijoint kinematics, coordination, and postural stability during a repetitive lifting test," Journal of Orthopaedic \& Sports Physical Therapy, vol. 25, no. 1, pp. 3-12, 1997.

Tang, D., Li, A., Attar, P., and Dowell, E. H., "Reduced order dynamic model for polysaccharides molecule attached to an atomic force microscope," Journal of Computational Physics, vol. 201, pp. 723-752, 2004.

Torvik, V., Triantaphyllow, E., Liao, T., and Waly, S., "Predicting muscle fatigue via electromyography: A comparative study," in Proceedings of the 25th International Conference on Computers and Industrial Engineering, pages 277-280, 1999.

Vakakis, A., "Non-linear normal modes (nnms) and their application in vibration theory: An overview," Mechanical Systems and Signal Processing, vol. 11(1), pp. 3-22, 1997. 
Veldpaus, F., Woltrin, H., and Dortmans, L., "A least-squares algorithm for the equiform transformation from spatial marker co-ordinates," Journal of Biomechanics, vol. 21, pp. 45-54, 1998.

Verdes, P., Granitto, Pand Navone, H., and Ceccatto, H., "Nonstationary timeseries analysis: accurate reconstruction of driving forces," Phys. Rev. Lett, vol. 87(12), p. 124101(4), 2001.

Verdes, P., Granitto, P., and Ceccatto, H., "Overembedding method for modeling nonstationary systems," Phys. Rev. Lett, vol. 96(11), p. 118701(4), 2006.

Voge, K. and Dingwell, J., "Relative timing of changes in muscle fatigue and movement coordination during a repetitive one-hand lifting task," in Proceedings of the 25th Annual International Conference of the IEEE Engineering in Medicine and Biology Society. Cancun, Mexico: Institute of Electrical and Electronics Engineers, 2003, pp. 1807-1810.

Volkwein, S., "Model reduction using proper orthogonal decomposition. lecture notes,", Institute of Mathematics and Scientific Computing, University of Graz, Tech. Rep., 2008.

Winter, D. A., Biomechanics and motor control of human movement. John Wiley \& Sons. Inc., 2005.

Wu, G., van der Helm, F., Veeger, HE amd Makhsous, M., Van Roy, P., Anglin, C., Nagels, J., Karduna, A., McQuade, K., Wang, X., Werner, F., and B, B., "Isb recommendation on definitions of joint coordinate systems of various joints for the reporting of human joint motion-part ii: shoulder, elbow, wrist and hand," Journal of Biomechanics, vol. 38, pp. 981-992, 2005.

Yang, L., Eyal, E., Bahar, I., and Kitao, A., "Principal component analysis of native ensembles of biomolecular structures (pca nest): insights into functional dynamics," Bioinformatics (Oxford, England), vol. 25, pp. 606-614, 2009.

Zhang, X., Nikiforovich, G., and G.R, M., "Conformational templates for rational drug design: Flexibility of cyclo(d-pro1-ala2-ala3-ala4-ala5) in dmso solution," Journal of Medicinal Chemistry, vol. 50, pp. 2921-2925, 2007.

Zhou, W. and Chelidze, D., "Blind source seperation based vibration mode identification," Mechanical Systems and Signal Processing, vol. 21, pp. 3072-3087, 2007. 